\title{
Lepidopterorum Calaloglus
}

editus $a b$

\section{Embrik Strand}

\section{Pars 51:}

\section{H. Stichel}

\section{Brassolidae.}
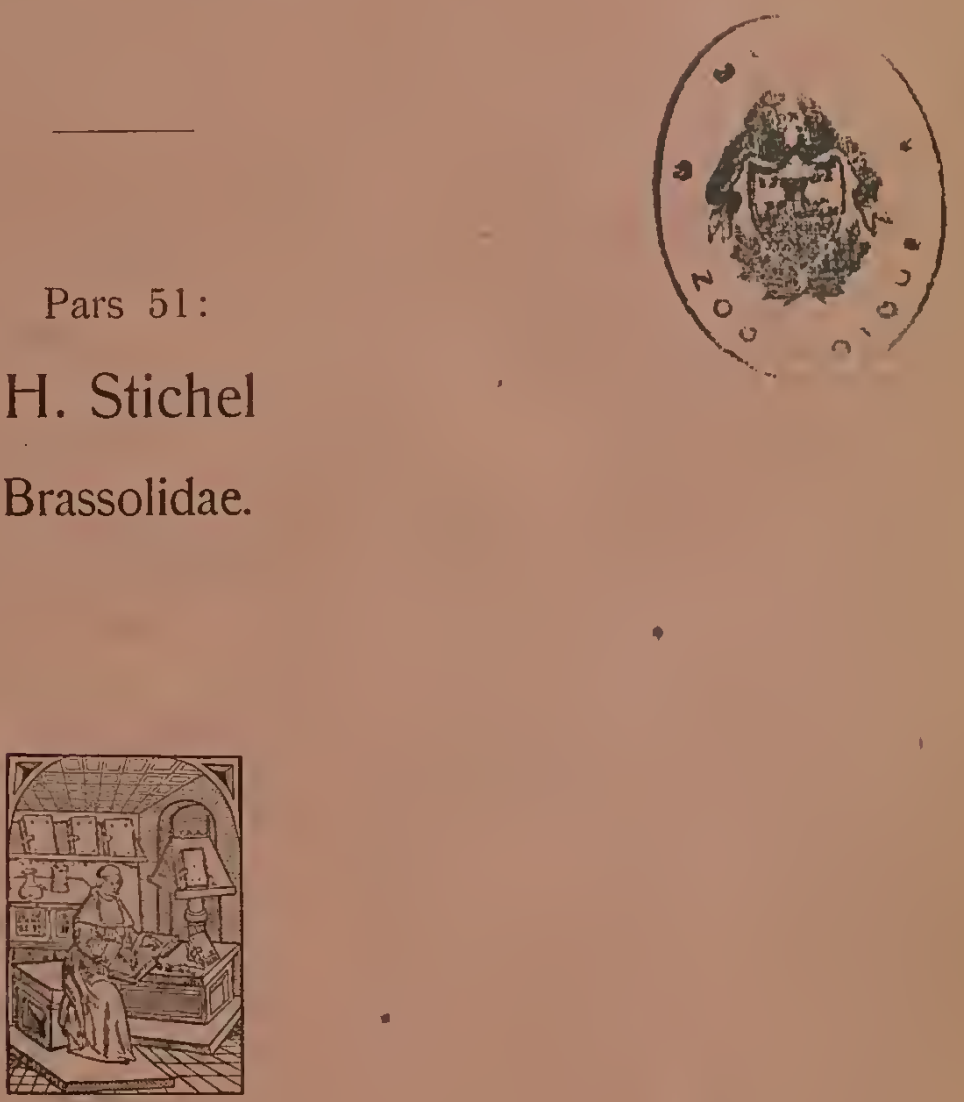

\section{W. Junk}

Berlin W. 15

27. IV. 1932.

Beilage: Entgegnung der Herren Gaede und Seitz auf die von Hern Kern y veröffentlichte Kritik der Partes $\$ 3,46$ und 48 des "L. C.". 


\section{Lepidopterorum Catalogus}

Pars 1: Ch. Iurivillius, Chrysopolomidae. 1911. 4 p.

2: А. Parenstecher, Callidulidae. 1911. 14 p.

3: A. Pagenstedier, libytheilae. 1911. $12 \mathrm{p}$.

t: II. Wagner et R. P'fitzuer, Hepialidac. 1911. 26 p.

5: F. Strand, Toetuidae: Araristinae. 1912. 82 p.

6: E. Mryrick, Adelidae, Micropterygidae, Gracilariadae. 1912. 68 p.

7: II. Zerny, Srntomilat. 1912. $159 \mathrm{p}$.

8: L. B. P'ront, Geometridae: Brephinac, Ocnochrominae. 1912. 94 p.

9: P. Mabille, Hesperilae: *ubf. Pyrhopprinae. - MeDunnongh, Megathrmidac. 1912. $22 \mathrm{p}$.

10: H. Meyrick, Tortricidae. 1912. $86 \mathrm{p}$.

11: II. El1ringlian et K. Jordan, Nymphalidae: Subfin. Acraeinae. 1913. $65 \mathrm{p}$.

12, 18, 21, 23: II. Wagner, Sphingidae. 1913-1919. 420 p.

13: E. Heyrick, Caposindae, ILediodinidae, Glyplipterygidac. 1913. $53 \mathrm{p}$.

14: L. B. Pront, Geometridae Sulfam. Jenitheinae. 1913. $192 \mathrm{p}$.

15: K. W. von Dalla Torre, Castuiidac: Subfam. Castninae, Teocastniinae. Penphigostolinae. 1913. 28 p.

16: E. Strand, Brahmacidae. - 11. G. Dyar et E. Strand: Mlegalopsgidne, 1)alceridae, Epipyropidae. 1913. $35 \mathrm{p}$.

17: E. Ieyriek, Pterophoridae, Orneodidac. 1913. 44 p.

19: E. Ieyrick, Hrponomentidae, Pnntelidac, Amplitheridae. 1914. $64 \mathrm{p}$.

20: lí. W. von Dalla Torre. Thyrididac. 1914. $55 \mathrm{p}$.

22, 24, 26: E. Straud Arotidae, 1919-1922. 900 p.

25: K. W. vou Dalla Torre, Crmatophoridae. 1921. 38 p.

27: F. Bryk, Baroniidae, Teinopalpidar, Parnassiiclac. 1923. 247 p.

28: K. W. ron Ialla 'T'ore et L. Strand, Lepidarbelidae. - E. Strand, ITeterogrnididae. 1923. $14 \mathrm{p}$.

29: K. W. von Malla Torre, Cossidac, 1923. 63 p.

30: K. W. von Dalla Torre, Epiplemidac, 【raniidae. 1924. 57 p.

31: K. W. rou Jalla Torre et E. Straud, Aegeriidae. 1925. $282 \mathrm{p}$.

32: R. ran Eectie, Cochlidionidae (Limacodidae). 1925. 81 p.

33: H. Burgeti, /ygnenidae 1: Generis Zygaena palnearctica pars. 1926. $91 \mathrm{p}$

34: K. W. von Dalla Torre et E. Straud, Pșrchidac. 1929. 215 p.

Fortsetzung siche 4. Umschlagseite. 


\section{Lenidlopteropum Calaloglus \\ editus $a b$}

Embrik Strand

Pars 51:

\section{H. Stichel}

Brassolidae.
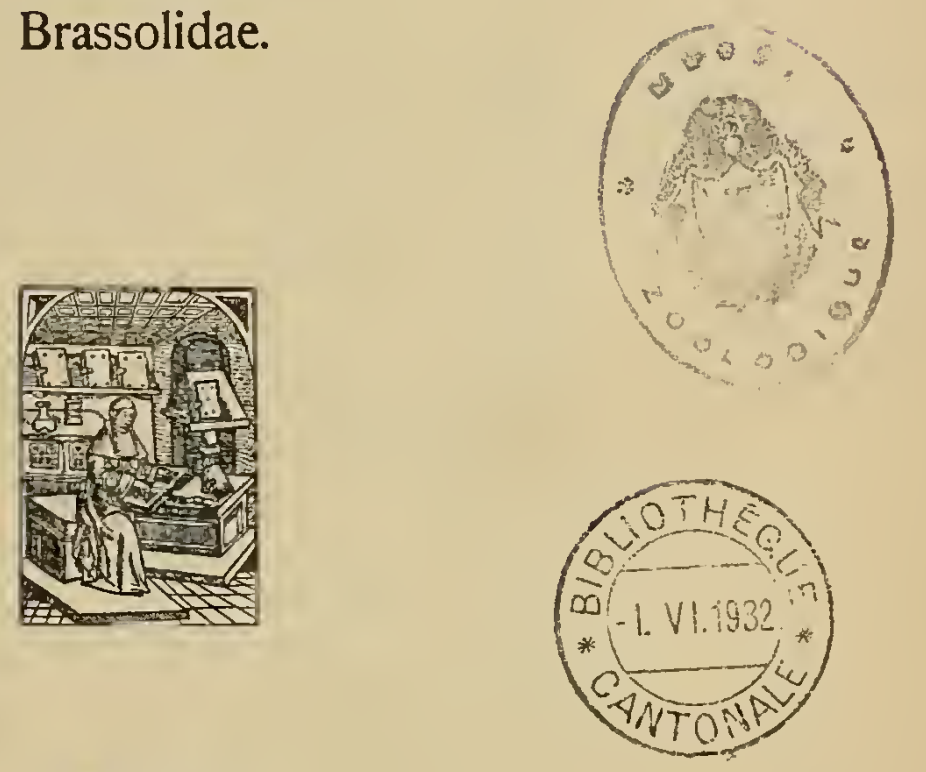

W. Junk

Berlin W. 15 1932. 



\title{
Familia Brassolidae ${ }^{\text {( })}$
}

\author{
Auct. H. Stichel
}

„Brassolides", Boisduval, Spéc. Géner. Lépid., v. 1, p. 166 (1836). - Id., Cuvier, Règne Animal., ed. 3, v. 3, p. 232 (1836). — ,Brassolites" v. 3, p. 453, 454 (1840). - "Brassolides", J. O. Westwood (sec. Boisduval), Introd. Classif. Ins., v. 2, p. 345 (1840). - „Brassolites", D[oyère] (sec. Blanchard), Orbigny, Dict. Hist. Nat., v. 2, p. 728 (1842). - Brassolidae, E. Doubleday, List Lep. Brit. Mus., v. 1, p. 117 (1844). - "Brassolites", E. Blanchard, Hist. Ins., v. 2, p. 334, 340 (1845). - Brassolidae + Morphidae (part.), (E. Doubleday \&) J. O. Westrood, Gen. Diurn. Lep., v. 2, p. 350,332 (1851). - Brassolitae + Morphitae (part.) (Divisiones), Ohenu \& H. Lucas, Enc. Hist. Nat. Papill., p. 172, 164 (1851-53). Brassolidac, Wallace, Trans. Ent. Soc. London, n. ser., v. 2, p. 261 (1853). - B. + Morphidae (part.), Ménétriés, Enum. Corp. An. Ac. Petropol., Lep., par's 1, p. 39, 37 (1855). - Brassolinae (Subfam.), II. W. Bates, Journ. Entom., v. 1, p. 220, v. 2, p. 176 (1861 \& 61). - Brassolina, Herrich-Schäffer, Corr.-Bl. Zool-Min. Ver. Regensb., v. 18, p. 91, 98; v. 19, p. 64 (1864 \& 65). - Brassolinae (Subfam.), A. G. Butler, Cat. Diurn. Lep. Fabr., p. 39 (1869). - Id., Lep. Exot., p. 29, 125 (part.) (1870 \& 73). - W. F. Kirby, Cat. Diurn. Lep., p.125; Suppl., p. 716, 847 (1871 \& 77). H. W. Bates, Cist. Ent., v. 1, p. 73 (1872). - A. G. Butler, Trans. Ent. Soc. London, p. 425 (1874). - "Brassolides" + "Pavonides", Capronnier, Ann. Soc. Ent. Belg., v. 17, p. 28 (1874). - Brassolinae (Subfam.), Herb. Druce, Proc. Zool. Soc. London, p. 217 (1876). - Brassolidae, Wallace, Geogr. Distrib. Anim., v. 2, p. 13, 90, 472 (1876). - „Brassolinen", C. Crüger, Verh. Ver. Naturw. Unterh. Hamburg, v. 2 (1875), p. 129 (1876). - Brassolinae, A. G. Butler, Trans. Ent. Soc. London, p. 113 (1877). W. F. Kirby, Entomologist, v. 10, p. 199, 201 (1877). — „Brassolinen", Fr. Müller, Jenaische Zeit. Naturw., v. 11, p. 102 (1877). - "Brassoliden", „Brassolinen", Td., Kosmos, Leipzig, v. 1, p. 391; 394 (1877). - Brassolinae, Brassolidae, IV. F. Kirby, Entomologist, v. 11, p. 25, 26 (1878). - Brassolidae, H. Burmeister, Descr. Phys. Rép. Argent., v. 5, p. 193 (1878). - "Brassolides" ou „Pavonindes", Bar, Ann. Soc. Ent. France, ser. 5, v. 8, p. 12, 13, 20 (1878). - Brassolinae, F. Müller, Trans. Int. Soc. London, p. 214 (1878). - "Brassolimen", C. Crüger, Verh. Ver. Naturw. Unterh. Hamburg, v. 4 (1877), p. 193 (1879). - Brassolidae, H.: G. Jäger, Handwörterb. Zool., v. 1, p. 501 (1880). P. H. Gosse, Entomologist, v. 13, p. 201 (1880). - Brassolinae, Swinton, Ins. Var., p. 73 (not. morphol. sec. F. Müller) (1880). - B. (Subfam.), F. D. Godman \& O. Salvin, Trans. Ent. Soc. London, p. 122 (1880). - Tid., Biol. Centr.-Amer., Rhop., V.o 1, p. 122 (1881). - "Brassolides" + ,Pavonides" (Tribus), Capronnier, Ann. Soc. Ent. Belgd, v. 25, p. 101 (1881). - Brasso-

*) Die erreichbare Literatur ist bis 1930 einschließlich berücksichtigt. Lepidopterorum Catalogus 51. 
lidae, J. (sec. W. F. Kirby): G. Jäger, Handwörterb., Zool., v. 2, p. 403 (1883). - „Brassoliden", E. Gerhard, Berlin. Ent. Zeit., v. 27, p. 179 (distrib. geogr.) (1883). - Brassolinae, G. W. Müller, Zool. Jahrb. System., v. 1, p. 593, 605, 616, 633 (1886). L. Glaser, Cat. Etymol. Col. Lep., p. 278 etc. (1887). - Brassolidae, Knauer, Handwörterb. Zool., p. 124 (1887). - "Brassoliden", O. Staudinger (\& Schatz), Exot. Schmett., v. 1, p. 211 (1887). Röber, ibid., v. 2, p. 190 (1889). - Richelmann, Tagebl. Deutsch. Naturf. Versamml., 61, p. 68 (1889). - Brassolidae, A. Seitz, Zool. Jahrb. System., v. 4, p. 918 (not. biol.) (1859). - Id., Ent. Zeit. Stettin, v. 51, p. 29 (not. biol.) (1890). - Brassolinae, E. Haase, Deutsch. Lint. Zeit. Iep. (Tris), v. 4, p. 33 (1891). - F. D. Godman \& O. Salvin: Whymper, Travels Great Andes, Suppl. Append., Extr. p. 99 (1892). - B. (Subfam.), W. F. Kirby, Handb. Lep., v. 1, p. 199 (1894). - Chapman, Ent. Rec. Journ. Tar., v. 6, p. 128 (1895). - „Brassoliden", O. Michaol, Deutsch. Ent. Zeit. Lop. (Iris), v. 7 (1594), p. 232 (1895). - Weymer, Ent. Zeit. Stettin, v. 55, p. 322 (1895). - Brassolidae, Pavonidae (Gruppe oder Subfam.), Bönninghausen, Verh. Nat. Ver. Hamburg, v. 9, (1894-95), p. 25, 40, p. 37 (1896). - "Brassolinlen", Brassolinae (Subfam.), Brassolina (Stirps), E. Reuter, Acta Soc. Sci. Fenn., v. 22 , p. 111, 553 (1896). - Brassolinae, id., ibid., p. 553 (1896). - Brassolinae (Subfam.), 'I'ristan, Ins. Costa Rica, p. 18 (1897). - K. Jordan, Nov. Zool., v. 5, p. 359 (1898). - E. Reuter, Ent'. Rec. Journ. Var., v. 10, p. 76, 96 (1898). - T. Ai Chapman, 1. of v. 11, p. 87 (1899). - K. Jordan, Ent. Nachr., v. 26, p. 272 (Recens. crit. Syst. Lep. Hildesiae Grotei, 1895) (1900). - W. F. Kirby: Jac. Hübnor \& Geyer, Samml. Exot. Schmett, Neue Ausg., v. 3, p. 50 (1901). - Brassolidae, Therese v. Bayern, Berlin. Ent. Zeit., v. 46, p. 265 (1901). - "Brassolides", Henneguy, Ins., p. 194 (not. morphol.) (1904). - Brassolinae (Subfam.), H. Stichel, Gen. Ins., Fase. 20, p. 1 (1901). - W. J. Kaye, Trans. Ent. Soc. London, p. 165 (1904). - Brassolidae, Silva, Relat. Contrib. Hist. Nat. Lep. Brasil (Congr. Scient. Lat.-Amer., 1905, v. 3 B), p. 87

(1907). - Brassolinae, Fruhstorfer, Ent. Zeit. Stettin, v. 68, p. 128 (1907). - „Brassoliden", id., Int. Ent. Zeit., v. 1, p. 29 (1907) H. Stichel (sec. Röber), Soc. Ent., v. 22, y. 92 (1907). - Brassolidac id., Tierreich, Lief. 25, p. 1 (1909). - "Brassoliden", A. Pagenstecher, Geogr. Verbr. Schmetterl., p. 413 (1909). - Fassl, Soc. Entom., v. 24, p. 116; v. 27, p. 54 (ova) (1909 \& 12). Brassolidae, id., Fauna Exot., v. 1, p. 26 (distrib. vertical.) (1911). - Fruhstorfer: A. Seitz, GroBsehmett. Erde, v. 5, p. 285 (1912). - Brassotininae + Caligoninae (Subgen.), id., 1. c., p. 286; p. 290 (1912). - Brassolinae (Subgen.), H. G. Dyar, Proc. Un. St. Nat. Mus. Washington, v. 45, p. 636 (1913). - Id., 1. cu, จ. 47 , p. 144 (1914). - Hild. Schultz, Deutsch. Ent. Zeit., p. 26 (1914). - W. J. Kaye, Trans. Ent. Soc. London, p. 547 (1914). "Brassoliden", Fassl, Ent. Rundschau, v. 31, p. 37, 44 (1914). - R. (\& H.) Heymons, Brehms Tierleben, ed. 4, v. 2, p. 291 (1915). - Brassolididae, Strand, Lep. Niepelt., v. 2, p. 12, 27 (1916). - Brassolidae + Caligonidae, Rothschild, Nov. Zool., v. 23, p. 309, 314; p. 309 (1916). - B., Fussl, Ent. Rundschau, v. 33, p. 26 (1916). - Id., l. c., v. 35, p. 31 (1918). - Strand, Soc. Ent., v. 33, p. 19 (1918). - Brassolinae, Ilampson, Novit. Zool., v. 25, p. 385 (1918). - Brassolidae, Zikán, Biol. Beitr. (Ihering Festschr. Deutsch. Ver. Wissensch. Kunst, Sao Paulo, v. 1), p. 150 (1920). - G. Lederer, Handb. prakt. Entom., v. 2, p. 61 (not. biol. sec. A. Seitz) (1921). - Ealand, Ins. Life, p. 145 (1921). Brassolinae (Subfam.), Campos, Revist. Co. Nacion. Vic. Rocafuerte, 
Nr. 4, p. 30 (1921). - Brassolidae, Ferreira d'Almeida, Mél. Lépid., p. V (1922). - Jurriaanse, Tijdschr. Ent., v. 26, p. 147 (1923). P. Köhler, Fauna Argent. (Zeit. wiss. Ins.-Biol., v. 18, Beilage); v. 1, p. 21 (1923). - Giacomelli, Revist. Chil. Hist. Nat., v. 27, p. 16 (1924). - Schwanwitsch, Proc. Zool. Soc. London, p. 520, 522 (Morphol.) (1924). - M. Hering \& W. Hopp, Deutsch. Ent. Zeit. Iris, v. 39, p. 193 (1925). - II. Stichol, Nouo Beitr. syst. Ins.kunde, v. 3, p. 58 (1925). - Brassolini, Handlirsch: Chr. Schröder, Handb. Entom., v. 3. p. 939 (1925). - Brassolidae, Strand, Bull. Soc. Zool. France, v. 51, p. 398 (1926). - Eltringham, Trans. Ent. Soc. London, p. 367 (1926). - ,Brassolinen", Holdhaus: Chr. Schröder, Handb. Entom., v. 2, p. 731 (1927). - Brassolidae, W. J. Holland, Encyol. Amer., v. 17, p. 308 (1928). - Talbot, Bull. Hill Mus., v. 2, p. 199 (1929). - A. Seitz (sec. E. Haase), Ent. Rundschau, v. 45, p. 3 (1928). - Zikan, l. c., v. 45, p. 10 (1928). - Thering, Entom. Rundschau, v. 46, p. 41 (1929), - F. Hoffmann, Zoit. wiss. Insektbiol., v. 25, p. 94 (1930). - Brassolinae (part.), O. B. Williams, Migrat. Butterfl., p. 241 (1930).

Equites Achivi (part.), p. 458, $461+$ Danai festivi (part.), p. 458, 470, Linné, Syst. Nat., ed. 10 (1758). Equites (part.), Gronov (Gronovius), Zoophyl., p. 187 (1764). - E. Adrivi (part.) + Danai Festivi (part.), Linné, Mus. Ludov. Ulr., p. 181, 200; 251 (1764). - Linné-Houttyn, Natuurl. Hist., v. 1. XI, p. 186 (1766-69). - Linné, Syst. Nat., ed. 12 (13), p. 748 ; 765 (1767). - P. L. S. Müller, Naturs. Linné, v. 5. I, p. 571; 595 (1774). J. C. Fabricius, Syst. Ent., p. $449 ; 479$ (1775). - E. A. (part.) + D. $H^{r}$. (part.) + Nymphales Gemmati (part.), Groeze, Ent. Beytr., v. 3, I, p. 46; 186; 227; 286 (1779). - E. Achiui (part.) + Danai festiui (part.), J. C. Fabricius, Spec. Ins, v. 2, p. 10; 52 (1781). - E. Archiui (part.) +. D. f. (part.) + Nymphales gemmati (part.), id., Mant. Ins., v. 2, p. 25; 34 (1787). - E. Achiui (part.) -.- Consules (part.), (Jablonsky \&) Herbst, Naturs. Ins., Schmetterl., v. 3 , p. 5 ; .6 , p. $5(1788$ \& 93). - E. Achivi (part.), p. $2234+$ Danai festivi (part.), p. 2273 + Nymphales Gemmati (part.), p. 2290, Gmelin-Linné, Syst. Nat., ed. 13, v. 1. V $(1790)$ - - E. A. (part.), Al G. Olivier, Aot. Soc. Hist. Nat. Paris, v. 1, p. 121 (1792). Eestivi (part.) † Nymphales (part.), J. O. Fabricius, Syst. Ent., v. 3. I, p. 39;61 (1793). - Papiliones E. Achivi (part.), Esper, Ausl. Schmett., p. 161 (1801). - Festivi (part.) + Nymphales (part.), Turton, Gen. Syst. Nat. Linné, v. 3, II, p. 58, 77 (1806). - Danai F. (part.), G. Shaw, Gen. Zool., v. 6. I, p. 212 (1806).

Equites Troes (part.), J. Roemer, Gen. Ins. Linné, Fabr., p. 67 (1789).

Nymphalis Aemmati (part.), C. Stoll, Essai Syst. Lep. (P. Oramer, Pap. Exot., v. 4, Append.), p. 7 (1782). - Nymphales (part.), Fr. Weber, Nomencl. Intom., p. 100 (1795). - N. proprie dicti (part.), Latreille, Hist. Crust. Ins., v. 3, p. 592 (1802). - Nymphales (part.), Gravenhorst, Vergl. Ubersicht Zool. Syst., p. 312 (1807).

$N$ ymphalidae (part.), Swainson (sec. Latrcille), Philos. Mag., ser. 2, v. 1, p. 187 (1827). - J. O. Westwood, Introd. Classif. Ins., v. 2, p. 353 (1840). - „Nymphalides", "Nymphaliens", (Tribus), L[ucas]: Orbigny, Dict. Hist. Nat., v. 9, p. 515; Atl., v. 2, p. 17 (1847 \& 49). - 
"Nymphaliens" (part.), Fauvel, Bull. Soc. Linn. Normandie, v. 6 (1860-61), p. 135 (1862). - Nymphalinae (part.), Nymphalidae (part.), J. G. Wood, Insects abroad, p. 592, 617 (1874). - Nymphalidue (part.), O. Orüger (sec. Hewitson), Berlin. Ent. Zeit., v. 25, p. 109 (1881). - J. H. Comstock, Erol. Taxon. Class. Ins., p. 99, 112 (1893). J. H. \& A. B. Comstock, Manual Stud. Ins., p. 207 (1895). - "Nymphaliden", Nymphalidae (part.), F. Karsch, Ent. Nachr., v. 24, p. 298, 301 (1898). - Nymphalidae (part.), Lefroy, Syn. Olass. Ins., p. 12 (1913). - Nymphalididae (part.), Strand, Lep. Nicpeltian., v. 1, p. 29, 62 (1914). - Nymphalinae (Subfam.) (part.), Handlirsch: Chr. Schröder, Handb. Entom., v. 3, p. 938 (1925). - Nymphalidae (part.), Iloldhaus, ibid., v. 2, p. 730 (1927).

Potamides $S$ u perbae (part.) + P. Conspicuae (part.), Jac. Hübner \& Geyer, Samml. Exot. Schmett., v. 1-3, In$\operatorname{dex}(1806-41)$.

Pa pilionides (Fam.) (part.), Latreille, Gen. Crust. Ins., v. 4, p. 193 (1806-09). - Id., Consid. Gén., p. $343(1810)$. - Papiliones Nymphales (part.), Jac. Hübner, Cat. Coll. Franck, p. 75 (1826). - Papilionides (Natio) (part.), Billberg, Enum. Ins., p. 75 (1820). $-P$. (Sectio) (part.), Verloren, Cat. Lep. Ins. Cramer, v. 2, p. 173 (1837). - P. (part.), S. H. Scudder, Trans. Amer. Ent. Soc., v. 6, p. 69, 72 (1877). - Papilionina (part.), Bodine, Trans. Amer. Ent. Soc., v. 23, p. 42 (1896).

Conspicuae (part.), Jac. Hübner, Verz. Sehmett., p. 50 (1818).

Perl a ta (part.), Latreille, Fam. Nat. Règne An., p. 468 (1825). - A. Berthold, Latreille, Nat. Fam., p. 473 $(1827)$.

"Morphites" (part.), E. Blanchard, ut supra, p. 454 (1840). - Id., Hist. Ins., v. 2, p. 334, 340 (1845). Morphidae (part.), Kollar, Denkschr. Akad. Wiss. Wien, v. 1, p. 354 (1849). - Morphidae (part.), E. Doubleday (\& J. O. Westwood), Gen. Diurn. Lep., t. 56-59 (1849). Westwood, 1. c., v. 2, p. 332 (1851). - Morphitae (Divis.) (part.), Chenu \& H. Lucas, ut antea (1851-53). - Morphidae (part.), Ménétriés, ut antea (1855). - Morphina (part.), Herrich-Schäiffer, Samml. außereur. Schmett., p. 55 (1858). - Morphidae (part.), Hewitson, Exot. Butterfl., v. 4 (3)-5, t. Dasyophthalma, Paronia etc. (1862-73).

- Herrich-Schäffer, Corr.-BI. Zool.-Mineral. Ver. Regensb.,

v. 16, p. 178 (1862). - Carus-Gerstäcker, Handb. Zool., v. 2, p. 231 (1863). - Hewitson, Equat. Lep., p. 32 (1869). - Morphonides (part.), Burmeister, Rev. Mag. Zool., ser. 3, v. 1, p. 17 (1873). - Morphinae (part.), W. F. Kirby, Entomologist, v. 10, p. 290. - Morphoides (Sous-Fam.), Burmeister, Desscr. Pliys Rép. Argent., จ. 5, pars 1, p. 187, 199 (1878). - Morphida (Subfam.) (part.), Sehmarda, Zool., ed. 2, p. 158 (1878). - Morphidae (part.), W. F. Kirby, Cat. Coll. Diurn. Lep. Hewitson, p. 106 (1879). C. Crüger (sec. Hewitson), Berlin. Ent. Zeit., v. 25, p. 111 (1881). - E. M. Sharpe, Proc. Zool. Soc. London, p. 566 (1890). - Morphinae (part.), Tristan, Ins. Costa Rica, p. 17 (1897). - Morphinae (part.), A. G. Butler, Entomolog., v. 33, p. 90, 189 (1900). - Morphidae, L. D. Cleare, Trans. Ent. Soc. London, v. 74, p. 361 (1926). 
Satyridae (part.), C. \& R. Felder, Wien. Ent. Monatschr., จ. 6, p. 122 (1862). - Iid., Reise Novara, v. 2. II, p. 453 (1866). - Satyrinae (part.), A. G. Butler \& Herb. Druce, Proc. Zool. Soc. London, p. 335 (1874). - W. L. Distant, Proc. Ent. Soc. London, p. XI (1876). - Satyridae (part.), N. D. Riley, Zool. Record, v. 58, pars 11, p. 172 (1921). - Imper. Bureau Entom., 1. c., v. 59 et sequ., p. 301 etc. $(1922-30)$.

Satyromorpha (part.), E. Haase, Deutsch. Ent. Zeit. Lep. (Tris), v. 4, p. 33 (1891). - "Satyromorphen", Satyromorphi, A. Seitz (sec. E. Haase), Ent. Rundschau, v. 45 , p. 3 (1928).

"Pavonides", Boisduval, Considér. Lép. Guatemala, p. 53 (1870). - P., (part.), Capronnier, ut antea (1874\& 81). - Bar, ut antea (1878). - Bönninghausen, ut antea (1896).

Caligoninae, H. Fruhstorfer, ut antea (1912). - Caligonidae, Rothschild, ut antea (1916).

\section{Tribus Brassolidi}

E. Reuter, Acta Soc. Sci. Fenn., v. 22, p. 553 (part.) (1896). H. Stichel, Neue Beitr. syst. Inselktenk., v. 3, p. 59 (1925). Brassolininae (part.), Fruhstorfer, ut antea (1912). - Brassolidae + Caligonidae (part.), Rothschild, ut antea (1916).

\section{Genus Brassolis J. C. Fabr.}

Brassolis (part.), J. O. Fabricius (MS): Illiger, Mag. Insektenk., v. 6, p. 282 (1807). - Latreille, (sec. Fabr.), Gen. Crust. Ins., v. 4, p. 195 (1806-09). - Id., Consid. Gén,, p. 440 (1810). - Id.: Cuvier, Règne An., v. 3, p. 545 (1817), - Jac. Hübner, Verz. Schmett., p. 50 (1818). - Latreille (\& J. B. Godart) Enc. Méth., v. 9, p. 11 (1819). - J. B. Godart, l. c.', p. 456 (1824). - "Brassolide", Latreille, Fam. Règne An., p. 468; p. 469 (sec. Godart) (1825). - Brassolis, Berthold: Latreille Nat. Fam., p. 473, 474 (1827). - II. Burmeister, Ins. Syst. Nat., p. 27 (1829). J. G. Children (sec. Fabr.), Philos. Mag., v. 7, p. 120 (1830).

Griffith-Cuvier, An. Kingdom, v. 15, p. 590 (1832). - "Brassolide", Brassolis, H. Lucas, Hist. Nat. Lép. Exot., p. 142 (1835), - Brassolis, Boisduval (sec. Fabr.), Spec. Gén. Lép., v. 1, p. 82, 97, 118, 153, (1836). - Boisduval-Cuvier, Règne Anim., ed. 3, v. 3, p. 252 (1836). - Verloren, Cat. Ins. Lep. Crimer, v. 2, p. 202 (1837) F. S. Voigt-Cuvier, Règne Anim., v. 5, p. 536 (1839). - E. Blanchard, Hist. An. Artic., v. 3, p. 453 (1840). - D [oyère]: Orbigny, Dict. Hist. Nat., v. 2, p. 728 (1842). - „Brassolide" , Brassolis, Guérin-Méneville, Icon. Règne Anim. Cuvier, Ins., v. 2, p. 487 (1844). - Brassolis, E. Doubleday, List Lep. Brit. Mus., v. 1, p. 109 (1844). - E. Blanchard, Hist. Ins., v. 2, p. 334 (1845). E. Blanchand (\& Doyère): Cuvier, Règne Anim., ed. 4 (Masson, Crochard), จ. 2, Ins., p. 247 (1819). - Hoeven, Handb. Zool., ed. 2, v. 1, p. 398 (1850). - (E. Doubleday \&) J. O. Westwood, Gen. Diurn. Lep., v. 2, p. 350 (part.) (1851). - Chenu \& H. Lucas, Enc. Hist. Nat. Papill., p. 172 (part.) (1851-53). - Wallace, Tr. Ent. Soc. London, n. ser., v. 2, p. 261 (not. biol.) (1854) Ménétriés, Enum. Corp. Anim. Ac. Petropol., Lep., pars 1, p. 39 (1855). - A. Fauvel, Bull. Soc. Linn. Normand., v. 6, (1860-61), p. 140 (1862). - Carus-Gerstäcker, Handb. Zool., p. 232 (1863). 
- H. WV. Bates, Journ. Entom., v. 2, p. 176 (1864). - HerrichSchäffer, Corr.-Bl. Zool.-Min. Ver. Regensb., v. 18, p. 99; vi 19, p. 64 (part.) (1864 \& 65). - Prittwitz, Stett. Ent. Zeit., v. 26, p. 310 (1865). - A. G. Butler, Cat. Diurn. Lep. Fabr., p. 41 (1869). - W. F. Kirby, Cat. Diurn. Lep., p. 125 (1871). - Burmoister, Rev. Mag. Zool., ser. 3, v. 1, p 19, 45 (1873). - Capronnier, Ann. Soc. Ent. Belg., v. 17, p. 28 (1874). - S. H.' Scudder, Histor. Sketch (Proc. Amer. Ac. Sc., v. 10), p. 129 (1875). Wallaco, Geogr. Distrib. Anim., v. 2, p. 472 (1876). - Möschler, Verh. Zool.-Botan. Ges. Wion, v. 26, Abh., p. 321 (1877). - A. G. Butlor, Trans. Ent. Soc. London, p. 114 (1877). - Burmeister, Descr. Phys. Rep. Argent., v. 5, pars 1, p. 194 (1878). - W. F. Kirby, Entomologist, v. 11, p. 26 (part.) (1878). - W. F. Kirby, Cat. Coll. Diurn. Lep. Hewitson, p. 106 (1879). - F. D. Godman \& O. Salvin, Biol. Centr.-Amer., Lep.-Ihhop., v. 1, p, 124 (1881). - C. Crüger (soc. Hewitson), Berlin. Ent. Zeit., v. 25, p. 111 (1881). - Capronnior, Ann. Soc. Ent. Belg., v. 25, p. 101 (1881). - G. C. Champion, Ent. Monthly Mag., v. 19, p. 227 (1883). E. Gerhard, Berl. Ent. Zeit., v. 27, p. 179 (distrib.) (1883). - G. W. Müller, Zool. Jahrb. Syst., v. 1, p. 605 (1886). - L. Glaser, Cat. Etymol. Col. Irop., p. 278 (1887). - O. Staudinger (\& Schatz), Exot. Schmett., v. 1, p. 211 (1887). - Röber, 1. c., v. 2, p. 191, t. 32 (Morphol.) (1859 \& 88). - Richelnann, Tagebl. Deutsch. Naturf. Versamml., 61, p. 68 (1889). - A. Seitz, Stettin. Ent. Zeit., จ. 51, p. 30 (1890). - Hahnel, Deutsch. Ent. Zeit. Lep. (Tris), v. 3, p. 249, 258 (1890). - W. F. Kirby, Handb. Lep., v. 1, p. 199 (1894). - E. Reuter, Acta Soc. Sci. Fenn., v. 22, p. 111, 553 (1896). - H. Stichel, Berlin. Ent. Zeit., v. 46, p. 619 (1901). - Id., Gen. Ins., Fasc. 20, p. 6, t. 1, f. 1 (Morphol.) (1904). Silva, Rolat. Contrib. Hist. Nat. Lep. Brasil (Congr. Scient. Lat.Amer., 1905, v. 3 B) p. 88 (1907). - A. Pagenstecher, Geogr. Verbr. Schmett., p. 413 (1909). - H. Stichel, Tierreich, Lief. 25, p. 6, f. 2 (Morphol.) (1909). - Fruhstorfer: A. Seitz, Großschmett. Erde, v. 5, p. 286 (1912). - Rotlischild, Nov. Zool., v. 23, p. 309 (1916). - Hampson, Novit. Zool., v. 25, p. 385 (1918). Handlirsch: Chr. Schrödor, Handb. Entom., v. 3, p. 9 (1925). Holdhaus, 1. c., v. 2, p. 731 (distrib. geogr.) (1927). - M. Hering, Biol. Schmett., p. 425 (1926). - A. Seitz, Entom. Rundschau, v. 45, p. 4 (1928).

$D$ an a us Subgen. (part.), Linné, Syst. Nat., ed. 10, p. 468, 471 (1758). - Linné-Houttyn, Natuurl. Hist., v. 1. XI, p. 270 (1766-69). - Linné, Syst. Nat., ed. 12, p. 758, 767 (1767). - Danaus (Festivrs) (part.), D. H. Schneider, Nomencl. Entom., p. 35 (1785). - D. (part.), Gmelin-Linné, Syst. Nat., ed. 13, v. 1. V, p. 2282 (1790).

Nymphalis (part.), Latreille, Gen. Crust. Ins., v. 4, p. 194 (1806-09).

"S a t y re" (part.), Latreille, Consid. Gén., p. 440 (1810).

Castnia, Ménétriés, Nouv. Mém. Soc. Nat. Moscou, v. 1, p. $192(1829)$.

Megastes (part.) Boisduval, Consid. Lép. Guatemala, p. $53(1870)$.

sophorae (L.). Papilio (Danaus) s., Linné, Syst. GuayanaNat., ed. 10, p. 471 (1758). - Brassolis s., Colombia, H. Stichel, Gen. Ins., Fasc. 20, p. 7 (1904). Trinidad - Id., Tierreich, Lief. 25, p. 8 (1909). - bis Fruhstorfer, A. Seitz, Großschmett. Erde, Bolivia, จ. 5, p. 287 (1912).

Argentinien 
subsp. sophorae (L.) - Merian, Ins. Su- Guayana, rinam, t. 35, f. 2 (1705) (conf. Burmeister, Venezuela, Abh. Naturf. Gesellsch. Halle, v. 2, 1854, Trinidad, Sitzgsber., p. 63: Brassolis 8., larvae fig. Brasilien dubia). - Kleemann, Beytr. Allgem. Nat.- (part.), Insgesch., v. 1, p. 32, t. 4, f. 1 (1761). ? Argen- Seba, Thesaurus, v. 4, t. 1, 2 (q), t. 15, tinien f. $13,14(1765)$. - Merian-Buchoz, Hist. Ins. Surinam, ed. 3, v. 1, t. 35; v. 2, p. 49 "Sophorae" (1771). - Papilio (Danaus) s., 'inné, ut supra (1758). - P. s., id., (Rydbeck), Amoen. Acad., v. 5, p. 244 (1758). Linné, Mus. Ludov. Ulr., p. 266 (1764). Clerck, Icon. Ins., v. 2, t. 35 , f. 3 (1764). - P. (Danaus) s., Linné-Houttyn, Natuurl. Hist., v. 1. XI, p. 270 (1766-69). - Linné, Syst. Nat., ed. 12, v. 1. II, p. 767 (1767). $P$. s., P. L. S. Mülller, Naturs. Linné, v. 5. I, p. 597 (1774). - J. 'C. Fabricius, Syst. Ent., p. 483 (cit. part.) (1775). - (P. F. Gmelin \&) Christman, Onomatol. Med., v. 6, p. 151 (1775). - P. (Danaus) s., Goeze, Ent. Beytr., v. 3. I, p. 193 (cit. part.) (1779). - "Sophorae", Pap. s., P. Cramer, Pap. Exot., v. 3, p. 105, t. 253, f. A-C; p. 176 (1779 \& 80). - P. s., J. O. Fabricius, Spec. Ins., v. 2, p. 58 (cit. part.) (1781). C. Stoll, Essai Syst. Iep. (P. Cramer, Pap. Exot., v. 4, App.) p. 8, Nr. 25 (1782). $P$. (Danaus Festivus) s., D. H. Schneider, Nomencl. Entom., p. 35 (1785). - P. s., J. C. Fabricius, Mant. Ins., v. 2, p. 28 (cit. part.) (1787). - P. (Danaus) 8., GmelinLinné, Syst. Nat., ed. 13, v. 1. V, p. 2282 (cit. part.) (1790). - P. s., O. Stoll, Suppl. Cramer, Pap. Exot., t. 3, f. 2A, B (larva, chrysal.) (1791). - Jung, Alphab. Verz. Schmett., จ. 2, p. 205 (1792). - J. O. Fabricius, Int. Syst., v. 3. I, p. 150; Index alphab., p. 128 (1793 \& 96). - (Jablonsky \&) Herbst, Naturs. Ins., Schmett., v. 6, p. 57 (cit. part.), t. 129, f. 4,5 (o); t. 130 , f. $1\left(\sigma^{7}\right)(1793)$. - Fr. Weber, Nomenal. Entom., p. 101 (1795). - "P. du sophord" (!), Tubleau Encycl. Méth., v. 18, t. 26, f. 2, 2bis; f. 1A, B (larra, chrysal.) (1797). - Pap. sophorae, Latreille, Iist. Crust. Ins., v. 3, p. 392 (1802). - Thunberg, Mus. Nat. Ac. Upsal., pars 23, p. $9(1804)$ - G. Shaw, Gen. Zool., v. 6. I, p. 212, t. 69, f. 1 (1806). - Turton, Gen. Syst. Nat. Linné, v. 3. II, p. 117 (1806). - P. (Brassolis) s., Latreille, Gen. Crust. Ins., v. 4, p. $195(1806-09)$ - - B. (P.) s., J. C. Fabricius (MS): Tlliger, Mag. Insektenk., v. 6, p. 282 (1807). - P. s., Latreille, Consid. Gén., p. 440 (1810). - Brassolis s., Oken, Lehrb. Naturg., v. 1, p. 746 (1815). - Jac. Hübner, Verz. Schmett., p. 50 (1816). 
- Pap. 8., Latreille: Cuvier, Règne Anim., v. 3, p. 545 (1817). - Brassolis 8., (Latreille \&) J. B. Godart, Enc. Méth., v. 9, p. 457 (1824). - J. G. Children (sec. Fabr.), Philos. Mag., v. 7, p. 120 (1830). - Lacordaire, Ann. Soc. Ent. France, v. 2, p. 396 (1833). - H. Lucas, Hist. Nat. Lép. Exot., p. 142, t. 78, f. 2 (1835). - Boisduval: Cuvier, Règne, Anim., ed. 3, v. 3, p. 252 (1836). - Verloren, Cat. Ins. Cramer, v. 1, p. 97,152 ; v. 2, p. 202 (1837). - E. Blanchard, Hist. An. Artic., v. 3, p. 453, t. 15 , f. 3 (1840). - D [oyère]: Orbigny, Dict. Hist. Nat., v. 2, p. 728 (1842). - E. Doubleday, List Lep. Brit. Mus., v. 1, p. 119 (1844). - Erichson: Schomburgk, Guiana, v. 3, p. 599 (1848), - B. sophona, Blanchard (\& Doyère); Cuvicr, Règne Auim., ed. 4 (Masson, Crochard), v. 2, Ins., p. 247 , t. 141 , f. 2 (Q), f. 2 a, b (Caput, antenn.), f. 2c, d (larva, chrysal.) (1849).B. sophorae, I. Doubleday (\& Westwood), Gen. Diurn. Lep., t. 59, f. 2 (ㅇ) (1849); Westwood, 1. c., v. 2, p. 351 (1851). Hoeven, Handb. Zool, ed, 2, v. 1, p. 389 (1850). - Chenu \& H. Lucas, Enc. Hist. Nat., Pap. Diurn., p. 173, f. 284 (1851-53). - Pap. 8., „Saphorae (!), Sepp. Surinam. Vlinders, p. 309, t. 143 (1852). - Wallace, Tr. Int. Soc. London, n. ser., v. 2, p. 261 (1854). - Brassolis 8., Ménétriés, Lnum. Corp. Anim. Ac. Petropol., Lep., pars 1, p. 39 (1855). - A. Fauvel, Bull. Soc. Linn. Normand, v. 6 (1860-61), p. 140 (1862). - Herrich-Schäffer, Corr.-B1. Zool.-Mineral. Ver. Regensb., v. 19, p. 61 (1865). - Prittwitz, Stett. Ent. Zeit., v. 26, p. 310 (not. biol.) (1865). - A. G. Butler, Cat. Diurn. Lep. Fabr., p. 41 (1869). - W. F. Kirby, Pap. s. (Brassolis) Tr. Ent. Soc. London, p. 143 (determ. typ. Linnaei) (1870). B. 8., id., Cat. Diurn. Lep., p. 125; Suppl., p. 716 (1871 \& 77). - Orotch (sec. Fabr.), Cist. Ent., v. 1, p. 66 (1872). - Burmeister, Rev. Mag. Zool., ser. 3, v. 1, p. 46, t. 1, f. 12, 14 (Metamorph.) (1873). - A. G. Butler, Trans. Ent. Soc. London, p. 114 (1877). - Möschler, Verh. Zool.-Bot. Ges. Wion, v. 26 (1876), p. 321 (1877). - ? Burmeister, Descr. Phys. Rep. Argent., v. 5, pars 2, p. 194 (1878). - W. F. Kirby, Entomologist, v. 11, p. 26 (1878). - Id., Cat. Coll. Diurn. Lep. Hewitsan, p. 106 (1879). Chr. Aurivillius, Svenska Ak. Handl., n. ser., v. 19, Nr. 5, p. 74 (1882). - O. Staudinger (\& Schatz), Exot. Schmett., v. 1, t. 71, p. 211 (1886 \& 87). - I. Glaser, Cat. Etymol. Col. Lep., p. 278 (1887). Hahnel, Deutsch. Ent. Zeit. Lep. (Iris), 
v. 3, p. 249 (1890). - T. A. Ohapman, Ent. Rec. Journ. Var., v. 6, p. 131, t. 2, f. 12,20 (Metam.) (1895). - E. Reuter, Acta Sac. Sei. Fenn., v. 22, p. 111 (1896). - Bönninghausen, Verh. Ver. Naturw. Unterhalt. Hamburg, v. 9, p. 40 (1896). B. s. s., H. Stichel, Gen. Ins., Fasc. 20, p. 7 (1904). - Id., Tierreich, Lief., 25, p. 10 (1909). - B. 8., A. G. Weeks, Tll. Diurn. Lep., v. 2, p. XI (1911). - F. W. Urich \& Guppy, Board Agric. Trinidad, Circ. Nr. 5, p. 19, t. 1 (Biol.) (1911). - Eecke, Not. Leiden Mus., v. 34, p. 257, f. 2 (Q) (1912). - B. s. s., Fruhstorfer: $\Lambda$. Seitz, Großschmett. Erde, v. 5, t. 62 a; p. 287 (1911 \& 12). - B. 8., W. J. Kaye, Trans. Ent. Soc. Lond., p. 547 (1914). - Cleare, Bull. Entom. Res. London, v. 6, p. 273 , f. 1 (larva, chrysal.), t. 8-10 (Biol.), t. 10, f. 1 (P), f. 2 (ova) (1915). - B. 8. s., Rothschild, Nov. Zool., v. 23, p. 314, t. 6, f. 7, 9 (larva, chrysal.) (1916). - Hampson, 1. c., v. 25, p. 385 (1918). - ? B. s., P. Köhler, Fauna Argent., par's 1 (Zeit. wiss. Ins.-Biol., v. 18, Bciheft) p. 21 (1923).

Papilio xanthus (non Linné), (Jablonsky \&) Herbst, Naturs. Ins., Schmett., v. 6, p. 48 (part. in cit.) (1793).

rufescente-fuscus (Goczc). Papilio (Danaus) $r-t$. , Goeze, Entom. Bcytr., v. 3. I, p. 223 (1779).

subsp. lurida Stich. B. s. luridus, H. Stichel, Berlin. Ent. Zeit., v. 46, p. 520 (1902). - B. s. luvida, id., Gen. Ins., Fasc. 20, p. 7 (1904). - Id., Tierreich, Lief. 25, p. 11 (1909). - Fruhstorfer: A. Seitz, Großschmett. Erde, v. 5, p. 287 (1912). - Eecke; Not. Leiden Mus., จ. 34, p. 257, f. 1 (ㅇ, forma indiv.) (1912).

subsp. ardens Stich. B. s. a., H. Stichel, Ins. Börse, v. 20, p. 389 (1903). - Id., Gen. Ins., Fasc. 20, p. 7, t. 3, f. 1 (1904). - Id., Tierreich, Lief. 25, p. 12 (distrib. part.) (1909). - Fruhstorfer: A. Seitz, Großschmett. Erde, v. 5, p. 287 (dist. part.) (1912). - H. Stichel, Neue Beitr. syst. Ins.kunde, v. 3, p. 60 (1925).

subsp. philom ela Stich. B. 8. p., H. Sti- Ecuador chel, Neue Beitr. syst. Ins.kunde, v. 3, p. 60 (Macas) (1925).

? B. sophorae (non Pap. s. L.), Dognin, Lép. Loja, p. 72 (1894).

subsp. $L$ a u $\operatorname{renti}$ i Stich. B. s. l., H. Stichel, Minas Geraes Neue Beitr. syst. Ins.kunde, v. 3, p. 61 (Juiz de (1925).

Fora)

subsp. vulpeculus Stich. B. s. v., H. Sti- Paraguay, ehel, Berlin. Ent. Zeit., v. 46, p. 520 (1901). Argentinien - Id., Gen. Ins., Fasc. 20, p. 7 (1904). - (Misiones) 
Id., Tierreich, Lief. 25, p. 11 (1909). - H. Fruhstorfer: A. Seitz, Großschmett. Erde, v. 5, p. 287 (1912). - W. Rothschild, Nov. Zool., v. 23, p. 314 (forma communis desertorum) (1916). - B. v., P. Köhler, Fauna Argent., pars 1 (Zeit. wiss. Ins.-Biol., v. 18, Beiheft), p. 21 (1923). - B. sophorae v., H. Stichel, Neue Beitr. syst. Ins.kunde, $v$. 3, p. 59 (1925). - Talbot, Bull. Hill Mus., v. 2, p. 199 (1928).

? B. sophorae (non Pap. s. L.), P. H. Gosse, Entomologist, v. 13, p. 201 (1880).

Haenschi Stich. B. h., H. Stichel, Berlin. Int.Zeit., v. 46, p. 520 (1902). - Id., Gen. Ins., Fasc. 20 , p. 8 (1904). - Id., Tierreich, Lief. 25, p. 15 (1909).

subsp. $H$ a e $n$ s $c h i$ Stich. $B . h$. $h$., H. Stichel, ut antea, t. 8, f. 2 (1902). - Id., ut antea (1904 \& 1909). - B. astyra h., Fruhstorfer: A. Seitz, Großschmett. Irde, v. 5, p. 287 (1912). - B. h. h., H. Stichel, Neue Beitr. syst. Ins.kunde, v. 3, p. 62 (1925).

subsp. $r$ ufescen \& Rothsch. B. astyra r., W. Nord-Peru Rothschild, Nov. Zool., v. 23, p. 315 (1916). - B. haenschi r., H. Stichel, Neue Beitr. syst. Ins.kunde, v. 3, p. 62 (1925).

subsp. maritima Stich. B. h. m., H. Stichel, Venezuela, Ins. Börsc, v. 30, p. 389 (1903). - Id., P NordGen. Ins., Fasc. 20, p. 8 (1904). - Id., Brasilien

Tierreich, Lief. 25, p. 16 (1909). - B. (sec. Rosa) astyra m., Fruhstorfer: A. Seitz, Großschmett. Erde, v. 5, p. 287 (1912). - B. haenschi m., H. Stichel, Neue Beitr. syst. Ins.kunde, v. 3 , p. 62 (1925). - ? B. astyra m., Rosa, Bol. Mus. Nacion. Rio Janeiro, v. 2, Nr. 4, p. 24 (1926).

Ecuador,

Nord-Peru,

Venezuela

Ost-

Ecuador

- astyra God. B. a., (Latreille \&) J. B. Godart, Enc. Méth., v. 9, p. 457 (1824). - H. Stichel, Gen. Ins., Fasc. 20, p. 7 (1904). - Id., Tierreich, Lief. 25, p. 13 (1909). - Fruhstorfer: A. Seitz, Großschmett. Erde, v. 5, p. 287 (part.) (1912).

subsp. a s ty ra God. ? - Seba, Thesaurus, v. 4, Espirito t. 20 , f. $15,16(1765)$ (sec. Aurivillius, $1882,=B$. sophorae, sed errans puto). B. a., (Latreille \&) J. B. Godart, Enc. Méth., v. 9, p. 457 (1824). - GriffithCuvier, Anim. Kingdom, v. 15, t. 47, f. 4, f. 3 (larva) (1832). - Boisduval, Speo. Gén. Lép., v. 1, Expl. pl., p. 4, t. 13, f. 2 (1836). - Guérin-Ḿ́neville, Iconogr. Règne Anim., v. 2, Ins., p. 487, t. 79, f. 4 (182944). - D [oyère]: Orbigny, Dict. Hist. Nat. v. 2, p. 728 (1842). - E. Doubleday, List Lep. Brit. Mus., v. 1, p. 119 (1844). E. Doubleday (\& J. O. Westwood), Gen. Diurn. Lep., t. 59, f. 1 ( $\left(^{\top}\right)(1849)$; Westwood, 1. c., . 2 , p. 351 (1851). - Chenu \& H. Lucas, Enc. Hist. Nat. Papill., p.

Santo-

Santa

Catharina.

Nord-

Argentinien 
173 (1851-53). - Ménétriés, Enum. Corp. Anim. Ac. Petrop., Lep., pars 1, p. 39 (1855). - Herrich-Schäffer, Corr.-Bl. Zool.Min. Ver. Regensb., v. 19, p. 64 (1865). - W. F. Kirby, Cat. Diurn. Iep., p. 125 (1871). - Capronnier, Ann. Soc. Ent. Belg. v. 17, p. 28 (Phaenol.) (1874). - W. F. Kirby, Entomologist, v. 11, p. 26 (1878). Id., Cat. Coll. Diurn. Lep. Hewitson, p. 106 (1879). - Capronnier, Ann. Soc. Ent. Belg., v. 25, p. 101 (Phaenol. (1881). Dukenfield-Jones (\& F. Moone), Proc. Liverp. Lit. Soc., Nr. 36, p. 331, 337, t. 3, f. 2 (chrysal.) (1882). - W. Müller, Zool. Jahrb. Syst., v. 1, p. 602 (Biol.) (1886). - L. Glaser, Cat. Etymol. Col. Lep., p. 278 (1887). - O. Staudinger (\& Schatz), Exot. Schmett., v. 1 , t. 71 ; p. 211 (1886 \& 87). - Sommerfeld, Ins.-Börse, v. 13, p. 69 (1896). Bönninghausen, Verlı. Ver. Naturw. Unterh. Hamburg, v. 9, p. 40 (1896). - B. a. a., H. Stichel, ut antea (1904 \& 09). - B. a., Silva, Relat. Contrib. Hist. Nat. Lep. Brasil (Congr. Scient. Lat.-Amer., 1905, v. 3B), p. $88(1907)$. - B. a. a., Fruhstorfer: A. Seitz, Großschmett. Erde, v. 5, p. 287 (non t. $62 a:=$ B. a. philocala) (1912). - W. Rothschild, Nov. Zool., v. 23, p. 315, t. 6, f. 8 (larva) (1916). - B. a., P. Köhler, Fauna Argent., pars 1 (Zeit. wiss. Ins.-Biol., v. 18, Beiheft), p. 21 (1923). - H. Stichel, Neue Beitr. syst. Ins.kunde, v. 3, p. 61, 62 (1925). - B. a., Le Cerf, Enc. Ent., ser. B3, Lep., v. 1, p. 141 (Anatom.) (1926). Zikán, Ent. liundschau, v. 45, p. 10 (1928).

Langsdorfi (Mén.) Castnia l., Ménétriés, Nouv. Mém. Soc. Nat. Moscou, v. 1, p. 192, t. 5 (1829).

a styalus (Burm.) Brassolis a., Burmeister, Abh. Naturf. Ges. Halle, v. 2, SB., p. 58 (1854). - Id. Rev. Mag. Zool., ser. 3, v. 1, p. 46 (Biol.) (1873).

forma $d i m i d i a t a$ Fruhst. B. a. a. forma $d$. , Typ.: Santa Fruhstorfer: A. Seitz, GroBschmett. Erde, v. Catharina 5, p. 287 (1912). - B. a. forma d., P. Köhler, Fauna Argent., pars 1 (Zeit. wiss. Ins.Biol., v. 18, Beiheft), p. 21 (1923). - H. Stichel, Neue Beitr. syst. Ins.kunde, v. 3, p. 50 (1925).

subsp. $p$ hil o c a l a Stich. B. a. p., H. Stichel, Ins.-Börse, v. 21, p. 21 (1901). - Id., Gen. Ins., Fasc. 20, p. 7 (1904). - Id., Tierreich, Lief. 25 , p. 15, f. 3 (1909). - Fruhstorfer: A. Seitz, Großschmett. Erde, v. 5, p. 287, t. $62 \mathrm{a}$ (astyra O') (1912).

isthmia Bat. B. i., H. W. Bates, Ent. Monthly Mag., Mittelv. 1, p. 164 (1864).

Amerika, Colombia, Bolivia 
subsp. isthmia Bat. B. i., H. W. Bates, ut antea (1864). - Herrich-Schäffer, Corr.Bl. Zool.-Min. Ver. Rogensb., v. 19, p. 64 (1865). - W. F. Kirby, Cat. Diurn. Lep., p. 125 (1871). - F. D. Godman \& O. Salvin, Biol. Centr.-Amer., Lep.-Thop. v. 1 , p. 125 , t. 12 , f. $5-8$ (1881). - O Staudinger (\& Schatz), Exot. Schmett., $\nabla$. 1, p. 211 (1887). - Röber, l. c., v. 2, t. 32 (Morphol.); p. 191 (1888 \& 89). - H. Stichel, Gen. Ins., Fasc. 20, p. 8 (1904). Id., Tierreich, Lief. 25, p. 17 (1909). H. F. Schultz, Proc. Ent. Soc. Washington, v. 10 (1908), p. 164 (Biol.) (1909). - Fruhstaffer: A. Seitz, Großschmett. Erde, v. 5, p. 287 (1912). — G. H. Dyar, Proc. U. S. Nat. Mus., จ. 47, p. 144 (1914). - B. i. i., W. Rothschild, Nov. Zool., v. 23, p. 315 (1916). - B. i., Dunn, Tourn. Econ. Ent., v. 10, p. 473, t. 22, 23 (1917). - B. i. i., H. Stichel, Neue Beitr. syst. Ins.kunde, v. 3, p. 62 (1925).

subsp. boliviana Rothsch. $B$. i. b., W. Rothschild, Nov. Zool., v. 23, p. 315 (1916). granadensis Stich. B. g., H. Stichel, Berlin. Ent. Zeit., v. 46 , p. 521, f. 4 (1902). - Id., Gen. Ins., Fasc. 20, p. 8 (1904). - Id., Tierroich, Lief. 25 , p. 18 , f. 4 (1909). Fruhstorfer: A. Seitz, Großschmett. Erde, v. 5 , t. $62 \mathrm{a}$, p. 287 (1911 \& 12). - B. isthmia g., W. Rothschild, Nov. Zool., v. 23, p. 315 (1916). - B. g., Fassl, Ent. Rundschau, v. 35 , p. 31 (1918), - H. Stichel, Neue Beitr, v. 3, p. 62 (1925).

$B$. astyrahaenschi (non B. h. Stich.), Talbot, Bull. Hill Mus., v. 3, p. 143, t. 3, f. 2 (O) (1929).

ornamentalis Stich. $B$. o., H. Stichel, Ent. Zeit. Guben, v. 20, p. 209, fig. (1906). - Id., Tierreich, Lief, 25, p. 19, f. 5 (1909). - Fruhstorfer: A. Seitz, Großschmett. Erde, v. 5, p.

\section{Ost-Bolivia}

Colombia,

Ecuador

Panama

Taboga Insel, Nicaragua,

Colombia

(Cauca) 288 (1912).

\section{Genus Penetes E. Doubl.}

Penetes (Boisduval MS), E. Doubleday (\& Westwood), Gen. Diurn. Lep., t. 58 (1849); J. O. Westwood, 1. c., v. 2, p. 347 (1851). - Chenu \& H. Lucas, Enc. Hist. Nat., Pap. Diurn., p. 171 (1851-53). - H. W. Bates, Journ. Enlom., v. 2, p. 164 (1854). - Herrich-Schäffer, Corr.-Bl. Zool.-Min, Vor. Regensb., จ. 18, p. 99 ; v. 19, p. 64 (1864 \& 65). - W. F. Kirby, Cat. Diurn: Lep., p. 127 (1871). - S. H. Scudder, Histor. Sketeh (Proc. Amer. Ac. Sc., v. 10) p. 243 (1875). - Wallace, Geogr. Distrib. Anim.; v. 2, p. 472 (1876). - W. F. Kirby, Entomologist, v. 11, p. 27 (1878). - Id., Cat. Coll. Diurn. Lep. Hewitson, p. 106 (1879). - C. Crüger (sec. Hewitson), Berlin. Ent. Zeit., v. 25, p. 111 (1881). - E, Gerhard, 1. c., v. 27, p. 179 (distrib.) (1883). - L. Glaser, Cat. Etymol. Col. Lrep., p. 306 (1887). - O. Staudinger (\& Schatz), 
Exot. Schmett., v. 1, p. 214 (1887). - Röber, 1. c., v. 2, to 32 (Morphol.); p. 192 (1838 \& 89). $\rightarrow$ H. Stichel, Gen. Ins., Fasc. 20, p. 12, t. 1, f. 4 (Morphol.) (1904). - 1 . Pagenstecher, Geogr. Verbr. Schmetterl., p. 413 (1909). - H. Stichel, Tierreich, Licf. 25, p. 36, f. 9 (Morphol.) (1909). - Fruhstorfer: $\Lambda$. Scitz, Großschmett. Erde, v. 5, p. 283 (1912).

pamphanis Doubl. P. p. (Boisduval MS), E. Doub- Minas Geraes, leday (\& Westwood), Gen. Diurn. Lep., t. Espirito 58 , f. 1 (1849); J. O. Westwood, 1. c., v. 2, Santop. 348 (1851). - Chenu \& H. Lucas, Enc. Rio Grande Hist. Nat. Pap. Diurn., p. 171, f. 282 do Sul (1851-53). - Herrich-Schäffer, Corr.-Bl. Zool.-Min. Ver. Regensburg, v. 19, p. 64 (1865). - W. F. Kirby, Cat. Diurn. Lep., p. 127 (1871). - Id., Entomologist, v. 11, p. 27 (1878). - Id., Cat. Coll. Diurn. Lep. Hewitson, p. 106 (1879). - O. Staudinger (\& Schatz), Exot. Schmett., v. 1, t. 72; p. 214 (1886 \& 87). - L. Glaser, Cat. Etymol. Col. Lep., p. 306 (1887). - Röber: O. Staudinger \& Schatz, Exot. Schmett., v. 2, t. 32 (Morphol.); p. 192 (1888 \& 89). - Bönninghausen, Verh. Ver. Naturw. Unterhalt. Hamburg, v. 9, p. 40 (1896). - H. Stichel, Gen. Ins., Fasc. 20, p. 12 (1904). Id., Tierreich, Lief. 25, p. 38 (1909). Fruhstorfer: A. Seitz, Großschmett. Erde, v. 5 , t. 67 b, p. 298 (1911 \& 12). - Schwanwitsch, Proc. Zool. Soc. London, p. 522, t. 1 3, f. 20 (Physiol.) (1924).

\section{Tribus Naropidi}

H. Stichel, Neue Beitr. syst. Ins.kunde, v. 3, p. 59 (1925). $\neg$ Caligonlinae (Subfam.) (part.), Fruhstorfer: A. Seitz, Großschmett. Erde, v. 5, p. 290 (1912). - Caligonidae (Fam.) (part.), W. Rothschild, Nov. Zool., v. 23, p. 309 (1916).

\section{Genus Narope E. Doubl.}

E. Doubleday (\& Westwood), Gen. Diurn. Lop., t. 50 (1849); J. .O Westwood (Boisduval IIS), 1. c., v. 2, p. 348 (1851). Chenu \& H. Lucas, Enc. Hist. Nat., Pap. Diurn., p. 172 (1851-53). - Ménétriés, Enum. Corp. Anim. Ac. Petrop., Lep., pars 1, p. 39 (1855). - Herrich-Schäffer, Corr,-Bl. Zool.-Min. Ver. Regensb., v. 19, p. 99 ; v. 19 , p. 65 (1861 \& 65). - H. W. Bates, Journ. Entom., v. 2, p. 176 (1864). - W. F. Kirby, Cat. Diurn.

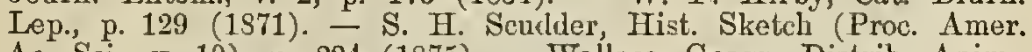
Ac. Sci., v. 10), p. 224 (1975). - Wallace, Geogr. Distrib. Anim. v. 2, p. 472 (1876). - Möschler, Verh. Zool.-Botan. Ges. Wien, Abh., p. 322 (1877). - F. Müller, Jena. Zeit. Naturw., v. 11, p. 102 (Physiol.) (1877). - W. F. Kirby, Entomologist, v. 11, p. 27 (1878). - C. Crüger (sec. Hewitson), Berlin. Ent. Zeit., v. 25, p. 110 (1881). - F. D. Godman \& O. Salvin, Biol. Centr.-Amer., Lep.Rhop., v. 1, p. 139 (1881). - E. Gerhard, Berlin. Ent. Zeit. v. 27, p. 179 (distrib.) (1883). - L. Glaser, Cat. Etymol. Lep. Col., p. 302 (1887). - O. Staudinger (\& Schatz), Exot. Schmett., v. 1, p. 
218 (1887). - Röber, l. c., v. 2, t. 32; p. 191 194 (1888 \& 89). Richelmann, Tagebl. Deutsch. Naturf. Versamml., 61, p. 69 (1889). - H. Stichel, Gen. Ins., Fasc., 20, p. 13, t. 1, f. 5 (Morphol.) (1904). - A. Pagenstecher, Geogr. Verbr. Schmett., p. 413 (1909). H. Stichel, Tierreich, Lief. 25, p. 39, f. 10, 11 (Morphol.) (1909). - H. Fruhstorfer: A. Seitz, Großschmett. Erde, v. 5, p. 327 (1912). - H. Stichel, Neuo Beitr. syst. Ins.kunde, v. 1, p. 7 (1916). - Holdhaus: Ohr. Schröder, Handb. Entom., v. 2, p. 743 (distrib. gcogr.) (1927).

Naropina, Röber, Int. Ent. Zeit., v. 23, p. 326 (1929).

\section{Sectio Pilopoecili}

H. Stichel, Gen. Ins., Fasc. 20, p. 14 (1904), - Id., Tierrcich, Lief. 25, p. 42 (1909).

\section{a. Cohors Cyllastroformes}

do - H. Stichel, ut antea (1904 \& 09). Gen. Diurn. Lep., t. 50, f. 4 (1849). - H. Guayana, Stichel, Gen. Ins., Fasc. 20, p. 14 (1904). - Venezuela, Id., Tierreich, Lief. 25, p. 42 (1909). - PeruFrulıstorfer: A. Seitz, Großschmett. Erde, Südv. 5, p. 328 (1912).

Brasilien,

Nord-

Argentinien

subsp. cyllastros Doubl. N. c., E. Double- Guayana, day, ut antea (1849); J. O. Westwood, Venezuela, ibid., v. 2, p. 349 (1851). - Chenu \& H. Brasilien, Lucas, Enc. Hist. Nat., Pap. Diurn., p. 172, Nordf. 283 (1851-53). - Ménétriés, Enum. Corp. Anim. Ac. Petrop., Lep., pars 1, p. Argentinien 39 (1855). - Herrich-Schäffer, Corr.-Bl. Zool.-Min. Ver. Regensb., v. 19, p. 65 (1865). - W. F. Kirby, Cat. Diurn. Lep., p. 129 (1871). - Möschler, Verh. Zool.-Bot. Ges. Wien, v. 26, Abh., p. 322 (1877). - W. Müller, Zool.Jahrb. Syst., v. 1, p. 601 (1886). - L. Glaser, Cat. Etymol. Col. Lep., p. 302 (1887). - O. Staudinger (\& Schatz), Exot. Schmett., v. 1, t. 76; p. 218 (1886 \& 87). Röber, l. c., v. 2, t. 32 (Morphol.); p. 194 (1888 \& 89). - Bönninghausen, Verh. Ver. Naturw. Unterh. Hamburg, v. 9 (1894-95), p. 39 (1896). - N. c. c., II. Stichel, Ins.Börse, v. 21, p. 197 (1904). - Id., Gen. Ins., Fasc. 20, p. 14 (part.) (1904). - Id., Tierreich, Lief. 25, p. 43 (cit. part.) (1909). - Truhstorfer: A. Seitz, Großschmett. Erde, v. 5 , t. 62d; p. 328 (1911 \& 12). - N. c., H. Stichel, Neue Beitr. syst. Ins.kunde, v. 1, p. 7 (1916). - N. cyllastrus, P. Köhler, Fauna Argent., pars 1 (Zeit. wiss. Ins.-Biol., v. 18, Beiheft), p. 21 (1923). - N. cyllastros, Zikán, Ent. Rundschau, v. 45, p. 11 (1928).

cyllene Feld. N. c., C. \& R. Felder, Wien. Ent. Monatschr., v. 3, p. 270 (ㅇ) (1859). - 
Herrich-Schäffer, Corr:-Bl. Zool.-Min. Ver. Regensb., v. 19, p. 65 (1865). - W. F. Kirby, Cat. Diurn. Lep., p. 129 (1871).

forma stygius Stauding. N. 8., O. Staudin- Typ.: ger (\& Schatz), Exot. Schmett., p. 218 (P) Amazonas (1886). - N. cyllastros s., Fruhstorfer: A. (Fontebea) Seitz, Großschmett. Erdo, v. 5, p. 328 (1912). - N. 8., H. Stichel, Neue Beitr. syst. Ins.kunde, v. 1, p. 7 (? cyllastros forma) (1916). N. cyllas tro s c., H. Stichel, ut antea (part. in cit.) (1904 \& 09).

subsp. $t$ e s $t a$ c e a Godm. \& Salv. N.t., F. D. Panama Godman \& O. Salvin, Ann. Mag. Nat. Hist., (Chiriqui) ser. 5, v. 2, p. 259 (1878). - Iid., Biol. Centr.-Amer., Lep.-Rhop., v. 1, p. 139, t. 15, f. 4-6 (1881). - O. Staudinger (\& Schatz), Exot. Schmett., v. 1, p. 218 (1887). $-N$. cyllastros t., H. Stichel, Ins.-Bö̀se, v. 21, p. 197 (1904). - Id., Gen. Ins., Fasc. 20, p. 15 (1904). - Id., Tierreich, Lief., 25, p. 44 (1909). - Fruhstorfer: A. Seitz, Großschmett. Erde, v. 5, p. 328 (1912).

subsp. de n t ic u l a ta Talb. N.cyllastros den- Mato Grosso ticulatus, Talbot, Bull. IIill Mus., v. 2, p. 200, t. 8, f. 13 (1928) (subsp. dubia).

subsp. s y lla $b$ us Stauding. N. s., O. Stau- Peru, dinger (\& Schatz), Exot. Schmett., v. 1, p. Bolivia 218 (1887). $-N$. anartes syllabus, Fruhstorfer: A. Seitz, Großschmett. Ende, v. 5, p. 329 (1912). - H. Stichel, Neue Beitr. syst. Ins.kunde, v. 1, p. 7 (cyllastros subsp.) (1916).

$N$. anartes (non Hewitson), H. Stichel, Gen. Ins., Fasc. 20, p. 15 (part. in cit.) (1904). - Id., Tierreich, Lief. 25, p. 46 (ut antea) (1909).

- cyllarus Westw. N. c., (E. Doubleday \&) J. O. MittelWestwood, Gen. Diurn. Lep., v. 2, p. 349 nota (1851). - Herrich-Schäffer, Corr.-Bl. Zool.-Min. Ver. Regensb, v. 19, p. 65 (1865). W. F. Kirby, Cat. Diurn. Lep., p. 129 (1871). - O. Staudinger (\& Schatz), Exot. Schmett., v. 1, p. 218 (1887). - Bönninghausen, Verh. Ver. Naturw. Unterh. Hamund SüdBrasilien (Espirito Santo-Rio Grande do Sul) burg, v. 9, p. 39 (1896). - H. Stichel, Gen. Ins., Fasc. 20, p. 15, t. 4, f. 1 (1904). - Zikán, Ent. Rundschau, v. 45, p. 11 (1928).

anartes Hew. N. a., Hewitson, Descr. Butterfl. ColombiaBolivia, p. 9 (1874). Peru

subsp. a nartes Hew. $N$. a., Hewitson, ut Bolivia, antea (1874). - W. F. Kirby, Cat. Diurn. Peru Lep., Suppl., p. 717 (1877). - O. Staudinger (\& Schatz), Exot. Schmett., v. 1, p. 218 (1887). - H. Stichel, Gen. Ins., Fasc. 20 , p. 15 (cit. part.) (1904). - Id., Tierreich, Lief. 25, p. 46 (cit. part.) (1909). H. Fruhstorfer: A. Seitz, Großschmett. Erde, 
v. 5, p. 329 (1912). - H. Stichel, Neue

Beitr. syst. Ins.kunde, v. 1, p. 7 (1916).

forma disyllus Fruhst. $N$. sarastro $d$. , Typ.: HuanFruhstorfer: A. Seitz, Großschmett. Erde, v. cabamba 5 , p. $329(1912)$. $-N$. d., H. Stichel, Neue Beitr. syst. Ins.kunde, v. 1, p. 7 (anartes forma) (1916).

forma marmorata Schaus. N. m., Schaus, Typ.:

Proc. U. S. Nat. Mus., v. 24, p. 390 (1902). Bolivia

- H. Stichel, Neue Beitr. syst. Ins.kundo, v. 1 , p. 7 (anartes aut syllabus forma) (1916).

$N$. anartes (non Hew.), H. Stichel, Gen. Ins., Fasc. 20, p. 15 (part. in cit.) (1904). - Id., Tierreich, Lief. 25, p. 46 (ut antea) (1909).

subsp. sarastro Stauding. N. s., O. Stau- Colombia, dinger (\& Schatz), Exot. Schmett., v. 1, t. Ecuador $76 ;$ p. 218 (1886 \& 87). - H. Stichel, Ins.Börse, v. 21, p. 21 (1904). - Id., Gen. Ins., Fasc. 20, p. 15 (1904). - Id., Tierreich, Lief. 25, p. 45 (1909). - Frubstorfer: A. Seitz, Großschmett. Erde, v. 5, p. 329 (1912). - H. Stichel, Neue Beitr. Syst. Ins.kunde, v. 1, p. 7 (anartes subsp.) (1916). - Fassl, Ent. Rundschau, v. 35, p. 31 (1918). - M. Hering \& W. Hopp, Deutsch. Ent. Zcit. Iris, v. 39, p. 193 (1925).

\section{b. Cohors Nesopiformes}

H. Stichel, Gen. Ins., Fasc. 20, p. 15 (1904). - Id., Tierreich, Lief. 25, p. 48 (1909). - Id., Neue Beitr. syst. Ins.kunde, v. 1, p 7 (suppl. diagnos.) (1916).

nesope Hew. N. n., Hewitson, Equat. Lep., p. 32 Ecuador (1869). - W. F. Kirby, Cat. Diurn. Lep., p. 129 (1871) - H. Stichol, Gen. Ins., Fasc. 20, p. 15 (1909). - Id., Tierreich, Lief. 25, p. 48 (1909). - Fruhstorfer: A. Seitz, Großschmett. Erde, v. 5, p. 329 (1912).

cyllabarus Westw. N. c., (E. Doubleday \&) J. O. Guayana, Westwood, Gen. Diurn. Lep.s v. 2, p. 349, Amazonas, nota (1851). - Herrich-Schäffer, Corr.-Bl. Colombia, Zool.-Min. Ver. Regensb., v. 19, p. 65 (1865). Bolivia, - W. F. Kirby, Cat. Diurn. Lep., p. 129 ? Mexiko (1871). - O. Staudinger (\& Schatz), Exot. Schmett., v. 1, p. 218 (1887). - H. Stichel, Gen. Ins., Fasc. 20, p. 15 (1904). - Id., Tierreich, Lief. 25, p. 48 (1909). - Fruhstorfer: A. Seitz, Großschmett. Erde, v. 5, p. 330 (1912). - H. Stichel, Neue Beitr. syst. Ins.kunde, v. 1, p. 7 (1916).

pannieulus Stich. $N$. p., H. Stichel, Ins.-Börse, v. Paraguay, 21, p. 6 (1904).

subsp. pannic ul us Stich. N. p., H. Sti-
chel, ut antea (1904). - Id., Gen. Ins., Fasc. 20, p. 15, t. 4, f. 2 (1904). - Id., Mato Grosso Tierreich, Lief. 25, p. 49 (distrib. part.) 
(1909). - Fruhstorfer, Ent. Zeit., v. 24, p. 194 (1910). - Id.: A. Seitz, Großschmett. Erde, v. 5, p. 330 (1912). - H. Stichel, Neue Beitr. syst. Ins.kunde, v. 1, p. 8 (1916).

subsp. pic cata Stich. N. p. piccatus, H. Bolivia

Stichel, Neue Beitr. syst. Ins.kunde, v. 1, p. (Rio Juntas) 8 (1916).

pusilla Röb. N. p., Röber, Int. Ent. Zeit., v. 23, p, Bolivia 325 (nov. gen. Naropina?) (1929).

sutor Stich. N. s., II. Stichel, Neue Beitr. syst. Ins.- Mato Grosso, kunde, v. 1, p. 7 (1916).

pl u to Tessm. N. p., Tessmann, Mitteil. Zool.

Süd-Peru

Mus. Berlin, v. 24, t. 5, f. 23 (1928).

\section{Sectio Peracurii}

H. Stichel, Gen. Ins., Fasc. 20, p. 16 (1904). - Id., Tierreich, Lief. 25 , p. 49 (1909).

albopunetum Stich. N. a., H. Stichel, Ins.-Börse, Peru v. 21, p. 6 (1904). - Id., Gen. Ins., Fasc. (Marcapata) 20 , p. 16 (1909). - Id., Tierreich, Lief. 25, p. 50, f. 13 (1909). - Fruhstorfer: A. 9 Seitz, Großsclimett. Erde, v. 5, p. 330 (1912).

\section{Tribus Caligonidi)}

H. Stichel, Neue Beitr. syst. Ins.kunde, v. 3, p. 59 (1925). Brassolinae (Subfam.) (part.) + Caligoninae (Subfam.) (part.), Fruhstorfer: A. Seitz, Großschmett. Erde, v. 5, p. 286, 290 (1912). - Caligonidae (Fam.) (part.), W. Rothschild, Nov. Zool., v. 23, p. 309 (1916).

\section{Genus Dynasfor E. Doubl.}

Dynastor (Boisduval MS), E. Doubleday (\& Westwood), Gen. Diurn. Lep., t. 58 (1849): J. O. Westwood, l. c., v. 2, p. 346 (Megastes Boisd. MS) (1851). - Ohenu \& H. Lucas, Enc. Hist. Nat., Pap. Diurn., p. 170 (1851-53). - Wallace, Trans. Ent. Soc. London, n. ser., v. 2, p. 261 (1854). - Ménétriés, IInum. Corp. Anim. Ac. Petropol. Lep., pars 1, p. 3s; pars 2, p. 77 (1855 \& 57). - Carus-Gerstäcker, Handb. Zool., v. 2, p. 232 (1863). - HerrichSchäffer, Corr.-Bl. Zool.-Min. Ver. Regensb., v. 18, p. 99 (1864). H. W. Bates, Journ. Entom., v. 2, p. 176 (1864). - HerrichSchäffer, ut antea, v. 19, p. 64 (1865). - Prittwitz, Stett. Ent. Zoit., v. 26, p. 310 (1865). - A. G. Butler, Cat. Diurn. Jep. Fabr., p. 40 (1869). - W. F. Kirby, Cat. Diurn. Lep., p. 127. (1871): Burmeister, Rev. Mag. Zool., ser. 3, v. 1, p. 19, 43, 45 (Dinastor!) (1873). - A. G. Butler \& Herb. Druce, Proc. Zool. Soc. London, p. 339 (1874). - S. II. Scudder, Histor. Sketch (Proc. Amer. Ac. Sci., v. 10), p. 160 (1875). - Wallace, Geogr. Distrib. Anim., v:i 2, p. 472 (1876). - Möschler, Verh. Zool.-Bot. Ges. Wien, v. 26, Abh., p. 321 (1877). - W. F. Kirby, Entomologist, v. 11, p. 26 (1878). - F. D. Godman \& O. Salvin, Biol. Centr.-Amer., Irep.Rhop., v. 1, p. 122 (1881). - C. Crüger, Berlin. Ent. Zeitą v. 25, p. 111 (1881). - E. Gerhard, l. c., v. 27, p. 179 (distrib.) (1883).

*) Rectius: Caliginidae.

Lepidopterorum Catalogus 51. 
- G. W. Müller, Zool. Jahrb. Syst., v. 1, p. 605, 633 (1886). - O. Staudinger (\& Schatz), Exot. Schmett., v. 1, p. 214 (1887). - L. Glaser, Cat. Etym. Col. Lep., p. 287, 365 (1887). - Röber: O. Staudinger \& Schatz, Exot. Schmett., v. 2, t. 32 (Morphol.), p. 191, 192 (1888 \& 89). - Richelmann, Tagebl. Deutsch. Naturf. Versamml., 61, p. 68 (1889). - W. F. Kirby, Jac. Hübner \& Geyer; Samml. Exot. Sehmett., neue Ausg., v. 3, p. 51 (sine synon.) (1901). $\rightarrow$ H. Stichel, Gen. Ins., Fasc., 20, p. 8, t. 1, f. 2 (Morphol.) (1904). Id., Tierrcich, Lief. 25, p. 20, f. 6 (Morphol.) (1909). - A. $\mathrm{Pa}$ genstecher, Geogr. 'Verbr. Schmett., p. 413 (1909). - Frulstorif'cr: A. Seitz, Großschmett. Erde, v. 5, p. 288 (1912). - W. Rothschild, Nov, Zool., v. 23, p. 309 (1916). - Le Cerf', Ene. Entom., ser. B 3, Lep., จ. 1, p. 141 (Anatom.) (1926). - Holdhaus: Chr. Schröder, Handb. Entom., v. 2, p. 731 (distrib. geogr.) (1927).

Danaus [Subgen.] (part.) Goeze, Ent. Beytr., v. 3, I, p. 124, 202 etc. (1779): - D. Festivus (part.) [D. H. Schneidcr], Nomencl. Entom., p. 35 (1785). - D. (part.), Gmelin-Linné, Syst. Nat., ed. 13, v. 1. V, p. 2259, 2279 $(1790)$.

"P ot a mis s uperba", Jac. Ḧ̈̈bncr, Samml. Exot. Schmett., v. 1, t. [72] (1806-19). - „P. S.", W. F. Kirby, I. c., Neue Ausg., t. 72 (1894).

Bras solis (part.), Jac. Hübner, Vorz. Schmett., 50 [1818]. - Id., Index Exot. Lep., p. 1 (1821). - Chonu \& H. Lucas, Enc. Hist. Nat., Pap. Diurn., p. 172 (1851-53). Herrich-Schäffer, Corr.-BI. Zool.-Nín. Ver. Regensb., V. 19, p. 64 (1865). - W. F. Kirby, Entomologist, v. 11, p. 26 (part.) (1878). - Id., Cat. Coll. Dium. Lep. Hewitson, p. 107 (1879).

Morpho (part.), Latreille (\& Godart), Eno. Méth., v. 9, p. 435 (1824). - J. B. Godart, 1. o., p. 452 etc. (1824).

Pa vonia (part.), J. B. Godart, ut antea, p. 807 (1824). - Verloren, Cat. Ins. Lep. Cramer, v. 2, p. 202 (1837). E. Doubleday, List Lep. Brit. Mus., v. 1, p. 118 (1844).

Megastes (part.), Boisduval, Consid. Lép. Guatemala, p. 53 (1870). - S. H. Scudder, Histor. Sketch (Proc. Amer. Ac. Sci., v. 10), p. 213 (1875).

- darius (Fabr.) Papilio d., J. C. Fabricius, Syst. Ent., Mittel- u. p. 482 (1775). - Dynastor d., H. Stichel, SüdGen. Ins., Fasc. 20, p. 9 (1904). - Id., Amerika Tierreich, Lief. 25, p. 22 (1909). - Fruh- bis storfer: A. Seitz, Großschmett. Erde, v. 5, Argentinien, p. 288 (1912).

Paraguay,

Trinidad

subsp. darius (Fabr.) Papilio d., J. C. Nord-, Fabricius, ut antea (1775). - P. (Danaus) Mitteld., Goeze, Ent. Beytr., v. 3. I, p. 202 (1779). Brasilien - P. d., J. O. Fabricius, Spec. Ins., v. 2, p. 57 (1781). - P. (Danaus Festivus) d., [D. H. Schneider], Nomencl. Entom., p. 35 (1785). - P. d., J. O. Fabricius, Mant. Ins., v. 2, p. 27 (1887). - P. (Danaus) d., Gmelin-I.inné, Syst. Nat., ed. $13, v, 1 . V_{.}$p. $2279(1790)$. - P. d., Jung, Alphab. Verz. Schmett., v. 1, p. 160 (1791). - J. C. Fabricius, Ent. syst., v. 3. I, p. 52; Index alphab., p. 121 (1793 \& 96). - Turton, bis Santa Catharina, NordArgentinien 
Gen. Syst. Nat., v. 3. II, p. 60 (1806). Morpho d., Pavonia d., (Latreille \&) J. B. Godart, Enc. Méth., v. 9, p. 452; 807 (1824). - Pavonia d., E. Doubleday, List Lep. Brit. Mus., v. 1, p. 118 (1844). - Dynastor d., (E. Doubleday \&) J. B. Westwood, Gen. Diurn. Lep., จ. 2, p. 347 (1851). Chenu \& II. Lucas, Enc. Hist. Nat. Pap. Diurn., p. 171 (1851-53). - Herrich-Schäffer, Corr.-Bl. Zool-Min. Ver. Regensb., v. 19, p. 64 (1865). - Prittwitz, Stettin. Ent. Zoit., v. 26, p. 310 (1865). - A. G. Butler, Cat. Diurn, Lep. Fabr., p. 40 (1869). - W. F. Kirby, Cat. Diurn. Lep., p. 127 (1871). - Burmeister, Rev. Mag. Zool., v. 1, p. 44, t. 6, f. 3 (Mletam.) (1873). W. F. Kirby, Entomologist, v. 11, p. 27 (1878). - Id., Cat. Coll. Diurn. Lep. Hewitson, p. 108 (1879). - P. H. Grosse, Entomol., v. 13 , p. 201 (1880). - F. D. Godman \& O. Salvin, Biol. Centr.-Amer., Lep.Rhop., v. 1, p. 123 (part. in cit.) (1881). W. Müller, Zool. Jalurb. Syst., v. 1, p. 595 (1886). - L. Glaser, Cat. Etymol. Col. Lep., p. 287, 365 (1887). - O. Staudinger (\& Schatz), Exot. Schmett., v. 1, t. 72; p. 214 (1886 \& 87). - Röber, 1. c., v. 2, t. 32 (Morphol.); p. 192 (1883 \& 89). Weymer, Ent. Zeit. Stettin, v. 55, p. 322 (1894). - Bönninghausen, Verh. Ver. Naturw. Unterh. Hamburg, v. 9 (1894-95), p. 38 (1896). - Brunner, Farbenpr. Ins., p. 6, t. 5, f. 54 (1897). - W. F. Kirby, Jac. Hübner \& Geyer, Samml. Exot. Scbmett., Neue Ausg., v. 3, p. 52 (cit. part.) (1901). - D. d. d., H. Stichel, Gen. Ins., Fase. 20, p. 9 (part.) (1904). - Id., Tierreich, Lief. 25 , p. 23 (forma principalis) (part.) (1909). - Fruhstorfer: A. Seitz, Großschmett. Erde, v. 5, p. 289 (1912). - D. d., W. Rothschild, Nov. Zool., v. 23, p. 319 , t. 6, f. 11,13 (larva, chrysal.) (1916). - P. Köhler, Fauna Argent., pars 1 (Zeit. wiss. Ins.-Biol., v. 18, Beiheft), p. 21 (1923). - D. d. d., H. Stichel, Neue Beitr. syst. Ins.kunde, v. 3, p. 62, 63 (1925). - D. d., Zikán, Ent. Rundschau, v. 45, p. 10 (1928).

forma $i c t$ erica Stich. D. d. icterinus, H. Typ.:

Stichel, Ins.-Börse, v. 21, p. 21 (1904). - Rio de D. $d$. $d$., forma $i .$, id., Gen. Ins., Fasc. 20, p. Janeiro 9 (1904). - D. d. d., forma icterica, id., Tierreich, Lief. 25, p. 24 (1909). - D. d. i., Fruhstorfer: A. Seitz, Großschmett. Erde, v. 5 , p. 289 (1912). - D. d. forma i., $H$. Stichel, Neue Beitr. syst. Ins.kunde, v. 3, p. 63 (1925).

subsp. a naxarete (Cram.) „Anaxarete", Guayana Papilio a., P. Cramer, Pap. Exot., v. 1, p. (Surinam) 148 , t. 95 , f. A, B; p. $151\left(\sigma^{\prime}\right)(1776)$. - ? Trinidad 
,Anaxareta", P. anaxarete, C. Stoll: l. c., v. 4, p. 167 , t. 374 , f. A, B (Q) ; p. 247 (1782). - P. (Danaus) anaxarete Goeze, Entom. Beytr., v. 3. I, p. 213 (1779). $P$. a., O. Stoll, Essai syst. Irep. Cramer, Pap. Exot., v. 4, App., p. 8, Nr. 23 (1782). - P. (Danaus Festivus) a., [D. H. Schneider], Nomencl. Entom., p. 35 (1785). $-P$. a., Jung, Alphab. Verz. Schmott., v. 1, p. 33 (1791). - $P$. anaxarethus, (Jablonsky \&) Herbst, Naturs. Ins., Schmett., v. 6, p. 65, t. 131 , f. $1-4$ (1793). - „Potamis superba anaxarete, Jac. Hübner, Samml. Exot. Schmett., v. 1, t. [72], i f. 3, 4 (1806-19). 'Brassolis a., id., Verz. Schmett., p. 50 (1818). - Id., Index Exot. Lep., p. 1 (1821). Morpho a., Pavonia a., (Latreille \&) J. B. Godart, Enc. Méth., v. 9, p. 452, 807 (1824). - P. a., Verloren, Cat. Ins. Lep. Cramer', v. 1, p. 47; v. 2, p. 202 (1837). - Dynastor a., Ménétriés, Enum. Corp. Anim. Ac. Petropol., Lep., pars 1, p. 38 (1855). "Potamis Superba a.", W. F. Kirby, Hübner \& Geyer, Samml. Exot. Schmett., Neue Ausg., v. 1, t. 72 (1894). - Dynastor darius a., H. Stichel, Neue Beitr., syst. Ins.kunde, v. 3, p. 63 (1925).

D. darius, Möschler, Verh. Zool.-Bot. Ges. Wien, v. 26, Abh., p. 321 (1877). - ? W. J. Kaye, Trans. Ent. Soc. London, p. 166 (1904). - D. d. d., H. Stichel, Gen. Ins., Fasc. 20, p. 9 (part. in cit.) (1904). - Id., Tierreich, Lief. 25, p. 23 (part. in cit.) (1909).

forma populus Röb. D. p., Röber, Int. Typ.: Franz.Ent. Zoit., v. 21, p. 141, t., f. 1 (1927). Guayana

subsp. faenius Fruhst. D. d. f., Fruhstor- Rio Grande fer: A. Seitz, Großschmett. Erde, v. 5, p. do Sul 289 (1912). - D. d. taenius (!), H. Stichel, Neve Beitr. syst. Ins.kunde, r. 3, p. 63 (nom. vix conserv.) (1925).

D. darius, Woymer, Stettin. Ent. Zoit., v. 55 (1894), p. 322 (1895).

subsp. ma $\eta^{\circ}$ on $i$ s F Fruhst. D. d. m., Fruh- Paraguay, storfer: A. Scitz, Großschmott. Erde, v. 5, ? Argentinien t. 62 b; p. 289 (1911 \& 12). - ? D. d. forma m., P. Köhler, Fauna Argent., pars 1 (Zeit. (Misiones) wiss. Ins.-Biol., v. 18, Beiheft), p. 21 (1923). - D. d. mardonus (!), H. Stichel, Neuo Beitr. syst. Ins.kunde, v. 3, p. 63 (1925). - D. d. mardonius, C. B. Williams, Migrat. Butterfl., p. 241 (1930).

subsp. styg ia n u s Butl. D. s., A. G. Butler, GuatemalaCistula Ent., v. 1, p. 73 (1S72). - Id., Lep. Colombia, Exot., p. 125, t. 47 , f. 1 (1873). - A. G. ? Ecuador, Butler \& Herb. Druce, Proc. Zool. Soc. Bolivia London, p. 339 (1874). - W. F. Kirby, Cat. Diurn. Lep., Suppl., p. 716 (1877). - D. 
darius s., H. Stichel, Gen. Ins., Fase. 20, p. 9, t. 3, f. 2 (1904). - Id., Tierreich, Lief. 25, p. 24 (1909). - Fruhstorfer: A. Seitz, Großschmett. Erde, v. 5, p. 288 (1912). H. Stichel, Neue Beitr. syst. Ins.kunde, v. 3, p. 63 (1925).

Morpho anaxarete (non Pap. a. Cramer), Kollar, Denkschr. Ak. Wissensch. Math. Naturw. Cl., v. 1, p. 351 (1849).

Megastes darius, Boisluval, Consid. Lép. Guatemala, p. 53 (1870). - Dynastor d., F. D. Godinan \& O. Salvin, Biol. Centr.-Amer., Lep.-Rhop., v. 1, p. 123 (part.) (1881).

macrosiris (Doubl.) Brassolis $m$. (Boisduval MIS), FranzösischE. Doubleday (\& Westwood), Gen. Diurn. Guayana, Lep., t. 59, f. 3 (1849). - Dynastor m., H. Trinidad, Stichel, Gen. Ins., Fasc. 20, p. 10 (1904). - VenezuelaId., Tierreich, Lief. 25, p. 25 (1909). - Mexiko, Fruhstorfer: A. Seitz, Großschmett. Erde, Bolivia v. 5 , p. 289 (1912).

subsp. macrosiris (Doubl.) Brassolis m., Franz.E. Doubleday (\& Westwood), ut antea Guayana (1849); J. O. Westwood, 1. c., v. 2, p. 351 (Cayenne), (1851). - Chenu \& H. Lucas, Enc. Hist. Trinidad Nat., Pap. Diurn., p. 173 (1851-53). Herrich-Schäffer, Corr.-BI. Zool.-Min. Ver. Regensb., v. 19, p. 64 (1865). - W. F. Kirby, Cat. Dium. Lop., p. 125 (1871). - Id., Entomologist, v. 11, p. 26 (1878). - Opsiphrnes m., id., Cat. Coll. Diurn. Lep. Hewitson, p. 107 (1879). - Dynastor m., O. Staudinger (\& Schatz), Exot. Schmett., v. 1, p. 214 (1887). - Röber, 1. c., v. 2, p. 192 (1889). - I. Stichel, Berlin. Ent. Zeit., v. 46 , p. 523 (1902). - D. m. m., id., Gen. Ins., Fasc. 30, p. 10 (1904). - Id., Tierreich, Lief. 25, p. 25 (1909). - Fruhstorfer: A. Seitz, Großschmett. Erde, v. 5, p. 289 (1912). - D. m., W. J. Kaye, Trans. Ent. Soc. London, p. 549 (distrib. part.) (1914).

subsp. strix (Bat.) Brassolis s., A. G. But- Mexiko, ler, Ent. Monthly Mag., v. 1, p. 164 (1864). Guatemala, - Herrich-Schäffer, Corr.-Bl. Zool.-Min. Ver. Venezuela Regensb., .19, p. 64 (1865). - W. F. Kirby, Cat. Diurn. Lep., p. 125 (1871). - Dymastor s., F. D. Godman \& O. Salvin, Biol. Centr.-Amer., Lep.-Rhop., v. 1, p. 123, t. 12, f. 3, 4 (Brassolis s.) (1881). - O. Staudinger (\& Schatz), Exot. Schmett., v. 1, p. 214 (1887). - Röber, l. c., v. 2, p. 192 (1889). - H. Stichel, Berlin. Ent. Zeit., v. 46, p. 523 (1902). D. macrosiris s., H. Stichel, Gen. Ins., Fasc. 20, p. 10 (cit. part.) (1904). - Id., Tierreich, Lief. 25, p. 26 (cit. part.) (1909). Fruhstorfer: A. Seitz, Großschmett. Erde, v. 5 , t. 62 b; p. 289 (1911 \& 12). - W. Rothschild, Nov. Zool., v. 23, p. 310 (1916). 
subsp. $h a n n i b a l$ Oberth. $D$. $h$., Ch. Ober- Colombia thür, Etudes Ent., v. 6, p. 28 (1881). - D. macrosiris h., Fruhstorfer: A. Seitz, Großschmett. Erde, v. 5, p. 289 (1912). - W. Rothschild, Nov. Zool., v. 23, p. 310 (1916).

D. macrosiris strix, H. Stichel, ut antea (part. in cit.) (1904 \& 09).

subsp. $p h a r n a c e s$ Stich. D. m. p., H. Sti- Bolivia chel, Berlin. Ent. Zeit., v. 52 (1907), p. 174 (1908). - Id., Tierrcich, Lief. 25, p. 26 (1909). - Fruhstorfer: A. Seitz, Großschmett. Erde, v. 5, p. 290 (1912). - D. m. pharnacer (!), D. Sharp, Zool. Record, v. 45 (1908), pars 12, p. 316 (1910).

- Napoleon Doubl. D. n. (Boisduval MS), E. Double- Rio de day (\& Westwood), Gen. Diurn. Lep., t. 58, f. $2(1849) ; D$. napoleo (!), J. O. Westwood, l. c., v. 2, p. $347(1851)$. - D. napoleon, Herrich-Schäffer, Außereur. Schmett., t. 19, f. 79,80 ; p. 55 (falso f. 89,90$)(1850 \& 58)$ (conf. Dalla Torre, Entom. Nachr. Bl., Troppau, v. 1, 1927, p. 5, 73: Dynaror (!) n.). Dynastor napoleo, D. napoleon, Chenu \& H. Lucas, Enc. Hist. Nat., Pap. Diurn., p. 171, t. $33(1851-53)$. $-D$. napoleon, Ménétriés, Enum. Corp. Anim. Ac. Petrop., Lep., pars 2, p. 77 (1857). - Herrich-Schäffer, Corr.B1. Zool.-Min. Ver. Regensb., v. 19, p. 64 (1865). - W. F. Kirby, Cat. Diurn. Lep., p. 127 (1871). - Burmeister, Rev. Mag. Zool., ser. 3, v. 1, p. 44 (not. biol.) (1873). - W. F. Kirby, Entomologist, v. 11, p. 26 (1878). - Id., Cat. Coll. Diurn. Lep. Hewitson, p. $10 \mathrm{~S}(1879)$. I. Glaser, Cat. Etymol. Col. Lep., p. 287 (1887). O. Staudinger (\& Schatz), Exot. Schmett., v. 1 , t. 73 ; p. 214 (1886 \& 87). - Röber, l. c., v. 2, t. 32 (Morphol.); p. 192 (1888 \& 89). - W. F. Kirby, Handb. Lep., v. 1, p. 199 (1894). - Bönninghausen, Verh. Ver. Naturw. Unterh. Hamburg, v. 9 (1894-95), p. 38 (not. biol.) (1896). - H. Stichel, Gen. Ins., Fasc. 20, p. 10 (1904). - Id., Tierreich, Lief. 25, p. 27 (1909). - H. Fruhstorfer: A. Seitz, Großschmett. Erde, v. 5, t. 62b; p. $290(1911 \& 12)$. - W. J. Kaye, Proc. South London Ent. Nat. Hist. Soc. $(1910-11)$, p. 5 (1911). - W. Rothschild, Nov. Zool., v. 23 , p. 309, 310, t. 3, f. 5, 6 (larva, chrysal.) (1916). - Zikán, Biol. Beitr.: Zeitschr. Deutsch. Vcr. Wissensch. Kunst, Sao Paulo, v. 1 (Thering Festschr.), p. 150, 2 fig., t. 12 (Biol.). - Id., Ent. Rundschau, v. 45, p. 10 (1928).

forma maculat a Niep. $D$. $n$. forma macr- Typ.: Rio latus, Niepelt, Int. Ent. Zeit., v. 16, p. 68 de Janeiro

Janeiro,

Santa

Catharina

(Itatiaya) (1922). 


\section{Genus Dasyophthalma J. O. Westw.}

(E. Doubleday \&) J. O. Westwood, Gen. Diurn. Lep., v. 2, p. 343 (1851). - Chenu \& H. Lucas, Enc. Hist. Nat., Pap. Diurn., p. 168 (1851-53). - Ménétriés, Enum. Corp. Anim. Ac. Petropol., Lep., pars 1, p. $396(1855)$. - H. W. Bates, Journ. Ent., v. 2, p. 176 (1864). - Herrich-Schäffer, Corr.-Bl. Zool.-Min. Ver. Regensb., v. 18, p. 99 ; v. 19 , p. 65 (1864 \& 65). Prittwitz, Stettin. Int. Zeit., v. 26, p. 309 (1865). - W. F. Kirby, Cat. Diurn. Lep., p. 129 (1871). - A. G. Butler, Lep. Exot., p. 125 (1873). - S. H. Scudder, Iist. Sketch: Proc. Amer. Ac. Sci., v. 10, p. 155 (1875). - Dusyopthalma (!), Wallace, Geogr. Distr. Anim., v. 2, p. 472 (1876). - Dasyophthalma, F. Müller, Jenaisch. Zeit. Naturw., v. 11, p. 102 (Molphol. Physiol.) (1877). - Id., Kosmos, Leipzig, v. 1, p. 392, 394 (1877). - Id., Trans. Ent. Soc. London, p. 214 (odor) (1878). - W. F. Kirby, Entomologist, v. 11, p. 28 (1878). - E. Gerhard, Berlin. Ent. Zoit., v. 27, p. 179 (distrib. geogri.) (1883). - Dalla Torre (sec. F. Müller), hosmos, Leipzig, v. 17, p. 411 (1885). - I. Glaser, Cat. Etymol. Col. Lep., p. 285 (1887). - O. Staudinger (\& Schatz), Exot. Schmett., v. 1, p. 217 (1887). Röber, 1. c., v. 2, t. 32 (Morphol.); p. 191, 194 (1888 \& 89). E. Reutcr, Acta Soc. Sci. Fenn., v. 22, p. 114, 553 (1896). - W. F. Kirby, Hübner \& Geyer, Samml. Exot. Schmett., Neue Ausg., v. 3, p. 53 (1901). - H. Stichel, Gen. Ins., Fasc. 20, p. 1.0, t. 1, f. 3 (Morphol.) (1904). - Silva, Relat. Contrib. IIist. Nat. Lep. Brasil (Congr. Scieut. Lat.-Amer., 1905, v. 3B) p. 98 (1907). - H: Stichel, Tierreich, Lief. 25, p. 29, f. 7 (Morphol.) (1909). - A. Pagenstechor, Geog1. Verbr. Schmett., p. 413 (1909). - Fruhstorfer: A. Seitz, Großschmett. Erde, v. 5, p. 290 (1912). - Dasiophtalma (!), P. Köhler, Fauna Argent., pars 1 (Zeit. wiss. Ins.-Biol., v. 18, Beiheft), p. 21 (1923). - Le Cerf, Enc. Ent., ser. B 3, Lep., v. 1, p. 141 (Anatom.) (1926). - Holdhaus: Chr. Schröder, Handb. Entom., v. 2, p. 731 (distrib. geogr.) (1927).

„P o t a mis s u per $b a^{\prime \prime}$, Jac. Hübner, Samml. Exot. Schmett., v. 1 , t. (72) (1806-19). - P. (part.), id., Cat. Lép. Franck, p. 79 (1826).

C a l ig o, Jac. Hübner, Samml. Exot. Schmett., v. 2, t. 68, $69(1820-26)$. - E. Doubleday, List Lep. Brit. Mus., pars 1 , p. 117 (part.) (1844).

Morpho (part.), Latreille (\& Godart), Enc. Méth., v. 9, p. 435 ; J. B. Godart, 1. c., p. 451 etc. (1824). - L. Glaser, Cat. Etymol. Col. Lep., p. 364 (1887).

Pavonia, (Latreille \&) J. B. Godart, Enc. Méth., v. 9, p. 807 (1824). - II. Lucas, Hist. Nat. Lép.-Exot., p. 141 (1835). - E. Blanchard, Hist. An. Artic., v. 3, p. 454 (1840). - E. Blanchard (\& Doyère): Cuvier, Règne Anim., ed. 4 (Masson-Crochard), v. 2, Ins., p. 246 (1849). L[ucas]: Orbigny, Dict. Hist. Nat., Atl., v. 2, p. 18 (1849). - E. Doubleday (\& Westwood), Gen. Diurn. Lep., t. 56 (1849).

Opsiphanes (part.), W. F. Kirby, Cat. Coll. Diurn. Lop. Hewitson, p. 107 (1879).

- rusina (God.) Morpho r., Pavonia r., (Latreille Mittel-,

\&) J. B. Godart, Énc. Méth., v. 9, p. 451, Süd807 (1824). - Dasyophthalma r., H. Sti- Brasilien chel, Gen. Ins., Fasc. 20, p. 11 (1904). Id., Tierreich, Lief. 25, p. 30 (1909). Fruhstorfer: A. Seitz, Großschmett. Erde, v. 5, p. 291 (1912). 
subsp. rusina (God.) Morpho r., Pavonia r., Santa J. B. Godart, ut antea (1824). - Caligo r., Catliarina, E. Doubleday, List Lep. Brit. Mus., v. 1, p. Rio de 117 (1844). - Pavonia r., E. Doubleday. (\& Janeiro Westwood), Gen. Diurn. Lep., t. $56, \mathrm{f} .1$ (1849); Dasyophthalma r., J. O. Westwood, 1. c., v. 2, p. 344 (1851). - D. r., Chenu \& H. Lucas, Enc. Hist. Nat., Pap. Diurn., p. 169, f. 280 (1851-53). - Ménétriés, Enum. Corp. Anim. Ac. Petropol., pars 1, p. 38 (1855). - Herrich-Schäffer, Corr.-B1. Zool. Min. Ver. Regensb., v. 19, p. 65 (1865). W. F. Kirby, Cat. Diurn. Lep., p. 129 (1871). - Id., Entomologist, v. 11, p. 28 (1878). - Opsiphanes r., id., Cat. Coll. Diurn. Lep. Hewitson, p. 107 (1879). - Dasyophthalma r., O. Staudinger (\& Schatz), Exot. Schmett., v. 1, t. 76 ; p. 217 (1886 \& 87). - Röber, 1. c., v. 2, p. 194 (1889). L. Glaser, Cat. Etymol. Col. Lep., p. 285 (1887). - Bönninghausen, Verh. Ver. Naturw. Unterh. Hamburg, v. 9 (1894-95), p. 38 (1896). - D. r. r., H. Stichel, ut antea (1901 \& 1909). - Fruhstorfer, ut antea (1912). - Zikán, Ent. Rundschau, v. 45, p. 10 (1928).

lyca on (Lucas) Pavonia $l$., H. Lucas, Hist. Nat. Tép. Exot,, p. 141, t. 78, f. 1 (1835).

del anir a Hew. Dasyophthalma d., Hewitson, Exot. Butt., v. 4 (3), t. [3] Dasyophthalma \& Thaumantis, f. 1 (\%) (1862). - HerrichSchäffer, Corr.-B1. Zool.-Nin. Ver. Regensburg, v. 19 , p. 95 (1865). - Opsiphanes $d$. ., W. F. Kirby, Cat. Coll. Diurn. Lep. Hewitson, R. 107 (1879).

forma Donckier $i$ Joic. \& Talb. D. rusina Typ.: ? ab. $d$., Joicey \& Talbot, Bull. Hill Mus., v. 1, p. 357 (क) (1922).

subsp. $p r i n c i p e s a$ Stich. D. $r$. p. (D. $p$. Espirito Frulistorfer MSS), H. Stichel, Ins.-Börse, Santo v. 21, p. 21 (1904). - Id., Gen. Ins., Fase. 20, p. 11 (1904). - Id., Berlin. Ent. Zeit., v. 52 (1907), p. 178 (1908). - Id., Tierreich, Lief. 25, p. 31 (1909). - Fruhstorfer: A. Seitz, Großschmett. Ende, v. 5, t. 62 a; p. 291 (1911 \& 12).

subsp. geraensis Reb. D. r. g., Rebel, Süd-Minas Verh. Zool.-Bot. Ges. Wien, v. 71 (1821), Geraes, p. (14) (1922). - D. g., Zikán, Ent. Rund- Santa schau, v. 45 , p. 10 (1928).

Catharina

(Itatiaya)

- creusa (Hübn.) Caligo c., Jac. Hübner, Samml. Mittel-, Exot. Schmett., v. 2, t. [68] (1822-26). - SüdDasyophthatma c., H. Stichel, Gen. Ins., Brasilien, Fasc. 20, p. 11 (1904). - Id., Tierreich, ? Bahia, Lief. 25, p. 32 (1909). - Fruhstorfer: A. Pará Seitz, Großschmett. Erde, v. 5, p. 290 (1912). 
subsp. creusa (Hübn.) Caligo c., Jac. Santa Hübner, ut antea, t. [68], f. 1, 2( $\left.\sigma^{7}\right)$; t. [69], Catharina f. 3, 4 (O) (1822-26). - Potamis c., id., Cat. Coll. Lép. Tranck, p. 79 (1826). Caligo c., E. Doubleday, List Lep. Brit. Mus., v. 1, p. 117 (1844). - Dasyophthalma c., (E. Doubleday \&) J. O. Westwood, Gen. Diurn. Lep., v. 2, p. 344 (1851). Chenu \& II. Lueas, Enc. Hist. Nat., Pap. Diurn., p. 169 (1851-53). - Herrich-Schäffer, Corr.-Bl. Zool.-Min. Ver. Regensb., v. 19, p. 65 (1865). - Prittwitz, Ent. Zeit. Stettin, v. 26, p. 309 (not. biol.) (1865). W. F. Kirby, Cat. Diurn. Lep., p. 129 (1871). - Td., Entomologist, v. 11, p. 28 (1878). - Opsiphanes c., id., Cat. Coll. Diurn. Lep. Hewitson, p. 107 (1879). - Chr. Aurivillius, Svenska Ak. Handl., v. 19, Nr. 5, p. 74 (1882). - O. Staudinger (\& Schatz), Exot. Schmett., v. 1, t. 76 ; p. 218 (1886 \& 87). - Röber, 1. c., v. 2, t. 32 (Morphol.); p. 194 (1888 \& 89). - L. Glaser, Cat. Etymol. Col. Lep., p. 285 (1887). - A. Seitz, Ent. Zeit. Stettin, v. 51, p. 29 (not. biol.) (1890). - Catigo c., W. F. Kirby (sec. IIübner), Samml. Exot. Schmett. Hübner \& Geyer, Neu $\ominus$ Ausg., v. 2, t. 2s1, 82 (68, 69) (1898). - Dasyophthalma c., id., ibid., v. 3, p. 53 (1901). - D. c. c., H. Stichel, ut antea (1904 \& 09). - D. c., Silva, Relat. Contrib. Hist. Nat. Lep. Brasil (Congr. Scient. Lat.-Amer., 1905, v. 3, B), p. 98 (1907). - D. c. c., Fruhstorfer, ut antea, t. 62 c (1912 \& 11). - Dasiophtalma (!) c., P. Köhler, Fauna Argent. pars 1 (Zeit. wiss. Ins.-Biol., v. 18, Beiheft) p. 21 (1923). - Dasyophthalma c., Zikén, Ent. Rundschau, v. 45 , p. 10 (1928). - D. brensa (err. typ.!), Loquai, Int. Ent. Zeit., v. 22, p. 157 (1928). a naxandra (God.) Morpho a., Pavonia a., (Latreille \&) J. B. Godart, Enc. Méth., v. 9 , p. 451,807 (Q) (1824). - P. a., E. Blanchard, Hist. An. Art., v. 3, p. 454, t. 17, f. 1 (1840). - P. a. (Boisduval MS), E. Blanchard (\& Doyère): Cuvier, Règne Anim., ad. 4 (Masson-Crochard), v. 2, Ins., p. 246, t. 141 , f. 1 (o), f. 1 a, b (caput, ant.) (1849). - H. L[ucas]: Orbigny, Dict. Hist. Nat., Atlas, v. 2, p. 18, t. 6, f. $2 \mathrm{a}, \mathrm{b}$ (1849).

Papilio sophorae (non Linné), E. Donovan, Natural. Repos., v. 3, t. 87, 88 (1825). subsp. ba rone $a$ S Stich. $D$. c. $b$., (D. $b$., Espirito Fruhstorfer MS), H. Stichel, Ins-Börse, Rio de v. 21 , p. 21 (1901). - Id., Gen. Ins., Fasc. Janeiro 20, p. 11 (1904). - Id., Berlin. Ent. Zeit., Santo, v. $52(1907)$, p. 178 (1908). - Id., Tierreich, Lief. 25, p. 34, f. 8 (1909). - Fruhstorfer: A. Seitz, Großschmett. Erde, v. 5, p. 290 (1912). 
D. cre $и$ sa, Bönninghausen, Verh. Ver. Naturw. Unterh. Hamburg, v. 9 (1894-95), p. 38 (1896).

vertebralis Butl. D. v., A. G. Butler, Cistula Ent., Espirito v. 1, p. 2 (1869). - W. F. Kirby, Cat. Diurn. Lep., p. 129; Suppl.,p. 717 (1871 \& 77). - A. G. Butler, Lep. Exot., p. 125, t. 47 , f. 2 (1873). - O. Staudinger (\& Schatz), Exot. Schmett., v. 1, p. 218 (1887). - H. Stichel, Gen. Ins., Fasc. 20, p. 11, t. 3, f. 3 (1904). - Id., Tierreich, Lief. 25, p. 35 (1909). - Fruhstorfer: A. Seitz, Großschmett. Erde, v. 5, t. 62 a, p. 291 (1911 2 \& 12).

\section{Genus Opsiphanes E. Doubl.}

E. Doubleday (\& Westwood), Gen. Diurn. Lep., t. 57 (1849); J. O. Westwood, ibid., v. 2, p. 344 (Meropis Boisduval MS) (part.) (1851). - Chenu \& H. Lucas, Inc. Hist. Nat., Pap. Diurn., p. 170 (185153). - Wallace, Trans. Ent. Soc. London, ת. вer., จ. 2, p. 261 (not. biol.) (1854). - Ménétriés, Enum. Corp. Anim. Ac. Petropol., pars 1, p. 38 (1855). - Carus-Gerstäcker, Handb. Zool., v. 2, p. 232 (1863). - Herrich-Schäffer, Corr.-B1. Zool--Min. Ver. Regensb., v. 18 , p. 99 ; v. 19 , p. 64 (part.) $(1864 \& 65)$. - H. W. Bates, Journ. Entom., v. 2, p. 176 (1864). - Prittwitz, Ent. Zeit. Stettin, v. 26, p. 309 (1865). - G. Koch, Indo-Austral. Lep.-Fauna, ed. 1, 2, p. 116 (1865 \& 73). - A. G. Butler, Cat. Diurn. Lep.: Fabr., p. 40 (part.) (1869). - W. F. Kirby, Cat. Diurn. Lep', $\rho$ 125; Suppl., p. 716 (part.) (1871 \& 77). - A. G. Butler \& Herb. Druce, Proc. Zool. Soc. London, p. 339 (1874). - A. G. Butler, Proc. Ent. Soc. London, p. 425 (1874). - S. H. Scudder, Hist!! Sketch: Proc. Amer. Ac. Sci., v. 10, p. 40 (1875). - Herb. Druce, Proc. Zool. Soc. Tondon, p. 217 (part.) (1876). - Wallace, Geogr. Distr. Anim., v. 2, p. 472 (1876). - Distant, Proc. Ent. Soc. London, p. XIII (1876). - Möschler, Verh. Zool.-Bot. Ges. Wien, v. 26, Abh., p. 321 (1877). - Fr. Müller, Jenaisch. Zeit. Naturw., v. 11, p. 31 (Morphol.) (1877). - A. G. Butler, Trans. Ent. Soc. London, p. 114 (1877). - Fr. Müller, Trans. Ent. Soc. London, p. 214 (odor) (1878). - Id., Ent. Zeit. Stettin, v. 39, p. 296 (1878). - W. F. Kirby, Entomologist, v. 11, p. 26 (part.) (1878). - Id., Cat. Coll. Diurn. Lep. Iewitson, p. 107 (1879). - F. D. Godman \& O. Salvin, Biol. Centr.-Amer., Lep.-Rhop., v. 1, p. 125 (part.) (1881). - C. Orüger (sec. Hewitson), Berlin. Ent. Zeit., v. 25, p. 111 (1881). - O. (Subgen.) (part.), Chr. Aurivillius, Svenska $A$ k. Handl., n. ser., v. 19, Nr. 5, p. 75 (1882) - O., E. Gerhard, Berlin. Ent. Zeit., v. 27, p. 179 (distrib. geogr.) (1883). G. T. Müller, Zool. Jahrb. Syst., v. 1, p. 605 (1886). -' O. Staudinger (\& Schatz), Exot. Schmett., v. 1, p. 211 (1887). - I. Glaser, Cat. Etymol. Col. Lep., p. 304 (part.) (1887). - Röber: 0. Staudinger \& Schatz, Exot. Schmett., v. 2, t. 32 (Morphol.), p. 191, 192 (1888 \& 89). - Mahnel, Deutsch. Fnt. Zeit. Lep. (Tris), v. 3, p. 166 etc., sec. Index. p. 329 (1890). - W. T.j Kirby, Handb. Lep., v. 1, p. 199 (1894), - E. Reuter, Acta Soc. Sci. Fenn., v. 22, p. 112, 553 (part.) (1896). - Opsifanes (!), Tristan, Ins. Costa Rica, p. 18 (1897). - Opsiphanes, W. F. Kirby, Hübner \& Geyer, Samml. Exot. Schmett., Neue Ausg., v. 3, p. 50 (cit. part) (1901). - 
F. Stichel, Berlin. Ent. Zeit., v. 46 (1901), p. 503 (1902). — Id, Gen. Ins., Fasc. 20, p. 16, t. 1, f. 6 (Morphol.) (1904). - Id., Soc. Entom., v. 22, p. 92 (1907). - Silva, Relat. Contrib. Hist. Nat. Lop. Brasil (Congr. Scient. Lat.-Amer., 1905, v. 3 B), p. 95 (party) (1907). - H. Stichel, Tierreich, Lief. 25, p. 51, f. 14 (Morphol.) (1909). - A. Pagonstecher, Geogr. Verbr. Sclimett., p. 413 (1909). - Fruhstorfer: A. Soitz, Großschmett. Erde, v. 5, p. 291 (part.); p. 293 (Artengruppe) (1912). - Stand, Lep. Niepeltiana, v. 2, p. 12 (1916). - Strand, Soc. Entom.x 33, p. 19 (1918). - Campos, Rev. Col. Nacion. Vicente Rocafuerte, Nr. 4, p. 31 (1921). - Jurriaanse, Tijdschr. Entom., v. 66, p. 147 (1923). - Schwanwitsch, Proc. Zool. Soc. London, p. 522 (Morphol.) (1924). - Handlirsch: Chr. Schröder, Handb. 'Entom., v. 3, p. 939 (1925). - Le Cerf', Enc. Entom., ser. B, 3, Lep., v. 1, p. 141 (Anatom.) (1926). Holdhaus: Chr. Schröder, Handb. Entom., v. 2, p. 743 (distrib. geogr.) (1027).

Da naus [Subgen.] (part.), Linné, Syst. Nat., ed. 10, p. 468 (1758). - Linné-Houttyn, Natuurl. Hist., v. 1, XI, p. 270 (1766-69). - Linné, Syst. Nat., od. 12, v. 1. II, p. 758, 767 (1767). - Goeze, Ent. Beytr., v. 3. I, p. 124, 193 etc. (1779). - D. (Festivus) (part.), [D. H. Schneider], Nomencl. Entom., p. 35 (1785). - D. (part.), Gmelin-Linné, Syst. Nat., ed. 13, v. 1. V, p. 2280 (1790).

$N y m p h a l i s$ (Gemmatus), [Subgen.] (part.), [D. H. Schneider], Nomencl. Entom., p. 37 (1785).

Brassolis (part.), Latreille, Gen. Crust. Ins., v. 4, p. 195 (1806-09). - J. C. Fabricius MS: Illiger, Mng. Ins.kunde, v. 6, p. 282 (1807). - Jac. Hübner, Verz. Schmett., p. 50 (1818). - Id., Index Exot. Lep., p. 2 (1821). - Id., Samml. Exot. Schmett., v. 2, t. 66 (1822-26). - J. G. Children (sec. Fabr.), Philos. Mag., v. 7, p. 120 (1830).

„P o tamis superba", Jac. Hübner, Samml. Exot. Schmett., v. 1, t. 74-76 [1806-19]. - „P. Superba“, W. F. Kirby (sec. Hübner), I. c., Neue Ausg., ut antea (1894).

Caligo, Jac. Hübner, Samml. Exot. Schmett., v. 2, t. 70, $71(1820-26)$. - E. Doubleday, List Lep. Brit. Mus., v. 1, p. 117 (part.) (1844). - Boisduval, Consid. Lép. Guatemala, p. 54 (part.) (1870). - Capronnier, Ann. Soc. Ent. Belg., v. 17, p. 28 (1874).

Morpho (part.), Latreille (\& Godart), Enc. Méth., v. 9, p. 435; J. B. Godart, 1. c., p. 454 etc. (1824)i-

Pavoonia (part.), (Latreille \&) J. B. Godart, Enc. Méth., v. 9, p. 807 (1824). - Verloren, Cat. Ins. Lep. Cramer, v. 2, p. 202 (1837). - E. Blanchard, Hist. An. Artic., v. 3. p. 454 (1840). - E. Doubleday, List Lep. Brit. Mus., v. 1, p. 118 (1844). - E. Blanchard, Hist. Ins., v. 2, p. 334 (1845). - L[ucas]: Orbigny, Dict. Hist. Nat., v. 9, p. 515 (1847). - P. (Caligo + Opsiphanes) (part.) Burmeister, Rev. Mag. Zool., ser. 3, v. 1, p. 31, 41 (1873). - Id, Descr. Phys. Rep. Argent., v. 5, pars 1, p. 199 (1878).

Blepolenis, Röber, Soc. Ent., v. 21, p. 18 (1906). - H. Stichel (sec. Röber), l. c., v. 22, p. 92 (1907).

\section{a. Cohors Bafeiformes}

H. Stichel, Gen. Ins., Fasc. 20, p. 17 (1904). - Id., Soc. Entom., v. 22, p. 92 (1907). - Id., Tierreich, Lief. 25, p. 53 (1909). 
- batea (Hübn.) Caligo b., Jac. Hübner, Samml. Exot. Espirito Santo Schmett., v. 2, t. 70 (1822-26). - Opsiphanes b., H. Stichel, Berlin. Ent. Zeit., v. 52 (1907), p. 171 (1908). - Id., Tierreich, Lief. 25, p. 54 (1909). - Fruhstorfer: A. Seitz, Großschmett. Erde, v. 5, p. 294 (part.) (1912). - H. Stichel, Neue Beitr. syst. Ins.kunde, v. 3, p. 63 (1925).

subsp. $b$ a t $e$ a (Hübn.) Caligo $b$., Jac. Hüb- Espirito Santo ner, ut antea, f. 1, 2 (1822-26). - E. Doubleday, List Lep. Brit. Mus., v. 1, p. 117 (1844). - Opsiphanes b., (E. Doubleday \&) J. O. Westrood, Gen. Diurn. Lep., v. 2, p. 345 (1851). - Herrich-Schäffer, Corr.B1. Zool.-Min. Ver. Regensb., v. 19, p. 64 (1865). - W. F. Kirby, Cat. Diurn. Lop., p. 125 (1871). - Pavonia b., Burmeister, Rev. Mag. Zool., ser. 3, v. 1, p. 43 (in cit. savonia God.) (1873). - Opsiphnnes b., W. F. Kirby, Entomologist, v. 11, p. 26 (1878). - Id., Cat. Coll. Diurn. Irep. ILewitson, p. 107 (1879). - Chr. Aurivillius, Svenska Ak, Hand., n. ser., v. 19, Nr. 5, p. 75 (1882). O. Staudinger (\& Schatz), Exot. Schmett., v. 1, p. 212 (1887). - Bönninghausen, Verh. Ver. Naturw. Unterh. Hamburg, v. 9 (189495), p. 38 (1896). - Caligo b., Opsiphanes Z., W. F. Kirby, Samml. Exot. Schmett. Hübner \& Geyer, Neue Ausg., v. 2, t. 283 (70), f. 1, 2; v. 3, p. 51 (1898 \& 1901). o. b., H. Stichel, Berlin. Ent. Zeit., v. 46, (1901), p. 504 (1902). - Id., Gen. Ins., Fasc. 20, p. 17 (1904). - o. b. b., id., Soc. Ent., v. 22, p. 98 (Q) (1907). - O. b. b., id., Berlin. Ent. Zeit., v. 52 (1907), p. 171 (1908). - Id., Tierreich, Liel. 25, p. 55 (1909). - Fruhstorfer: A. Seitz, Großschmett. Erde, v. 5, t. 62 c; p. 294 (1911 \& 12). - W. Rothschild, Nov. Zool., v. 23, p. 310 , t. 5, f. 8, 6 (larva, chrysal.) (1916). - Fruhstorfer, Ent. Rundschau, v. 34, p. 56 (1917). - H. Stichel, Neue Beitr. syst. Ins.kunde, v. 3, p. 64 (1925).

saronia (God.) Morpho s., Pavonia s., (Latreille) \& J. B. Godart, Enc. Méth., v. 9, p. 450, 807 (1824). - Opsiphanes s., Ménétriés, Fnum. Corp. Ac. Petrop., Lep., pars 1, p. 38 (1855).

forma $a s p h$ er $u s$ Fruhst. O. b. a., Fruh- Typ.: Espirito storfer: A. Seitz, Großsehmett. Erde, v. 5, Santo p. 294 (1912). - Id., Ent. Rundschau, v. 34, p. $56(1917) .-\mathrm{H}$. Stichel, Neue Beitr. syst. Ins.kunde, v. 3, p. 61 (1925).

forma gla ulias Fruhst. O. b. g., Fruh- Typ.: storfer: A. Seitz, Großschmett. Erde, v. 5, Sao Paulo p. 294 (1912). - Id., Ent. Rundschau, v. 34, p. 56 (1917). - o. g., H. Stichel, Neue Beitr. syst. Ins.kunde, v. 3, p. 64 (1925). 
forma be a ta Fruhst. O. batea beata, Fruh- Typ.: Santa storfer, Ent. Zeit. Stettin, v. 68, p. 140 Catharina (1907). - Id., Ent. Rundschau, v. 34, p. 56 (1917). - Id.: A. Seitz, Großschmett. Eide, v. 5, p. 294 (non subsericea, p. 1140, t. 61. a: ? = O. catharinae ㅇ) (1912). - Id., Ent. Rundschau, $\nabla .34$, p. 56 (1917). - II. Stichel, Neuo Beitr. syst. Ins.kunde, v. 3, p. 64 (1925) (forma dubia, an didymaon forma).

o. $d i d y m$ a on $d_{\text {. }}$ H. Stichel, Tierreich, Lief. 25, p. 57 (part. in cit.) (1909).

? O. b a tea, Zikán, Ent. Rundschau, v. 45, p. 10 (1928).

subsp. $s u b s e r i c e u s$ Fruhst. 0 . b. subseri- Rio Grande cea, Fruhstorfer, Ent. Zeit. Stottin, v. 68, do Sul p. 141 (1907). - H. Stichel, Berlin. Ent. Zeit., v. 52 (1907), p. 171 (1908). - 0 . b. subsericets, Id., Tior reich, Lief. 25, p. 55 (1909). - 0. b. subsericea, Fruhstorfer: A. Seitz, Großschmett. Erdo, v. 5, p. 294 (non t. 61a: ? = 0. catharinae Q) (1912). - Id., Ent. Rundschau, v. 34, p. 56 (1917). - H. Stichel, Neue Beitr. syst. Ins.kunde, v. 3, p. 64 (1925).

O. b a te $a$, Mabilde, Guia prat. Lep. Rio Gr. do Sul, p. 91 (not. biol.) (1896).

didymnon Feld. O. d., C. \& R. Felder, Reise Novara, Sao Paulov. 2. II, p. $453(1866)$. - H. Stichel, Rio Grande Berlin. Ent. Zeit., v. 52 (1907), p. 171 (1908). - Id., Tierreich, Lief. 25, p. 56 (1909), - 0. batea, Frulistorfer: A. Seitz, Großschmett. Erde, v. 5, p. 294 (part.) (1912).

subsp. di $d y \operatorname{ma}$ o $n$ Feld. 0. d., C. \& R. Fel- Sao Pauloder, ut antea (1866). - W. F. Kirby, Cat. Rio Grande Diurn. Irep., p. 126 (1871). - Chr. Auri- do Sul villius, Svenska Ac. Handl., n. ser., v. 19, Nr. 5, p. 75 (1882). - H. Stichel, Ins.Börse, v. 21, p. 197 (1904). - Id., Gen. Ins., Fasc. 20, p. $17(190 \mathrm{f})$. - Id., Soc. Ent., v. 22, p. 98 (1907). - Blepolenis d., Röber, Soc. Ent., v. 21, p. 19 (1906). Opsiphanes d. d., H. Stichel, Berlin. Ent. Zeit., v. 52 (1907), p. 171 (1908). - Id., Tierreich, Liof. 25, p. 57 (forma principalis) (cit. part.) (1909). - 0. batea d., Fruhstorfer: A. Seitz, GroBschmett. Erde, v. 5, p. 294 (1912). - O. d. d., id., Ent. Rundschau, v. 34, p. 56 (1917). - 0.d., H. Stichel, Neue Beitr. syst. Ins.kunde, v. 3, p. 63 (1925).

forma panorm $m$ (Röb.) Blepolenis didymaon var. p., Röber, Soc. Eut., v. 21, p. 20 (1906). - Opsiphanes d. var. p., H. Stichel do Sul, Paraguay (sec. Röber), Soc. Ent., v. 22, p. 98 (1907). - o. batea p., Fruhstorfer: A. Seitz, Großschmett. Erde, v. 5, p. 294 (1912).

o. didymaon p., id., Ent. Rundschau, จ. 34, p. 56 (1917). 
o. $d$ i $d y$ ma on $d$., H. Stichel, Tierreich, Lief. 25, p. 57 (part. in cit.) (1909).

forma $d u$ b i $a$ (Röb.) Blepolenis batea ab. $d$., Typ.: Röber, Soc. Ent., v. 68, p. 143 (1906). H. Stichel (sec. Röber), 1. c., v. 22, p. 98 (= didymann) (1907). - o. b. dubia, Fruhstorfer, Ent. Zeit. Stettin, v. 68, p. 143 (1907). - O. didymaon $d$. forma dubia, $\boldsymbol{H}$. Stichel, Berlin. Ent. Zeit., v. 52 (1907), p. 171 (1908). - Id., Tierreich, Lief. 25, p. 57 (1909). - O. batea dubia, Fruhstorfer: A. Seitz, Großschmett. Erde, v. 5, p. 294 (1912).

forma ga in a \& Fruhst. O. catherinae (!) g., Typ.: Fruhstorfer, Soc. Ent., v. 31, p. 59 (1916). Sao Paulo - O. c. gaivas (!), D. Sharp, Zool. Record 53 (1916), pars 12, p. 168 (1917). - $O$. catharinae gainas, Fruhstorfer Ent. Rundschau, v. 34, p. 56 (1917). - H. Stichel, Neue Beitr. syst. Ins.kunde, v. 3, p. 65 (? O. didymaon forma) (1925).

forma $e u c h l$ a en $a$ Fruhst. $O$. bates e., 0 . Typ.: didymaon e., Fruhstorfer, Ent. Rundschau, Sao Paulo v. 34, p. 55,56 (1917) (forma incertae sedis).

subsp. praegrandis Fruhst. O. batea p., Fruhstorfer, Ent. Zcit. Stettin, v. 68, p. 142 (1907). - O. didymaon p., II. Stichel, Berlin. Ent. Zeit., v. 52 (1907), p. 171 (1908). - Id., Tierreich, Lief. 25, p. 57 (1909). - O. batea p., Fruhstorfer: A. Seitz, Großschmett., v. 5, t. 61a; p. 295 (1911 \& 1912). - Id., Ent. Rundschau, v. 17, p. 56 (1917). - ? O. p., P. Köhler, Fauna Argent., pars 1 (Zeit. wiss. Ins.-Biol., v. 18, Beiheft), p. 21 (1923).

eatharinae Stich. $O$. c., H. Stichel, Berlin. Ent. Santa Zeit., v. 46 (1901), p. 505 (1902). - Id., Catharina, Neue Beitr. syst. Ins.kundc, v. 3, p. 65 Paraná (1925).

subsp. catharinae Stich. H. Stichel, ut Santa antea (1902). - Id., Gen. Ins., Fasc. 20, p. 18 (1904). - Id., Soc. Ent., v. 22, p. 98 (1907). - Id., Berlin. Ent. Zeit, v. 52 (1907), p. 171 (1908). - Blepolenis c., Röber, Soc. Ent., v. 21, p. 19 (1906). - Opsiphanes c., H. Stichel, Tierreich, Lief. 25, p. 57 (1909). - Fruhstorfer: A. Scitz, Großschmett. Ende, v. 5, p. 295 (1912). - Id., Ent. Rundschau, v. 34 , p. 65 (1917). - H Stichel, Neue Beitr. syst. Ins.kunde, v. 3, p. 65 ( $?=0$. batea forma beata part.) (1925).

Wilh el $m$ in a e (Röb.) Blepolenis c. var. $w$., Röber, Soc. Ent., v. 21, p. 19 (1906). - H. Stichel (sec. Röber), Soc. Ent., v. 22, p. 98 (= O. catharinae) (1907).

,s ubserice a", Fruhstorfer: A. Seitz, Großschmett. Erde, v. 5, t. 61 a (non p. 294) (Q) (1911). 
suhsp. Berchmansi Stich. O.c. b., H. Sti- Paraná chel, Neue Beitr. syst. Ins.kunde, v. 3, p. 65 (1925).

bassus Feld. 0. b., O. \& R. Felder, Reise Novara, Rio Grande v. 2. II, p. 453 (1866). - W. F. Kirby, do Sul Cat. Diurn. Lep., p. 125 (1871). - Chr. Aurivillius, Svenska Ak. Mandl., n. ser., v. 19, Nr. 5, p. 75 (1882). - O. Staudinger (\& Schatz), Exot. Schmett., v. 1, p. 212 (1887). - H. Stichel, Berlin. Ent. Zeit., v. 46 (1901), p. 504 (1902). - Id., Gen. Ins., Fasc. 25, p. 18 (1904). - o. b. b., Fruhstorfer, Ent. Zeit. Stettin, v. 68, p. 143 (1907). - 0. b., I. Stichel, Tierreich, Lief. 25 , p. 58, 59, f. 15 (forma principalis) $(1909)$. - $0 . \quad b . \quad b .$, Fruhstorfer: A. Seitz, Großschmett. Erdo, v. 5, p. 295 (1912). forma $l u t e i p e n n$ is Butl. O. l., A. G. Typ.: ?

Butler, Trans. Ent. Soc. London, p. 425 (1875). - W. F. Kirby, Cat. Diurn. Irep., Suppl., p. 716 (1877). - Chr. Aurivillius, Svenska Ak. Handl., n. ser., v. 19, Nr. 5, p. 75 (1882). - O. Staudinger (\& Schatz), Exot. Schinett., v. 1, p. 212 (1887). - 0 . bassus ab. $l$., H. Stichel, Berlin. Ent. Zeit., จ. 46 (1901), p. 501 (1902). - 0. b. forma l., id., Gen. Ins., Fasc. 20, p. 18 (1909). $0 . \quad b . l$., Truhstorfer, Ent. Zeit. Stettin, v. 68, p. 144 (1907). - O. b. ab. l., H. Stichel, Berlin. Ent. Zeit., v. 52 (1907), p. 172 (1908). - 0 . b. forma $l .$, id., Tierreich, Lief. 25, p. 60 (1909). - o. b. l., Fruhstorfer: A. Seitz, Großschmett. Erde, v. 5 , t. $61 a ;$ p. 295 (1911 \& 12).

forma o c ulata Stich. 0 . b. forma o., H. Typ.:

Stichel, Gen. Ins., Fasc. 20, p. 18 (1904). - Brasilien

Id., Tiemeich, Lief. 25, p. 60 (1909). -

o. b. o., Fruhstorfer: A. Seitz, Großschmett. Erde, v. 5, p. 295 (1912).

\section{b. Cohors Cassiiformes}

H. Stichel, Gen. Ins., Fasc. 20, p. 18 (1904). - Id., Soc. Ent., v. 22, p. 92 (1907). - Id., Tierreich, Lief. 25, p. 60 (1909).

Boisduralii Doubl. O. b., E. Doubleday (\& West- Mexikowood), Gen. Diurn. Lep., t. 57, f. 1 (1849). Honduras - O. b. (Meropis, Caligo b. Boisduval MS), J. O. Westrood, 1. c., v. 2, p. 345 (1851). Chenu \& H. Lucas, Enc. Hist. Nat., Pap. Diurn., p. 170 , f. 281 (1851-53). - Herrich-Schäffer, Corr.-Bl. Zool.-Min. Ver. Regensb., v. 19, p. 64 (1865). - Caligo b., Boisduval, Consid. Lép. Guatemala, p. 55 (1870). - Opsiphanes b., W. F. Kirby, Cat. Diurn. Lep., p. 126 (1871). - Id., Entomologist, v. 11, p. 26 (1878). - Id., Cat. Coll. Diurn. Lrep. Hewitson, p. 107 (1879). F. D. Godman \& O. Salvin, Biol. Centr.Amer., Lep.-Rhop., v. 1, p. 126; v. 2, p. 
664 (1881 \& 1901). - Chr. Aurivillius, Svenska Ak. Handl., n. ser., v. 19, Nr. 5, p. 76 (1882). - O. Staudinger (\& Schatz), Exot. Schmett., v. 1, p. 214 (1887). Witkugel, Soc. Ent., v. 5, p. 26 (1890). H. Stichel, Berlin. Ent. Zeit., v. 46 (1901), p. 506 (1902). - Id., Gen. Ins., Fasc. 20, p. 18 .(1904). - Id., Tierreich, Iief. 25, p. 60 (1909). - Fruhstorfer: A. Scitz, Grobschmett. Erde, v. 5, t. 62d; p. 295 (1911 $\& 12)$.

(L.) Papilio (Danaus) c., Linné, Syst. Nat., Süd-

ed. 10 , p. 471 (cit. part.) (1758). - Opsiphanes c., H. Stichel, Gen. Ins., Fasc. 20, p. 18 (1904). - Id., Tierreich, Lief. 25, p. 62 (1909). Fruhstorfer: A. Seitz, Großschmett. Erde, v. 5, p. 296 (1912).

Amerika, südl. bis

Bolivia,

Paraguay,

Argentinien

subsp. cassiac (L.) Papilio (Danaus) c., GuayanaLinné, ut antea (1758). - P. c., id., Mus. Colombia, Ludov. Ulr., p. 265 (1764). - O. Clerck, NordIcon. Ins., v. 2, t. 34, f. 2 (1764). $-P$. Brasilien, (Danaus) c., Linné, Syst. Nat., ed. 12, v. 1. Trinidad, II, p. 767 (1767). - Linné-Houttyn, Na- ? Peru tuurl. Hist., v. 1. XI, p. 270 (1767). P. c., P. S. I. Müller, Naturs. Linné, v. 5. I, p. 597 (1774). - (Gmelin \&) Christman, Onomatol. Med., v. 6, p. 48 (1775). J. C. Fabricius, Syst. Ent., p. 483 (cit. part.) (1775). - „Cassine", $P$. c., P. Oramer, Pap. Exot., v. 2, p. 13, t. 105, f. A, B (Q); p. 148 (1777). - P. (Danaus) c., Groere, Ent. Beytr., v. 3. I, p. 193 (cit. part.) (1779). - P. c., J. C. Fabricius, Spec. Ins., v. 2, p. 58 (cit. part.) (1781). - O. Stoll, Essai Syst. Lep. (P. Cramer, Pap. Exot., v. 4, App.), p. 8, Nr. 22 (part.) (1782). P. (D)anaus Festivus) c., [D. II. Schneider], Nomencl. Entom., p. 35 (1785). - P. c., Fabricius, Mant. Ins., v. 2, p. 281 (cit. part.) (1787). - P. (Danaus) c., GmelinLinné, Syst. Nat., ed. 13, v. 1. V, p. 2280 (cit. part.) (1790). - P. c., C. Stoll, Suppl. Oramer, Pap. Exot., p. 16 (cit. part.), t. 3, f. $3 A, B$ (larva, chrysal.) (conf. WV. Müller, Zool. Jahrb., v. 1, p. 594, 18S6) (1791). - P. c., Jung, $\Lambda$ lphab. Verz. Schmett., v. 1, p. 106 (cit. part.) (1791). - (Jablonsky \&) Herbst, Naturs. Ins., Schmett., v. 6, p. 54 (cit. part.), t. 129, f. 1, 2 (q) (non f. $3:=0$. cassina merianae $)(1793)$. - Fabricius, Ent. syst., v. 3. I, p. 150 (cit. part.); Index alphab., p. 120 (1793 \& 96). - Fr. Weber, Nomencl. IBntom., p. 101 (1795). - P. cassid (!), Tableau Enc. Méth., v. 18, t. $25, \mathrm{f} .3,3$ bis; f. $4 \mathrm{~A}, \mathrm{~B}$ (larva, chrysal.) (1797). - $P$. cassine, Iatreille, Hist. Crust. Ins., จ. 3, p. 392 (1802). - P. (Brassolis) c., Iatreille, Gen. Crust. Ins., v. 4, p. 195 
(1806-09). - "Potamis superba c.", Jac. Hübner, Samml. Exot. Schmett., v. 1, t. [74] $\left(\sigma^{7}\right)$, t. [75] () $(1806-19)$. $-P . c$. , Glavenhorst, Vergl. Ubersicht Zool. Syst., p. 313 (part.) (1807). - Latreille: Cuvier, Règne An., v. 3, p. 545 (1817). - Brassolis c., Jac. Hübner, Verz. Schmett., p. 50 (1818). - Id., Index Exot. Schmett., v. 2, p. [2] (1821). - Morpho c., Pavonia c., (Latreille \&) J. B. Godart, Enc. Méth., v. 9, p. 454, 807 (1824). - Brassolis c., J. G. Children (sec. Trabr.), Philos. Mag., v. 7, p. 120 (1830). - Pavonia c., Verioren, Cat. Ins. Lep. Cramer, ₹. 1, p. 50 (sine cit.); v. 2, p. 202 (part.) (1837). - E. Blanchard, Hist. An. Artic., v. 3, p. 454 (cit. part.) (in syn. falso Pap. hycerie pro glycerie) (1840). - E. Doubleday, List Lep. Brit. Mus., v. 1, p. 118 (1844). - E. Blanchard, Hist. Ins., v. 2, p. 340 (1845). - L[ucas], Orbigny, Dict. Hist. Nat., v. 9, p. 515 (cit. part.) (1847). - Opsiphanes c. (1. Doubleday \&) J. O. Westwood, Gen. Diurn. Lep., v. 2, p. 346 (cit. part.) (1851). - Ménétriés, Enum. Corp. Anim. Ac. Petropol., pars 1, p. 38 (1855). - ? H. Lucas: Sagra, Hist. fis. Cuba, v. 7, p. 582 (1857). - C. \& R. Felder, Wien. Ent. Monatsehr., v. 5, p. 111 (1861). - Papilio c. (Opsiphanes), W. F. Kirby, Trans. Ent. Soc. London, p. 143 (recogn. Linnéi typ.) (1870). - Caligo c., Capronnier, Ann. Soc. Ent. Belg., v. 17, p. 28 (1874). - ? O. c., Herb. Druce, Proc. Zool. Soc. London, p. 217 (1876). - ? W. F. Kirby, Cat. Coll. Diurn. Lep. Hewitson, p. 107 (1879). - Chr. Aurivillius, Svenska Ak. Handl., n. ser., v. 19, Nr. 5, p. 72, 76 (cit. nonnull. except.) (1882). - I. Glaser, Cat. Etymol. Col. Lep., p. 301 (1887). ? Röber: O. Staudinger \& Schatz, Exot. Schmett., จ. 2, t. 32; p. 190, 191 (Morphol.) (1888 \& 89). - Weymer (\& Maassen), Lep. Reise Stübel, p. 20, 90 (1890). - Poujade, Bull. Soc. Ent. France, v. 64, p. CXLIII (1895). - - H. Stichel, Berlin. Ent. Zeit., v. 46 (1901), p. 506 (1902). - IV. J. Kaye, Trane. Ent. Soc. London, p. 165 (1904). Guppy, 1. c., p. 226, t. 17, f. 2 (larva) (1904). - O. c. c., H. Stichel, Gen. Ins., Fasc. 20, p. 18 (1904). - O. c., Fruhstorfer, Ent. Zeit. Stettin, v. 68, p. 136 (1907). o. c. c., H. Stichel, Berlin. Ent. Zeit., v. 52 (1907), p. 168 (1908). - Id., Tierreich, Lief. 25, p. 64, f. 16 (cit. corrigend.) (1909). - Fruhstorfer: A. Seitz, Großschmett. Erde, v. 5, p. 296 (1912).

glycerie (Fabr.) Papilio g., J. C. Fabricius, Mant. Ins., v. 2, p. 36 (eit. part.) (1787). - Pap. (Nymphalis) g., Gmelin- 
Linné, syst. Nat., ed. 13, v. 1. V, p. 2307 (1790). - P. g., Jung, Alphab. Verz. Schmett., v. 1, p. 45 (1791). - J. O. Fabricius; Ent. syst., v. 3. I, p. 94; Index alphab., p. 123 (1793 \& 96). - Turton, Gen. Syst. Nat. Linné, v. 3. II, p. 92 (1806). - W. F. Kirby: Hübner \& Geyer, Samml. Exot. Schmett., Neue Ausg., v. 3, p. 51 (1901).

Orameri Feld. O. c., O. \& R. Felder, Wien. Ent. Monatschr., v. 6, p. 123 (1862). Herrich-Schäffer, Corr.-Bl. Zool.-Min. Ver. Regensb., v. 19, p. 64 (1865). - W. T. Kirby, Cat. Diurn. Lep., p. 126 (part. in cit.: Pap. glycerie F.) (1871). - ? Möschler, Verh. Zool.-Bot. Ges. Wien, v. 26, Abh., p. 321 (1877). - W. F. Kirby, Cat. Coll. Diurn. Lep. Hewitson, p. 107 (part.) (1879). - O. Staudinger (\& Schatz), Exot. Schmett., v. 1, p. 213 (1887). - E. Reuter, Acta Soc. Sci. Fonn., v. 22, p. 112 (1896). - Fassl, Soc. Ent., v. 27, p. 54 (orum) (1912).

$P$ a pilio $x$ anthus (non Linné), Thunberg, Mus. Nat. Ac. Upsal., pars 23, p. 9 (sec. Aurivillius, 1882) (1804).

Pavonia cas \&iae (non Pap. c. Linné), L[ucas]: Orbigny, Dict. Hist. Nat., v. 9, p. 515 (part. in cit.: hycerie (!) F.) (1847).

opsiphanes quiteria (non Pap. qu. Stoll), W. F. Kirby, Cat. Diurn. Lep., p. 126 (part.) (1871). - Weymer (\& Maassen), Iep. Reise Stübel, p. 100 (part. in cit.) (1890).

forma cas siculus Stich. 0 . cassiae cassi- Typ.: culus, H. Stichel, Gen. Ins., Fasc. 20, p. 19
(1904). - Frulistorfer, Ent. Zeit. Stettin, v. 68, p. 136 (1907). - O. cassiae c. forma cassiculus, H. Stichel, Berlin. Ent. Zeit., v. 52 (1907), p. 170 (1908). - Id., Tierreich, Lief. 25 , p. 66 (1909). - 0 . cassiae cassiculus, Frnhstorfer: A. Seitz, Großschmett. Erde, v. 5, p. 296 (1912).

$p s e u d$ ocassiae Frulst. 0 . tamarindi $p$. Fruhstarfer, Ent. Zeit. Stettin, v. 68, p. 140 (1907). - O. cassiue $p_{.}$id.: A. Seitz, Großschmett. Erde, v. 5, p. 296 (1912).

subsp. $p$ udicus Fruhst. O. c. forma p., Bahia Fruhstorfer, Ent. Zeit. Stettin, v. 68, p. 136 (1907). - O. c. c. forma p., H. Stichel, Berlin. Ent. Zeit., v. 52 (1907), p. 168 (1908). - Id., Tierreich, Lief. 25, p. 65 (1909). - O. c. p., Fruhstorfer: A. Soitz, Großschmett. Erde, v. 5, p. 296 (1912) (subsp. dubia).

subsp. decentius Fruhst. O. c. forma d., Minas Geraes Fruhstorfer, Ent. Zeit. Stettin, v. 68, p. 137 (1907). - O. c. c. forma d., H. Stichel, Berlin. Ent. Zeit., v. 52 (1907), p. 168 (1908). - Id., Tierreich, Lief. 25, p. 65 
(1909). - O. c. d., Fruhstorfer: A. Seitz, Großschmett. Eirde, v. 5, p. 296 (1912). H. Stichel, Neue Beitr. syst. Ins.kunde, v. 3, p. 68 (1925) (vix conserv.)

subsp. $l$ u c u $l l$ u s Fruhst. O. c. l., Truhstor- Espirito Santo

fer, Ent. Zoit. Stettin, v. 68, p. 136 (1907). -Santa

- Id.: A. Seitz, Großschmett. Erde, v. 5, t. Catharina,

61a; p. 296 (1911 \& 12). - W. Rothschild, Paraná,

Nov. Zool., v. 23, p. 311, t. 5, f. 5, 7 Paraguay,

(chrysal., larva) (1916). - o. l., P. Köhler, Argentinien

Fauna Argent., pars 1 (Zeit. wiss. Ins.-Biol., v. 18, Beihoft), p. 21 (1923). - O. cassiae-

l., Zikán, Ent. Rundschau, v. 45, p. 10 (1928).

- Eltringham, Trans. Ent. Soc. London,

v. 77, p. 1-4, fig. (Physiol.) (1929).

o. cassiae (non Pap. c. Linn仑), C. \& R. Felder, Wien. Ent. Monatschr., v. 6, p. 123 (1862). - Id., Verh. Zool.-Bot. Ges. Wien, v. 12, Abh., p. 476 (1862). - Prittwitz, Ent. Zeit. Stettin, v. 26, p. 309 (1865). - $\mathrm{Pa}$ vonia c., Burmeister, Rov. Mag. Zool., ser. 3, v. 1 , p. 31 , t. 1 , f. 9 (larvao caput), t. 6, f. 1, 2 (larvae); p. 42 (1873). - Caligo c., Capronnier, Ann. Soc. Ent. Belg., v. 17, p. 28 (Phaonol.) (1874). - Opsiphanes c., K. (sec. F. Müller), Kosmos, Leipzig, v. 1, p. 260 (Physiol.: odor) (1877). - Bönninghausen, Verh. Ver. Naturw. Unterh. Hamburg, v. 9 (1894-95), p. 39 (1896). - C. Berg, An. Mus. Nac. Buenos Aires, v. 6, p. 371 (1899). - O. c. c., H. Stichel, Berlin. Ent. Zeit., v. 52 (1907), p. 168 (1908). Id., Tierreich, Lief. 25, p. 64 (part. in cit.) (1909). - ? O. c. c., Strand, Soc. Ent., v. 33 , p. 19 (1918).

o. glycerie (non Pap. g. Fabr.), Dukinfield Jones (\& F. Moore), Proc. Lit. Philos. Nat. Hist. Soc. Liverpool, v. 36, p. 338 (sine cit.), t. 3, f. 4-6 (Metamorph.) (1882).

subsp. strophios Fruhst. O. c. s., Fruhstor- Bolivia fer, Ent. Zeit. Stettin, v. 68, p. 137 (1907). - H. Stichel, Berlin. Ent. Zeit., v. 52 (1907), p. 168 (1908). - Id., Tierreich, Lief. 25, p. 67 (1909). - Schrottky, Ent. Vereinsbl. (Ent. Rundscliau, v. 26, Beil.), p. 43 (? part., couf. O. tamarindi terenzius) (1909). - Fruhstorfer: A. Seitz, Großschmett. Erde, v. 5, p. 296 (1912).

subsp. $r$ r $b i g a t u s$ Stich. O. c. $r$., H. Sti- Ecuador

chel, Gen. Ins., Fasc. 20, p. 19 (1904). - (Coca)

Id., Tierreich, Lief. 25, p. 67 (1909). Fruhstorfor: A. Seitz, Großschmett. Ende, v. 5, p. 296 (1912). - Strand, Soc. Ent., v. 33, p. 19 (1918).

? O. c a s s ia e, Dognin, Lép. Loja, p. 34, 72 (1891 \& 94). - Campos, Rev. Col. Nac. Vicente Rocafuerte, Nr. 4, p. 31 (1921). 
subsp. eastaneus Stich. O. cassiae castaneus, H. Stichel, Gen. Ins., Fasc. 20, p. 19 (1904). - Id., Tierreich, Lief. 25, p. 66 (1909). - ? Fassl, Fauna Exot., v. 1, p. 26 (1911). - Frubstorfer: A. Seitz, Großschmett. Erde, v. 5, p. 296 (1912).

? O.glycerie (non Pap. g. Fabr.), A. G. Butler, Cat. Diurn. Lep. Fabl., p. 41 (part.) (1869).

O. Crameri (non Feld.), Tristan, Ins. Costa Rica, p. 18 (1897).

? O. cassiae (non Pap. c. Linné), H. G. Dyar, Proc. U. S. Nat. Mus., v. 47, p. 144 (1914).

zelotes Hew. O. z., Hewitson, Exot. Butterfl., v. 4 Colombia, (5), t. [7], Opsiphanes 1 (1873). - H. MittelStichel, Tierreich, Lief. 25, p. 68 (1909). Amerika - Fruhstorfer: A. Seitz, Großschmett. Erde, v. 5, p. 296 (1912).

subsp. zelotes Hew. O. z., Hewitson, ut Colombia antea, f. 3, 4 (1873). - W. F. Kirby, Cat. Diurn. Lep., Suppl., p. 716 (1877). Id., Cat. Coll. Diurn. Lep. Hewitson, p. 31 (1879). - Chr. Aurivillius, Srenska Ak. Handl., n. ser., v. 19, Nr. 5, p. 76 (1882). - O. Staudinger (\& Schatz), Exot. Schmett., v. 1, p. 213 (1887). - H. Stichel, Berlin. Ent. Zeit., v. 46 (1901), p. 511 (1902). Id., Gen. Ins., Fasc. 20, p. 19 (1904). O. z. z., id., Tierreich, Lief. 25, p. 68 (1909). - Fruhstorfer: A. Seitz, Großschmett. Erde, v. 5, p. 296 (1912).

0 . zelys (non 0 . zelotes zelus Stich.), Fassl, Ent. Rundschau, v. 35, p. 31 (1918).

subsp. z e $l u s$ Stich. 0 . zelotes zelus, H. Sti- Panama chel, Berlin. Ent. Zoit., v. 52 (1907), p. 174 (Chiriqui) (1908). - Id., Tierreich, Lief. 25, p. 68 (1909). - Fruhstorfer: A. Seitz, Großschmett. Erde, v. 5, p. 297 (1912).

- tamarindi Feld. O. t., C. \& R. Felder, Wien. Ent. Venezuela, Monatschr,, v. 5, p. 111 (1861). - H. Sti- Mittelchel, Berlin. Ent. Zeit., v. 46 (1901), p. 509 Amerika(part.) (1902). - Id., Gen. Ins., Fasc. 20, Bolivia, p. 19 (part.). - Id., Tierreich, Lief. 25, ? Santa p. 68 (part.). - Fruhstorfer: A. Seitz, Catharina Großschmett. Erde, v. 5, p. 297 (part.) (1912).

subsp. tamarindi Feld. O. t. (Boisduval Venezuela, MS), C. \& R. Felder, Wien. Ent. Monatschr., v. 5, p. 111 (1861). - Herrich-Schäffer, Corr.-Bl. Zool.-Min. Ver. Regensb., ఛ. 19, p. 64 (1865). - Caligo t., Boisduval, Consid. Lép. Guatemala, p. 54 (1870).-Ops. t., W. F. Kirby, Cat. Diurn. Lep., p. 126, 646 (1871). - Id., Cat. Coll. Diurn. Lep. Hewitson, p. 107 (part.) (1879). - O. tamarinde (I), F. D. Godman \& O. Salvin, Trans. Ent. Soc. London, p. 122 (1880). - O. tamarindi, iid., Biol. Centr.-Amer., Lep.-Rhop., v. 1, p. 
128 (cit. part.), t. 13, f. 5, 6; v. 2, p. 664 (1881 \& 1901). - Chr. Aurivillius, Svenska Ak. Handl., n. ser., v. 19, Nr. 5 , p. 76 (1882). - O. Staudinger" (\& Schatz), Exot. Schmett., v. 1, p. 213 (part.) (non to 72: $=0 . t$. terenzius) (1887). - Hahncl, Deutsch. Ent. Zeit. Lep. (Iris), v. 3, p. 166 (1890). - o. t. t., H. Stichel, Gen. Ins., Fasc. 20, p. 19 (1904). - Id., Soc. Ent., v. 22, p. 99 (1907). - Id., Tierrcich, Lief. 25, p. 69 (forma principalis) (cit. nonull. except.) (1909). - O. t., Fassl, Faun. Exot., v. 1 , p. 26 (distr. vertical.) (1911). Id., Ent. Rundschau, v. 33, p. 26 (1912). - Gibbs, Proc. Ent. Soc. London, p. XLVIII (1912). - 0. t. t., Fruhstorfer: A. Seitz, Großschmett., v. 5, t. 61 b $(\sigma$, 우 $)$ p. 297 (sine synon.) (1911 \& 12). - o. t., Fassl, Ent. Rundschau, v. 33, p. 26 (1916). . O. $t$. $t$. forma typica, H. Stichol, Neue Beitr. Ins.kunde, v. 3, p. 66, 67 (1925).

sikyon Fruhst. 0. t. s., Fruhstorfer: $A$. Seitz, Großschmett. Erde, v. 5, p. 297 (1912). - Jurriaanse, Tijdschr. Ent., จ. 66, p. 146 t. 2, (Physiol.) (1923).

forma $x$ i $p \not$ o 8 Fruhst. $0 . t$. $x$, Fruhstorfer, Typ.:

Ent. Zeit. Stettin, v. 68, p. 140 (1907). - Venezuela $O$. $t$. $t$. forma $x$., H. Sticliel, Berlin. Ent. Zeit., v. 52 (1907), p. 169 (1908). - Id., Tierreich, Lief. 25, p. 70 (1909). - 0. x., Fruhstorfer: A. Seitz, Großschmett. Erde, v. 5, t. 61b (1911). - o. t. xiphios (!), o. t. t. forma xiphos, H. Stichel, Neue Beitr. syst. Ins.-kunde, v. 3, p. 66, 67 (1925).

o. tamarindi t., Fruhstorfer: A. Seitz, Großschmett. Erde, v. 5, p. 297 (in synon.) (1912).

forma $m$ es o merista Stich. 0 . $t$. $t$. forma Typ.: $m ., H$. Stichel, Neue Beitr. syst. Ins.kunde, Venezuela v. 3, p. 66,67 (1925).

subsp. Ki le ist hemes Fruhst. O. t. k., Fruh- Weststorfer, Ent. Rundschau, v. 29, p. 15 (1912). Colombia

- Id.: A. Seitz, GroBschmett. Erde, v. 5, p. 297 (1912). - Fassl, Ent. Rundschau, v. 33, p. 26 (1916). - H. Stichel, Neue Beitr. syst. Ins.kunde, v. 3, p. 67 (1925).

O. tamarindi t., H. Stichel: Tierreich, Lief. 25, p. 69 (part.) (1909).

subsp. cherocles Fruhst. O. t. c., Fruh- Oststorfer, Ent. Rundschau, $\nabla .29$, p. 15 (1912). Colombia - Id.: A. Seitz, Großschmett. Erde, v. 5, (Ober. Rio p. 297 (1912). - Fassl, Ent. Rundschan, v. Negro) 33, p. 26 (1916) - O. c., id., l. c., v. 35, p. 31 (1918). - O. t. c., H. Stichel, Neue Beitr. syst. Ins.-kunde, v. 3, p. 67 (1925).

subsp. corrosus Stich. 0 . $t$. c., H. Stichel, Ecuador Ins.-Börse, v. 21, p. 21 (1904). - Id., Gen. Ins., Fasc. 20, p. 19 (forma principalis) 
(1904). - Id., Soc. Ent., v. 22, p. 99 (1907). - Id., Tierreich, Lief. 25, p. 70 (1909). Fruhstorfer: A. Seitz, Großschmett. Ende, v. 5, p. 297 (1912). - Strand, Lep. Niepeltiana, v. 2 , p. 12 (1916). - O. $t$. c., forma typica, H. Stichel, Neue Beitr. syst. Ins.-kunde, v. 3, p. 67 (1925). - O. c., Campos, Rer. Col. Nacion. Vicente Rocafuerte, Nr. 4, p. 31 (1921).

o. tamarind $i$, Weymer (\& Maassen), Lep. Reise Stübel, p. 61, 65 (1890). - F. D. Godman \& O. Salvin: Whymper, Trav. Great Andes, App., p. 99 (1892). - H. Stichel, Berlin. Ent. Zeit., v. 46 (1901), p. 510 (1902). - Id., 1. c., v. 47, Sitz.-Ber., p, (21) (1902).

ah. $p s e u d o s p a d i x$ Strand. $P$. $t$. corrosus "Form." $p$., Strand, Lep. Niepeltiana, v. 2, p. 13 (1916). - o. t. c. ah. ps., id., Bull. Soc. Zool. France, v. 51, p. 398 (1926).

forma $s p a d i x$ Stichel. $O$. tamarindi $t$. ab. Typ.: s., II. Stichel, Berlin. Ent. Zeit., จ. 46, Balzapamba (1901), p. 510 (1902). - o. t. corrosus forma s., id., Gen. Ins., Fasc. 20, p. 19 (1909). - Id., Tierreich, Lief. 25, p. 71 (1909). - Fruhstorfer: A. Seitz, Großschmett., v. 5, p. 297 (1912). - H. Stichel, Neue Beitr. syst. Ins.kunde, v. 3, p. 67 (1925).

suhsp. ierenzius Fruhst. 0 . tamarindi te- Santa renzizs, Fruhstorfer, Großschmett. Erde, v. Catharina 5, p. 297 (1912). - I. Stichel, Neue Beitr. syst. Ins.kunde, v. 3, p. 67 (subsp. duhia) (1925).

? O. tamarindi, G. W. Müller, Zool. Jahrb. Syst., v. 1, p. 593 (Biol.) (1886). - O. Staudinger (\& Schatz), Großschmett. Erde, v. 1, t. 72 ; p. 213 (part.) (1886 \& 87). - o. t. t., II. Stichel, Tierreich, Lief. 25, p. 69, 70 (Biol. sec. W. Müller) (1909).

? O. cassiae strophios (non Fruhst.), Sehrottky, Entom. Ver.-Blatt, Entom. Rundschau, v. 26, Beil.), p. 43 (1909).

- bogotanus Dist. O. b., Distant, Ent. Monthly Mag., v. 11, p. 203 (1875). - Fruhstorfer: A. Seitz, Großschmett. Erde, v. 5, p. 298 (1912).

o. t a marindi, H. Stichel, Gen. Ins., Fase. 20, p. 19 (part.) (1904). - Id., Tierreich, Lief. 25, p. 68 (part.) (1909).

suhsp. $b$ ogotan $u s$ Dist. 0 . b., Distant, ut Colombia autea (1875). - W. F. Kirhy, Cat. Diurn. Lep., Suppl., p. 716 (1877). - F. D. Godman \& O. Salvin, Trans. Ent. Soc. London, p. 122 (1880). - C. O. Waterhouso, Identif. Ins., v. 1, t. 55 (1881). - - ? O. Staudinger (\& Schatz), Exot. Schmett., v. 1, p. 213 (1887). - Weymer (\& Maassen), Lep. Reise Stühel, p. $33(1890)$. - O. tamarindi b., H. Stichel, Berlin. Ent. Zeit., v. 46 (1901), p. 510 
(1902). - Id., Gen. Ins., Fasc. 20, p. 19 (1904). - Id., Soc. Ent., v. 22, p. 99 (1907). - O. b., Fassl, Soc. Ent., v. 24, p. 115 (Metam.); v. 27 , p. 54 (1909 \& 12). - 0. tamarindi b., H. Stichel, Tierreich, Lief. 25, p. 71 (1909). - O. b. b., Fruhstorfer: A. Seitz, Großschmett. Erde, v. 5, p. 298 (1912). - W. Rothschild, Nov. Zool., v. 23, p. 311, t. 6, f. 5,6 (larva, chrysal.) (1916). -0 . b., Fassl, Ent. Rundschau, v. 33, p. 26 (1916). - H. Stichel, Neue Beitr. syst. Ins.kunde, v. 3, p. 68 (1925).

A u r ivilli i Röb. O. a., Röber, Soc. Ent., v. 21, p. 20 (1906).

subsp. phrataphernes Fruhst. O. b. p, OstFruhstorfer, Ent. Rundschau, v. 29, p. 15 (1912). - Id.: A. Seitz, Großschmett. Erde, v. 5, p. 299 (1912). - Fassl, Ent. Rundschau, v. 33 , p. 26 (1916). - O. prataphernes (!), id., l. c., v. 35 , p. 31 (1918).

subsp. in columis Stich. O. tamarindi i., H. Peru, Stichel, Ins.-Börse, v. 21, p. 21 (1904). - Bolivia Id., Gen. Ins., Fasc. 20, p. 19 (1904). - Id., Soc. Ent., v. 22, p. 99 (1907). - Id., Tierreich, Liof. 25 , p. 72 , f. 17 (q) (1909). Fruhstorfer: A. Seitz, Großschmett. Erde, v. 5, p. 297 (1912). - O. bogotanus i., H. Stichel, Neue Beitr. syst. Ins.kunde, v. 3, p. 68 (1925).

per u a $n$ u \& Rothsch. O. b. p., W. Rothschild, Nov. Zool., v. 23, p. 311 (1916).

forma lat if as cia Rothsch. O. txmarindi l., Typ.:

W. Rothschild, Nov. Zool., v. 23, p. 311 Süd-Ost-

(1916). - H. Stichel, Neue Beitr. syst. Ins.- Peru

kunde, v. 3, p. 68 (1925).

(Oroya)

quiteria (Stoll). Papilio q., C. Stoll: P. Cramer, Pap. GuayanaExot., v. 4, p. 251 (1782). - Opsiphancs q., Paraguay, II. Stichel, Berlin. Tint. Zeit., v. 46, (1901), p. 511 (1902). - Id., Gen. Ins., Fašc. 20, p. 20 (part.) (1904). Id., Tierreich, Lief. 25, p. 73 (part.) (1909). - Fruhstorfer: A. Seitz, Großschmett. Erde, v. 5, p. 298 (1912).

subsp. quiteria (Stoll) - Seba, Thesaurus, v. Guayana.

4 , p. 21 , t. 15 , f. 10,11 (sec. Aurivillius, Ost-

$1882,=0$. invirae, sed errans puto) (1765). Amazonas,

- "Quiteria", Papilio q., C. Stoll, ut antea, ? Colombia

p. 49 , t. 313 , f. A-D; $P_{\text {. }} q .$, id., Essai Syst.

Iittel-

Amerika-

Bolivia

Lep., 1. o., App., p. 8, Nr. 21 (1780 \& 82).

- P. (Nymphalis Gemmatus) q., [D. H.

Schneidor 7 , Nomencl. Entom., p. 37 (1785).

- P. (Danaus) xinthrs, $\beta$. ceutcria (laps. typ.), Gmelin-Linné, Syst. Nat., ed. 13, v. 1. V, p. $2282(1790)$. - P. quiteria, Jung, Alphab. Verz. Schmett., v. 2, p. 159 (1792). - P. quiterius (!), (Jablonsky \&) Herbst, Naturs. Ins., Schmett., v. 6, p. 50, t. 127 , f. 3,4 (9); t. 128 , f. 1, 2 (O) (1793). - P. 
quiterie (!), Tableau Enc. Méth., v. 18, t. 25, f. 2, 2bis (1797). - Brassolis quiteria, Jac. Hübner, Verz. Schmett., p. 50 (sine cit.) (1818). - Pavonia q., Verloren, Cat. Ins. Lep. Cramer, v. 1, p. 117 (1837). - Opsiphanes q., Herrich-Schäffer, Corr.-Bl. Zool.Min. Ver. Regensb., v, 19, p. 64 (1865). W. F. Kirby, Cat. Diurn. Iep., p. 126 (cit. part.) (1871). - Möschler, Verh. Zool.-Bot. Ges. Wien, v. 26, Abh., p. 321 (1877). - A. G. Butler, Trans. Ent. Soc. London, p. 114 (1877). - ? W. F. Kirby, Cat. Coll. Diurn. Lep. Hewitson, p. 107 (1879). - Chr. Aurivillius, Svenska Ak. Handl., n. ser., v. 19, Nr. 5, p. 76 (1882). - O. Staudinger (\& Schatz), Exot. Schmett., v. 1, p. 213 (1887). - ? Weymer (\& Maassen), Lep. Reise Stübel, p. 100 (cit. part.) (1890). - E. Reuter, Acta Soc. Sci. Fenn., v. 22, p. 112 (1896). - O. q. q., H. Stichel, Berlin. Ent. Zeit., v. 46 (1901), p. 511 (part.) (1902). Id., Gen. Ins., Fasc. 20, p. 20 (1904). Fruhstorfer, Int. Ent. Zeit., v. 1, p. 30 (1907). - H. Stichel, Berlin. Ent. Zeit., v. 52, p. 167 (part.) (1908). - Id., Tierreich, Lief. 25, p. 75 (cit. part.) (1909). Fruhstorfer: A. Seit\%, Großschmett. Erde, v. 5, t. $61 \mathrm{~b} ;$ p. 298 (1911 \& 12).

$P a p i l i a x a n t h u s$ (non Linné), J. C. Fabricius, Mant. Ins., v. 2, p. 296 (? part.) (1787).

Morpho cassiae, Pavoniac., (Latreille \&) J. B. Godart, Enc. M[éth., v. 9, p. 454 (part. in cit.) (1824). - P. c., Verloren, Cat. Ins. Lep. Cramer, v. 2, p. 202 (1837). - E. Blanchard, Hist. An. Artic., v. 3, p. 454 (part. in cit.) (1840). - Opsiphanes c., (E. Doubleday \&) J. O. Westwood, Gen. Diurn. Lep., จ. 2, p. 346 (part. in cit.) (1851).

subsp. o $b$ id on $n s$ Fruhst. $0 . q$. o., Fruh- Amazonas storfer, Ent. Zeit. Stettin, v. 68, p. 134 (1907). - Id., Int. Ent. Zeit., v. 1, p. 30 (1907). - Id.: A. Seitz, Großschmett. Erde, v. 5, t. $62 \mathrm{~d}$ (abidonius), p. 298, 1134, 1140 (1911, 12, 24).

? O. $q u$ iteria, Hahnel, Deutsch. Ent. Zeit. Lep. (Iris), v. 3, p. 249 (1890).

o. $q u$ iteria q., H. Stichel, Tierreich, Lief. 25, p. 75 (part. in cit.) (1909).

subsp. $p h y$ las Fruhst. O. q. p., Fruhstorfer, OstEnt. Rundschau, v. 29, p. 15 (1912). - Id.: Colombia A. Seitz, Großschmett. Erde, v. 5, p. 299 (1912). - Fassl, Fnt. Rundschau, v. 33, p. 26 (1916). - o. p., id., 1. c., v. 35, p. 31 (1918) (Subsp. dubia).

subsp. $a$ ug eias Fruhst. 0 . q. a., Fruhstorfer, Ent. Rundschau, v, 29, p. 15 (1912). Id.: A. Seitz, Großschmett. Erde, v. 5, p. 299 (1912). - O. a., Fassl, Ent. Rundschau, 
v. 31 , p. 41 (1914). - 0 . angias (!), id., 1. c., v. 33, p. 26 (1916).

subsp. $q u$ irinus Godm. \& Salv. O. quiri- Mittelnus, F. D. Godman \& O. Salvin, Biol. Amerika Centr.-Amer., Lep.-Rhop., v. 1, p. 128; v. 2, p. 665 (1881 \& 1901). - Chr. Aurivillius, Svenska Ak. Handl., n. ser., v. 19, Nr. 5, p. 76 (1882). - O. quiteria var. quirinus, $\mathrm{O}$. Staudinger (\& Schatz), Exot. Schmett., v. 1, p. 213 (1887). - o. quit. quir., H. Stichel, Berlin. Ent. Zeit., v. 46 (1901), p. 512 (1902). - Id., Gen. Ins., Fasc. 20, p. 20 (1904). - Fruhstorfer, Int. Ent. Zeit., v. 1, p. 30 (1907). - H. Stichel, Soc. Ent., v. 22, p. 99 (1907). - Id., Berlin. Int. Zeit., v. 52 (1907), p. 165 (1908). - Id., Tierreich, Lief. 25, p. 77 (1909). - Fruhstorfer: A. Seitz, Großschmett. Ende, v. 5, p. 298 (1912).

Caligo quiteria, Boisduval, Consid. Lép. Guatemala, p. 54 (1870).

? Opsiphanas glycerie (non Pap. g. Fabr.), Distant, Proc. Ent. Soc. London, p. XIII (1876).

subsp. $q u$ a es tor Stich. O. quiteria qunestor, Ost-

H. Stichel, Berlin. Ent. Zeit., v. 46 (1901), Ecuador, p. 512, f. 1 (1902). - Id., Gen. Ins., Fase. Peru 20, p. 20 (1904). - Id., Berlin. Ent. Zeit., v. $52,(1907)$, p. 166 (1808). - Id., Tierreich, Lief. 25, p. 77 (part.) (1909). Fruhstorfer: A. Seitz, GroBschmett. Erde, v. 5, p. 299 (1912).

? O. quiteria, Dognin, Ĺép. Loja, p. 72 (1894).

o. $q u$ ir in us (non Godm. \& Salv.), Campos, Rev. Col. Nacion. Vicente Rocafuerte, Nr. 4, p. 31 (1921).

forma $m$ y las a Fruhst. $O . q$. m., Fruhstor- Typ.: fer, Ent. Zeit. Stettin, v. 68, p. 133 (1907). West- Id., Int. Ent. Zeit., v. 1, p. 30 (1907). - Amazonas Id.: A. Seitz, Großschmett. Erde, v. 5, p. (Rio Uaupes) 298 (1912).

0 . $q u$ iteria qua stor, H. Stichel, Tierreich, Lief. 25, p. 77 (part. in cit.) (1909).

subsp. quirinalis Staud. O. quiteria var. Süd-Peru quirinalis, O. Staudinger (\& Schatz), Exot. iSchmett., v. 1, p. 213 (part.) (1887). O. quit. quirin. ab. quirinal., H. Stichel, Berlin. Ent. Zeit., v. 46 (1901), p. 512 (1902). - o. quit. quirin., id., Gen. Ins., Fasc. 20, p. 20 (1904). - Id., Soc. Ent., v. 22, p. 99 (1907). - Id., Int. Ent. Zeit., v. 1, p. 342 (1908). - Id., Berlin. Ent. Zeit., v. 52 (1907), p. 165 (1908). - Id. Tierreich, Lief. 25, p. 78 (1909). - Fruhstorfer: A. Seitz, Großschmett. Erde, v. 5, p. 299 (1912). 
? O. quirinus, F. D. Godman \& O. Salvin: Whymper, Travels Great Andes, App., 99 (1892)

e re bus Röb. O. e., Röber, Int. Ent. Zeit., Typ.: Peru v. 21, p. 98 (1927).

subsp. bo livianus Stich. o. q. b., 10. q. Bolivia

var. $b$. Staudinger MS), H. Stichel, Berlin.

Ent. Zeit., v. 46 (1901), p. 512 (1902). -

Id., Gen. Ins., Fasc. 20, p. 20 (1904). -

Fruhstorfer, Int. Ent. Zeit., v. 1, p. 31 (1907). - H. Stichel, Berlin. Ent. Zeit., v. 52 (1907), p. 165 (1908). - Id., Tierreich, Lief. 25, p. 76 (1909). - Truhstorfer, A. Seitz, Großschmett. Erde, v. 5, p. 299 (1912). - ? O. b., P. Köhler, Fauna Argent., pars 1 (Zeit. wiss. Ins.-Biol., v. 18, Beiheft), p. 21 (1923).

o. quiteria, Weymer (\& Maassen), Lep. Reise Stübel, p. 100 (cit. part.) (1890).

subsp. meridional is Stauding. O. q. var. Espirito Santo m., O. Staudinger (\& Schatz), Exot. -Santa Schmett., v. 1, p. 213 (1887). - O. m., Catharina, Bönninghauscn, Verb. Ver. Naturw. Un- Paraguay terh. Hamburg, v. 9 (1894-95), p. 39 (1898). - o. q. m., H. Stichel, Berlin. Ent. Zeit., v. 46 (1901), p. 511 (1902). - Id., Gen. Ins., Fasc. 20, p. 20 (1904). - Fruhstorfer, Int. Ent. Zeit., v. 1, p. 30 (1907). H. Stichel, Berlin. Ent. Zeit., v. 52 (1907), p. 167 (part.) (1908). - Id., Tierreich, Lief. 25, p. 76 (part.) (1909). - Fruhstorfer: A. Seitz, Großschmett. Erde, v. 5, p. 299 (non t. $61 \mathrm{c}:=0$. invirae remoliatus) (1912). - O. m., Hild. Schultz, Deutsch. Int. Zeit., p. 26, t. 9, f. 88 (Morphol.) (1914). - o. quiteria m., F. Hoffmann, Zeit. wiss. Ins.-Biol., v. 25, p. 94 (Metamorph.) (1930).

Pavonia quinteria (!), Burmeister, Rev. Mag. Zool., ser. 3, v. 1, p. 42, t. 6, f. 4 (larva) (1873).

? O. cassiae (non Pap. c. Linné), Loquay, Int. Ent. Zeit., v. 22, p. 156 (1928).

forma $p h i l o n$ Fruhst. $O$. q. p., Fruhstor- Typ.:

fer, Int. Ent. Zeit., จ. 1, p. 29, 30, f. 1 Espirito

(O) (1907). - Id., Ent. Zeit. Stettin, v. Santo 68 , p. $134(1907)$. - Id., A. Seitz, Großschmett. Ende, v. 5, t. 61c; p. 299 (1911 \& 12). - o. quiteria - $p$., Zikán, Ent. Rundschau, v. 45 , p. 10 (1928).

o. q. meridionalis, H. Stichel, Tierreich, Lief. 25, p. 76 (in cit.) (1909).

forma ores $b$ ios Fruhst. O. q. o., Fruhstor- Typ.: fer: A. Seitz, Großschmett. Erde, v. 5, p. Sao Paulo 300 (1912).

- badius Stich. $0 . b_{\text {., }} \mathrm{H}$. Stichel, Berlin. Ent. Zeit., Colombia v. 46 (1901), p. 513, t. 9, f. 4 (1902). O. quiteria b., id., Gen. Ins., Fasc. 20, p. 20 (1904). - id., H. Stichel, Soc. Ent., v. 22, p. 
99 (1907). - Fruhstorfer, Int. Ent. Zeit., v. 1 , p. $30(1907)$ - - H. Stichel, Tierreich, Lief. 25, p. 79 (cit. part.) (1909). - 0. b., Fruhstorfer: A. Seitz, Großschmett. Erde, v. 5, p. 300 (1912). - Fassl, Ent. Rundschau, v. 35, p. 31 (1918).

forma c а и са Röb. O. b. var. c., Röber, Soc. Typ.: Ent., v. 21, p. 20 (1906).

o. $q u$ ite $r i a b$ a $i$ us, H. Stichel, Tierreich, Lief. 25, p. 79 (in cit.) (1909). (? subsp. piopr.)

- camena Stauding. O. c., O. Staudinger (\& Schatz), Colombia Exot. Schmett., v. 1, t. $72 ;$ p. 213 (1886 \& 87). - H. Stichel, Berlin. Ent. Zeit., v. 46 (1901), p. 514 (1902). - Id., Gen. Ins., Fasc. 20, p. 20 (1904). - Id., Tierreich, Lief. 25, p. 82 (1909). - Tassl, Soc. Ent., v. 24, p. 116 ( $\%$, , B, Biol.) (1909). Id., 1. c., v. 27, p. 54 (1912). - Truhstorfer: A. Seitz, Großschmett. Erde, v. 5, p. 300 (1912). - Fassl, Ent. Rundschau, v. 33, p. 44 (1914). - Id., 1. c., v. 31, p. 44 (1916)

- Sallei Doubl. O. s., E. Doubleday (\& Westwood), Venezuela, Gen. Diurn. Lep., t. 57 (1S49). - H. Sti- Colombiachel, Berlin. Ent. Zeit., v. 46 (1901), p. 513 Bolivia, (1902). - Id., Gen. Ins., Fasc. 20, p. 20 ? Mittel(1904). - Id., Tierreich, Lief. 25, p. 79 Amerika (1909). - Fruhstorfer: A. Seitz, Großschmett. Irrde, v. 5, p. 301 (1912).

subsp. Sa llei Doubl. O. 8., E. Doubleday, Venezuela, ut antea, t. 57, f. 2 (1849); J. O. Westwood, Colombia, 1. c., v. 2, p 346 (1851). - Herrich-Schäf- ? Honduras, fer, Corr.-Bl. Zool.-Min. Ver. Regensb., v. Mexiko 19 , p. 64 (1865). - ? Boisduval, Consid. Lép. Guatemala, p. 81 (1870). - W. F. Kirby, Cat. Diurn. Lep., p. 126 (1871). $\frac{1}{107}$ (1879). - Chr. Aurivillius, Svenska Ak. Handl., n. ser., v. 19, Nr. 5, p. 76 (1882). - O. Staudinger (\& Schatz), Exot. Schmett., v. 1 p. 213 (1887). - Weymer (\& Maasson), Lep. Reise Stübel, p. $74(1890)$. - 0 . s. s., H. Stichel, Berlin. Ent. Zeit., v. 46 (1901), p. 514 (1902). - Id., Gen. Ins., Fase. 20, p. 20 (1904). - O. \&., Röber, Soc. Ent., v. 21, p. 27 (1906). - 0. s. s., H. Stichel, Soc. Ent., v. 22, p. 100 (1907). Id., Tierreich, Lief. 25, p. 81 (1909). Fruhstorfer: A. Seitz, Großschmett. Erde, v. 5, p. 301 (1912). - 0. s., Fassl, Soc. Ent., v. 27, p. 54 (ovum) (1912). - Id., Eut. Rundschau, v. 22, p. 100 (1918).

subsp. $m u t a t u s$ Stich. O. s. m., H. Stichel, Ecuador, Berlin. Ent. Zeit, v. 46 (1901), p. 514, f. Nord-Peru 2 (1902). - Id., Gen. Ins., Fasc. 20, p. 20 (1904). - Röber, Soc. Ent., v. 21, p. 27 (1906). - II. Stichel, 1. c., v. 22, p. 100 (1907). - Id., Tierreich, Lief. 25, p. 
81, f. 20 (1909). - Fruhstorfer: A. Seitz, Großschmett. Erde, v. 5, p. 301 (1912).

subsp. farrago Stich. O. s. f., H. Stichel, Süd-Peru, Gen. Ins., Fasc. 20, p. 20 (1904). - Id., Bolivia Tierreich, Lief. 25, p. 82 (1909). - Fruhstorfer: A. Seitz, Großschmett. Erde, v. 5, p. 301 (1912).

forma $n i$ c and $r u s$ Fruhst. $O . \quad s . n$., Fruh- Typ.: storfer: A. Seitz, Großsschmett. Erde, v. Bolivia 5, p. 301 (1912).

(Coroico)

- invirae (Hübn.) Brassolis i., Jac. Hübner, Verz. Guayana, Schmett., p. 50 (1818). - Opsiphanes $i ., \mathrm{H}$. Stichel, Berlin. Ent. Zeit., v. 46 (1901), p. 514 (1902). - Id., Gen. Ins., Fasc. 20, p. 21 (1904). - Id., Tierreich, Lief. 25, p. 83 (1909). - Fruhstorfer: A. Seitz, Großschmett. Erdo, v. 5, p. 301 (1912).

VenezuelaRio Grande do Sul und Paraguay. Honduras

subsp. invirae (Hübn.) ,Potamis superba Guayana, $i^{\prime \prime \prime}$, Jac. Hübner, Samml. Exot. Schmett., v. Venezuela, 1, t. [76], f. 1, 2 (1806-19). - Brassolis Nordi., id., ut antea (1818). - Id., Index Exot. Lep., p. 4 (1821). - Id., Samml. Exot. Schmett., v. 2 , t. [66], f. 3,4 (Q) (182226). - Opsiphanes i., (E. Doubleday \&) J. O. Westwood, Gen. Diurn. Lep., v. 2, p. 346 (1851). - O. \& R. Fielder, Wien. Ent. Monatschr., v. 6, p. 122 (1862). - Herrich-Schäffer, Corr.-Bl. Zool.-Min. Ver. Regensb., v. 19, p. 64 (1865). - W. F. Kirby, Cat. Diurn. Lep., p. 127 (1871). Id., Cat. Doll. Diurn. Lep. Hewitson, p. 107 (part.) (1879). - F. D. Godman \& O. Salvin, Biol. Centr.-Amer., Lep.-Rhop., v. 1, p. 127 (part.) (1881). - Chr. Aurivillius, Svenska Ak. Handl., n. ser., v. 19, Nr. 5, p. 76 (1882). - O. Staudinger ( $\&$ Schatz), Exot. Schmett., v. 1, p. 214 (1887). - o. cassiae var. i., A. Seitz, Ent. Zeit. Stettin, v. 51, p. 30 (1890). - Weymer, ibid., v. 55, p. 322 (1894). - ,Potamis Superba $i . ", W$. F. Kirby (sec. Hübner), Jac. Hübner \& Geyer, Samml. Exot. Schmett., Neue Ausg., v. 1, t. 76 (1894-95). - Brassolis i., id., 1. c., v. 2, t. 279 (66); Opsiphanes i., v. 3, p. 52 (1898 \& 1901). Opsiphanes $i, i$, H. Stichel, Berlin. Ent. Zeit., v. 46 (1901), p. 514 (part.) (1902). - O. i., W. J. Kaye, Trans. City London Ent. Nat. Hist. Soc., 1902, p. 55 (1903). o. i. i., H. Stichel, Gen. Ins., Fase. 20, p. 21 (part.) (1904). - Fruhstorfer, Int. Ent. Zeit., v. 1, p. 30 (1907). - H. Stichel, Tierreich, Lief. 25, p. 85 (1909). - Fruhstorfer: A. Seitz, Großschmett. Erde, v. 5, p. 301 (1912). - O. i., Schwanwitsch, Proc. Zool. Soc. London, p. 521, 522 (Morphol.) (1924). 
subsp. cus pidatus Stich. O. i. c., H. Sti- Panama,

chel, Gen. Ins., Fase. 20, p. 21 (1904). - Costa Rica

Fruhstorfer, Int. Ent. Zeit., v. 1, p. 30 (1907). - H. Stichel, Berlin. Ent. Zeit., v. 52 (1907), p. 169 (1908). - Id., Tierreich, Lief. 25, p. 86, f. 21 (1909). - Fruhstorfer: A. Seitz, Großschmett. Erde, v. 5, p. 301 (1912).

o. invirae i., H. Stichel, Berlin. Ent. Zeit., v. 46 (1901), p. 515 (part.) (1902).

? o. i., H. G. Dyar, Proc. U. S. Nat. Mus!, v. 47 , p. 144 (1914).

subsp. relucens Fruhst. 0 . i. r., Fruh- Honduras storfer, Ent. Zeit. Stettin, v. 68, p. 130 (1907). - Id., Int. Ent. Zeit., v. 1, p. 30 (1907). - H. Stichel, Berlin. Ent. Zeit., v. 52 (1907), p. 163 (1908). - Id., Tierreich, Lief. 25, p. 88 (1909). - Fruhstorfer: A. Scitz, Großschmett. Erde, v. 5, p. 301. (1912).

subsp. Stich eli Röb. O. s., Röber, Soc. Ent., Colombia v. 21 , p. 20 (1906). - O. invirae s., H. Stichel, Soc. Ent., v. 22, p. 98 (1907). Id., Berlin. Ent. Zeit., v. 52, p. 164 (1908). - Id., Tierreich, Lief. 25, p. 86 (1909). Fruhstorfer: A. Scitz, Großschmett. Erde, v. 5, p. 302 (1912).

O. i. cuspidatus (part.) +, O. i. i., Fruhstorfer, Ent. Zeit. Stettin, v. 68, p. 139 (1907).

O. intermedius (non Stich.), Fassl, Ent. Rundschau, v. 35, p. 31 (1918).

subsp. intermedius (Stich.) 0 . invirae Westintermedius (O. inv. var. intermedia Staudinger MS), H. Stichel, Berlin. Ent. Zeit., v. 46 (1901), p. 515 (1902). - H. Stichel, Gen. Ins., Fase. 20, p. 21 (1904). - Fruhstorfer, Int. Ent. Zeit., v. 1, p. 30 (1907). - H. Stichcl, Tierreich, Liet. 25, p. 88 (1909). - Truhstorfer: A. Seitz, Großschmett. Erde, v. 5, p. 302 (1912).

? O. in viirae, W. F. Kirby (sec. Hewitson), Cat. Coll. Diurn. Lep. Hewitson, p. 107 (part.) (1879).

subsp. ledon Fruhst. 0 . i. l., Fruhstorfer: OstA. Seitz, Großschmett. Erde, v. 5, p. 302 Amazonas (1912).

subsp. a gast the nes Fruhst. O. i. a., Fruh- Südstorfer, Ent. Zeit. Stettin, v. 68, p. 132 Peru (? p. 138: "androsthenes") (1907). Id., Int. Ent. Zeit., v. 1, p. 30 (1907). H. Stichel, Int. Ent. Zeit., v. 1, p. 342 (1908). - O. i. amplificatus forma agasthenes, id., Berlin. Ent. Zeit., v. 52 (1907), p. 165 (1908). - Id., Tierreich, Lief. 25, p. 88 (1909). - O. i. agasthenes, Fruhstorfer: A. Seitz, Großschmett. Erde, v. 5, p. 302 (1912). - O. i. amplificatus forma 
agasthenes, Strand, Soc. Ent., v. 33, p. 19 (1918).

forma rectifasciata Fruhst. O. i. Typ.: agasthenes forma r., Fruhstorfer, Ent. Zeit. Pozuzzo Stettin, v. 68, p. 133 (1907). - H. Stichel, Int. Ent. Zeit., v. 1, p. 342 (1908). - O. i. amplificatus forma $r_{\text {., }}$ id., Berlin. Ent. Zeit., ช. 52 (1907), p. 166 (1908). Id., Tierreich, Lief. 25, p. 88 (1909). o. i. r., Fruhstorfer: A. Scitz, Großschmett. Erde, v. 5, p. 302 (1912).

suhsp. is a goras Fruhst. O. invirae isa-Bolivia goras, Fruhstorfer, Ent. Zeit. Stettin, v. (Cocha68, p. 138 (1907). - Id., Int. Ent. Zeit., bamba) v. 1, p. $30(1907)$. - O. invirae amplificatus forma isagoras, H. Stichel, Tierreich, Lief. 25, p. 87 (in cit.) (1909). - 0 . invirae isagoras, Fruhstorfer: $\Lambda$. Seitz, Großschmett. Erde, v. 5, t. 61 c; p. 302 (1911 \& 12).

O. invirae amplificatus, H. Stichel, Berlin. Ent. Zeit., v. 52 (1907), p. 164 (in cit.) (1908).

subsp. remoliat us Fruhst. O. i. r., Fruh- Espirito Santo, storfer, Ent. Zeit. Stettiu, v. 68, p. 131 Mato Grosso(1907). - o. $i$. remuliata (!), id., Int. Rio Grande Ent. Zeit., v. 1, p. 30 (1907). - O. i. amplificatus forma remoliata, H. Stichel, Berlin. Ent. Zeit., v. 52 (1907), p. 165 (1908). - Id., Tierreieh, Lief. 25, p. 88 (1909). o. i. r., Fruhstorfer: A. Seitz, Großschmett. Einde, v. 5, t. $61 \mathrm{c}\left(\sigma^{7}\right)$, t. $61 \mathrm{c}$ (O: falso meridionalis); p. 302, 1131, 1140 (1911, $12 \& 24$ ). - W. Rotlischild, Nov.Zool., v. 23, p. 311 , t. 6 , f. 10,11 (larva, chrysal.) (1916). - Talbot, Bull. Hill Mus., จ. 2, p. 199 (1928).

?. O. invirae (cassiae var.), A. Seitz, Ent. Zeit. Stettin, v. 51, p. $30(1890)$. - O. i., Weymer, 1. e., v. 55, p. 322 (1895). Mabilde, Guia prat. Lep. Rio Gr. do Sul, p. 91 (not. hiol.) (1896). - Silva, Relat. Contrib. Hist. Nat. Lep. Brasil (Congr. Seient. Lat.-Amer., 1905, v. 3B), p. 95, f. 60 (ㅇ) (1907)

forma pseudophilon Fruhst. 0 . i. p., Typ.:

Fruhstorfer, Int. Ent. Zeit., v. 1, p. 29, 30, Espirito f. 2 (1907). - Id., Ent. Zeit. Stettin, v. Santo 68, p. 135 (1907). - Id.: A. Seitz, Großschmett. Ende, v. 5, t. $61 \mathrm{~d}$ (\%); p. 302 (1911\& \& 12).

o. i. amplificatus, H. Stichel, Tlierreich, Lief. 25, p. 87 (in eit.) (1909).

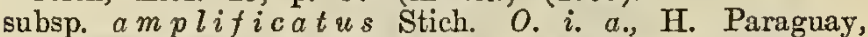
Stichel, Gen. Ins., Fasc. 20, p. 21 (part.) Argentinien (1904). - Fruhstorfer, Int. Ent. Zeit., v. 1, p. 30 (1907). - H. Stichel, 1. c., v. 1, p. 342 (sine synon.) (1908). - Id., Berlin. Ent. Zeit., v. 52, (1907), p. 165 (forma 
principalis) (1908). - Id., Tierreich, Lief. 25 , p. 87 (forma principalis) (cit. part.) (1909). - Fruhstorfer: A. Seitz, Großschmett. Erde, v. 5, p. 302 (1912). ? Strand, Soc. Ent., 33, p. 19 (1918). Hayward, Ent. Record Journ. Var., v. 41, p. 34, 35 (larva, chrysal.) (1929).

o. in virae i. H. Stichel, Berlin. Ent. Zeit., v. 46 (1901), p. 514 (part.) (1902).

o. quiteria meridionalis (non Staudinger), H. Stichel, Berlin. Ent. Zeit., v. 46, p. 511 (part.); v. 52, (1907), p. 163 (correct.) (1902 \& 1908).

- cassina Feld. O. c., C. \& R. Felder, Wien. Ent. MexikoMonatschr., v. 6, p. 122 (1862). - H. Peru, Stichel, Berlin. Ent. Zeit., v. 46 (1901), p. Guayana, 515 (1902). - Id., Gen. Ins., Fasc. 20, p. Amazonas, 21 (1904). - Id., Tierreich, Lief. 25, p. ? Bolivia 88 (1909). - Fruhstorfer: A. Seitz, Großschmett. Ende, v. 5, p. 302 (1912).

subsp. c a s sina Feld. O. c., O. \& R. Felder, Westut antea (1862). - Herrich-Schäffer, Corr.B1. Zool.-Min. Ver. Regensb., v. 19, p. 64 (1865). - W. F. Kirby, Cat. Diurn. Lep., p. 126 (1871). - O. Staudinger (\& Schatz), Exot. Schmett., v. 1, p. 214 (1887). o. c. e., H. Stichel, Berlin. Ent. Zeit., v, 46 (1901), p. 516, f. 3 (1902). - Id., Gen. Ins., Fasc. 20, p. 21 (1904). - Id., Tierreich, Licf. 25, p. 90 , f. 22 (1909). Fruhstorfer: A. Seitz, Großschmett. Erde, v. 5 , p. 302 (1912).

O. cassiae (non Pap. c. Linné), W. F. Kirby, Cat. Diurn. Lep., Suppl., p. 716 $(1877)$. - O.c., O. c. Viar., O. Staudinger (\& Schatz), Exot. Schmett., v. 1, p. 214; t. 71 (forma trans. ad subsp. periphetes) (1887 \& 86).

0. Fabricii (non Caligo f., Boisduval), Chr. Aurivillius, Svenska Ak. Handl., n. ser., v. $19, \mathrm{Nr} .5$, p. 73 (part. in cit.) (1882).

subsp. M e ri a n a e Stich. - Merian, Ins. Suri- Guayana, nam, t. 32 (1705) (conf. Burmeister, Abh. Na- ? Venezuela. turf. Ges. Halle, v. 2, 1854, Sitz-Ber., p. Trinidad, 63: Pavonia cassiae). - Merian-Buchoz, Hist. ? Cuba Ins. Surinam, v. 1, p. 32 , t. $32 ;$ v. 2, p. 49: "Cassiae" (1771). - O. cassina m., H. Stichel, Berlin. Ent. Zeit., v. 46 (1901), p. 518 (1902). - Id., Gen. Ins., Fasc. 20, p. 21 , t. 3, f. 4 (1904). - Id., Tierreich, Lief. 25, p. 91 (1909). - Fruhstorfer: A. Seitz, Großschmett. Erde, v. 5, p. 303 (1912). - W. J. Kaye, Trans. Ent. Soc. London, p. 548 (1904).

Papilio (Danaus) cassiae, Linné, Syst. Nat., ed. 10, p. 471 (part. in cit.) (1758). P. c., J. C. Fabricius, Syst. Ent., p. 483 
(part. in cit.) (1775). - "Cassiae", Papilio c., P. Cramer, Pap. Exot., v. 2, p. 14, t. 106, f. A: p. 148 (1777). - F. (Danaus) c., Goeze, Ent. Beytr., v. 3. I, p. 193 (part. in cit.) (1779). - P. c., J. O. Fabricius, Spec. Ins., v. 2, p. 58 (part. in cit.) (1781). O. Stoll, Essai Syst. Lep. (P. Cramer, Pap. Exot., v. 4, App.), p. 8, Nr. 22 (part.) (1782). - J. d. Fabricius, Mant. Ins., v. 2, p. 28 (part. in cit.) (1787). - $P$. (Danaus) c., Gmelin-Linné, Syst. Nat., ed. 13, v. 1. V, p. 2280 (part. in cit.) (1790). $P$. c., Jung, Alphab. Verz. Schmett., v. 1, p. 106 (part. in cit.) (1791). - J. C. Fabricius, Ent. syst., v. 3. I, p. 150 (part. in cit.) (1793) - (Jablonsky \&) Herbst, Naturs. Ins., Schmett., v. 6, p. 54 (part.), t. 129 , f. 3 (non f. 1,2 ) (1793). - Turton, Gen. Syst. Nat. Linné, v. 3. IT, p. 117 (1806). - P. c., Brassolis, J. C. Fabricius, MS: Illiger, Mag. Ins.kunde, v. 6, p. 282 (1807). - P. c., G. Shaw, Natural Miscell., v. 19, t. 791 (MLtam., fig. mal.) Index (1807). - Gravenhorst, Vergl. Ubers. Zool. Syst., p. 313 (part.) (1807). - Brassolis c., Jac. Hübner, Verz. Schmctt., p. 50 (1818). - Morpho c., Pavonia c., (Iatreille \&) J. B. Godart, Enc. Méth., v. 9, p. 454 (part.), p. 807 (1824). - Pavonia c., Verloren, Cat. Ins. Lep. Oramer, v. 1, p. 50; v. 2, p. 202 (1837). - E. Blanchard, Iiist. An. Artic., v. 3, p. 454 (part. in cit.) (1840). - E. Doubleday, List Lep. Brit. Mus., v. 1, p. 118 (1844). - Opsiphanes c., (E. Doubleday \&) J. O. Wcstwood, Gen. Diurn. Lep., v. 2, p. 346 (part. in cit.) (1851). - ? Opsiphanes c., H. Lucas: Sagra, Hist. fis. Cuba, v. 7, p. 249 (1857). - C. \& R. Felder, Wien. Ent. Monatschr., v. 6, p. 123 (1862). - Herrich-Schäffer, Corr.-Bl. Zool.-Min. Ver. Regensb., v. 19, p. 64 (1865). - A. G. Butler, Cat. Diurn. Lep. Fabr., p. 41 (cit. Fabricii part.) (1869). - W. F. Kirby, Cat. Diurn. Lep., p. 126, 646; Suppl., p. 716 (part.) (1871 \& 77). Möschler, Verh. Zool.-Bot. Ges. Wien, v. 26, Abh., p. 321 (cit. part.) (1877). ? Caracciola, Ann. Soc. Int. France, ser. 6, v. 9, p. OLXXVIII (1889). - W. F. Kirby, Samml. Exot. Selhmett. Hübner \& Geyer, v. 3, p. 51 (1901). - W. J. Kaye, Trans. City London Ent. Nat. Hist. Soc., 1902, p. 55 (1903).

Ca ligo Fabricii, Boisduval, Consid. Lép. Guatemala, p. 54 (part. in cit.) (1870). Opsiphanes f., Ohr. Aurivillius, Svenska Ak. Handl., n. ser., v. 19 , 'Nr. 5, p. 73 (cit. part.) (1882). - W. J. Kaye, Trans. Ent. Soc. London, p. 165 (1904). 
subsp. $n u m a t i u s$ Fruhst. O. c. n., Fruh- Colombia storfer: A. Seitz, Großschmett. Erde, v. 5, p. 303 (1912). - Jurriaanse, Tijdschr. Ent., v. 66, p. 148 (Physiol.) (1923).

subsp. periphetes Fruhst. O. c. p., Fruh-Colombia storfer: A. Seitz, Großschmett. Erde, v. 5, p. 303 (1912). - Jurriaanse, Tijdschr. Ent., v. 66, p. 148 (Physiol.) (1923). (Subsp. dub., ? forma praeced.)

O. c $a$ s sina, Fassl, Ent. Rundschau, v. 35, p. 31 (1918).

subsp. chiriquensis Stich. O. cassina chi- Panama riquensis, H. Stichel, Berlin. Ent. Zeit., v. (Chiriqui) 46 (1901), p. 517 (1902). - Id., Gen. Ins., Fasc. 20, p. 21 (1904). - Id., Tierreich, Lief. 25 , p. 93 (1909). - Fruhstorfer: A. Seitz, Großschmett. Erde, v. 5, p. 303 (1912).

O. cas sine var, F. D. Godman \& O. Salvin, Biol. Centr.-Amer., Lep.-Rhop., v. 2, p. 664 (1901).

subsp. Fabrici $i$ (Boisd.) Caligo f., Bois- Panamaduval, Consid. Lép. Guatemala, p. 54 (sine Mexiko cit.) (1870). - Opsiphanes f., Chr. Aurivillius, Svenska Ak. Handl., n. ser., v. 19, $\mathrm{Nr} . \quad 5$, p. 73 (cit. part.) (1882). - 0 . cassina f., H. Stichel, Berlin. Ent. Zeit., v. 46 (1901), p. 516 (1902), - Id., Gen. Ins., Fasc. 20, p. 21 (1904). - Id., Tierreich, Lief. 25, p. 93 , f. 23 (cit. nonnull. except.) (1909). - Iruhstorfer: A. Seitz, Großschmett. Erde, v, 5, t. $61 \mathrm{~d}$ (o', ㅇ); p. $302(1911 \& 12)$. - Gibbs, Proc. Ent. Soc. London, p. XIVIII (1912).

O. cassiae (non Pap. c. Linné), A. G. Butler \& Herb. Druce, Proc. Zool. Soc. London, p. 339 (cit. part.) (1874). - F. D. Godman \& O. Salvin, Biol. Centr.-Amer., Lep.-Phop., v. 1, p. 127 ; จ. 2, p. 664 (part.) (1881 \& 1901).

? O. Crameri (non Felder), F. M. Fountaine, Entomol., v. 46, p. 194 (not. biol.) (1913).

subsp. a equ a torialis Stich. O. c. a., H. OstStichel, Berlin. Ent. Zeit., v. 46 (1901), Ecuador p. 517 , t. 9 , f. 5 (1902). - Id., Gen. Ins., Fase. 20, p. 21, t. 3, f. 5 (1904). - Id., Tierreich, Lief. 25, p. 91 (1909). - Fruhstorfer: A. Seitz, Großschmett. Erde, v. 5, p. 303 (1912).

subsp. notandus Stich. O. c. notanda, H. Peru,

Stíchel, Ins.-Börse, จ. 21, p. 21 (1904). O. c. notandus, id., Gen. Ins., Fasc. 20 , p. 21 (1904). - Id., Tiemeich, Lief. 25, p. 94 (1909). - Fruhstorfer: A. Seitz, Großschmett., $\nabla .5$, p. 303 (1912).

O. c $a$ s sina merian a e (non Stich.), Strand, Soc. Ent., v. 33, p. 19 (1918). 
? O. Fabricii (non Caligo f. Boisd.), P. Köhler, Fauna Argent., pars 1 (Zeit. wiss. 15

Ins.-Biol., v. 18, Beiheft), p. 21 (1923).

Species dubia.

lutescentefasciatus (Goeze) Kirby Seba, Thesaurus, $\nabla .4$, p. 20 , t. 15 , f. 1,2 (1765). - Papilio (Danaus) lutesconte-fasciatus, Goeze, Int. Beytr., v. 3. I, p. 222 (1779). - Opsiphanes lutescentefasciatus, W. F. Kirby, Cat. Diurn. Lep., Suppl., p. 716 (1877). - H. Stichel, Berlin. Ent. Zeit., v. 46 (1901), p. 519 (1902). - Id., Tierreich, Lief. 25, p. 94 (1909).

\section{Genus Opopfera Chr. Auriv.}

Opoptcra (Subgenus), Chr. Aurivillius, Svenska Ak. Handl., n. ser., v. 19 , Nr. 5, p. 75 (1882). - O. (Genus), H. Stichel. Berlin. Ent. Zeit., v. 46 (1901), p. 488 (1902). - Id., Gen. Ins., Fasc. 20, p. 22, t. 2, f. 1 (Morphol.) (1904). - Id., Tierreich, Lief. 25, p. 94, f. 24 (Morphol.). (1909). - O. (Artengruppe), Fruhstorfer: A. Seitz, Großschmett. Erde, v. 5, p. 291, 292 (1912). O., Strand, Soc. Ent., v. 33, p. 19 (1918). - Schwanwitsch, Proc. Zool. Soc. Lond., p. 520, 521 (Morphol.) (1924). - Le Cerf, Enc. Ent., ser. B. 3, Lep., v. 1, p. 141 (1926).

C a lig o, Jac. Hübner, Samml. Exot. Schmett., v. 2, t. [71] $(1822-26)$. - C. (part.), E. Doubleday, List Lep. Brit. Mus., v. 1, p. 117, 118 (1844). - (E. Doubleday \&) J. O. Westwood, Gen. Diurn. Lep., v. 2, p. 340 (1851). *Herrich-Schäffer, Corr.-Bl. Zool.-Min. Ver. Regensb., v. 19 , p. 65 (1851). - W. F. Kirby, Cat. Diurn. Lep., p. 128 (1871). - Id., Samml. Exot. Schmett. Hübner \& Geyer, Neue Ausg., v. 2, t. 284 (71) (1894-95).

Morpho (part.), Latreille (\& Godart), Enc. Méth., v. 9, p. 435 ; J. B. Godart, 1. e., p. 447 ete. (1824).

$P$ a vonia (part.), (Latreille \&) J. B. Godart, Enc. Méth., v. 9, p. 807 (1824). - "Pavonie", Pavonia, Guérin-Méneville, Iconogr. Règne Anim., v. 2, p. 487; Ins., t. 79 (182938). - Pavonia (part.), Griffith-Cuvier, Anim. Kingdom, v. 15, p. 590 (1832). - Boisduval, Spec. Gén. Lép., v. 1, Expl. Pl., p. 4; t. 12 (1836). - E. Doubleday (\& TVestwood), Gen. Diurn. Lep., t. 54 (1849). - Burmeister, Rev. Mag. Zool., ser. 3, v. 1, p. 43 (part.) (1873).

Opsiphanes (part.), (E. Doubleday \&) J. O. Westwood, Gen. Diurn. Lep., v. 2, p. 344 (1851). - Ohenu \& H. Lucas, Enc. Hist. Nat., Pap. Diurn., p. 169 (1851). Ménétriés, Enum. Corp. Anim. Ac. Petropol., pars 1, p. 38 pars 2, p. 38 (1855 \& 57). - Prittwitz, Ent. Zeit. Stettin, v. 26, p. 309 (1865). - Herrich-Sohäffer, Corr.-Bl. Zool.Min. Ver. Regensb., v. 19, p. $64(1865)$. - W. F. Kirby, Cat. Dium. Lep., p. 125; Suppl., p. 716 (1871 \& 77). Hopffer, Ent. Zeit. Stettin, v. 35, p. 358 (1874). - W. F. Kirby, Entomologist, v. 11, p. 26 (part.). - Id., Cat. Coll. Diurn. Lep. Hewitson, p. 107 (1879). - O. Staudinger (\& Schatz), Exot. Schmett., v. 1, p. 211 (1887). - L. 
Glaser, Cat. Etymol. Col. Lep., p. 304, 383 (1887). F. D. Godman \& O. Salvin, Ann. Mag. Nat. Hist., ser. 6, v. 14, p. 95 (1894). - Bönninghausen, Verh. Ver. Naturw. Unterh. Hamburg, v. 9 (1894-95), p. 38 (1896). - W. F. Kirby, Samml. Exot. Schmett. Hübner \& Geyer, Neue Ausg., v. 3, p. 50 (1901). - F. D. Godman \& O Salvin, Biol. Centr.-Amer. Lep.-Rhop., v. 2, p. 665 (1901). Fruhstorfer: A. Seitz, Grolsschmett. Arde, v. 5, p. 291, 292 (1912). - W. Rothschild, Nov. Zool., v. 23, p. 310 (1916). - P. Köhler, Tauna Argent., pars 1 (Zoit. wiss. Ins.-Biol., v. 18, Beiheft), p. 21 (1923). - Zikán, Ent. Rundschau, v. 45 , p. 10 (1928).

Eryphanis (part.), W. F. Kirby, Cat. Diurn. Lep., App., p. 646 (1871).

\section{Sectio Desmidocosmefi}

H. Stichel, Gen. Ins., Fasc. 20, p. 23 (1904). - Id., Tierreich, Lief. 25 , p. 96 (1909).

\section{a. Cohors Aorsiformes}

H. Stichel, Gen. Ins., Fasc. 20, p. 23 (1904). - Id., Tierreich, Lief. 25, p. 96 (1909).

aorsa (God.) Morpho a., Pavonia o., (Latreille \&) Nord- bis J. B. Godart, Enc. Móth., v. 9, p. 447, 807 Süd(1824). - Opoptera a., H. Stichel, Berlin. Brasilien, Fnt. Zeit., v. 46 (1901), p. 489 (1902). - Colombia, Id., Gen. Ins., Fasc. 20, p. 23 (1904). - Ecuador, Id., Tierreich, Lief. 25, p. 96 (1909). - Peru Opsiphanes a., Fruhstorfer: A. Seitz, Großschmett. Erde, v. 5, p. 292 (1912).

subsp. a orsa (God.) Morpho a., Pavonia a., Espirito Santo J. B. Godart, ut antea (1824). - P. a., E. -Rio Grande Doubleday, List Lop. Brit. Mus., v. 1, p. 118 do Sul (1844). - E. Doubleday (\& Westwood), Gen. Diurn. Lep., t. 54, f. 3 (1849). Opsiphanes a., J. O. Westwood, 1. c., v. 2, p. 345 (1851) - O a., Herrich-Schüffer, Corr.Bl. Zool.-Min. Ver. Regensb., v. 19, p. 64 (1865). - W. F. Kirby, Cat. Diurn. Lep., p. 126 (1871). - Id., Cat. Coll. Diurn. Lep. Hewitson, p. 107 (1879). - Opsiphanes (Opoptera) a., Chr. Aurivillius, Svenska Ak. Handl., n. ser., v. 19, Nr. 5, p. 75 (1882). - Ops. a., O. Staudinger (\& Schatz), Exot. Schmett., v. 1, p. 212 (1887). - Mabilde, Guia prat. Lep. Rio Gr. do Sul, p. 90, t. 8, f. 2 (1896). - Opoptera a. a., H. Stichel, Berlin. Ent. Zeit., v. 46 (1901), p. 489 (1902). - Id., Gen. Ins., Fase. 20, p. 23 (1904). - Fruhstorfer, Ent. Zeit. Stettin, v. 86, p. 144 (1907). - H. Stichel, Tierreich, Lief. 25, p. 98 (forma principalis) (1909). - Opsiphanes a. a., Fruhstorfer: A. Seitz, Großschmett. Erde, v. 5, t. 63 a; p. 292 (1912). 
forma $l$ it $u$ ra Fruhst. $0 . a . \quad l$., Fruhstorfer, Typ.: Ent. Zeit. Stettin, v. 68, p. 144 (1907). - Espirito O. a. a. forma $l$., H. Stichel, Berlin. Ent. Santo Zeit., v. 52 (1907), p. 172 (1908). - Id., Tierreich, Lief. 25, p. 99 (1909). - Opsiphanes a. ab. l., Fruhstorfer: A. Seitz, Großschmett. Erde, v. 5, p. 292 (1912). Opsiph. aorsa-l., Zilkán, Ent. Rundschau, v. 45, p. 10 (1928).

subsp. hilara Stich. O. a. h., H. Stichel, OstBerlin. Ent. Zeitschr., v. 46 (1901), Ecuador, p. 498 (1902). - Id., Gen. Ins., Fasc. Peru 20, p. 23 (1904). - Id., Tierreich, Lief. 25, p. 99, f. 25 (1909). - Opsiphanes a. h., Fruhstorfer: A. Seitz, Großschmett. Erde, v. 5, p. 292 (1912).

subsp. $f u s$ c a ta Stich. 0. a. f. (Ops. aorsina WestStauding. MS), H. Stichel, Berlin. Ent. Zeit., v. 52 (1907), p. 175 (1908). - Id., Tierreich, Lief. 25, p. 99 (1909). - Opsiphanes Amazonas a. h., Fruhstorfer: A. Seitz, Großsehmett. Erde, v. 5, p. 292 (1912).

subsp. colonbiana (Rothsch.) Opsiphanes Colombia a. c., W. Rothschild, Nov. Zool., v. 23, p. 310 (1916).

arsippe (Hopff.) Opsiphanes a., Hopffer, Ent. Zeit. Peru, Stettin, v. 35, p. 358 (1874). - Opoptera Bolivia a., H. Stichel, Berlin. Ent. Zeit., v. 46 (1901), p. 490 (1902). - Id., Gen. Ins., Fasc. 20, p. 23 (1904). - Id., Tierroich, Lief. 25, p. 100 (1909). - Opsiphanes a., Fruhstorfer: A. Seitz, Großschmett. Irrdo, v. 5, p. 292 (1912).

subsp. arsippe (Hopff.) Oppsiphanes a., Süd-Peru Hopffer, ut antea (1874). - W. F. Kirby, Cat. Diurn. Lep., Suppl., p. 716 (1877). - Id., Cat. Coll. Diurn. Lep. Hewitson, p. 107 (1879). - 0. (Opoptera) a., Chr. Aurivillius, Srenska $\Lambda \mathrm{k}$. Handl., n. ser., v. 19, Nr. 5, p. 75 (1882). - Ops. a., O. Staudinger (\& Schatz), Exot. Schmett., v. 1, t. 71 ; p. 212 (1886 \& 87). - Opoptera a. a., Stichel, Berlin. Ent. Zcit., v. 46 (1901), p. 490 (1902). - Id., Gen. Ins., Fasc. 20, p. 23 (1904). - Id., Tierreich, Lief. 25, p. 101 (1909). - Opsiphanes a. a., Fruhstorfer: A. Seitz, Großschmett. Erde, v. 5, p. 292 (1912). - Opoptera a. a., Strand, Soc. Ent., v. 33, p. 19 (1918).

subsp. bracteolata Stich. O. a. b., Ir. Bolivia Stichel, Berlin. Ent. Zcit., v. 46 (1901), (La Paz) p. 490 (1902). - Id., Gen. Ins., Fasc. 20, p. 24, t. 4, f. $3(1904)$. - Id., Tierreich, Lief. 25, p. 102 (1909). - Opsiphanes a. b., Fruhstorfer: A. Seitz, Großschmett. Erde, v. 5, p. 293 (1912). - Opoptera a. b., Biedermann, Enc. Ent., ser. B. 3, Lep., v. 3, p. 1, t. 2, $f_{\text {f }}$ $7,8\left(0^{7}\right)$; t. 3, f. 13,14 (q) (1928). 


\section{b. Cohors Symiformes}

H. Stichel, Gen. Ins., Fasc. 20, p. 24 (1904). - Id., Tierreich, Lief. 25, p. 102 (1909).

Fruhstorferi (Röb.) Opsiphanes f., Röber, Ent. Santa

Nachr., v. 22, p. 323 (1896). - Opoptera f., Catharina

H. Stichel, Berlin. Ent. Zeit., v. 46 (1901), (Lages)

p. 490 (1902). - O. $f_{\text {., }}$ (Opsiphanes sosius

Stauding. MS), H. Stichel, Gen. Ins., Fasc. 20 , p. 24 (1904). - Opoptera f., id., Tierreich, Lief. 25, p. 102 (1909). - Opsiphanes

f., Frubstorfer: A. Seitz, Großschmett. Frde, v. 5 , p. 330, t. 63a (1912).

Opsiphanes sulcius (part.), O. Staudinger (\& Schatz), Exot. Schmett., v. 1, p. 212 () (1887).

sulcius (Stauding.) Opsiphanes s. (part.), O. Stau- Sao Paulodinger (\& Schatz), Exot. Schmett., v. 1, p. Rio Grande $212\left(\sigma^{\top}\right)$ (1887). - Opoptera s., H. Stichel, do Sul Berlin. Ent. Zeit., v. 46 (1901), p. 490 (1902). - Id., Gen. Ins., Fasc. 20, p. 24, t. 4, f. 4 (1904). - Id., Tierreich, Lief. 25, p. 103 (forma principalis) (1909). - Opsiphanes s., Fruhstorfer: A. Seitz, Großschmett. Erde, v. 5, p. 293, t. 63 a (1912).

forma sodalis (Röb.) Opsiphanes sulcius Typ.: var. sodalis, Röber, Soc. Ent., v. 20, p. 177 Sao Paulo (1906). - Opoptera sulcius forma sodalis, H. Stichel, Tierreich, Lief. 25, p. 104 (1909). - Opsiphanes, sulcius "Torm" sod., Fruhstorfer: A. Seitz, Großschmett. Erde, v. 5, p. 292 (1912).

syme (Hübu.) Caligo s., Jac. Hübner, Samml. Exot. Mittel-, Schmett., v. 2, t. [71], f. 3, 4 (1822-26). - SüdOpoptera s., H. Stichel, Berlin. Ent. Zeit., v. 46 (1901), p. 491 (1902). - Td., Gen. Ins., Fasc. 20, p. 24 (1901). - Id., Tierreich, Lief. 25, p. 105 (1909). - Opsiphanes s., Fruhstorfer: A. Seitz, Grolschmett. Eide, v. 5 , p. 293 (1912).

subsp. sy me (Hübn.) Caligo s., Jac. Hübner, Espirito Santo ut antea, f. 3, 4 (1822-26). - Pavonia s., - Santa Boisduval, Spoc. Gén. Lép., v. 1, Expl. Pl., Catharina, p. 4; t. 12, f. 2 (1836). - Caligo s., Argentinien F. Doubleday, List Lep. Brit. Mus., v. 1, p. (Misiones) 117 (1844). - Opsiphanes s., (E. DoubBrasilien, Argentinien, leday \&) J. O. Westwood, Gien. Diurn. Lep., v. 2, p. 345 (1851). - Chenu \& H. Lucas, Enc. Hist. Nat., Pap. diurn., p. 170 (1851-53). - Prittwitz, Ent. Zeit. Stettin, v. 26, p. 309 (1865). - Herrich-Schäffer, Corr.-B1. Zool.-Min. Ver. Regensb., v. 19, p. 64 (1865). - W. F. Kirby, Cat. Diurn. Lep., p. 125 (1871). - Pavonia s., Burmeister, Rev. Mag. Zool., ser. 3, v. 1, p. 43 (1873). - Opsiphanes s., W. F. Kirby, Entomologist, v. 11, p. 26 (1878). - Cat. 
Coll. Diurn. Lep. Hewitson, p. 107 (1879). - o. (Opoptera) s., Chr. Aurivillius, Svenska Ak. Handl., n. ser., v. 19, Nr. 5, p. 75 (1882). - Opsiph. s., O. Staudinger (\& Schatz), Exot. Schmett., t. 71 ; p. 212 (1886 \& 87). - L. Glaser, Cat. Etymol. Col. Lep., p. 304,383 (1887). - N. Reuter, Acta Soc. Sci. Fenn., v. 22, p. 112 (1896). Bönninghausen, Verh. Ver. Naturw. Unterh. Hamburg, v. 9 (1894-95), p. 38 (1896). - Caligo s., Opsiphanes s., W. F. Kirby, Samml. Fxot. Schmett. Hübner \& Geyer, Neue Ausg., v. 2, t. 284 (71); v. 3, p. $50(1898 \& 1901)$. - Opoptera 8., $H$. Stichel, Berlin. Ent. Zeit., v. 46 (1901), p. 491 (1902). - 0. 8. s., id., Gen. Ins., Fasc. 20 , p. 24 (1904). - 0. 8., Id., Tierreich, Lief. 25, p. 105 (forma principalis) (1909). - Opsiphanos 8., Fruhstorfer: A. Seitz, Großschmett. Erde, v. 5, p. 293 (non t. 63 b: = forma fumosa) (1912). - P. Köhler, Fauna Argent., pars 1 (Zeit. wiss. Ins.Biol., v. 18, Beiheft), p. 21 (1923). Zikán, Ent. Rundschau, v. 45, p. 10 (1928).

alcimedon (Dalm.) Papilio a., Dalman, Analecta Ent., p. 41 (1823). - Caligo a., (E. Doubleday \&) J. O. Westwood, Gen. Diurn. Lep., v. 2, p. 342 (1851). - Herrich-Schäffer, Corr.-Bl. Zool.-Min. Ver. Regensb., จ. 19, p. 65 (1865).

acadina (God.) Morpho a., Pavonia a., (Latreille \&) J. B. Godart, Enc. Méth., v. 9, p. 451, 807 (1824). - P. a., Guérin-Méneville, Iconogr. Règne Anim,, v. 2, Ins., t. 79, f. 1 ; v. 3, p. 487 (1829-44), - Griffith-Cuvier, Anim. Kingdom, v. 15 , t. 47, f. 1 (1832). - Opsiphanes a., Ménétriés, Enum. Corp. Anim. Ac. Petropol., Lep., pars 1, p. 38 (1855). - Caligo a., Eryphanis a., W. F. Kirby, Cat. Diurn. Lep., p. 128; App., p. 646 (1871). - Opoptera a., Schwanwitsch, Proc. Zool. Soc. London, p. 520, 522, t. 3, f. 15 (Morphol.) (1924).

forma $f u$ mos a Stich. 0 . s. $f .$, H. Stichel, Typ.: Berlin. Ent. Zeit., v. 46 (1901), p. 24 Espirito (1902). - Id., Gen. Ins., Fasc. 20, p. 24 Santo (1904). - o. s. forma $f .$, id., Berlin. Ent. Zeit., v. 52 (1907), p. 278 (1908). - Id., Tierreich, Lief, 25, p. 106 (1909). - Opsiphanes s. f., Fruhstorfer: A. Seitz, Großschmett. Erde, v. 5, p. 292 (1912).

opoptera syme (non Caligo s. Hübn.), Fruhstorfer: A. Seitz, Großschmett. Erde, v. 5, t. $63 \mathrm{~b}$; p. 1140 (corr.) (1912).

subsp. colombicola (Rothsch.) Opsipha- Colombia nes s. c., Rothschild, Nov. Zool., v. 23, p. (Popoyan) 310 (1916). 


\section{Sectio Peragnosfi}

H. Stichel, Gen. Ins., Fasc. 20, p. 24 (1904). - Id., Tierreich, Lief. 25, p. 106 (1909).

Staudingeri (Godm. \& Salv.) Opsiphrnes s., F. D. Panama, Godman \& O. Salvin, Ann. Mag. Nat. Hist., Costa Rica ser. 6, v. 14, p. 95 (1894). - Tid., Biol.Centr. Amer, Lep.-Rhop., v. 2, p. 665, t. 107, f. 17 (1901). - Opoptera s., H. Stichel, Berlin. Ent. Zeit., v. 46 (1901), p. 491 (1902). - Td., Gen. Ins., Fasc. 20, p. 24 (1904). - Id., Berlin. Ent. Zeit., v. 52 (1907), p. 176 (1908). - Id., Tierreich, Lief. 25 , p. 107 , f. 26 (1909). - Opsiphanes s., Fruhstorfer: Großschmett. Erde, v. 6 5 , p. 293 , t. 63 b (1912).

\section{Genus Cafoblepia H. Stich.}

II. Stichel, Berlin. Ent. Zoit., v. 46, p. 491 (1902). - Id., Gen. Ins., Fasc. 20, p. 25, t. 2, f. 2 (Morphol.) (1904). $\neg$ Catoblepsia (crr. typ!), Catoblepia, Id., Soc. Ent., v. 22, p. 92 (1907). - Id., Ticrrcich, Lief. 25, p. 108, f. 27 (Morphol.) (1909). - Catoblepia (Artengruppe), Fruhstorfer: A. Seitz, Großschmett. Erde, v. 5, p. 291, 304 (1912). - C., Strand, Lep. Niepeltiana, v. 1, p. 37; v. 2, p. 12 (1914 \& 16). - Id., Soc. Ent., v. 33, p. 19 (1918).

Dan a us [Subgenus] (part.), Linné, Syst. Nat., ed. 10, p. 468, 471 (1758). - Linné-Houttyn, Natuurl. Hist., v. 1. XI, p. $278(1766-69)$. - Linné, Syst. Nat, ed. 12, v. 1. II, p. 758, $767(1767)$ - - D. (Festivus) (part.), D. H. Schnoider, Nomencl. Entom., p. 35 (1785). - D. (part.), GmelinLinné, Syst. Nat., ed. 13, v. 1. V, p. 2230, 2232 (1790).

„P ot a mis superba", Jac. IIübner, Samml. Exot. Schmett., v. 1, t. $73(1806-19)$. - „P. S.", W. F. Kirby, L. c., Neue Ausg., p. 73 (1894-95).

Bras soli is (part.), Jac. Hübner, Verz. Schmett., p. 50 (1818). - Id., Samml. Exot. Schmett., v. 2, t. [67] (1820 -26). - Td., Index Exot. Lep., p. 2 (1821). - W. F. Kirby, Samml. Exot. Schmett. Hübner \& Goyer, Neue Ausg., v. 2, t. 280 (67) (1898).

Morpho (part.), Latreille (\& Godart), Enc. Mroth., v. 9, p. 435 ; J. B. Godart, 1. c., p. 453 (1824).

P a vo nia (part.), (Latreille \&) J. B. Godart, Enc. Méth., v. 9, p. 807 (1824). - Lacordaire, Ann. Soc. Ent. France, v. 2, p. 396 (1833). - Verloren, Cat. Ins. Lep. Cramer, v. 2, p. 202 (1837). - 'P. ? (part.), E. Doubleday, List Lep. Brit. Mus., pars 1, p. 118 (1844). - P. (part.), Burmeister, Rev. Mag. Zool., ser. 3, v. 1, p. 43 (1873). - Id., Deser. Phys. Argent., v. 5. I, p. 314 (1878).

Opsiphanes (part.), (E. Doubleday \&) J. O. Westwood, Gen. Diurn. Lep., v. 2, p. 344 (1851). - Chenu \& H. Iucas, Enc. Hist. Nat., Pap. Diurn., p. 169 (1851-53). - Ménétriés, Enum. Corp. Anim. Ac. Petropol., Lep., pars 1, p. 38; pars 2, p. 77 (1855 \& 57). - Herrich-Schäffer, Corr.-Bl. Zool.-Min. Ver. Regensb., v. 19, p. 64 (1865). - A. G. Butler, Cat. Diurn. Lep. Fabr., p. 40 (1869). - O., Hewitson, Ent. Mag., v. 6, p. 177 (1870). - O. (part.), W. F. 
Kirby, Cat. Diurn. Lep., p. 125, 646; Suppl., p. 716 (1871 \& 77). - Hewitson, Exot. Butt., v. 4 (5), t. [7] Opsiphanes (1873). - W. F. Kirby, Trans. Ent. Soc., London, p. 143 (1874). - Herb. Druce, Proc. Zool. Soc. London, p. 217 (1876). - Möschler, Ver. Zool.-Bot. Ges. Wien, v. 26, Abh., p. 321 (1877). - W. F. Kirby, Cat. Coll. Diurn. Lop. Fabr., p. 107 (1879). - F. D. Godman \& O. Salvin, Biol. Centr.Amer., Lep.-Rhop., v. 1, p. 125; v. 2, p. 665 (18.81 \& 1901). - Chr. Aurivillius, Svenska Ak. Handl., n. ser., v. 19, Nr. 5 , p. 74, 75, 76 (1882). - O. Staudinger (\& Schatz), Exot. Schmett., v. 1, p. 211 (1887). - L. Glaser, Cat. Etymol. Col. Lep., p. 358, 387 (1887). - Weymer (\& Maassen), Lep. Reise Stübel, p. 100 (1890). - Mahnel, Deutsch. Ent. Zeit. Lep. (Tris), v. 3, p. 249 (1890). - Bönninghausen, Verh. Ver. Naturw. Unterh. Hamburg, v. 9 (1894-95), p. 38 (1896). - W. F. Kirby, Samml. Exot. Schmett. Hübner \& Gever, Neue Ausg., v. 3, p. 50 (1901). - Silva, Relat. Contrib. Hist. Nat. Lep. Brasil (Congr. Scient. Lat.-Amer., 1905 , v. 3 B) p. 94 (1907). - A. G. Weeks, Ill. Diurn. Lep., v. 2, p. XI (1911). - Fruhstorfer: A. Seitz, Großsclimett. Erde, v. 5, p. 291, 304 (1912). - W. Rothschild, Nov. Zool., v. 23, p. 311, 312 (1916). - Fassl, Ent. Rundschau, v. 33 , p. 26 (1916). - Fruhstorfer, 1. c., v. 34, p. 56 (1917). - Zikán, l. c., จ. 45, p. 10 (1928).

C a ligo (part.), Boisduval, Consid. Lép. Guatemala, p. 54 $(1870)$.

\section{Sectio Dyophthalmi}

H. Stichel, Gen. Ins., Fasc. 20, p. 26 (1904). - Id., Tierreich, Lief. 25, p. 111 (1909). - Fruhstorfer: A. Seitz, Großschmett. Erde, v. 5, p. 304 (1912).

xanthus (L.) Papilio (Danaus) x., Linné, Syst. Nat., Guayana, ed. 10 , p. $472(1758)$. - Catoblepia $x ., H$. Nord-

Stichel, Berlin. Fnt. Zeit., จ. 46 (1901), p. Brasilien, 492 (1902). - Id., Gen. Ins., Fasc. 20, p. 26 (1904). - Id., Tierreich, Lief. 25, p. 111 (1909). - Opsiphanes x., Fruhstorfer: A. Seitz, Großschmett. Erde, v. 5, p. 304 (1912). - Catoblepia $x$., Niepelt: Strand, Lep. NieOstColombia, Ecuador, Peru, pelt. I. p. 37 (1914).

subsp. $x$ anthus (L.) - ? Seba, Thesaurus, v. 4 , p. 20 , t. 15 , f. $5,6(1765)$. - Petiver, Opera Hist. Nat., Gazophyll. Nat. Art., t. 136, f. 1, 2 (1767). - Papilio (Danaus) $x$., Linné, ut antea (1758). - P. x., id., Mus. Ludov. Ulr., p. 267 (1764). - P. (Danaus) $x$., Linné, Syst. Nat., ed. 12, v. 1. ПI, p. 767 (1767). - Linné-Houttyn, Natuurl. Hist., v. 1. XI, p. 278 (1767). - P. x., P. I. S. Müller, Naturs. Linné, v. 5. I, p. 598 (1774). - J. O. Fabricius, Syst. Ent., p. 483 (cit. part.) (1775). - (P. F. Gmelin \&) Christman, Onomatol. Med., $\mathrm{v} .6$, p. 162 (1775).

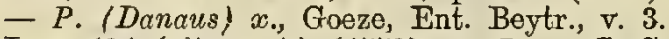
I, p. 194 (cit. part.) (1779). - P. x., J. O. Fabricius, Spec. Ins., V. 2, p. 58 (cit. part.) (1781). - P. (Danaus Festivus) x., D. H. 
Schneider, Nomencl. Entom., p. 35 (1785). $P$. $x$. J. O. Fabricius, Mant. Ins., v. 2, p. 28 (cit. part.) (1787). - P. (Danaus) $x$. , Gmelin-Tinné, Syst. Nat., ed. 13, v. 5. I, p. 2282 (cit. part.) (1790). - P, x., Jung, Alphab. Verz. Schmett., v. 2, p. 281 (1792). J. C. Fabricius, Ent. syst., v. 3. I, p. 150 (cit. part.); Index alphab., p. 129 (1793 \& 96). - Fr. IVeber, Nomencl. Entom., p. 101 (1795). $-P . x ., \alpha$, Thunberg, Mus. Nat. Ac. Upsal., pars 23, p. 9 (1804). - $P$. x., Turtou, Gen. Syst. Nat. Linné, v. 3. II, p. 117 (1806). - Brassolis xanthis (!), Jac. Hübner, Verz. Schmett., p. 50 (1818). - Morpho xanthus, Prvonia x., (Latreille \&) J. B. Godart, Enc. Méth., v. 9, p. 453, 807 (1824). $P$. x., Tatreille, Aun. Soc. Ent. Franoe, v. 2, p. 396 (1838). - P. ? x., E. Doubleday, List Lep. Brit. Mus., v. 1, p. 118 (1844). - Opsiphanes x., (E. Doubleday \&) J. O. Westwoorl, Gen. Diurn. Lep., v. 2, p. 345 (cit. part.) (1851). - Chenu \& H. Lucas, Enc. Mist. Nat., Pap. Diurn., p. 170 (1851). - Ménétriés, Enum. Corp. Ac. Petropol., Lep., pars 1, p. $3 \mathrm{~S}$ (sine cit.) (1855). Herrich-Schäffer, Corr.-Bl. Zool.-Min. Ver. Regensb., v. 19, p. 64 (1865). - A. G. Butler, Cat. Diurn. Lop. Fabr., p. 40 (part.) (1869). - Papilio x. (Opsiphanes), W. T. Kirby, Trans. Ent. Soc. London, p. 143 (recogn. typ. Linnéi) (1870). - Id., Cat. Diurn. Lep., p. 126; Suppl., p. 716 (part.) (1871 \& 77). - Id., Cat. Coll. Diurn. Lep. Hewitson, p. 107 (1879). - ? Möschlor, Verh. Zool.Bot. Ges. Wien, v. 26, Abh., p. 321 (sine cit.) (1877). - Chr. Aurivillius, Svenska Ak. Handl., n. ser., v. 19, Nr. 5, p. 74 (cit. part.); p. 75 (1882). - O. Staudinger (\& Schatz), Exot. Schmett., v. 1, p. 213 (1887). - L. Glaser, Cat. Etymol. Col. Lep., p. 387 (1887). - W. F. Kirby, Samml. Exot. Schmett. Huibner \& Geyer, Neue Ausg., v. 3, p. 51 (part.) (1901). Catoblepia $x . x_{0}, \mathrm{H}$. Stichel, Berlin. Ent. Zeit., v. 46 (1901), p. 492 (1902). - Id., Gen. Ins., Fasc. 20, p. 26 (1904). - Id., Tierreich, Lief. 25, p. 112 (cit. corrig.), f. 28 (1909). - Opsiphanes $x$. x., Fruhstorfer: $\Lambda$. Seitz, Großschmett. Ende, v. 5, p. 304 , t. 63 b (1912).

Brassolis quiteria (non Papilio q. Cramer), Jac. Huibner, Verz. Schmett., p. 50 (in cit.) (1818). - Weymer (\& Maassen), Lep. Reise Stübel, p. 100 (part. in cit.) (1890).

? Opsiphanes amphirhoe (non Brassolis a. Hübner), A. G. Weeks, M1. Diurn. Lep., v. 2, p. XI (1911). 
subsp. soranus (Westw.) Opsiphanes s., (E. Amazonas, Doubleday \&) J. O. Westwood, Gen. Diurn. NordostLep., v. 2, p. 345 (1851). - Herrich- Peru

Schäffer, Corr.-Bl. Zool.-Min. Ver. Regensb., v. 19 , p. $64(1865)$. - W. F. Kirby, Cat Diurn. Lep., p. 126 (1871). - Catoblepia xanthus s., H. Stichel, Berlin. Ent. Zeit., v. 46 (1901), p. 493 (1902). - Id., Gen. Ins., Fasc. 20, p. 26 (1904). - Id., Tierreich, Lief. 25, p. 113 (cit. part.) (1904). - Opsiphanes x. s., Fruhstorfer: A. Seitz, Großschmett. Erde, v. 5, p. 305 (1912). Catoblepria s., Niepelt: Strand, Lep. Niepelt. I. p. 37 (1914).

Opsiphanes $x$ anthus, Chr. Aurivillius, Svenska Ak. Handl., n. ser., v. 19, Nr. 5, p. 74 (part. in cit.) (1882).

subsp. Dohrni Stich. C. $x$. dohrni, H. Sti- Ostchel, Berlin. Ent. Zeit., v. 46 (1901), p. 493, t. 9, f. 1 (1902). - Id., Ins.-Börse, v. 21, p. 6 (1904). - Id., Gen. Ins., Fasc. 20, p. 26, t. 4, f. 5. (q) (1904). - Id., Tierreich, Lief. 25, p. 114 (cit. part.) (1909). — Opsiphanes x. d., Frubstorfer: A. Seitz, Großschmett. Erde, v. 5, p. 305 , t. 63 b (1912). - Catoblepia $x$. d., Niepelt: Strand, Lep. Niepeltiana, v. 1, p. 37 (1914). - C. d., Fassl, Ent. Rundschau, v. 35, p. 31 (1918).

? Opsiphanes $x a n t h u s$, Herb. Druce, Proc. Zool. Soc. London, p. 217 (1876).

? O. soranus, W. F. Kirby, Cat. Coll. Diurn. Lep. Hewitson, p. 107 (1879).

rivalis Niep. C. r., Niepelt, Deutsch. Ent. Zeit. Iris, Ecuador v. 25, p. 123 (1911). - Fruhstorfer: A. Seitz, Großschmett. Erde, v. 5, p. 306 (1912). - Niopolt: Strand, Lep. Niepeltiana, v. 1, p. 37, t. 9, f. 7 (1914). - C. vivalis (err. typ.), D. Sharp, Zool. Record, v. 48 (1911), pars 12, p. 291 (1912). - Descr. ㅇ: Paulo major quam mas, colore brunnea pallidiore, signaturis mari adaequatis. $\Lambda \mathrm{l}$. ant. long. $58 \mathrm{~mm}$. Typ.: N. O. Peru (Iquitos).

rersitincta Stich. C. v. (Opsiphanes selectus Stau- Guayana ding. MS), H. Stichel, Berlin. Ent. Zeit., v. 46 (1901), p. 495 , t. 9, f. 2 (1902). - Id., Gen. Ins., Fase. 20, p. 27 (1904). - Id., Int. Ent. Zeit. Guben, v. 1, p. 342 (1908). - Id., Tierreich, Lief. 25, p. 115 (1909). Opsiphanes v., Fruhstorfer: A. Seitz, Großschmett. Erde, v. 5, p. 304, t. 66 a (1912).

orgetorix (Hew.) Opsiphanes o., Hewitson, Ent. Panama, Monthly Mag., v. 6, p. 177 (1870). - Cato- Nicaragua, blepia orgetoryx, H. Stichel, Berlin. Ent. Ecuador Zeit., v. 46 (1901), p. 496 (1902). - C. orgetorix, id., Gen. Ins., Fasc. 20, p. 27 (1904). - Id., Tierreich, Lief. 25, p. 116 (1909). - Opsiphanes o., Fruhstorfer: A. Seitz, Großschmett. Erde, v. 5, p. 305 (1912). 
subsp. orgetorix (Hew.) Opsiphanes o., Panama, Hewitson, ut antea (1870). - IV. F. Kirby, Nicaragua Cat. Diurn. Lep., p. 126; Suppl., p. 716 (1871 \& 77). - Hewitson, Exot. Butt., v. 4 (5), t. [7] Opsiphanes 1, f. 1, 2 (9) (1873). - Id., Cat. Coll. Diurn. Lep. Hewitson, p. 107 (1879). - F. D. Godman \& O. Salvin, Biol. Centr.-Amer., Lep.-Rhop., v. 1, p. 129; v. 2, p. 665 (1881 \& 1901). - Chr. Aurivillius, Svenska Ak. Handl., n. ser., v. 19, Nr. 5, p. 76 (1882). - Catoblepia orgetoryx o., H. Stichel, Berlin. Ent. Zeit., v. 46 (1901), p. 496 (1902). - C. orgetorix o., id., Gen. Ins., Fasc. 20, p. 27 (1904). - Id., Tierreich, Lief. 25, p. 117 (1909). - Opsiphanes o. o., Fruhstorfer: A. Seitz, Großschmett. Erde, v. 5, t. 64a; p. 305 (1911 $\& 12)$.

subsp. magnali is Stich. C. orgetoryx m., H. WestStichel, Berlin. Ent. Zeit., v. 46 (1901), p. Ecuador 496 (1902). $-C$. orgetorix m., id., Gen. Ins., Fasc. 20, p. 27 (1904). - Id., Tierreich, Lief. 25, p. 118 (1909). - Opsiphanes o. m., Fruhstorfer: A. Seitz, Großschmett. Erde, v. 5 , p. 305 (1912).

forma Fle $m m$ ing $i$ (Rothsch.) Opsiphanes f., Typ.: W. Rothsehild, Nov. Zool., v. 23, p. 311, t. 5, f. 4 (1916). - O. f., Catoblepia f., H. Stichel, Neue Beitr. syst. Ins.kunde, v. 3, p. 68 (1925).

- amphirhoe (Hübn.) Brassolis a., Jac. Hübner, Samml. Exot. Schmett., v. 2, t. 67 (1822-26). Opsiphanes a., Ménétriés, Enum. Corp. An. Ac. Petropol., Iep., pars 1, p. 38 (in cit. amphiroe) (1855). - Herrich-Schäffer, Corr.Bl. Zool.-Min. Ver. Regensburg, v. 19, p. 64 (1865). - ?? F. D. Godman \& O. Salvin, Biol. Centr.-Amer., Lop.-Rhop., v. 1, p. 129 (1881). - Chr. Aurivillius, Svenska Ak. Handl., n. ser., v. 19, Nr. 5, p. 75, 76 (1882). - O. Staudinger (\& Schatz), Exot. Schmett., v. 1, p. 213 (1887). - Brassolis a., W. F. Kirby, Samml. Exot. Schmett. Hübner \& Geyer, Neue Ausg., v. 2, t. 280 (67) (1898). - Catoblepia a., H. Stichel, Berlin. Ent. Zeit., v. 46 (1901), p. 496 (1902). - Id., Gen. Ins., Fasc. 20, p. 27 (1904). - Catoblepsia (err. typ.!) a., id., Soc. Ent., v. 22, p. 92 (1907). - Catoblepia a., id., Tierreich, Lief. 25 , p. 118 , f. 29 (ㅇ) (1909). - Opsiphanes a. a., Fruhstorfer: A. Seitz, Großschmett. Eide, v. 5, p. 307; t. 64 a (falso placita) (1912 \& 11). - ? Catoblepia a., Strand, Soc. Ent., v. 33, p. 19 (patria falsa?) (1918).

Morpho xanthus, Pavoniax., (non Papilio $x$. Linné), (Latreille \&) J. B. Godart, Enc. Méth., v. 9, p. 453, 807 (part. in cit.) (1824).

Nordwest-

Ecuador

(Rio

Dourango)

Mittel-

und

Süd-

Brasilien,

Paraguay,

Argentinien,

? Guatemala

? Surinam 
- Opsiphanes x., (E. Doubleday \&) J. O. Westwood, Gen. Diurn. Lep., v. 2, p. 345 (part. in cit.) (1851). - W. F. Kirby, Cat. Diurn. Lep., p. 126 (part.) (1871). - Pavonia $x$, Burmeister, Rev. Mag. Zool., ser. 3, v. 1, p. 43 (1873). - Id., Descr. Phys. Argent., v. 5. I, p. 22 (1878). - Opsiphanes $x$., Bönninghausen, Verh. Ver. Naturw. Unterh. Iamburg, จ. 9 (1894-95), p. 38 (1896). - W. F. Kirby, Samml. Fxot. Schmett. Hübner \& Geyer, Neue Ansg., v. 3, p. 51 (cit. part.) (1901). - Silva, Relat. Contrib. Hist. Nat. Lep. Brasil (Congr. Scient. Lat.-Amer., 1905 , v. 3. B) p. 97, t. 18, f. 61 (1907).

? Opsiphanes forma cyparissa (non 0 . xanthicles c. Fruhstorfer'), P. Köhler, Fauna Argent., pars 1 (Zcit. wiss. Ins.-Biol., Beiheft), p. 21 (1923).

forma placita Stich. C. $a$. forma $p$., H. Typ.: Santa Stichel, Ent. Zeit. Guben, v. 20, p. 211 Catharina (1906). - Id, Berlin. Ent. Zeit., v. 52 (1907), p. 178 (1908). - Id., Tierreich, Lief. 25, p. 118 (1909). - Opsiphanes a. p., Fruhstorfer: A. Seitz, Großschmett. Erde, v. 5, p. 307 (non t. 64 a: = forma typ. a.) (1912). - ? O. amphitoe (!) p., Zikán, Lnt. Rundschau, จ. 45, p. 10 (1928).

forma s pintas us (Frulist.) Opsiphanes a. Typ.: s., Truhstorfer, Ent. Rundschau, v. 34, p. 56 Sao Paulo (1917).

singularis (Weym.) Opsiphanes s., Weymer, Deutsch. Guatemala Ent. Zeit. Iris, v. 20 , p. 15, t. 1, f. 3 (1907). - Catoblepia 8., H. Stichel, Berlin. Ent. Zeit., v. 52 (1907), p. 179 (1908). Id., Tierreich, Lief. 25, p. 121 (1909). - Opsiphanes s., Truhstorfer: A. Seitz, Großschmett. Frde, v. 5, p. 307 (1912).

xanthicles (Godm. \& Salv.) Opsiphanes $x$., F. D. Panama

Godman \& O. Salvin, Biol. Centr.-Amer., -Bolivia, Lep.-Rhop., v. 1 , p. 130 (1881). - Cato- Westblepia $x .$, H. Stichel, Gen. Ins., Fasc. 20, Amazonas, p. 26 (1904). - Id., Int. Ent. Zeit., v. 1, Guayana

p. 342 (1908). - Id., Tierreich, Lief. 25, p. 122 (1909). - Opsiphanes $x$., Fruhstorfer: A. Seitz, Großschmett. Ende, v. 5, p. 306 (1912).

subsp. xanthicles (Godm. \& Salv.) Opsiphanes $x$. . F. D. Godman \& O. Salvin, ut antea; $t$. 12 , f. 1,2 (falso 0 . xanthus). (1881). - O. Staudinger (\& Schatz), Exot. Schmett., v. 1, p. 213 (1887). - Catoblepia xanthicles, H. Stichel, Berlin. Ent. Zeit., v. 46 (1901), p. 494 (1902). - C. x. x., id., Gen. Ins., Fasc. 20, p. 26 (1904). - Id., Tierreich, Lief. 25 , p. 123 (nonnull. cit. except.) (1909). - Opsiphanes $x$. x., Fruhstor- 
fer: A. Seitz, Großschmett. Erde, v. 5, p. 306 (1912).

? Caligoberecynthia (non Pap. b. Cramer), Boisduval, Consid. Lép. Guatemala, p. $54(1870)$.

Opsiphanes xanthus (non Papilio $x$. Linné), F. D. Godman \& O. Salvin, Biol. Centr.-Amer., Lep.-Rhop., t. 12, f. 1, 2 (1881).

subsp. cyparis s a Fruhst. C. c., Fruhstor- Colombia, fer, Int. Ent. Zeit., v. 1, p. 36, fig. (1907). Ost- Id., Ent. Zeit. Stettin, v. 68, p. 146 (1907). - C. xanthiclcs c., H. Stichel, Int. Ent. Zeit., v. 1, p. 343 (1908). - Id., BerEcuador, lin. Ent. Zeit., v. 52, (1907), p. 173 (1908). - Id., 'Tierreich, Lief. 25, p. 124 (1909). - Opsiphancs $x$. c., Fruhstorfer: A. Seitz, Großschmett. Erde, v. 5, p. 306, t. 66 a (1912). - Fassl, Ent. Rundschau, v. 33, p. 26 (1916). - Catoblepria c., id., 1. c., v. 35, p. 31 (1918).

subsp. b e lis ar Stich. C. $x$. b., (Opsiphanes Bolivia b. Stauding. MS), H. Stichel, Gen. Ins., Fasc. 20, p. 26 (1904). - Id., Berlin. Ent. Zeit., v. 52 (1907), p. 173 (1908). - Id., Tierreich, Lief. 25, p. 125, f. 30 (1909). Opsiphanes $x$. b., Fruhstorfer: A. Seitz, Großschmett. Erde, v. 5, p. 306 (1912).

subsp. sosigenes (Fruhst.) Opsiphanes $x$. Guayana s., Fruhstorfer, Ent. Rundschau, v. 30, p. 133 (1913).

? Seba, Thesaurus, v. 4, p. 16, t. 11 , f. 8,9 (1765). (sec. Aurivillius, 1882: Opsiphanes invirae, sed errans puto).

$P$ a pilio xa $\operatorname{tic} u s$ (non Linné), J. C. Fabricius, Syst. Ent., p. 483 (part. in cit.). (1775). - "Xanthus", Papilio $x ., \mathrm{P}$. Cramer, Pap. Exot., v. 2, p. 131, t. 183, f. A, B; p. $151(1777)$. $P . x$. J. O. Fabricius, Spec. Ins., v. 2, p. 58 (part. in cit.) (1781). - D. Stoll, Essai Syst. Lep. (P. Cramer), Pap. Exot., v. 4, App.), p. 8, Nr. 27 (1782). - J. C. Fabricius, Mant. Ins., v. 2, p. 28 (part. in cit.) (1787). - Id., Ent. syst., v. 3. I, p. 150 (part. in cit.) (1793). - (Jablonsky \&) Herbst, Naturs. Ins., Schmett., v. 6, p. 48 (cit. part.), t. 127, f. 1, 2 (1793). - Tableau, Enc. Méth., v. 18, t. 26, f. 3 , 3bis (1797). - Morpho x., $\mathrm{Pa}$ vonia $x$. (Latreille \&) J. B. Godart, Enc. Méth., v. 9, p. 453, 807 (part. in cit.) (1824). - Pavonia x., Verloren, Cat. Ins. Lep. Cramer, v. 1, p. 75; v. 2, p. 202 (1837). - Opsiphanes $x$. . (E. Doubleday \&) J. O. Westwood, Gen. Diurn. Lep., v. 2, p. 345 (part. in cit.) (1851). - ? Möschler, Verh. Zool.-Bot. Ges. Wien, v. 26, Abh., p. 321 (1877). - A. G. Butler, Cat. Diurn. Lep. Fabr., p. 41 (part.) (1869). - Chr.

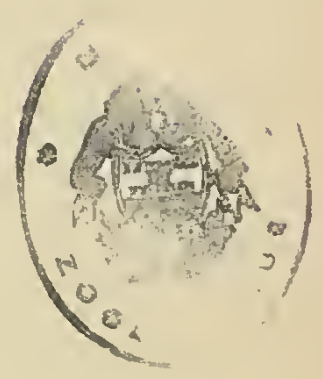


Aurivillius, Svenska Ak. Handl., n. ser., v. 19 , Nr. 5, p. 41 (part. in cit.) (1882).

berecynthia (Cram.) Papilio U., P. Cramer, Pap. Guayana, Exot., v. 2, p. 148 (1777). - Catoblepia b., Vellezuela, II. Stichel, Berlin. Ent. Zeit., v. 46 (1907), Colombia p. 497 (1902). - Id., Gen. Ins., Fase. 20, p. -Bolivia, 27 (1904). - Id., Tierreich, Lief. 25, p. Brasilien 125 (1909). - Opsiphanes b., Fruhstorfer: A. Seitz, Großschmett. Erde, v. 5, p. 307 (1912).

subsp. berecynthia (Cram.) Seba, The- Guayana, saurus, v. 4, p. 15 , t. 11, f. 5, 6 (1765). - Venezuela, „Berecynthia", Papilio b., P. Cramer, ut Amazonas antea, p. 133 , t. 184 , f. B, O (1777). $-P$. (Danaus) b., Goeze, Entom. Beytr., v. 1. II, p. 67 (cit. part.) (1779). - P. b., J. O. Fabricius, Spec. Ins., v. 2, p. 59 (cit. part.) (1781). - C. Stoll, Essai Syst. Lep. Cramer (P. Cramer, Pap. Exot., v. 4, App.), p. 8, Nr. 28 (1782). - P. (Danaus Festivus) berecynthus, [D. H. Schneider], Nomencl. Entom., p. 35 (1785). - P. b., J. O. Fabricius, Mant. Ins., v. 2, p. 298 (cit. part.) (1787). - P. (Danaus) b., Gmelin-Linné, Syst. Nat., ed. 13 , v. 5. I, p. $2280(1790)$. - P. berecynthia, Jung, Alphab. Verz. Schmett., v. 1, p. 75 (1791). - ? C. Stoll, Suppl. P. Cramer, Pap. Exot., p. 16, t. 3, f. 4 A-O (larva, chrysal., fig. dubiae, conf. W. Müller, Zool. Jahrb. Syst., v. 1, p. 594, nota: ? Caligo spec.) (1791) $-P$. berecynthius, (Jablonsky \&) Herbst, Naturs. Ins., Schmett.. v. 6, p. 61, t. 130, f. 2, 3 (1793). - P. berecynthus, J. C. Fabricius, Ent. syst., v. 3. I, p. 151 (cit. part.); Index alphab., p. 119 (1793 \& 96). - Fr. Weber, Nomencl. Entom., p. 101 (1795). - Turton, Gen. Syst. Nat. Linné, v. 3. II, p. 117 (1806). - Gravenhorst, Vergl. Übers. Zool. Syst., p. 313 (1807). - Brassolis berecynthia, Jac. Hübner, Verz. Schmett., p. 50 (part.) (1818). - Id., Index Exot. Lep., p. [2] (part.) (1821). - Morpho berecynthus, Pavonia b., (Latreillo \&) J. B. Godart, Enc. Méth., v. 9, p. 453 (cit. part.), p. 807 (1824). - Pavonia b., Lacordaire, Ann. Soc. Ent. France, v. 2, p. 396 (1833). - Verloren, Cat. Ins. Lep. Oramer, v. 1, p. 76, 152; v. 2, p. 202 (1837). - Pavonia ? b., E. Doubleday, List Lep. Brit. Mus., v. 1, p. 118 (1844). - Opsiphanes b., (E. Doubleday \&) J. O. Westwood, Gen, Diurn. Lep., v. 2, p. 346 (1851). - Ménétriés, Enum. Corp. Anim. Ac. Petropol., Lep., pars 2, p. 77 (1857). - Ierrich-Schäffer, Corr.-Bl. Zool.-Min. Ver. Regensburg, v. 19, p. 64 (1865). - o. berecynthia, A. G. Butler, Cat. Diurn. Lep. Fabr., p. 40 (part.) (1869). - ? Caligo b., Boisduval, Consid. Lép. Guatemala, p. 54 (1870). - Opsiphanes berecyn- 
thus, W. F. Kirby, Cat. Diurn. Lep., p. 126; Suppl., p. 716 (1871 \& 77). - Möschler, Verh. Zool.-Bot. Ges. Wien, v. 26, Abh., p. 321 (1877) - W. F. Kirby, Cat. Coll. Diurn. Lep. Hewitson, p. 107 (part.) (1879). - Chr. Aurivillius, Svenska Ak. Handl., n. ser., v. 19, Nr. 5, p. 76 (1882). - O. Staudinger (\& Schatz), Exot. Schmett., v. 1, p. 212 (1887). - O. berecynthus (-tius), L. Glaser, Cat. Etymol. Col. Liep., p. 358 (1887). - O. berecynthus, Hahnel, Deutsch. Ent. Zeit., Lep. (Tris), v. 3, p. 22 (1890). Catoblepia b. b., H. Stichel, Berlin. Ent. Zeit., v. 46 (1907), p. 497 (1902). - C. berecynthia b., id., Gen. Ins., Fasc. 20, p. 26 (cit. part.) (1904). - Frulustorfer, Ent. Zeit., Stettin, v. 68, p. 145 (1907). - H. Stichel, Berlin. Ent. Zeit., v. 52 (1907), p. 173 (1908). - H. Stichel, Tierroich, Lief. 25 , p. 127 (forma principalis) (cit. part. corrig.) (1909). - Opsiphanes berecynthus, A. G. Weeks, Ill. Diurn. Lep., v. 2, p. XI (1911). - O. berecynthia b., Fruhstorfer: A. Seitz, Großschmett. Ende, v. 5, p. 307 (1912). - Catoblepia berecynthia b., Strand, Soc. Ent., 33, p. 19 (1918). - C. berecynthus, Schwanwitsch, Proc. Zool. Soc. London, p. 521, 522, t. 3, f. 19 (Morphol.) (1924).

oethon (Fabr.) Papilio o., J. C. Fabricius, Spec. Ins., v. 2, p. 59 (1781). - P. (Danaus Festivus) aethon (!), [D. HI. Schneider], Nomencl. Entom., p. 35 (1785). - P. oethon, J. C. Fabricius, Mant. Ins., v. 2, p. 28 (1787) - $-P$. (Danaus) o., Gmelin-Linné, Syst. Nat., ed. 13, v. 1. V, p. 2281 (1790). $P$. o., Jung, Alphab. Verz. Schmett., v. 2, p. 67 (1792). - J. O. Fabricius, Ent. syst., v. 3. I, p. 152; Index alphab., p. 126 (1793 \& 96). - (Jablonsky \&) Herbst, Naturs. Ins., Schmett., v. 6, p. 60 (in cit.) (1793). Morpho o., Pavonia o., (Latreille \&) J. B. Godart, Enc. Méth., v. 9, p. 452, 807 (1824). - Opsiphanes o., (E. Doubleday \&) J. O. Westwood, Gen. Diurn. Iop.s v. 2, p. 346 (1851). - O. aethon, Herrich-Schäffer, Corr.-Bl. Zool.-Min. Ver. Regensb., v. 19, p. 64 (1865). - O. oethon, W. F. Kirby, Cat. Diurn. Lep., p. 127 (1871).

forma latita en $i$ a Frulsst. C. b. forma $l$., Typ.:

Fruhstorfer, Ent. Zeit. Stettin, v. 68, p. 146 Surinam (1907). - II. Stichel, Tierreich, Lief. 25, p. 128 (1909). - Opsiphanes b. l. (aberr.), Fruhstorfer: A. Seitz, Großschmett. Erde, v. 5, t. 64 a; p. 307 (1911 \& 12).

forma v el a ta Stich. C. berecynthus velatus, Typ.:

II. Stichel, Berlin. Ent. Zeit., v. 46 (1901), Surinam p. 498 (1902). - C. berecynthia velata, id., Gen. Ins., Fasc. 20, p. 27 (1909). - C. b. b. 
forma $v .$, Fruhstorfer, Ent. Zeit., Stettin, v. 68, p. 145 (1907). - C. b. velata, H. Stichel, Berlin. Ent. Zeit., v. 52 (1907), p. 173 (1908). - C. b. forma v., id., Tierreich, Lief. 25, p. 128 (1909). - Opsiphanes b. v. (aberr.), Fruhstorfer: A. Seitu, Großschmett. Erde, v. 5, p. 307 (1912).

subsp. vicenciona (Fruhst.) Opsiphanes Ostb. v., Frullstorfer: A. Seitz, Großschmett. Erde, v. 5, p. 308 (1912). - 0. b. var. v., Fassl, Ent. Rundschau, v. 33, p. 26 (1916). Catoblepria v., id., 1. c., v. 35, p. 31 (1918). subsp. $l u x$ uriosa Stich. $C$. berecynthus lu- Colombia xuriosus, H. Stichel, Berlin. Ent. Zeit., v. 46 (1901), p. 497 (1902). - C. bereeynthia luxuriosa, id., Gen. Ins., Fasc. 20, p. 28 (1904). - Id., Tierreich, Lief. 25, p. 129, f. 31 (1909). - Opsiphanes b. l., Fruhstorfer: $\mathbf{A}$. Seitz, Großschmett. Erde, v. 5, p. 308 (1912) subsp. midas Stich. C. b. m., H. Stichel, Soc. Ent., v. 23, p. 83 (1908). - Opsiphanes $b$. m., Fruhstorfer: A. Seitz, Großschmett. Erde, v. 5, p. 308 (1912). - Catoblepia b. m., Strand, Lep. Niepeltiana, v. 2, p. 13 (1916). - Descr. Q: Paulo major quam mas, alarum ant. fascia rufescens multo latior, in cellulam introiens; al. post. fascia distalis (marginalis et submarginalis) ut in mare, sed paulo latior et perfectior. Typ.: Peru merid. (Iquitos). Sunt exempla transientes ad $C . b$. berecynthinam Hopff.

forma macasana Strand C. b. midas forma Typ.: macasana, Strand, Lep. Niepeltiana, v. 2, p. Eeuador 12 , t. 15, f. 9 (1916). - Id., Bull. Soc. (Macas) Zool. France, v. 51, p. 393 (1926).

forma $a n g u$ s $t a$ Strand $C$. $b$. $m$. forma $a$. Typ.: Strand, Lep. Niepeltiana, v. 2, p. 12, t. 15, Ecuador f. 10 (1916). - C. b. m. ab. a., id., Bull. Soa. Zool. France, v. 51, p. 398 (1926).

subsp. berecynthina (Hopff.) Opsiphanes Süd-Peru, b., Hopffer, Ent. Zeit. Stettin, v. 35, p. 358 Bolivia (1874). - W. F. Kirby, Cat. Diurn. Lep., Suppl., p. 716 (1877). - Chr. Aurivillius, Svenska Ak. Handl., n. ser., v. 19, Nr. 5, p. $76(1882)$ - -0 . berecynthus var. berecynthina, O. Staudinger (\& Schatz), Exot. Schmett., v. 1, p. 212 (1887). - Catoblepia berecynthus berecynthina, H. Stichel, Borlin. Ent. Zeit., v. 46 (1901), p. 497 (1902). - C. berecynthia bereeynthina, id., Gen. Ins., Fasc. 20, p. 28 (1904). - Id., Tierreioh, Licf. 25, p. 129 (1909). - Opsiphanes b. b., Fruhstorfer: A. Seitz, Großschmett. Erde, v, 5, t. 64 a, p. 308 (1911 \& 12).

vercingetory $x$ (Stauding.) $0 . v$., 0 . Staudinger (\& Schatz), Exot. Schmett., v. 1, t. $72(1886)$.

subsp. a dje ct a Stich. C. b. a., H. Stichel, Bolivia Ent. Zeit. Guben, v. 20, p. 217 (part.) 
(1906). - Id., Berlin. Ent. Zeit., v. 52 (1907), p. 172 (sine synon.) (1908). - Id., Tierreich, Lief. 25, p. 128 (cit. part.) (1909). - Opsiphaness b. a., Fruhstorfer: A. Seitz, Großschmett. Erde, v. 5, p. 308 (1912).

buenavista (Rothsch.) Opsiphanes berecynthia buenavista, W. Rothschild, Nov. Zool., v. 23 , p. 312 (1916).

subsp. $u n d$ ita $e n$ ia Fruhst. C. b. $u$., Fruh- Rio de storfer, Ent. Zeit. Stettin, v. 68, p. 144 Janeiro, (1907). - Opsiphanes b. u., id., Großschmett. Sao Paulo Ende, v. 5, t. 64 a, p. 308 (1911 \& 1912). "Potamis superba berecyntia", Jac. Hübner, Samml. Exot. Schmett., v. 1, t. $[73]$, f. 1, 2 (1806-19). - Brassolis berecynthia, id., Verz. Schmett., p. 50 (part.) (1818). - Id., Index Lxot. Lep., p. [2] (part.) (1821). - „Potamis Superba berecyntia", W. F. Kirby, Samml. Exot. Schmett., Hübncr \& Geyer, Neue Ausg., t. 73 (1894). - Opsiphanes benecynthus, id., 1. c., v. 3, p. 51 (1901). - Catoblepia berecynthia b., H. Stichel, Gen. Ins., Fasc. 20, p. 27 (part. in cit.) (1904).

Catoblepiaberecynthia adjecta, H. Stichel, Berlin. Ent. Zeit., v. 52 (1907), p. 172 (1908). - Id., Tierreich, Lief. 25, p. 128 (in cit.) (1909)

generosn Stich. C. g., H. Stichel, Berlin. Ent. Zeit., v. 46 , v. $46(1901)$, p. 498, t. 9 , f. 3 (1902). - Id., Gen. Ins., Fasc. 20 , p. 28 (1902). - Id., Tiemeich, Lief. 25, p. 130 (1909). - Fruhstorfer: A. Seitz, Groß9 schmett. Erdre, v. 5, p. 309 (1912).

\section{Genus Selenophanes O. Stauding.}

Sel enophanes (Subgenus), O. Staudinger (\& Schatz), Exot. Schmett., v. 1, p.. 212 (1887). - S. (Genus), H. Stichel, Berlin. Ent. Zeit., v. 46 (1901), p. 499 (1902). - Id., Gen. Ins., Fasc. 20, p. 28, t. 2, f. 3 (Morphol.) (1904). - Id., Soc. Ent., v. 22, p. 92 (1907). - Id., Tierreich, Lief. 25, p. 131, f. 32 (Morphol.) (1909). - S. (Artengruppe), Fruhstorfer: A. Seitz, Großschmett. Erde, v. 5, p. 309 (1912). - S., C. B. Williams, Migr. Butterfl., p. 241 (1930)

Nymphalis [Subgenus] (part.), Goeze, Entom. Beytr., v. 3. 1, p. 226, 286 (1779). - N. (Gemmatus) (part.), [D. H. Schneider], Nomencl. Entom., p. 37 (1785).

Brassolis (part.), Jac. Hübner, Verz. Schmett., p. 50 (1818). - L. Glaser, Cat. Etym. Col. Lep., p. 360 (1887). Morpho (part.), Latreille (\& Godart), Enc. Meth., v. 9, p. 435; J. B. Godart, v. 2, p. 454 (1824).

Pa vo nia (part.), (Latreille \&) J. B. Godart, Enc. Méth., v. 9, p. 807 (1824). - Verloren, Cat. Ins. Lep. Cramer, v. 2, p. 202 (1837).

Opsiphanes (part.), (E. Doubleday \&) J. O. Westwood,

Gen. Diurn. Lep., v. 2, p. 344 (1851). - Ménétriés, Enum. 
Corp. Anim. Ac. Petropol., Lep., pars 1, p. 38 (1855). Herrich-Schräffer, Corr.-BI. Zool.-Min. Ver. Regensb., v. 19, p. $64(1865)$. - W. F. Kirby, Cat. Diurn. Lep., p. 125 (1871). - Möschler, Verh. Zool.-Bot. Ges. Wien, v. 26, Abh., p. 321 (1877). - Wr. F. Kirby, Cat. Coll. Diurn. Lep. Hewitson, p. 107 (1879). - F. D. Godman \& O. Salvin, Biol. Centr.-Amcr., Lep.-Rhop., v. 1, p. 125 (1s81). - Chr. Aurivillius, Svenska Ak. Iandl., n. ser., v. 19, Nr. 5, p. 75 (1882). - O. Staudinger (\& Schatz), Exot. Schmett., v. 1, p. 211 (1887). - L. Glaser, Cat. Etymol. Col. Lep., p. 304, 360 (1887). - Röber: O. Staudinger \& Schatz, Exot. Schmett., v. 2, p. 192 (1889). - A. G. Weeks, Illustr. Diurn. Lep., v. 2, p. XI (1911). - Fruhstorfer: A. Seitz, Großschmett. Erde, v. 5, p. 291, 309 (1912). - W. Rothschild, Nov. Zool., v. 23, p. 312 (1916).

\section{Sectio Nephrochoria}

H. Stichel, Gen. Ins., Fasc. 20, p. 29 (1904). - Id., Tierreich, Lief. 25, p. 133 (1909).

cassiope (Cram.) Papilio c., P. Cramer, Pap. Exot., Nördliches v. 1, p. 152 (1776). - Selenophanes c., H. SüdStichel, Berlin. Ent. Zeit., v. 46 (1901), p. Amcrika 50 (1902). - Id., Gen. Ins., Fasc. 20, p. 29 (1904). - Id., Tierreich, Lief. 25, p. 133 (1909). - Opsiphanes c., Fruhstorfer: A. Seitz, Großschmett. Erde, v. 5, p. 309 (1912). subsp. cassiope (Cram.) „Cassiope", Pa-Guayana, pilio c., P. Cramer, Pap. Exot., v. 1, p. 88, Ostt. 57, f. A, B; p. $152(1775 \& 76)$. - Papilio bis Bolivia (Nymphalis) c., Goeze, Ent. Beytr., v. 3. I, p. 286 (1779). - P. c., C. Stoll, Essai Syst. Lep. (P. Cramer, Pap. Exot,, v. 4, App.), p. 8, Nr. 24 (1782). - P. (Nymphalis Gemmatus) c., [D. H. Schneider], Nomencl. Entom., p. 37 (1785). - P. c., Jung, Alphab. Verz. Schmett., v. 1, p. 107 (1791). - P. cassiopus, (Jablonsky \&) Herbst, Naturs. Ins., Schmett., v. 6, p. 53 (patria falsa), t. 128, f. 3, 4 '(1793). - Brassolis c., Jac. Hü̈bner, Verz. Schmett., p. 506 (1818). - Opsiphanes c., (E. Doubleday \&) J. O. Westwood, Gen. Diurn. Lep., v. 2, p. 345 (1851). - Ménétriés, Enum. Corp. Anim. Ac. Petropol., Lep., pars 1, p. 38 (1855). - HerrichSchäffer, Corr.-B1. Zool.-Min. Ver. Regensb., v. 19, p. $64(1865)$. - W. F. Kirby, Cat. Diurn. Lep., p. 126 (1871). - Pavonia c., Verloren, Cat. Ins. Lep. Oramer, v. 1', p. 35 (1873). - Opsiphanes cassione (!), Möschler, Verl. Zool.-Bot. Ges. Wien, v. 26, Abh., p. 321 (1877). - O. cassiopc, W. F. Kirby, Cat. Coll. Diurn. Lop. Hewitson, p. 107 (? part.) (1879). - Chr. Aurivillius, Svenska Ak. Hand1., n. ser., v. 19, Nr. 5, p. 76 (1882). - O. (Selenophanes) c., O. Staudinger (\& Schatz), Exot. Schmett., v. 1, p. 212 (1887). - O. c., Brassolis c., L. Glaser, Cat. Etymol. Col. Lep., p. 304, 360 (1887). 
- Selenophancs c. c., H. Stichel, Berlin. Ent. Zeit., v. 46 (1901), p. 501 (1902). Id., Gen. Ins., Fasc. 20, p. 29 (1901). - Id., Tierreich, Lief. 25, p. 135 (1909). - Opsiphanes c., Fruhstorfer: A. Seitz, Großschmett. Erde, v. 5, p. 309, t. 66 a (1912). - Selcnophancs c., C. B. Williams, Migi. Butterfl., p. 241 (1930).

caryatis (God.) Morpho c., Pavonia c., (Latreille \&) J. B. Godart, Enc. Méth., v. 9, p. 454, 807 (1824). - P. c., Verloren, Cat. Ins. Lep. Cramer, v. 2, p. 202 (1873).

subsp. cassiopeia (Stauding.) Opsipha- Westnes (S.) c., O. Staudinger (\& Schatz), Exot. Schmett., v. 1, t. 71; p. 211 (1886 \& 87). S. cassiope cassiopeia, H. Stichel, Berlin. Ent. Zeit., v. 46 (1901), p. 501 (1902). Id., Gen. Ins., Fasc. 20, p. 29 (1904). - Id., Tierreich, Lief. 25 , p. 136 (1909). - ? Opsiphanes cassiopeia, A. G. Weeks, Mlustr. Diurn. Lep., $\nabla .2$, p. XI (1911). - O. cassiope cassiopeia, Fruhstorfer: A. Seitu, Großschmett. Erlo, v. 5, p. 309 (1912).

subsp. $a m p l$ ior Stich. S. c. a., H. Stichel, Colombia, Berlin. Ent. Zeit., v. 46 (1901), p. 501 Amazonas (1902). - Id., Gen. Ins., Fasc. 20, p. 29 (als Neben(1904). - Id., Tierreich, Licf. 25, p. 135 (1909). - Opsiphanes c. a., Fruhstorfer: A. form)

Seitz, Großschmett. Erde, v. 5, p. 310 (1912).

? S. cas siopeia, Fassl, Ent. Rundschau, v. 35 , p. 31 (1918).

subsp. a $n d$ rom eda Stich. S. c. a. (Opsi- Bolivia phanes a. Stauding. MS), H. Stichcl, Berlin. Ent. Zeit., v. 46 (1901), p. 501 (1902). Id., Gen. Ins., Fasc. 20 , p. 29 , t. 5 , f. 3 a, b (1904). - Id., Tierreich, Lief. 25, p. 136 (1909). - Opsiphanes a., Fruhstorfer: A. Seitz, Großschmett. Erde, v. 5, p. 310 (1912).

forma $p$ lacentia (Frulst.) Opsiphanes cas- Typ.: siope p., Iruhstorfer: A. Seitz, Großschmett. Coroico Erde, v. 5, p. 310 (1912).

subsp. theogenis Fruhst. S. c. t., Fruh- Mato Grosso storfer, Ent. Zeit., v. 24, p. 193 (1910). Opsiphanes c. t., id.: A. Seitz, Großschmett. Erde, v. 5, p. 310 (1912).

supremus Stich. S. s., H. Stichel, Berlin. Ent. Zeit., Ecuador, v. 46 (1901), p. 501 (1902). - Id., Gen. Peru Ins., Fasc. 20, p. 29 (1909). - Opsiphanes s. Fruhstorfer: A. Seitz, Großschmett. Erde, v. 5, p. 310 (1912).

subsp. $s$ u p re $m u s$ Stich. S. s., H. Stichel, Ecuador ut antea, t. 8 , f. 1 (1902). - S. s. s., id., Gen. Ins., Fasc. 20, p. 29 (1904). - Id., Tierreich, Lief. 25, p. 138 (1909). - Opsiphanes s. s., Fruhstorfer: A. Seitz, Großschmett. Erde, v. 5, p. 310 (1912).

subsp. ditatus Thieme S. s. var. ditatus, Süd-Peru Thieme, Ins.-Börse, v. 19, p. 339 (1902). Id., Berlin. Ent. Zeit., v. 48, Sitzgs.-Ber., 
p. (12) (1903). - S. s. d. H. Stichel, Gen. Ins., Fasc. 20, p. 30 (1904). - Id., Tierreich, Lief. 25, p. 138 (1909). - Opsiphanes s. d., Frubstorfer: A. Seitz, Großschmett. Erde, v. 5, p. 310 (1912).

\section{Sectio Cychlochoria}

H. Stichel, Gen. Ins., Fasc. 20, p. 30 (1904). - Id., Tierneich, Lief. 25, p. 139 (1909).

josephus (Godm. \& Salv.) Opsiphanes j., F. D. Guatemala, Godman \& O. Salvin, Biol. Centr.-Amer., Colombia Lep.-Rhop., v. 1, p. 126 (1881). - Selenophanes $j$., H. Stichel, Berlin. Ent. Zeit., v. 46 (1901), p. 503 (1902). - Id., Gen. Ins., Fasc. 20, p. 30 (1904). - Id., Tierreich, Lief. 25, p. 139 (1909). - Opsiphanes j., Fruhstorfer: A. Seitz, Großsclimett. Erde, v. 5, p. 310 (1912).

subsp. jo se phus (Godm. \& Salv.) Opsipha- Guatemala nes $j$., F. D. Godman \& O. Salvin, ut antea, t. 13, f. 3, 4 (1881). - Ohr. Aurivillius, Svenska Ak. Handl., n. ser., v. 19, Nr. 5, p. 76 (1882). - O. Staudinger (\& Schatz), Exot. Schmett., v. 1, p. 212 (1887). - Röber, 1. c., v. 2, p. 192 (1889). - Selenophanes j. j., H. Stichel, Berlin. Ent. Zeit., v. 46 (1901), p. 503 (1902). - Id., Gen. Ins., Fasc. 20, p. 30 (1904). - Id., Tierreich, Lief. 25, p. 140 (1909). - Opsiphanes $j . j$. ., Frubstorfer: A. Seitr, Großschmett. Erde, v. 5, p. 310 (1912).

subsp. excultus Stich. S. j. e., II. Sti- Colombia chel, Bcrlin. Ent. Zeit., v. 46, (1901), p. 503 (1902). - Id., Gen. Ins., Fasc. 20, p. 30 (1904). - Id., Tierreich, Lief. 25, p. 141, f. 33 (1909). - Opsiphanes j. e., Fruhstorfer: A. Seitz, Großschmett. Erde, v. 5, p. 310, t. 66 a $(1912)$.

subsp. e $x$ c is us (Rothsch.) Opsiphanes $j$. e., WestW. Rothschild, Nov. Zool., v. 23, p. 312 Colombia (1916).

forma te nu if a s ciat a Neust. S. j. t., Neu- Typ.: 3 stetter, Int. Ent. Zeit., v. 22, p. 391 (1929). Rio Dagua

\section{Genus Eryphanis Boisd.}

Eryphanis, Eryphane, Boisduval, Consid. Lép. Guatemala, p. 57, 58 (1870). - Eryphanis, W. F. Kirby, Cat. Diurn. Lep., App., p. 646; Suppl., p. 717 (1871 \& 77). - S. H. Scudder, Fist. Skctch: Proc. Amer. Ac. Sc., v. 10, p. 168 (1875). - W. F. Kirby, Entomologist, v. 11, p. 27 (1878). - Euryphanes, F. D. Godman \& O. Salvin, Trans. Ent. Soc. London, p. 122 (1880). - Eryphanis, iid., Biol. Centr.-Amer., Lep.-Rhop., v. 1, p. 136 (1881): - O. Staudinger (\& Schatz), Exot. Schmett., v. 1, p. 217 (1887). - Röber, 1. a., v. 2, t. 32 (Morphol.); p. 191, 194 (1888 \& 89). - W. F. Kirby, Handb. Lep., v. 1, p. 201 (1894). - H. Stichel, Gen. Ins.; 
Fasc. 20, p. 30 (part.), t. 2, f. 5 (Morphol.) (1904). $\rightarrow$ Id., Tierreich, Lief. 25, p. 141 (part.), f. 34 (Morphol.) (1909). - A. Pagenstecher, Geogr. Verbr. Schmett., p. 413 (1909). - Fruhstorfer: A. Seitz, Großschmett. Erde, v. 5, p. 310 (part.) (1912). - Eryphanes, Fassl, Ent. Rundschau, v. 31, p. 37; v. 33, p. 26; v. 35 , p. 31 (1914, 16 \& 18). - Eryphanis, Strand, Lep. Niepeltiana, v. 2, p. 12 (1916). - Le Cerf, Enc. Ent., ser. B. 3, Lep., v. 1, p. 141 (Anatom.) (1926). - Holdhaus: Chr. Schröder, IIandb. Entom., v. 2, p. 731 (distrib. geogr.) (1927).

Eques (Achivus) [Subgen.] (part.), [D. H. Schneider], Nomencl. Entom., p. 33 (1785). - E. (part.), Gmelin-Linné, Syst. Nat., ed. 13, v. 1. V, p. 2225, 2248-49 (1790).

Mo er a (part.) (nom. praeocc.), Jac. Hübner, Verz. Schmett., p. 51 (1818).

Morpho (part.), Latreille (\& Godart), Enc. Méth., v. 9, p. 435; J. B. Godart, 1. c., p. 447 (1824).

Pa von ia (part.), (Latreille \&) J. B. Godart, Enc. Méth., v. 9, p. 807 (1824). - Lacordaire, Ann. Soc. Ent. France, v. 2, p. 396 (1833). - Verloren, Cat. Ins. Lep. Cramer, v. 2, p. 202 (1837). - E. Doubleday, List Lep. Brit. Mus., v. 1 ; p. 118 (1844). - Pavonia, Caligo, Herrich-Schäffer, Samml. au Bereur. Schmett., Lief. 1, Index; t. [3] f. 3, 4; p. 58 (conf. Dalla Torre, Ent. Nachr.-Bl. Troppau, v. 1, p. 4, 56) (1850 \& 58). $\rightarrow$ C. \& R. Felder, Wien. Ent. Monatschr., v. 6, p. 423 (1.862). - H. W. Bates, Proc. Zool. Soc. London, p. 249 (1863). - Burmaistor, Rev. Mag. Zool., ser. 3, v. 1, p. 41 (1873). - Hewitson, Exot. Butt., v. 4 (5), t. [6], Pavonia 2 (1876). - W. F. Kirby, Cat. Coll. Diurn. Lep. Hewitson, p. 110 (1879).

Pot a $m$ is (part.), Jac. Hübner, Cat. Lép. Coll. Franck, p. 79 (1826).

Opsiphanes (part.), E. Doubleday (\& Testwood), Gen. Diurn. Lep., t. 57 (1849). - W. Müller, Zool. Jahrb. Syst., v. 1, p. 598 (1886).

Caligo (part.) (1. Doubleday \&) J. O. Westwood, Gen. Diurn. Lep., v. 2, p. 342 (1851). - Chenu \& H. Lucas, Enc. Hist. Nat., Pap. Diurn., p. 167 (1851-53). - Ménétriés, Enum. Corp. Anim. Ac. Petropol., Lep., pars 1, p. 38 (1855). - ITerrich-Schäffer, ut antea (1858). - Id., Corr.Bl. Zool.-Min. Ver. Regensb., v. 18, p. 55; v. 19, p. 65 (1864 \& 65). - C. \& R. Felder, Reise Novara, v. 2. II, p. 454 (1866). - A. G. Butler, Cistula Ent., v. 1, p. 3 (1869). - Id., Cat. Dium. Lep. Fabr., p. 39 (1869). - Id., Lep. Exot., p. 29 (1870). - W. F. Kirby, Cat. Diurn. Lep., p. 129; Suppl., p. 717 (1871 \& 77). - H. W. Bates, Cistula Ent., v. 1, p. 74 (1872). - A. G. Butler \& Herb + Druce, Proc. Zool. Soc. London, p. 338 (1874). - Möschler, Verh. Zool.-Bot. Ges. Wien, v. 26, Abh., p. 322 (1877). - L. Glaser, Cat. Etymol. Col. Lep., p. 358 (1887). - Weymer (\& Maassen), Lep. Reise Stübel, p. 62 (1890). - Silva, Relat. Contrib. Hist. Nat. Lep. Brasil (Congr. Scient. Lat.Amer., 1905, v. 3. B) p. 91 (1907).

\section{Sectio Psilocraspeda}

H. Stichel, Gen. Ins., Fasc. 20, p. 31 (1904). - Id., Tierreich, Lief. 25, p. 143 (1909).

- polyxena (Meerb.) Papilio p., (non Cramer, non Mittel-, Denis \& Schifferm.), Meerburgh, Afbeeld. Süd- 
Zeldzaame Gewassen, t. 41 (1775). - Eryphanis p., H. Stichel, Gen. Ins., Fasc. 20, p. 31 (1904). - Id., Tiorreich, Lief. 25, p. 143 (1909). - Fruhstorfer: A. Seitz, Großschmett. Ende, v. 5, p. 311 (1912).

subsp. pol yxena (Mcerb.) Papilio p., Meer- Guayana, burgh, ut antea (1775). - Caligo p., Erypha- Venezuela, nis p., W. F. Kirby, Cat. Diurn. Lep., App., Trinidad, p. 646; Suppl., p. 717 (1871 \& 77). - E. p., Nord-, O. Staudinger (\& Schat\%), Exot. Schmett., v. Mittel1, p. 217 (1887). - E. p. p., H. Stichel, Ins.- Brasilien Börse, v. 21, p. 197 (1904). - Id., Gen. Ins., bis Rio de Fasc. 20, p. 31 (1904). - Id., Tierreich, Lief. 25, p. 145 (1909). - E. p., A. G. Weeks, M1. Diurn. Lep., v. 2, p. XI (1911). - E. p. p., Fruhstorfer: A. Seitz, Großschmott. Erde, v. 5, p. 311, t. 64 b, c (falso automedon, corr. p. 1140) (1912). - E. p., Eltringham, Trans. Ent. Soc. London, p. 367, t. 84 (Anatom.) (1926).

a utomedon (Cram.) "Automedon", Papilio a., P. Cramer, Pap. Exot., v. 1, p. 65, t. 41 , f. $\Lambda, B$; p. $152\left(\sigma^{7}\right) \cdot(1775 \& 76) .-P$. a., J. C. Fabricius, Gen. Ins., p. 253 (1777). - "Artomedon", Meuschen \& Boddaert, Mus. Gronov., p. 70 (1778). - P. a., Goeze, Ent. Beytr., v. 3. I, p. 76 (1779). - J. C. Fabricius, Spec. Ins., v. 2, p. 25 (1781). "Automedon", Pap. a., C. Stoll: P. Cramer, Pap. Ixot., v. 4, p. 204, t. 389 , f. A, B; p. 248 (Q) (1782). - Id., Essai Syst. Lep., l. c., App., p. 8, Nr. 20 (1782). - P. (Eques Achivus) a., [D. II. Schneider'] Nomencl. Entom., p. 33 (1785). - P. a., J. C. Fabricius, Mant. Ins., v. 2, p. 13 (1787). - (Jablonsky \&) Herbst, Naturs. Ins., Schmett., v. 3, p. 84 , t. 31 , f. 3, t. 32, t. $1\left(0^{7}\right)$; t. 32, f. 2, 3 (O) (authomedon) (1788). - P. (Eques) a., Gmelin-Linné, Syst. Nat., ed. 13, v. 1. V, p. 2249 (1790). - P. a., Jung, Alphab. Verz. Schmett., v. 1, p. 69 (1791). - J. C. Fabricius, Ent. syst., v. 3. I, p. 87; Index alphab., p. 119 (1793 \& 96). - Fr. Weber, Nomend. Entom., p. 101 (1795). - Turton, Gen. Syst. Nat. Linné, v. 3. II, p. 89 (1806). - ,Automedon", P. a., G. Shaw, Natural. Miscell., v. 20 , t. 839 ; Index (1808). - Oken, Lehrb. Naturg., v. 1, p. 746 (1815). - Morpho a.s Pavonia a., (Latreille \&) J. B. Godart, Enc. Méth., v. 9, p. 447, 807 (1824), - Pavonia a., Lacordaire, Ann. Soc. Ent. France, v. 2, p. 396 (1833). - Verloren, Oat. Ins., Lep. Cramor, v. 1 , p. 29,144 ; v. 2 , p. 202 (1837). Caligo a., (E. Doubleday \&) J. O. Westwood, Gen. Diurn. Lep., v, 2, p. 342 (1851). Chenu \& H. Lucas, Enc. Hist. Nat., Pap. Diurn., p. 168 (1851-53). - Ménétriés, Enum. Corp. Anim. Ac. Petropol., Lep., pars 1; p. 38 (1855). - Herrich-Schäffer, Corr.- 
Bl. Zool.-Min. Ver. Regensb., v. 19, p. 65 (1865). - A. G. Butler, Cat. Diurn. Lep. Fabr., p. 40 (1869). - Eryphanis spec. typ., Boisduval, Consid. Lép. Guatemala, p. 57 (1870). - Caligo a., Eryphanis a., W. F. Kirby, Cat. Diurn. Lop., p. 128 (1871). Pavonia a., Burmeister, Rov, Mag. Zool. ser. 3, v. 1, p. 141 (1873). - W. F. Kirby, Cat. Coll. Diurn. Lep. Hewitson, p. 110 (1879). - Eryphanis a., O. Staudinger (\& Schatz), Exot. Schmett., v. 1, p. 217 (1887).

- Caligo a., L. Glaser, Cat. Etymol. Col. Lep., p. 358 (1887). - Röber: O. Staudinger \& Schatz, Exot. Schmett., v. 2, p. 194 (1889). - O. Michacl, Deutsch. Ent. Zeit. Lep. (Tris), v. 3, p. 149 (1890). - Id., 1. c., v. 7, p. 232 (1895). - Eryphanis a., W. J. Kaye, Ent. Rec. Journ. Var., v. 11, p. 57 (1899). - "automedon", Fruhstorfer: A. Seitz, Großschmett. Ende, v. 5, t. 64 b, c (corr. p. 1140) (1912 \& 24).

a u to meda ena (IÏ̈bn.) Moera a., Jac. Hiibner, Verz. Schmett., p. 51 (1818). - Potamis a., id., Cat. Coll. Lép. Franck, p. 79 [1826].

subsp. W $a \cdot r d i$ Boisd. E. w. Boisduval, Con- Mato Grosso sid. Lép. Guatemala, p. 58 (part.) (1870).

- W. F. Kirby, Cat. Diurn. Lep., App., p. 646 (1871). - H. Stichel, Ins.-Börse, v. 21, p. 197 (sine synon.) (1904). - E. polyxena wardi, Fruhstorfer: A. Seitz, Großschmett. Erde, v. 5, p. 311 (1912).

E. $p$. a $m p h$ imedon, II. Stichel, Gen. Ins., Fasc. 20, p. 32 (part. in cit.) (1904). Id., Tiorreich, Lief. 25, p. 146 (part. in cit.) (1909).

subsp. amphimedon (Teld.) Pavonia a., Santa C. \& R. Felder, Reise Novara, v. 2. II, p. Catharina, 454 (1866). - Caligo automedon var. a, Paraguay, Eryphanis automedon var. a, W. F. Kirby, Argentinien Cat. Diurn. Lep.; p. 129, App., p. 646 (1871). - Eryphanis amphimedon, F. D. Godman \& O. Salvin, Biol. Centr.-Amer., Lep.-Rhop., v. 1, p. 137 (1881). - O. Staudinger (\& Schatz), Exot. Schmett., v. 1, p. 217 (1887). - A. Seitz, Ent. Zeit. Stettin, v. 51, p. 30 (not. biol.) (1890). - E. polyxena a., $\mathrm{H}$. Stichel, Gen. Ins., Fasc. 20, p. 31 (nonnull. cit. except.) (1901). - Id., Tierneich, Lief. 25, p. 146 (ut antea) (1909). - Fruhstorfer: A. Seitz, Grolschmett. Erde, v. 5, p. 311 (1912). - E. a., P. Köhler, Fauna Argent., pars 1 (Zeit. wiss. Ins.-Biol., v. 18 , Beiheft), p. 22 (1923).

Pavonia a utomedon, Burmeister, Rev. Mag. Zool., ser. 3, v. 1, p. 41 (1873). Eryphanis a., Bönninghausen, Verh. Ver. Naturw. Unterh. Hamburg, v. 9, p. 38 (1896).

E. polyxena (non Pap. p. Meerb.), Wey- 
mer (\& Maassen), Lep. Reise Stübel, p. 90 (part. in cit.) (1890). - ? P. Köhler, Fauna Argont., pars 1, (Zeit. wiss. Ins.-Biol., v. 18, Beiheft) p. 22 (1923).

subsp. cheiremon Fruhst, E. p. c., Fruh- Bolivia storfer: A. Seitz, Großschmett. Errde, v. 5, p. 311 (1912).

subsp. tristis Stauding. E. automedon var. Süd-Peru t., O. Staudinger (\& Schatz), Exot. Schmett., v. 1, p. 217 (1887). - E. polyxena t., H. Stichel, Ins.-Börse, v. 21, p. 197 (1904). Id., Gen. Ins., Fasc. 20, p. 32 (1904). - Id., Tierreich, Lief. 25, p. 147 (1909). - Fruhstorfer: A. Seitz, Großschmett. Erde, v. 5, p. 311 (1912).

E. a utomedon (non Pap. a. Cramer), O. Staudinger (\& Schatz), Exot. Schmett., v. 1, t. 76 (1886).

subsp. novicia Stich. E. p. n., H. Stichel, Ecuador Gen. Ins., Fasc. 20, p. 32 (1904). - Id., Ins.-Börse, v. 21, p. 197 (1904). - Id., Tierreich, Lief. 25, p. 147, f. 35 (1909). Fruhstorfer: A. Seitz, Großschmett. Erde, v. 5, p. 311 (1912).

E. a utomed on (uon Pap. a. Cramer), Dognin, Lép. Loja, p. 34 (1891).

subsp. lycomedon (Feld.) Pavonia l., C, Mittel\& R. Felder, Wien. Ent. Monatschr., v. 6, p. Amerika, 423 (1862). - Caligo l., Herrich-Schäffer, Colombia, Corr.-Bl. Zool.-Min. Ver. Regensb., v. 19, p. 65 (1865). - Pavonia l., C. \& R. Felder, Reise Novala, v. 2. II, p. 454, t. 65 , f. 3 (1866). - Caligo l., Eryphanis l., W. F. Kirby, Cat. Diurn. Iop., p. 129; App., p. 646 (1871). - C. automedon var. l., Möschler, Verh. Zool.-IBot. Ges. Wien, v. 26, Abh., p. 322 (in cit.) (1877). - Eryphanis a. forma l., O. Staudinger (\& Schatz), Exot. Schmett., v. 1, p. 217 (1887). - E. l., Therese von Bayern, Berlin. Ent. Zeit., v. 46, p. 265 (sine syn.) (1901). - E. polyxena l., H. Stichel, Gen. Ins., Fasc. 20, p. 32 (cit. part.) (1904). - Id., Ins.-Börse, v. 21, p. 197 (1904). Id., Tiorreich, Lief. 25, p. 147 (cit. part.) (1909). - E. l., Fassl, Soc. Ent., v. 25, p. 39 (ovum) (vido spintharus) (1910). - $E$. polyxena l., Frulistorfer: A. Seitz, Großschmett. Eivle, v. 5, p. 311 (1912). - Eryphanes (!) l., Fassl, Soc. Ent., $\vee .31$, p. 37 (1914). - Id., l. e., v. 35, p. 31 (1918). E. polyxena $l$., Strand, Iep. Niepeltiana, v. 2, p. 12 (specim. parvum Q) (1916). .

$P a v o n$ a a utomedon (non Pap. a. Cramer), F. D. Godman \& O. Salvin, Proc. Zool. Soc. London, p. 11 (1863). H. W. Bates, l. c., p. 249 (1863). - Caligo $a$; A. G. Butler \& Herb. Druoe, 1. c., p. 339 (1874). - Eryphanis a.y F. D. Godman \& O. Salvin, Trans. Ent. Soc. I,ond., p. 122 
(1880). - ? H. G. Dyar, Proc. Un. St. Nat. Mus., v. 47 , p. 144 (1914).

Eryphanis Wardii (part.), Boisduval, Consid. Lép. Guatemala, p. $58(1870)$. $\rightarrow E$. wardi, F. D. Godman \& O. Salvin, Biol. Centr.-Amer., Lep.-Rhop., v. 1, p. 137 (cit. part.), t. 13, f. 1, 2 (1881). - ? E. wardii, Röber: $O$. Staudinger \& Schatz, Exot. Schmett., t. 32 (Morphol.), p. 194 (18s8 $\& 89)$.

forma costaricensis Strand $E$. polyxena Typ.:

lycomedon forma c., Strand, Lep. Niepel- Costa Rica tiana, v. 2 , p. 12, t. 15, f. 7, 8 (1916). Id., Bull. Soc. Zool. Franoe, v. 51, p. 398 (1926).

subsp. s $p$ intharus Fruhst. E. p. s., Fruh- Ost-

storfer, Ent. Rundschau, v. 29, p. 15 (1912). Colombia

- Id.: $\Lambda$. Seitz, Großschmett. Erde, v. 5,

p. 311 (1912). - Eryphanes (!) p. forma s,

Fassl, Fint. Rundschau, v. 33, p. 26 (1916).

E. 8., id., 1. c., v. 35 , p. 31 (1918).

? E. lycomed o n, Fassl, Soc. Ent., v. 25, p.

39 (1910) (vide etiam lycomedon).

\section{Sectio Tricothamnodes}

H. Stichel, Gen. Tns., Fasc. 20, p. 32 (1904). - Id., Tierreich, Lief. 25, p. 148 (1909).

aesacus (Herr.-Schäff.) Caligo $a_{2}$, Herrich-Schäffer, Mittel-

Außercur. Schmett., t. [3] (1850). - Ery- Amerika, phanis a., H. Stichel, Gen. Ins., Fase. 20, p. Colombia, 32 (1904). - Id., Tierreich, Lief. 25, p. West148 (1909). - Fruhstorfer: A. Seitz, Groß- Amazonas schmett. Erde, v. 5, p. 313 (1912).

subsp. a s s c us (Herr.-Schäff.) Caligo a. Mexiko-

(Pavonia a. Kraden MS), Herrich-Schäffer, Nicaragua

ut antea, t. [3], f. 3,4 ; p. $55(1850 \& 58)$

(conf. Dalla Torre, Entom. Nachr. Bl., v. 1, p. 6, 56). - C. a., (E. Doubleday \&) J. O. Westwood, Gen. Dium. Lep., v. 2, p. 342 (1851). - Merrich-Schäffer, Corr.-B1. Zool.Min. Ver. Regensb., v. 19, p. 63 (1865). Eryphane (!) a., Boisduval, Consid. Lép. Guatemala, p. 58 (1870). - Caligo a., Eryphanis a., W. F. Kirby, Cat. Diurn. Lep., p. 129,646 (1871). - E. a., id., Entomologist, v. 11, p. 27 (1878). - Pavonia a., id., Oat. Coll. Dium. Lep. Hewitson, p. 110 (1879). - Eryphanis u., F. D. Godman \& O. Salvin, Biol. Centr.-Amer., Lep.-Rhop., v. 1 , p. 137 (1881). - O. Staudinger (\& Schatz), Exot. Schmett., v. 1, t. 76; p. 217 (1886 \& 87). - Räber, 1. c., v. 2, p. 194 (1889). E. a. a., H. Stichel, Gen. Ins., Fasc., 20, p. 32 (1904). - Id., Tierreich, Lief. 25, p. 150 (1909). - Fruhstorfer: A. Seitz, Großschmett. Erde, v. 5, t. 64 b; p. 313 (1911 \& 12). - E. a., Gibbs, Entomologist, v. 11, p. 27 (1912). 
- subsp. A. G. Butler, Cistula Ent., v. 1, p. 74 (1872). Colombia - A. G. Butler \& Herb. Druce, Proc. Zool. Soc. London, p. 339 (1874). - W. F. Kirby, Cat. Dium. Lep., Suppl., p. 717 (1877). Eryphanis b., F. D. Godman \& O. Salvin, Biol. Centr.-Amer., Lep.-Rhop., v. 1, p. 138, t. 15 , f. 1-3 (1881). - E. aesacus forma $b$., O. Staudinger (\& Schatz), Exot. Schmett., v. 1, p. 217 (1887). - E. a. buboculus, H. Stichel, Gen. Ins., Fasc. 20, p. 32 (1904). Id., Tierreich, Lief. 25, p. 151 (1909). Fruhstorfer: A. Seitz, Großschmett. Erde, v. 5 , p. 313 (1912).

subsp. $j$ uruan $n$ s Fruhst. E. a. juruana, WestFruhstorfer: A. Seitz, Großschmett. Erde, Amazonas v. 5 , p. 813 (1912).

(Rio

Jurua)

Gerhardi (Wcoks) Caligo g., A. G. Weeks, Proc. New Bolivia, Engl. Zool. Club, v. 3, p. 3 (1902). - Id., Ecuador Tlustr. Diurn. Lep., p. 46, t. 12 (1905). Eryphanis g., H. Stichel, Gen. Ins., Fasc. 20, p. 32 (1904). - Caligo oileus g.?, Fruhstorfer, Deutsch. Ent. Zoit. Iris, v. 16 (1903), p. 317 (1904). - Eryphanis g., H. Stichel, Tierreich, Lief. 25, p. 151 (1909). - Fruhstorfer: A. Scitz, Großschmctt. Ende, v. 5, p. 313 (1912). - E. g., E. gehrardi (!), Le Cerf, Enc. Fnt., ser. B. 3, Lep. 2, p. 59, t. 5 , f. $1\left(\sigma^{7}\right)$, f. 2,3 (Q) $(1927)$.

Reevesii (Doubl.) Opsiphanes r., T. Doubleday (\& Bahia Westwood), Gen. Diurn. Lep., t. 57 (1849). bis - Eryphanis r., H. Stichel, Gen. Ins. Fase. Paraguay 20 , p. 153 (1904). - Id., Tierreich, Lief. und 25, p. 153 (1909). - Fruhstorfer: A. Seitz, Argentinien Großschmett. Erde, v. 5, p. 312 (1912). 、

subsp. Reevesii (Doubl.) Opsiphanes r., E. BahiaDoubleday, ut antea, t. 57, f. 3 (Q) (1849); Sta.

Caligo r., J. O. Westwood, l. c., v. 2, p. 342 Catharina, (C. automedon var. Q ?) (1851). - C. r., Minas Chenu \& H. Lacas, Enc. Hist. Nat., Pap. Geraes Diurn., p. 16S, f. $279(1851-53)$. - Herrich-Schäffer, Corr.-Bl. Zool.-Min. Ver. Regensb., v. 19 , p. 65 (1865). - C. r., Eryphanis r., W. F. Kirby, Cat. Diurn. Lep., p. 129; App., p. 646 (1871). - Pavonia rivesii (!), Burmeister, Rev. Mag. Zool., sor. 3, v. 1 , p. $41(1873)$. - P. reevesii, W. F. Kirby, Cat. Coll. Diurn. Lep. Hewitson, p. 111 (1879). - Opsiphanes rivesii, G. W. Müller, Zool. Jahrb. Syst,, v. 1, p. 598 (larva)! (1886). - Eryphanis reevesii, 0 . Standinger (\& Schatz), Exot. Schmett., v. 1, p. 217 (1887). - P E. reveesii (!), Theymer, Ent. Zeit. Stettin, v. 55 (1894), p. 322 [an E. $r$. pusillus?] (1894). - E. reevesii, Bönninghausen, Verh. Ver. Naturw. Unterh. Hamburg, v. 9, p. 38 (1896). - E. r. r., H. 
Stichel, Gen. Ins., Fasc. 20, p. 33 (1904) Caligo r., Silva, Relnt. Contrib. Hist. Nat. Lep. Brasil (Congr. Scient. Lat.-Amer., 1905, v. 3. B), p. 93 ; t. 18 , f. 59 (Caligo automedon, corr.: Errata, p. 181) (1907). - Eryphanis $r$. r. H. Stichel, Tierreich, Lief. 25, p. 154 (1909). - Truhstorfer: A. Seitz, Großschmett. Erde, v. 5, p. 312 , t. 64 b (1912). - E. r., Ledere1, IKandb. prakt. Entom., v. 2, p. 62 (notul. sec. A. Seitz) (1921). $E$. r., Te Cerf, Enc. Hat., ser. B. 2, Lep., v. 2, p. 59 (1927). - Zikán, Ent. Rundschau, v. 45 , p. 11 (1928).

he michroa (Butl.) Caligo h., A. G. Butler, Oistula Ent., v. 1, p 3 (O) (1869). - Id., Lep. Exot., p. 29, t. 11, f. 2 (1870). - C. h., Eryphanis h., W. F. Kirby, Cat. Diurn. Lep., p. 129; App., p. 646 (1871). - E. r., O. Staudinger (\& Schatz), Exot. Schmett., v. 1, p. 217 (1887). - E. reevesii h., Fruhstorfer: A. Seitz, Großsclimett. Erde, v. 5, p. 312 (1912).

E. a utomedon (part.: O), Boisduval, Consid. Lép. Guatemala, p. $58(1870)$. - W. F. Kirby, Cat. Dium. Lep., App., p. 646 (1871). subsp. pus ill $u$ s Stich. E. r. p., II. Stichel, Paraguay, Ins.-Börse, v. 20, p. 359 (1904). - Id., Argentinien, Gen. Ins., Fase. 20, p. 33 (1901). - Id., Rio Grande Tierreich, Lief. 25 , p. 155 (1909). $-E$. gerhardi $p$., Fruhstorfer: $\Lambda$. Seitz, Großschmett. Erde, v. 5, p. 313 (1912). - E. p., Le Cerf, Enc. Ent., ser. B. 3, Lep., r. 2, p. 60 (1927). E. reveesi (!), Weymer, Ent. Zeit. Stettin, v. $55(1894)$, p. $322(1895)$. — ? E. reevesi, P. Köhler, Fauna Argent., pars 1 (Zeit. wiss. Ins.-Biol., v. 18, Beiheft), p. 22 (1923).

zolvizora (Hew.) Pavonia z., Ilewitson, Exot. Butt., Boliviav. 4 (5), t. [6]. Pavonia $2(1876)$. - Ery- Colombia phanis z., II. Stichel, Gen. Ins., Fasc. 20, p. 33 (1904). - Id., Tierreich, Lief. 25, p. 155 (1909). - Fruhstorfer: A. Seitz, Großschmett. Erde, v. 6, p. 312 (1912).

subsp. zolvizora (Hew.) Pavonia z., He- Ecuador, witson, nt antea, t. [6], Pavonia 2; Text Peru, (1876 \& 77). - Caligo z., W. F. Kirby, Cat. Bolivia Diurn. Lep.. Suppl., p. 817 (1877). - Pavonia z., id., Cat. Coll. Diurn. Lep. Hewitson, p. 110 (1879). - Caligo z., Weymer (\& Maassen), Lep. Reise Stübel, p. 62 (1890). - Eryphanis z. z., H. Stichel, Gen. Ins., Fasc. 20, p. 33 (1904). - Id., Tierreich, Lief. 25, p. 157 (1909). - Fruhstorfer: A. Seitz, Großschmett. Erde, v. 5, p. 312 (1912).

E. opimu 8, O. Staudinger (\& Schatz), Exot. Schmett., v. 1, p. 217 (part.) (1887). Dognin, Lép. Loja, p. 72 (1894).

subsp. o p $i m$ us Staud. E. o., O. Staudinger Colombia (\& Scliatz), Exot. Schmett., v. 1, p. 217 (distr. part.) (1887). - Therese von Bayern, 
Berlin. Ent. Zeit., v. 47 (1901), p. 265 (1902). - E. zolvizora o., H. Stichel, Gen. Ins., Fasc. 20, p. 33 (1901). - Id., Tierreich, Lief. 25 , p. 157 , f. $36(1909)$. - $E$. o., Fassl, Soc. Ent., v. 24, p. 115 (not. biol.) (1909). - E. z. o., Fruhstorfer: A. Seitz, Großschmett. Erde, v. 5, p. 312 (1912). Eryphanes (!) o., Fassl, Ent. Rundschau, v. 33, p. 26 (1916). - Id., Soc. Ent., v. 35, p. 5 31 (1918).

\section{Genus Caligopsis Seyd.}

C. (Subgenus), O. Seydel, Neue Beitr. syst. - Ins.kunde, v. 3, p. 30 (1924).

Pavonia (part.), Hewitson, Exot. Butt., v. 5 (4), t. [5] Pavonia; Text (1876 \& 77). - W. F. Kirby, Cat. Diurn. Lep. Hewitson, p. 110 (1879).

Caligo (part.), W. F. Kirby, Cat. Diurn. Iep., Suppl., p. 847 (1877).

Eryphanis (part.), H. Stichel, Gen. Ins., Fasc. 20, p. 30, 33 (1904). - Id., Tierreich, Lief. 25, p. 141, 158 (1909). - Fruhstorfer: A. Seitz, Großschmett., v. 5, p. 310, 312 (1912). - ? Fassl, Ent. Zeit., v. 36, p. 25 (1922).

seleucida (Hew.) Pavonia s., Hewitson, Exot. Butt., Bolivia v. 4 (5), t. [5] Pavonia; Text (1876 \& 77). - Caligo s., W. F. Kirby, Cat. Diuın. Lep., Suppl., p. 847 (1877). - Pavonia s., id., Cat. Coll. Diurn. Lep. Hewitson, p. 110 (1879). - Eryphanis s., II. Stichel, Gen. Ins., Fasc. 20, p. 33 (1909). - Id., Tierreich, Lief, 25, p. 158 (1909). - Fruhstorfer: A. Seitz, Großschmott. Ende, v. 5, p. 312 (1912). - E. (Caligopsis) s., O. Seydel, Neue Beitr. syst. Ins.-kunde, v. 3 , p. 30 (1924)

Dondoni (Fassl) E. d., Fassl, Ent. Zeit., v. 36, p. Amazonas 2

25 (1922) (spec. inc. sed., ? subsp. pracced.)

\section{Genus Caligo Jac, Hübn.}

Jac. Hübner, Verz. Schmett., p. 51 [1818]. - (E. Doubleday \&) J. O. Westwood, Gen. Diurn. Lep., v. 2, p. 340 (part.) (18.51). Chenu \& H. Lucas, Enc. Hist. Nat., Pap. Diurn., p. 167 (part.) (1851-53). - C. (or Pavonia), Wallace, Trans. Ent. Soc. London, n. ser., v. 2, p. 261 (not. biol.) (1854). - Mrénétriés, İnum. Corp. Anim. Ac. Petropol., Lep., pars 1, p. 38 (1855), - J. O. Westwood, Trans. Ent. Soc. Iondon, n. ser., v. 4, p. 165 (1856). - Fauvel, Bull. Soc. Linn. Normand,, v. 6 (1860-61), p. 139 (1862). - Carus-Gerstäcker, Handb. Zool., v. 2, p. 231 (1863). Herrich-Schäffer, Corr.-Bl. Zool.-Min. Ver. Regensb., v. 18, p. 55; v. 19, p. 65 (1864 \& 65). - Prittwitz, Ent. Zeit. Stettin, v. 26, p. 309 (1865). - G. Koch, Indo-Austral. Lep.-Fauna, ed. 1, 2, p. 11 f (1865 \& 73). - A. G. Butler, Cat. Diurn. Lep. Fabr., p. 39 (part.) 
(1869). - Deyrolle, Rev. Mag. Zool., ser. 3, v. 21, p. 18 (1872). - W. F. Kirby, Cat. Diurn. Lep., p. 127 (part.), p. 646; Suppl., p. 717 (part.) (1871 \& 77). - A. G. Butler \& Herb. Druce, Proc. Zool. Soc. London, p. 338 (part.) (1874). - S. H. Scudder, Hist. Sketch: Proc. Amer. Ac. Sci, v. 10, p. 129 (1875). - A. G. Butler, Proc. Zool. Soc. London, p. 217 (1876). - Wallace, Geogr. Distrib. Anim., v. 2, p. 472 (1876), - Möschler, Verh. Zool.-Bot. Ges. Wien, v. 26, Abh., p. 321 (part.) (1877). - Fritz Müller, Jena. Zeit. Naturw., v. 11, p. 102 (Morphol.) (1877). - A. G. Butler, Trans. Ent. Sac. London, p. 113 (1877). - Fritz Müller, Ent. Zeit. Stettin, v. 39, p. 296 (1878). - Id., Traus. Ent. Soc. London, p. 214 (odor) (1878). - Burmeister, Descr. Phys. Argent., v. 5, pars 1, p. 199 (1878). - W. F. Kirby, Entomologist, v. 11, p. 27 (1878). - J. H.: G. Jäger, Handwörterb. Zool., v. 1, p. 501 (1880). - F. D. Godman \& O. Salvin, Biol. Centr.-Amer., Lep.-Phop., v. 1, p. 130 (1881). - O. Orüger (soc. Hewitson), Berlin. Ent. Zeit., v. 25, p. 111 (1881). - E. Gerhard, ibid., v. 27, p. 179 (distrib. geogr.) (1883). - G. W. Müllor, Zool. Jahrb. Syst., v. 1, p. 605 (1886). - O. Staudinger (\& Schatz), Exot. Schmett., v. 1, p. 215 (1887). - L. Glaser, Cat. Etymol. Col. Lep., p. 278 etc. (1887). - Knaver, Handwörterb. Zool., p. 124 (1887). - Röber: O. Staudinger \& Schatz, Exot. Schmett., v. 2, t. 32 (Morphol.); p. 191, 193 (1888 \& 89). - Iticholmann, Tagebl. Deutsch. Naturf. Versamml., 61, p. 68 (1889). - Hahnel, Deutsch. Ent. Zeit. Lop. (Iris), p. 14 s etc. sec. indicem p. 324 (1890) - A. Seitz, Ent. Zeit. Stettin, v. 51, p. 30 (1890). - W. F. Kirby, Handb. Lep., v. 1, p. 200 (1894). - O. Michael, Deutsch. Ent. Zeit., Lep. (Iris), v. 7 (1894), p. 202 (not. biol.) (1895). - E. Reuter, Acta Soc. Sci. Fenn., v. 22, p. 113, 553 (1896). - W. F. Kirby, Samml. Exot. Schmott., Hübner \& Geger, Neue Ausg., v. 3, p. 52 (1901). - Caligo(s), W. J. Kaye, Trans City London Ent. Nat. Hist. Soc. (1902), p. 54 (1903). - Caligo, $\mathrm{H}$. Stichel, Gen. Ins., Fasc. 20, p. 33, t. 2, f. 4 (Morphol.) (1904). - Fruhstorfer, Deutsch. Ent. Zeit., Iris, v. 16 (1903), p. 313 (1904). - Silva, Relat. Contrib. Hist. Nat. Lep. Brasil (Congr. Scient. Lat.-Amer., 1905, v. 3. B), p. 90 (part.) (1907). - H. Stichel, Tierreich, Lief. 25, p. 158, f. 37 (Morphol.) (1909). A. Pagenstecher, Geogr. Verbr. Schmett., p. 413 (1909). - C., Caligulo, Fruhstorfer: A. Seitz, Großschmett. Erde, v. 5, p. 314, p. 325 (corr. p. 1134) (1912). - Caligo, W. Rothschild, Novit. Zool., v. 23, p. 312 (1916). - W. J. Kaye, Proc. Zool. Soc. London, p. XIX (1917). - Schierbeck, Onderzoek. Zool. Labor. Groningen, Nr. 6, p. 11 (Morphol. larv.) (1917). - Strand, Soc. Entom., v. 33, p. 19 (1918). - Calligo(8), Halliman, Journ. N. York Ent. Soc., v. 2S, p. 238 (1920). - Caligo, G. Lederer, Handb. prakt. Entom., v. 2, p. 61 (Phyto- et Zoomimose sec. A. Seitz) (1921). - Campos, Rev. Col. Nacion. Viceute Rocafuerte, Nr. 4, p. 30 (1921). Giacomelli, Rev. Ohil. Hist. Nat., v. 27, p. 16 (Mimikry) (1924). Schwanwitsch, Proc. Zool. Soc. London, p. 521 (Morphol.) (1924). - Handlirsch: Chr. Schröder, Handb. Entom., v. 3, p. 939 (1925). - M. Horing, Biol. Schmett., p. 322 (Phyto-, Zoomimose; Kaenophylaxis sec. Ieikertinger). - Le Cerf, Enc. Ent., ser. B. 3, Irep., v. 1, p. 141 (Anatom.) (1926). - Prochnow: Chr. Schröder, Handb. Entom., v. 2, p. 562 (color) (1927). - Holdhaus, ibid., v. 2, p. 731, 737 (distrib. geogr.) (1927). - A. Seitz, Ent. Rundschau, v. 45, p. 31 (1828).

$E$ que s [Subgenus] (part.), Linné, Syst. Nat., ed. 10, p. 458,464 (1758). - Id., 1. c., ed. 12 , v. 1. 2 , p. 744,753 (1767). - Achivus [Subgenus] (part.), P. L. S. Müller, 
Naturs. Linné, v. 5. 1, p. 579 (1774). - Eques (Achivus) (part.), [D. H. Schneider], Nomencl. Entom., p. 33 (1785). - L. [Subgen.] (part.), Linné-Houttyn, Natuur]. Hist., v. 1. XI, p. 216 (1766-69). - Gmelin-Linné, Syst. Nat., od. 13, v. 1. V, p. 2248 (1790). - E. (Achivus) (part.)', Esper, Ausl. Schmett., p. 179, 182 (1801).

Heros [Subgenus], Illiger, Mag. Ins.-kunde, v. 1, p. 199 (1801).

"Potamis conspicua", Jac. Hübner, Samml. Exot. Schmett., v., t. [77] (1806-19). - Potamis (part.), id., Cat. Lép. Franck, p. 79 (1826). - ,P. Conspicul", W. F. Kirby, Samml. Exot. Schmett. Hübner \& Geyer, Neue Ausg., v. 1, t. 77 (1894).

„S a ty r e" (part.), Iatreille, Consid. Gén., p. 440 (1810).

A erodes (Dilman MS), Billberg, Enum. Ins., p. 79 (1820). - S. H. Scudder, Hist. Sketch: Proc. Am. Ac. Sci., v. 10, p. 104 (nom. pracocc.) (1875).

Ha madrya s, Mikan, Delect. Flor. Faun. Brusil., t. 12, expl. (1821).

Morpho (part.), Latreille (\& Godart), Enc. Méth., v. 9, p. 435 ; J. B. Godart, l. e., p. 448 etc. (1821). - H. Lucas, Hist. Nat. Lép. Exot., p. 138 (1835). - Gistel, Naturg. Thierreich, p. 145 (1848). - Kollar, Denkschr. Ak. Wien, v. 1, p. $354(1849)$. - Kollar, Abh. Naturf. Ges. IIalle, v. 2, Sitıgsber., p. 62 (1854).

Pavonia (part.), (Tatreille \&) J. B. Gadart, Enc. Méth., v. 9, p. 807 (1824). - "Paconie", Latreille, Fam. Nat. Règne Anim., p. 46S; p. 469 (soc. Godart) (1825). Paronia (part.), A. Berthold: Latreille, Nat. Fam., p. 473; p. 474 (sec. Godart) (1827). - P., Burmoister, Ins. Syst. Nat., p. 27 (1829). - P. (part.), Griffith-Cuvier, Anim. Kingdom, v. 15, p. 590 (1832). - Lacordaire, Ann. Soc. Ent. France, v. 2 , p. 396 (1833). - Boisduval (sec. Latreille), Spec. Gén. Lép., v. 1, p. 118. (1836). - Id.: Cuvier, Règne Anim., ed. 3, p. 231 (1836). - P., Jam. Duucan, Foreign Butterfl. (Jandine, Nat. Libr., v. 36, Eutom. 5) p. 178 (1837). - Verloren, Cat. Ins. Lep. Cramer, v. 2, p. 202 (1837). - Burmeister, Handb. Naturg., p. 23 (1837). - F. S. Toigt: Cuvier, Thierreich, v. 5, p. 535 (part.) (1839). - E. Doubleday, List Lep. Brit. Mus., v. 1, p. 117 (1844). - L[ucas]: Orbigny, Dict. Hist. Nat., v. 9, p. 515 (part.) (1847). - Erichson: Schomburgk, Guiana, v. 3, p. 599 (1848). - E. Doubleday (\& Westwood), Gen. Diurn. Lep., t. 56 (1849). - Herrich-Schäffer, Samml. Außereur. Schmett., Lief. 1, Index (1850). - P. (Subgenus), Hoeven, Handb. Zool., ed. 2, v. 1, p. 398 (1850). - (Caligo or) $P$. Wallace, Trans. Ent. Soc. London, n. ser., v. 2, p. 261 (not. biol.) (1854). - P., C. \& R. Felder, Wien. Ent. Monatschr., v. 6, p. 123 (1862). - Piffard, Ent. Monthly Mag., v. 1, p. 80 (1864). - H. W. Batesj Journ. Entom., v. 2, p. 176 (186t). - C. \& R. Felder, Reiso Novara, v. 2. II, p. 454 (part.) (1866). - Boisduval, Consid. Lép. Guatemala, p. 55 (part.) (1870). - P. (Caligo + Opsiphanes), Burmeister, Rov. Mag. Zool., ser. 3, v. 1, p. 19, 31, 36 (1873). - P., Capronnier, Ann. Soc. Ent. Belg., v. 17 , p. 28 (1874). - S. H. Scudder, Hist. Sketch: Proc. Am. Ac. Sci., v. 10, p. 242 (nom. praeoce.) (1875). - W. F. Kirby, Cat. Coll. Diurn. Lep. Hewitson, p. 110 (1879). - Capronnier, Ann. Soc. Ent. Belgique, v. 25, p. 
101 (1881). - G. C. Champion, Ent. Monthly Mag., v. 19, p. 227 (1883). - L. Glaser, Cat. Etymol. Col. Lop., p. 306 (1887).

A e r o n a u ta (part.), Berge, Schmetterlingsb., p. 107 (1842). Eryphanis (part.), Ménétrićs, 'Enum. Corp. Anim. Ac. Petropol., Lep., pars 1, p. 38 (1855).

\section{Sectio Graphiophori}

H. Stichel, Gen. Ins., Fasc. 20, p. 35 (1904). - Id., Tierreich, Lief. 25, p. 162 (1909), - Fruhstorfer: A. Seitz, Großschmett. Erde, v. 5, p. 317 (1912).

\section{a. Cohors Teucriformes}

H. Stichel, ut antea (1904 \& 09).

teucer (I.) Papilio (Eques) t., Linné, Syst. Nat., Nördliches ed. 10 , p. 464 (1758). - Caligo t., H. Sti- und chel, Gen. Ins., Fasc. 20, p. 35 (1904). - westliches Id., Tierreich, Lief.. 25, p. 162 (1909). - Süd-

Fruhstorfer: A. Seitz, Großschmett. Erde, Amerika v. 5, p. 317 (1912). - W. J. Kaye, Proc. bis Ent. Soc. London, p. XIX, XX (part.) (1917). Paraguay

subsp. te ucer (L.) - Merian, Metam. Ins. Guayana, Surin., t. 23 (1705) (conf. Burmeister, Abh. Venezuela, Naturf. Ges. Ilalle, v. 2, 1854, Sitzgsber., p. Trinidad, 62: Morpho teucer). - ? Petiver, Gazoph. Nord-

Nat. Art., t. 28 , f. 1 (1709 \& 67) (sec. Brasilien, Aurivillius, $1882,=C$. ilioneus, sed errans Norlostputo, conf. W. F. Kirby, 1870). - Gronov Peru (Gronovius), Zoophyl., Fasc. 2, p. 187, Nr. 721 (1764). - Merian-Buchoz, Hist. Ins. Surin., ed. 3, v. 1, p. 23; v. 2, p. 49: „Teucer" (1771). - Papilio (Eques) teucer, Linné, Syst. Nat., ed. 10, p. $46 \pm$ (1758). - P. t., id. (Rydbeck), Amoen. Acad., v. 5, p. 252 (1758). - Linné, Mus. Ludov. Ulr., p. 212 (1746). - P. (Eques) t., id., Syst. Nat., ad. 12, v. 1. II, p. 753 (1767). - IinnéHouttyn, Natuurl. Hist., v. 1. XI, p. 216 (1767). - $P$. (Achivus) teuces (err. typ.), P. L. S. Müller, Naturs. Linné, v. 5. I, p. 579 (1774). - P. teucer, J. O. IRabricius, Syst. Ent., p. 458 (1775). - (Gmelin \&) Christman, Onomat. Med., v. 6, p. 154 (1775). - "Teucer", Papilio t., P. Cramer, Pap. Exot., v. 1, p. 80, t. 51, f. A, B; p. 154 $(1775$ \& 76). - J. H. Sulzer, Abgek. Gesch. Ins., v. 1 , p. 142 ; v. 2 , t. 14 , f. 1 (1776). "Teucer", Meuschen \& Boddaert, Museum Gronovian., p. 70, Nr. 678 (1778). $-P$. t., Goeze, Ent. Beytr., v. 3. I, p. 67 (1779). - J. C. Fabricius, Spec. Ins., v. 2, p. 25 (1781). - C. Stoll, Essai Syst. Lep. (P. Cramer, Pap. Exot., v. 4, App.), p. 8, Nr. 19 (1782). - P. (Eques Achivus) t., [D. H. Schneider ], Nomencl. Entom., p. 33 (1785). - J. C. Fabricius, Mant. Ins., v. 2, p. 13 (1787). - (Jablonsky \&) Herbst, Na- 
turs. Ins., Schmett., v. 3, p. 79, t. 31 , f. 1 , 2 (1788). - J. Roemer, Gen. Ins. Linné Fabr., p. 68, t. 14, f. 1 (1789). - $P$. (Eques) t., Gmelin-Linné, Syst. Nat., ed. 13 , v. 1. V, p. 2248 (cit. part.) (1790). $P$. t., Jung, Alphab. Verz. Schmett., v. 2, p. 232 (1792). - A. G. Olivier, Act. Soc. Hist. Nat. Paris, v. 1, p. 121 (1792). - J. C. Fabricius, Ent. syst., v. 3. I, p. 87; Index alphab., p. 128 (1793 \& 96). - Fr. Weber, Nomencl. Entom, p. 101 (1795). - P. (Eques Achivus) t., Esper, Ausl. Schmett., p. 179, t. 44, f, 1 (1796). - P. tencer (err. typ.), Tableau Encycl. Méth, v. 18, t. 24, f. 3, 3 bis, f. 4 A, $B$ (larva, chrysal., fig. dubiae) (1797). - P. teucer, Latreille, Ilist. Crust. Ins., v. 3, p. 392 (1802). - Thunberg, Mus. Nat. Ac. Upsal. Dissert., pars 23 , p. 9 (1804) (sec. Aurivillius, 1882). - Turton, Gen. Syst. Nat. Iinné, v. 3. II, p. 89 (1806). - Gravenhorst, Vergl. Übers. Zool. Syst., p. 313 (1807). - Latreille, Consid. Gén., p. 440 (1810). - Oken, Iehrb. Naturg., v. 1, p. 746 (1815). - Latroille, Cuvier, Règne Anim., v. 3, p. 545 (1817). - Caligo teucra (!), Jac. Hübner, Verz. Schmett., p. 51 (1818). - Morpho teucer, Pavonia t., (Latreillo \&) J. B. Godart, Enc. Méth., v. 9 , p. $448 ; 807$ (1824). — ,teucer", GriffitlCuvier, Anim. Kingdom, v. 14, p. 95 (1832). - Pavonia t., Verloren, Cat. Ins. Lep. Cramer, v. 1, p. 32; v. 2, p. 202 (1S37). Erichson: Sehomburgk, Guiana, v. 3, p. 599 (1848). - ? Morpho t., Kollar, Denkschr. Ak. Wiss. Wien, v. 1, p. 358 (1849). (E. Doubleday \&) J. O. Westwood, Gen. Diurn. Lep., จ. 2, p. 342 (1851), - Caligo $t$., Chenu \& H. Lucas, Enc. Hist. Nat., Pap. diurn., p. 168 (1851-53). - Norpho $t_{\text {., }}$ Burmeister, Abh. Naturf. Ges. Halle, v. 2, Sitzgsber., p. 58, 62 (1854). - Caligo t., Ménétriés, Enum. Corp. Anim. Ac. Petropol., Lep., pars 1, p. 38 (1855). - J. O. Westwood, Trans. Ent. Soc. London, n. ser., v. 4, p. 165 (1856). - Fauvel, Bull. Soc. Linn. Normand., v. 6 (1860-61), p. 139 (1862). - A. G. Butler, Cat. Diurn. Lep. Fubr., p. 39 (1869). - Papilio t. (Caligo) W. F. Kirby, Trans. Ent. Soe. London, p. 137 (typ. Linn. identif.) (1870). - C. t., id., Cat. Diurn. Isep., p. 128 (1871). Möschler, Verh. Zool.-Bot. Ges. Wien, v. 26, Abh., p. 321 (1877). - Pavonia t., W. F. Kirby, Cat. Coll. Diurn. Lep. Hewitson, p. 110 (1879). - Caligo t., J. H.: G. Jäger, Handwörterb. Zool., v. 1, p. 501 (1880). Chr. Aurivillius, Svenska Ak. Mandl., n. ser., v. 19, Nr. 5, p. 32 (nonnul. cit. except.) (1882). - O. Staudinger (\& Schatz), 
Exot. Schmett., v. 1, p. 216 (part.) (non $t$. 73: = C. illioneus) (1887). - C. t., Pavonia t., L. Glaser, Cat. Etym. Col. Lep., p. 278, 384, 306 (1837). - C. t., Röber: O. Staudinger \& Schatz, Exot. Schmett., v. 2, p. 194 (1889). - E. Reuter, Acta Soc. Sci. Fenn., v. 22 , p. 113 , t. 4 , f. 42 (palpus) (1896). c. t. t., Fruhstorfer, Deutsch. Ent. Zeit. Iris, v. $16(1903)$, p. 319 (1904). - H. Stichel, Gen. Ins., Fasc. 20, p. 35 (cit. part.) (1904). - Id., Tierreich, Lief. 25, p. 165 (forma principalis) (cit. nonnull. except.) (1909). - Fruhstorfer: A. Seitz, Großschmett. Erde, v. 5, p. 317 (1912). - W. F. Kaye, Proc. Ent. Soc. London, p. XXI (1917).

Papilio idomaneus (non Linné), (Jablonsky \&) Herbst, Naturs. Ins., Schmett., v. 3, p. 70 (part. in cit.) (1788).

subsp. o bidona Fruhst. $C$. $t$. obidonus, Amazonas Fruhstorfer, Soc. Ent., v. 18, p. 145 (1904). (Obidos)

- Id., Deutsch. Ent. Zeit. Iris, (1903), v. 16 , p. 314, 319 (1904). - H. Stichel, Gen. Ins., Fasc. 20 , p. 35 (1904). - C. teucer $t$. forma obidona, id., Tierneich, Lief. 25, p. 166 (1909). - C. t. obidonus, Fruhstorfer: A. Seitz, Großschmett. Ende, v. 5, p. 318 (1912). - W. J. Kaye, Proc. Ent. Soc. London, p. XXI (1917). (Subsp. dub., ? forma subsp. typ.).

subsp. joasa Joicey \& Kaye $C, t . j$. Joicey Amazonas \& W. J. Kaye, Ent. Rec. Journ. Var., v. (Rio Joas) 29, p. 180 (1917). - W. J. Kaye, Proc. Ent. Soc. London, p. XXI (1917). (Subsp. dub., ? forma subsp. typ.).

subsp. $n u b i l a$ Fruhst. $C$. $t$. nubilus, Fruhstorfer, Ent. Zeit. Stettin, v. 6S, p. 129, 130 (1907). - C. t. zuzanna (!) forma nubila, II. Stichel, Berlin. Ent. Zeit., v. 52, p. 162 (1908). - C. t. suzanna forma n., id., Tierreich, Lief. 25, p. 169 (1909). - C. t. nubilus, Fruhstorfer: A. Seitz, Grolschmett. Erde, v. 5, p. 318, t. 63 c (1912). (Subsp. dub.,? forma subsp. typ.).

subsp. in sula $n$ a Stich. C. t. insulsmus, H. Trinidad,

Stichel, Ins.-Börse, v. 21, p. 21 (1904). - ? Venezuela

Id., Gen. Ins., Fasc. 20, p. 35 (1904). - Id., Tierreich, Lief. 25, p. 167 (cit. part.) (1909). - Fruhstorfer: A. Seitz, Großschmett. Erde, v. 5, p. 317 (1912). - W. J. Kaye, Trans. Ent. Soc. Lond., p. 548 (sine synon.) (1914). - W. Rothschild, Nov. Zool., v. 23, p. 312 (1916).

- subsp. 8 zanna (Deyr.) Pavonia s., E. Colombia Deyrolle, Rev. Mag. Zool., ser. 3, r. 23, p. 275 , t. 24, 26 (1872). - Caligo s., W. F. Kirby, Oat. Diurn. Lep., Suppl., p. 717 (1877). - O. Staudinger (\& Schatz), Exot. Schmett., v. 1, p. 217 (1887). - C. teucer s., H. Stichel, Gen. Ins., Fasc. 20, p. 36

Britisch-

Guayana 
(1904). - Fruhstorfer, Ent. Zeit. Stettin, v. 68, p. 129 (1907). - C. t. zuzanna (!), H. Stichel, Berlin. Ent. Zeit., v. 52, p. 161 (1908). - C. t. suzanna, id., Tierreich, Lief. 25 , p. 168 , f. 38 (forma principalis): (cit. corrig.) (1909). - W. J. Kaye, Proc. Ent. Soc. London, p. XXI (1917).

subsp. phoroncus Fruhst. C. t. phorancus, Ost-

Fruhstorfer, Ent. Zeit. Stettin, v. 68, p. 129 Colombia (1907). - Id.: A. Seitz, Großschmett. Erde, (Rio Waupes) v. 5, p. 318 (1912). (Subsp. dub., ? forma praceod.).

C. t. $s u z$ an $n a$, H. Stichel, Tierreich, Lief. 25 , p. 168 (in cit.) (1909).

subsp. c cu adora Joicey \& Kaye C. t. e., Ecuador Joicey \& W. J. Kaye, Ent. Rec. Journ. Var., v. 29, p. 181 (1917). - W. J. Kaye, Proc. Ent. Soc. London, p. XXI (1917).

C. teucer (non Pap. t. L.), Dognin, Lép. Loja, p. 34 (1891).

? C. telamonius (non Pavonia t. Felder), Campos, Rev. Col. Nacion. Vivente Rocafuerte, Nr. 4, p. 30 (1921).

subsp. s e mi ca er u $l$ c a Joicey \& Kaye $C$. $t$. Südosts., Joicey \& W. J. Kaye, Ent. Rec. Journ. Peru Var., v. 29, p. 181 (1917). - W. J. Kaye, (La Proc. Ent. Soc. London, p. XXI (1917). Merced)

subsp. phor ky $\&$ Fruhst. C. t. p., Fruhstor- Bolivia fer: A. Seitz, Großschmett. Erde, v. 5, p. 317 (1912).

subsp. ja petu \& Stich. C. t. j., H. Stichel, Paraguay, Ins.-Börse, v. 20 , p. 389 (1903). - C. t. Mato Grosso, iapetus, id., Gen. Ins., Fase. 20, p. 35, t. 5, ? Südf. 1 (1904). - C. t. japetus, id., Tierreich, Brasilien Lief. 25, p. 166 (1909). - Fruhstorfer: A. Seitz, Großschmett. Erde, v. 5, p. 318 (1912). - Talbot, Bull. Hill Mus., v. 2, p. 199 (1928).

illioneus (Cram.) Papilio i., P. Cramer, Pap. Exot., Südv. 1 , p. 153 (1776). - Caligo i., H. Stichel, Amerika Gen. Ins., Fasc. 20, p. 36 (1904). - Id., bis Gren. Ins., Fasc. 25, p. 169 (1909). - Fruh- Paraguay, storfer: A. Seitz, Großschmett. Erde, v. 5, p. 318 (1912).

Panama,

Trinidad,

?Jamaica

subsp. illioneus (Cram.) - Petiver, Ga- Guayana, zophyl. Nat. Art., p. 43 , t. 28 , f. 1 ( 1704 Amazonas, \& 09 ) (vide $\Lambda$ urivillius, Svensk. Vet. Akad. Mato Grosso, Handl., v. 19 , Nr. 5, p. 33, notz 3; sec. W. ParáF. Kirby, Trans. Ent. Soc. London, 1870, Parana; p. 137: tcucer). - Sloane, Nat. Hist. Ja- ?Jamaica maica, v. 1. II, p. 29, Nr. 4 (1707). - „Illio- (fid. neus" ${ }^{i t}$, Papilio i., P. Cramer, Pap. Exot., v. Sloane) 1 , p. 81 , t. 52 , f. A () ; p. $153(1775$ \& 76). - P. (Eques Achivus) i., Groeze, Ent. Beytr., v. 3. I, p. 81 (1779). - P. i., C. Stoll, Essai Syst. Lep. (P. Cramer, Pap. 
Exot., v. 4, App.), p. 8, Nr. 7 (1782). - P. (Eques Achivus) i. P, [D. H.' Schneider], Nomencl. Entom., p. 33 (1785). $-P$. ilioneus, (Jablonsky \&) Herbst, Naturs. Ins., Schmett., v. 3, p. 75, t. 30, f. 1 (P) (non f. 2: $=C$. idomeneus) (1788). $-P$. (Eques) idomencus, 3. P. ilioncus, Gmelin-Linné, Syst. Nat., od. 13, v. 1. $\nabla$, p. $2248(1790)$. $-P$. ilioneus, Jung, Alphab. Verz. Schmett., v. 1, p. 205 (1791). - P. idomencus, B. $P$. illioneus, Fr. Weber, Nomencl. Entom., p. 101 (1795). - Caligo illionea, Jac. Hübner, Verz. Sclinett., p. 51 (part.) (1818). Morpho ilioneus, Pavonia i., (Latreille \&) J. B. Godart, Enc. Méth., v. 9, p. 448; 807 (1824). - P. i., Verloren, Cat. Ins. Lep. Cramer, v. 1, p. 33; v. 2, p. 202 (1837). E. Doubleday, List Lep. Brit. Mus., v. 1, p. 108 (1844). - Erichson: Schomburgk, Guiana, v. 3, p. 599 (1848). - Caligo i., (E. Doubleday \&) J. O. Westwood, Gen. Diurn. Lep., v. 2, p. 342 (1851). - Ménétriés, Enum. Corp. Anim. Ac. Petropol., Lep., pars 1, p. 38 (1855). - Pavonia i., Piffard, Ent. Monthly Mag., v. 1, p. 80 (1864). Caligo i., Herrich-Schäffer, Corr.-Bl. Zool.Min. Ver. Regensb., v. 19, p. 65 (1865). W. F. Kirby, Cat. Diurn. Lep., p. 128 (cit. part.) (1871). - Pavonia i., Burmeistor, Rev. Mag. Zool., ser. 3, v. 1, p. 39, t. 5 (Metamorph.) (1873). - Caligo i., Möschler, Verh. Zool.-Bot. Ges. Wien, v. 26, Abh., p. 321 (1877). - C. Crüger, Verh. Ver. Naturw. Unterh. Hamburg, v. 4 (1877), p. 193 (1879). - F. D. Godman \& O. Salvin, Biol. Centr.-Amer., Lep.-Rhop., v. 1, p. 133 (part.) (1881). - C. i., Pavonia i., L. Glaser, Cat. Etymol. Col. INep., p. 278, 306, 371 (1887). - C. i., O. Staudinger (\& Schatz), Exot. Schmett., v. 1, p. 216 ; t. 73 (falso teucer). (1887 \& 86). - Röber, l. c., v. 2, p. 193 (1889). - W. F. Kirby, Handb. Lep., v. 1, p. 201 , t. 38, f. 2 (fig. mal.) (1894). - Bönninghausen, Verh. Ver. Naturw. Unterh. Hamburg, v. 9 (1894-95), p. 37 (1896). C. illioneus i., H. Stichel, Gcn. Ins., Fasc. 20, p. 36 (cit. part.) (1904). - C. i. i., Fruhstorfer, Deutsch. Ent. Zeit. Iris, v. 16, (1903), p. 314, 320 (1904). - H. Stichel, Tierreich, Lief. 25 , p. 172 (cit. part. et corrigend.) (1909). - C. illioneus $i$., Fruhstorfer: A. Seit $\%$ Großschmett. Ende, v. 5, t. $64 \mathrm{c}$ (,ilioneus ${ }^{\text {ut }}$ ) p. 319 (1911 \& 12). C. illioneus $i$., Cleare, Trans. Ent. Soc. London, v. 74 , p. 361 (cit. part.), t. $81-83$ (Biol.) (1926). - C. ilioneus i., Talbot, Bull. Hill Mus., v. 2, p. 199 (1928).

$P$ apilio idomeneus (non Linné) (part. in eit.), J. O. Fabricius, Spec. Ins., v. 2, 
p. 26 (1781). - Id., Syst. Ent., v. 3. I, p. 88 (1793). - (Jablonsky \&) Herbst, Naturs. Ins., Schmett., v. 3, p. 70 (1788).

Papilio (Eques) teu cer, Gmelin-Linné, Syst. Nat., ed. 13, v. 1. V, p. 2248 (part. in cit.) (1790). - Caligo t., Jam. Duncan, Foreign Butterfl. (Jardine, Nat. Libr., v. 36, Ent. 5), p. 179, t. 22, f. 2 (fig. mal) (1837). - O. Staudinger (\& Schatz), Exot. Schmett., v. 1, t. 73 (1886). - ? Loquai, Int. Ent. Zeit., v. 22, p. 156 (1928).

Aeronauta curilochus (non Papilio e. Cram.), Berge, Schmetterlingsb., p. 107, t. 35 , f. 1 (ㅇ) (fig. mal.) (1842).

Ca ligo oberon (part.), A. G. Butler, Trans. Ent. Soc. London, p. 488 (1870) - C. o., id., l. c., p. 113 (1877). - E. M. Sharpe, Proc. Zool. Soc. London, p. 566 (1890). Brunner-Wattenwyl, Farbenpr. Ins., p. 6 , $t$. 4, f. 52 (1897).

- subsp. polyxenus Stich. C. i. p. (Pavonia Venezuela p. Maassen MS), H. Stichel, Ins.-Börse, v. 20 , p. 389 (1903). - Id., Gen. Ins., Fasc. 20, p. 36 (cit. part.) (1904). - Fruhstorfer, Deutsch. Ent. Zeit. Iris, v. 16 (1903), p. $314,320(1904)$. - II. Stichel, Tierreicl, Lief. 25, p. 173 (cit. part.), f. 39 (1909). - Fruhstorfer: A. Seitz, Großschmett. Erde, v. 5, p. 319 (1912).

C. ilioneus (non Pap. i. Cram.), Hahnel, Deutsch. Ent. Zeit., Lep. Iris, p. 166 (1890).

subsp. s a $l t u s$ Kaye $C$. s., W. J. Kaye, Trinidad Trans. Ent. Soc. London, p. 165 (1904). Guppy, 1. c., p. 226 , t. 17 , f. $1,1 \mathrm{a}-\mathrm{f}$ (Metamorph.) (1904). - C. illioneus s., Fruhstorfer: A. Seitz, Großschmett. Erde, v. 5, p. 319 (1912). $-C$. ilioneus s., W. J. Kaye, Trans. Ent. Soc. London, p. 549 (1914).

C. i. polyxenus (in cit.), H. Stichel, Gen. Ins., Fasc. 20, p. 36 (1904). - Id., Tierreich, Lief. 25, p. 173 (1909).

subsp. oberon Butl. C. o., A. G. Butler, Panama, Trans. Ent. Soc. Iondon, p. 498 (part.) Colombia, (1870). - W. F. Kirby, Cat. Diurn. Iep., ?Ecuador p. $616(1871)$. - W. F. Kirby, Samml. Exot. Schmett., Hübner \& Geyer, Neue Ausg., v. 3 , p. 52 (1901). - C. illioners o., II. Stichel, Gen. Ins., Fasc. 20, p. 36 (cit. part.) (1904). - Fruhstorfer, Deutsch. Ent. Zcit. Iris, v. 16 (1903), p. 313, 320 (1904). - H. Stichel, Tierreich, Licf. 25, p. 173 (1909). - Fruhstorfer: A. Seitz, Großschmett. Erde, v. 5, p. 318 (1912).

Potamis conspicua teucer (non Pap. $t$. Linné), Jac. Hübner, Samml. Exot. Schmett., v. 1, t. [77] $(1806-19)$ - $-P$. Conspicua t., W. F. Kirby, 1. c., Neue Ausg., t. $77(1891-95)$. 
Caligo illioneu, Jac. Hübner, Verz. Schmett., p. 51 (cit. part.) (1818). - Id., Index Exot. Lep., p. [6] (1821). - Pavonia ilioneus Boisduval, Consid. Lép. Guatemala, p. 56 (sine cit.) (1870). - Caligo $i$., W. F. Kirby, Cat. Diurn. Lop., p. 128 (cit. part.) (1871). - Pavonia i., H. W. Bates, Proc. Zool. London, p. 249 (1873). - Caligo i., W. F. Kirby, Cat. Coll. Diurn. Lep. Hewitson, p. 110 (part.) (1879). - F. D. Godman \& O. Salvin, Biol. Centr.-Amer., Lep.-Rhop., v. 1, p. 133 (part.) (1881). O. Staudinger (\& Schatz), Exot. Schmett., v. 1, p. 216 (part.) (1887). - ? Dognin, Lép. Loja, p. 34 (1898). - Therese v. Bayern, Berlin. Ent. Zeit., v. 46, (1901), p. 265 (distrib. part.) (1902). - Fassl, Fauna Exot., v. 1, p. 26 (1911). - Id., Ent. Rundschau, v. 31, p. 37 (1914). - Id., 1. c., v. 35 , p. 31 (1918).

subsp. praxs iodus Fruhst. C. illioneus p., Peru

Fruhstorfor: A. Seitz, Großschmett. Erde, v. 5 , p. 318 (1912).

? C. ilioneus, H. G. Dyar, Proc. U. S. Nat. Mus, Washington, v. 45 , p. 636 (1913).

subsp. pheidriades Fruhst. C. illioneus Bolivia p., Fruhstorfer: A. Seitz, Großschmett. Ende, v. 5, p. 319 (1912).

? C. ilioneus, W. F. Kirby, Cat. Coll. Diurn. Lep. Howitson, p. 110 (part.) (1879).

subsp. pampeiro Fruhst. C. illioneus p., Paraguay

Fruhstorfor, Soc. Ent., v. 18, p. 145 (1904).

- Id., Dentsch. Ent. Zeit. Iris, v. 16 (1903), p. 313, 320 (1901). - H. Stichel, Gen. Ins., Fasc. 20, p. 36 (1904). - Id., Tierreich, Lief. 25, p. 173 (1909). - Fruhstorfer: A.

Seitz, Großschmett. Erde, v. 5, p. 319 (1912).

- C. ilionsus p., W. Rothschild, Nov. Zool., p. 312 , t. 5, f. 1 (larva) (1916).

- prometheus (Koll.) Morpho p., Kollar, Denkschr. Colombia, Ak. Wissensch. Wien, v. 1, p. 357 (1849). Ecuador

- Caligo p., H. Stichel, Gen. Ins., Fasc. 20,

p. 36 (1904). - Id., Tierreich, Lief. 25, p. 175 (1909). - Frulstorfer: A. Seitz, Großschmett. Fride, v. 5, p. 320 (1912).

C. memnon (part.), W. J. Kaye, Proc. Ent. Soc. London, p. XIX (1917).

subsp. prometheus (Koll.) Morpho p., Colombia

Kollar, ut antea, t. 43, f. 3, 4 (1849). -

Caligo $p$., Herrich-Schäffor, Corr.-Bl. Zool.-

Min. Ver. Regensb., v. 19, p. 65 (1865). -

W. F. Kirby, Cat. Diurn. Lep., p. 128 (1871). - C. Crüger, Verh. Vor. Naturw. Unterh. Hamburg, v. 4 (1877), p. 193 (1879). - O. Staudinger (\& Schatz), Exot. Schmett., v. 1, p. 216 (1887). - Woymer (\& Maa(Ben), Lep. Reise Stübel, p. 17 (1890). Fruhstorfer, Deutsch. Ent. Zeit. Iris, v. 16 (1903), p. 319 (1904). - C. p. p., H. Sti- 
chel, Ins.-Börse, v. 21, p. 212 (1904). - Id., Gen. Ins., Fase. 20, p. 36 (1905). - Fruhstorfer, Ent. Zeit. Stettin, v. 68, p. 130 (1907). - H. Stichel, Tierreich, Lief. 25, p. 177 (forma principalis) (1909). - Fruhstorfer: A. Seitz, Großschmett. Ende, v. 5, p. 320 (1912). - C. p., Fassl, Entom. Rundschau, v. 30 , p. 15 (1913). - C. memnon p., W. J. Kaye, Proc. Ent. Soc. London, p. XXI (1917). - O. p., Fassl, Ent. Rundschau, v. 35 , p. 31 (1918). - Giacomelli, Rev. Chil. Hist. Nat., v. 27, p. 16 (1924). - Schwanwitsch, Proc. Zool. Soc. London, p. 521, 522, t. 3, f. 18 (Morphol.) (1924).

C. dardanus (?) (non Pavoniad. Boisduval), F. D. Godman \& O. Salvin, Biol. Centr.Amer., Lep.-Rhop., จ. 1, p. 134 (1881).

forma epimetheus (Feld.) Pavonia e., Typ.: C. \& R. Felder, Reise Novara, จ. 2. II, p. Colombia 455 (1866). - Caligo e., T. F. Kirby, Cat. Diurn. Lep., p. 128 (1871). - C. e., O. Staudinger (\& Schatz), Exot. Schmett., v. 1, p. 216 (prometheus-Form) (1887). C. p. e., H. Stichel, Ins.-Börse, v. 21, p. 212 (part.) (1904). - Id., Gen. Ins., Fasc. 20, p. 36 (excl. cit. C. phorbas) (1904). $-C$. p. $p$. forma e., id., Tierreich, Lief. $25, \mathrm{p}$. 177 (1909). - C. e., Fassl, Soc. Ent., v. 24, p. 115 (Metamorph.) (1909). - C. p. e. (Talform), Frulistorfer: A. Seitz, Großschmett. Eivde, v. 5, p. 320 (1912). - C. e., Fassl, Tnt. Rundschau, v. 31, p. 44 (1914). Id., 1. c., v. 33 , p. 26 (1916) - C. prometheus e., IV. Rothschild, Nor. Zool., v. 23, p. 313, t. 5, f. 2 (larva) (1916). C. memnon $p$. var. e., W. J. Kaye, Proc. Ent. Soc. London, p. XX, XXI (1917).

C. at las (non Röber), Fruhstorfer, Deutsch. Ent. Zeit. Iris, v. 16 (1903), p. 319 (in cit.) (1904).

$H$ änschi Röb. C. h., Röber, Soc. Entom., v. 18, p. 146 (1904). - C. haenschi, id., l. c., v. 21, p. 28 (1906). - C. prometheus $p$. forma $h$., Fruhstorfer, Ent. Zeit. Stettin, v. 68 , p. 130 (1907).

subsp. a $t$ la \& Röb. C. a., Röber, Soc. Ent., v. Ecuador 19, p. 146 (1904). - H. Stichel, Gen. Ins., Fasc. 31, p. 16 (corr. ad Fasc. 20, p. 36) (1905). - Röber, Soc. Ent., v. 21, p. 28 (1906). - C. epimetheus a., Fruhstorfer, Ent. Zeit. Stettin, v. 68, p. 130 (1907). - H. Stichel, Soc. Ent., v. 22, p. 100 (1907). - C. prometheus a., id., Berlin. Ent. Zeit., v. 52, p. 162 (1908). - Id., Tierreich, Lief. 25, p. 178 (1909). - Fruhstorfer: A. Seitz, Großschmett. Erde, v. 5, p. 320 (1912). C. memnon a., TV. J. Kaye, Proc. Ent. Soc. London, p. XX, XXI (1917). 
? C. teucer var. prometheus, Dognin, Lép. Loja, p. 34 (1891).

? C. me mnon, Dognin, Lép. Loja; p. 72 (1894).

C. prometheus epimetheus (non Pavonia $e$. Felder), Fruhstorfer, Deutsch. Ent. Zeit. Iris, v. 16 (1903), p. 316, 319 (1904). - C. p. e., H. Stichel, Ins.-Börse, v. 21, p. 212 (part.) (1904). - Id., Gen. Ins., Fase. 20 , p. 36 (in cit. falso C. phorbas, conf. corr. in Fase. 20, ut antea) (1904).

? C. t el a monius, Campos, Rev. Col. Nacion. Vivente Rocafuerte, Nr. 4, p. 30 (1921).

subsp. a $n a x$ imandrus Fruhst. C. p. a., OstFruhstorfer, Ent. Rundschau, v. 29, p. 16 Colombia (1912). - Id., A. Seitz, Großschmett. Erde, v. 5, p. 320 (1912). - Fassl, l. c., v. 33, p. 26 (1916). - C. memnon (?) a., IV. J. Kaye, Proc. Ent. Soc. London, p. XX, XXI (1917). - C. a., Fassl, Ent. Rundschau, v. 35 , p. 31 (1918).

- memnon (Feld.) Pavonia m., C. \& R. Felder, Reise MexikoNovara, v. 2. II, p. 454 (1866). - Caligo m., H. Stichel, Gen. Ins., Fasc. 20, p. 36 (1904). - Id., Tierreich, Licf. 25, p. 178 (1909). - Fruhstorfer: A. Seitz, Großschmett. Erde, v. 5, p. 320 (1912).

C. teue er (non Pap. t. Linné) (part.), W. J. Kaye, Proc. Ent. Soc. London, p. XX, XXI (1917).

subsp. $m$ e $m$ n on (Feld.) Pavonia m., O.\& R. MexikoFelder, ut antea (1866). - Caligo m., W. Costa Rica F. Kirby, Cat. Diurn. Lep., p. 128 (1871). - A. G. Butlor \& Jerb. Druce, Proc. Zool. Soc. London, p. 339 (1874). - F. D. Godman \& O. Salvin, Biol. Centr.-Amer., Lep.Rhop., v. 1, p. 133 (excl. cit. C. taramela), t. 14, f. $1,4(1881)$. - 0 . Staudinger (\& Schatz), Exot. Schmett., v. 1, p. 216 (1887). - Tristan, Ins. Costa Rica, p. 17 (1897). A. G. Butler, Entomologist, จ. 33, p. 190 (1900). - C. m. m., Fruhstorfer', Deutsch. Ent. Zeit. Iris, v. 16 (1903), p. 319 (1904). - H. Stichel, Ins.-Börse, v. 21, p. 212 (1904). - Id., Gen. Ins. Fasc. 20, p. 36 (1904). - C. m., A. Hall, Entomologist, v. 38 , p. 230 (1905). - C. m. m., H. Stichel, Tierreich, Lief. 25, p. 180 (1909). - Fruhstorfer: A. Seitz, Großschmett. Ende, v. 5, p. 320 , t. 65 a (1912). - C. m, Gibbs, Proc. Ent. Soc. London, p. XLVI, XLVIII (1912). - Davis, Trans, Ent. Soc. Iondon, p. 198, t. 29 (Metamorph.) (1915). - C. m. m., W. J. Kaye, Proc. Ent. Soc. London, p. XXI (1917). - C. m., Strand, Sac. Ent., v. 33, p. 19 (1918).

dardanus (Boisd.) Pavonia d., Boisduval, Consid. Lép. Guatemala, p. 56 (Q) (1870). - Caligo d., W. F. Kirby, Cat. Diurn. Lep. 
App., p. 646 (1871). - F. D. Godman \& O. Salvin, Biol. Centr.-Amer., Lep.-Rhop., v. 1, p. 134 (? = prometheus) (1881). forma $c a \in h i$ Joicey \& Kaye C. teucer c., Typ.: Joicey \& W. J. Kaye, Ent. Ree. Journ. Var., v. 29, p. 181 (1917). - W. J. Kaye, Proc. Ent. Soc. London, p. XX, XXI (1917). (Forma incertao sodis).

? C. telamonius (non Pavonia $t$. Felder), Boisduval, Consid. Lép. Guatemala, p. 57 (part.) (1870). - C. t., F. D. Godman \& O. Salvin, Biol. Centr.-Amer., Lep.-Rhop., v. 1, p. 134 (part.), t. 14, f. 1,2 (1881).

? C. promethe us (non Morpho p. Kollar), A. G. Butler, Entomologist, v. 33, p. 190 (1900).

C. me m non menes (non Fruhstorfer), H. Stichel, Tierreich, Lief. 25, p. 181 (part.) (1909).

subsp. menes (Fruhst.) C. m. menus (lap- Panama sus, corr. 1904: menes), Frulistorfer, Ins.Börse, v. 20, p. 413 (1903). - C. memnon menes, id., Deutsch. Int. Zeit. Iris, v. 16 (1903), p. 317, 319 (1904). - H. Stichel, Ins.-Börse, v. 21, p. 212 (part.) (1904). C. memnon menes (C. telamonius ab. livoris Staudinger \& Bang-Haas i. 1.), id., Gen. Ins., Fasc. 20, p. 37 (1904). - Id., Tiorreich, Lief. 25, p. 181 (forma principalis) (cit. part.) (1909). - Fruhstorfer: $\Lambda$. Seitz, Großschmett. Erde, v. 5, p. 321 (1912). - C. teucer menes, WV. J. Kaye, Proc. Ent. Soc. London, p. XX, XXI (1917).

c. te la mon i us, Boisduval, Consid. Lép. Guatemala, p. 57 (part.) (1870). - F. D. Godman \& O. Salvin, Biol. Centr.-Amer., Lep.Rhop., v. 1, p. 134 (part.) (non t. 14, f. 1, $2:=$ forma eaehi) (1881). - O. Staudinger (\& Schatz), Exot. Schmett., v. 1, p. 216 (part.) (1887).

forma $d i l u t a$ Stich. C. memnon mencs forma Typ.: d., II. Stichel, Tierreich, Lief. 25, p. 182 Chiriqui (1909). - C. memnon d., Fruhstorfer: A. Seitz, Großschmett. Erde, v. 5, p. 320 (1912).

subsp. pe le us Stich. C. m. p., H. Stichel, Venezuela Gen. Ins., Fasc. 20, p. 37 (1904). - Id., Tierreich, Licf. 25 , p. 182 , f. 40 (1909). Fruhstorfer: A. Seitz, Großschmett. Erde, v. 5, p. 321 (1912). - C. teucer p., W. J. Kaye, Proc. Ent. Soc. London, p. XX, XXI (1917).

? C. te lam on $i u s$, Boisduval, Consid. Lép. Guatemala, p. 57 (part.) (1870).

subsp. te lamonirs (Feld.) Pavonia t., C. Colombia, \& R. Foldor, Wien. Ent. Monatschr., v. 6, Westp. 422 (1862). - Iid., Reise Novara, v. 2. Venezuela II, p. 454 (part.) (non t. 64, f. $1:=$ forma pavo) (1866). - Caligo t., Herrich-Schäffer, Corr.-B1. Zool.-Min. Ver. Regensb., v. 
19, p. 65 (1865). - Pavonia t., Boisduval, Consid. Lép. Guatemala, p. 57 (part.) (1870). - Caligo t., W. F. Kirby, Cat. Diurn. Lep., p. 128 (1871). - Möschler, Verh. Zool.-Bot. Ges. Wien, v. 26, Abh., p. 321 (1877). Pavonia t., IV. F. Kirby, Cat. Coll. Diurn. Lep. Hewitson, p. 110 (1879). - Caligo $t$., F. D. Godman \& O. Salvin, Trans. Ent. Soc. London, p. 122 (1880). - Tid., Biol. Centr.-Amor., Lep.-Rhop., v. 1, p. 134 (part.) (non t. 14, f. 1, 2: = forma cachi) (1881). - O. Staudinger (\& Schatz), Exot. Schmett., v. 1 , p. 216 (part.) (1887). - L. Glaser, Cat. Etymol. Col. Lep., p. 394 (1887). Röber: O. Staudinger \& Schatz, Exot. Schmett., v. 2, p. 194 (1889). - C. memnon t., Fruhstorfer, Deutsch. Ent. Zeit. Iris, v. 16 (1903), p. 319 (1904). - C. t., C. m. t.,

H. Stichel, Ins.-Börse, v. 21, p. 212 (1904).

- C. m. t., id., Gen. Ins., Fase. 20, p. 37 (cit. part.) (1904). - Id., Tierreich, Lief. 25 , p. 184 (forma principalis) (1909). ? C. t., A. G. Weeks, Illustr. Diurn. Lep., v. 2, p. XI (1911). - C. memnon t., Fruhstorfer: A. Seitz, Großschmett. Erde, v. 5, p. 321 (1912). - C. teucer telamonius, W. F. Kaye, Proc. Ent. Soc. London, p. XX, XXI (1917). - ? C. t., Fassl, Ent. Rundschau, v. 35, p. 31 (1918).

? C. memnon (non Pavonia m. Felder), M. Hering, Biol. Schmett, p. 51 (not. biol. sec. IV. Hopp) (1926).

forma $p a v o$ Röb. C. $p$, Röber, Soc. Ent., v. Typ.:

18, p. 145 (1904). - C. m. p., Fruhstorfer, Colombia Deutsch. Ent. Zeit. Iris, v. 16 (1903), p. 319 (1904). - C. p., Röber, Soc. Ent., v. 21, p. 28 (1906). - C. memnon telamonius forma p., H. Stichel, Soc. Ent., v. 22, p. 100 (1907). - Id., Tierreich, Lief. 25, p. 184 (1909). C. m. p., Fruhstorfer: A. Seitz, Großschmett. Erde, v. 5, p. 322 (Thulform) (1912). ? Rosa, Bol. Mus. Nacion. Rio de Janeiro, v. 2, Nr. 4, p. 24 (1926).

C. $m$. $m$ en e s, H. Stichel, Ins.-Börse, v. 21, p. 212 (part.) (1904). - Id., Gen. Ins., Fasc. 20, p. 37 (part.) (1904).

C. telam on $i u s$, C. \& R. Felder, Reise Novara, v. 2. II, p. 454 (part.), t. 64, f. 1 (1866). - W. J. Kaye, Proc. Ent. Soc. London, p. XX (1917).

subsp. $p$ a vonides Fruhst. C. M. p., Fruh- Oststorfer: A. Seitz, GroBschmett. Erde, v. 5, Colombia p. 322 (1912). - C. m. var. p., Fassl, Ent. (Rio Negro Rundschau, v. 33, p. 26 (1916). - C. p. sup.) (? C. tencer forma), W. T. Kaye, Proc. Ent. Soc. London, p. XX (1917). - C. p., Fassl, Ent. Rundschau, v. 35, p. 31 (1918).

bellerophon Stich. C. b. (Pavonia b. Maassen MS), Ecuador

H. Stichel, Ins.-Börse, v. 20, p. 389 (1903). 
- Fruhstorfer, Deutsch. Ent. Zeit. Iris, v. 16 (1903), p. 319 (1904). - H. Stichel, Gen. Ins., Fasc. 20, p. 37, t. 5, f. 2 (1904). - Id. Tierreich, Lief. 25, p. 181 (1909). - Fruhstorfer: A. Seitz, Großschmett. Erde, v. 5, p. 322, t. 63 a (1912).

eurilochus (Cram.) Papilio e., P. Cramer, Pap. Nördliches Exot., v. 1, p .152 (1776). - Caligo e. (part.) Süd-

H. Stichel, Gen. Ins., Fasc. 20, p. 37 (1904). Amerika

- Id., Tierreich, Lief. 25, p. 186 (1909). bis

- Fruhstorfer: A. Seitz, Großschmett. Er- Bolivia de, v. 5, p. 322 (1912). - C. eurylochus, W. Rothschild, Nov. Zool., v. 23, p. 313 (1916).

- subsp. curilochus (Cram.) -, Seba, The- Guayana, saurus, v. 4 , p. 38, t. 31, f. 3,4 (sec. Auririvillius, Recens. crit., $1882,=C$. teucer, sed errans puto); ? p. 51, t. 43 , f. 15,16 (1765). - "Eurilochus", Papilio e., P. Cramer, ut antea, p. 53 , t. 33 , f. A; t. 34 , f. A; p. 152 (1775 \& 76). - P. (Eques Achivus) e., Goeze, Entom. Beytr., v. 3. I, p. 79 (1779). - P. e., C. Stoll, Essai, Syst. Lep. (P. Cramer, Pap. Exot., v. 4, App.), p. 8, Nr. 16 (1782). - P. (Eques Achivus) eurylochus, [D. H. Schneider], Nomencl. Entom., p. 33 (1785). - P. e., (Jablonsky \&) Herbst, Naturs. Ins., Schmett., v. 3, p. 67, 71, t. 29 , f. 1,2 (Q) (1788). - P. (Eques) teucer, B. $P$. eurylochus, Gmelin-Linné, Syst. Nat., ed. 13 , p. 2248 (part.) (1790). - P. eurilochus, Jung, Alphab. Verz. Schmett., v. 1, p. 205 (1791). - P. eurilochus, Tableau Encycl. Méth., v. 18, t. 25, f. 1, 1 bis (1797). - Esper, Ausl. Schunett., p. 182, t. 44 , f. 3 (O); ed. 2, ibid. (1801 \& 30). - Caligo euriloche, Jac. Hübner, Verz. Schmett., p. 51 (1818). - Morpho eurylochus, Pavonia e., (Latreille \&) J. B. Godart, Enc. Méth., v. 9, p. 448, 807 (1824). - Potamis euriloche, Jac. Hübner, Cat. Lép. Franck, p. 79 [1826]. - Pavonia eurylochus, Verloren, Cat. Ins. Lep. Cramer, v. 1, p. 27; v. 2, p. 202 (1837). - E. Doubleday, List Lep. Brit. Mus., v. 1, p. 117 (1844). - Erichson: Schomburgk, Guiana, v. 3, p. 599 (1848). Morpho e., Gistel, Naturg. Thierneich, p. 145 (1848). - Caligo e., (E. Doubleday \&) J. O. Westwood, Gen. Diurn., v. 2, p. 342 (1851). - Chenu \& H. Lucas, Encycl. Hist. Nat., Pap. Diurn., p .168 (1851-53). - Ménétriés, Enum. Corp. Anim. Ac. Petropol., Lep., pars 1, p. 38 (1855). - A. Fauvel, Bull. Soc. Linn. Normand., v. 6 (1860-61), p. 139 (1862). - C. eurilochus, Herrich-Schäffer, Corr.-Bl. Zool.-Min. Ver. Regensb., v. 19, p. 65 (1865). - C. eurylochus, W. F. Kirby, Cat. Diurn. Lep., p. 127 (part.) (1871). - C. eurilochus, J. G. Wood, Insects abroad, p. XII, 595, t. 
14 (fig. mal.) (1874). - C. eurylochus, Möschler, Verh. Zool.-Bot. Ges. Wien, v. 26, Abh., p. 321 (1877). - Pavonia e., W. F. Kirby, Cat. Coll. Diurn. Lep. Hewitson, p. 110 (part) (1879). - C. e., F. D. Godman \& O. Salvin, Biol. Centr-Amer., Lep.-Rhop., v. 1, p. 131 (part.) (1881). - C. e., Pavonia e., L. Glaser, Cat. Etymol. Col. Lep., p. 278, 306, 368 (1887). - C. e., O. Staudinger (\& Schatz), Exot. Schmett., v. 1, p. 215 (part.) (1887). - Röber, 1. c., ₹. 2, p .190, f. 1-3 (Metamorph.) [? spec. haec. aut C. brasiliensis!]; p. 193, 194 (1889). - W. F. Kirby, Handb. Lep., v. 1, p. 200 (1894). - C. eurilochus, II. Stichel, Ins.-Börse, v. 20, p. 389 (1903). $\rightarrow$ C. e. e., id., Gen. Ins., Fasc. 20, p. 37 (1904). Fruhstorfer, Deutsch. Ent. Zeit. Iris, v. 16 (1903), p. 315, 319 (sine cit.) (1904). C. e., Silva, Relat. Contrib. Hist. Nat. Lep. Brasil (Congr. Scient. Lat.-Amer., 1905, v. 3B), p. 91 (part.) (1907). - C. e. e., H. Stichel, Tierreich, Lief. 25, p. 189, f. 41 (1909). - Fruhstorfer: A. Seitz, Großschmett. Erde, v. 5, p. 323 (1912). - C. eurylochus e., W. Rothschild, Nov. Zool., v. 23, p. 313 (1916). - C. e., Ealand, Ins. Life, p. 145 (1921). - ? Giacomelli, Rev. Chil. Hist. Nat., v. 27, p. 16 (1924). - A. Seitz, Ent. Rundschau, v. 45, p. 31 (ovum) (1928).

? "Teucer" var., Meuschen \& Boddaert, Mus. Gronovian., p. 70, Nr. 679 (1778).

? C. idomeneus, Palisot, Ins. Afr. Amér., p. 220, t. 6, f. 1 a, b (fig. dub., vide quoque c. idomeneus $i$. ipso!) (1805).

subsp. $l i$ v $i$ u s Stauding. C. l., O. Staudinger Colombia,

(\& Schatz), Exot. Schmett., v. 1, t. 74; p. Peru,

215 (part.) $(1886$ \& 87). - C. eurilochus l., West-

H. Stichel, Gen. Tns., Fasc. 20, p. 37 (part.) Amazonas

(1904). - Id., Tierreich, Lief. 25, p. 191

(cit. part.) (1909). - Fruhstorfer: A. Seitz,

Großschmett. Erde, v. 5, p. 322 (part.)

(1912). - C. curylochus l., W. Rothschild, Nov. Zool., v. 23, p. 313 (1916).

C. eurilochus e., Fruhstarfer, Deutsch. Ent. Zeit. Lep. (Iris), v. 16 (1903), p. 315, 318 (part. in cit.) (1904).

andicolens Stich. C. eurylochus a. (Pavonia $a$. Felder MSS), H. Stichel, Ins-Börse, v. 20 , p. 389 (1903). - Id., Gen. Ins., Fasc. 20 , p. 38 (1904).

subsp. de le ctans Joicey \& Kaye C.e.d., Venezuela Joicey \& W. J. Kaye, Ent. Rec. Journ. Var., v. 29, p. 181 (1917). (Subsp. aut forma incertae sedis, ? =C. brasitiensis caesia).

? C. eurylochus var., O. Staudinger (\& Schatz), Exot. Schmett., v. 1, p. 216 (1887). 
- ? C. e., Hahnel, Deutsch. Ent. Zeit. Lep. (Tris), v. 3, p. 145 (1890).

? C. e. livius, Fruhstorfer: A. Seitz, Großschmett. Erde, v. 5, p. 322 (part.) (not. biol. sec. Hahnel, 1890).

subsp. pallida Fruhst. C. e. pallidus, Fruh- Bolivia storfer, Ins.-Börse, v. 21, p. 125 (1904). Id.: A. Scitz, Großschmett. Erde, v. 5, p. 323 (1912). - C. eurylochus p., W. Rothschild, Nov. Zool., v. 23, p. 313 (1916).

C. e. livi \& (non C. l. Staudinger), H. Stichel, Gen. Ins., Fasc. 20, p. 37 (in cit.) (1904). - Id., Tierreich, Lief. 25, p. 191 (in cit.) (1909).

- brasiliensis (Feld.) Pavonia eurylochus var. b., C. Mittel-, Felder, Verh. Zool.-Bot. Ges. Wien, v. 12, SüdAbh., p. 476 (1862). - Caligo b., W. Roth- Amerika schild, Nov. Zool., v. 23, p. 313 (1916).

C. eurilochus (non Papilio e. Cramer), (part.), II. Stichel, Gron. Ins., Fasc. 20, p. 37 (1904). - Id., Tierreich, Lief. 25, p. 186 (1909). - Frulistorfer: A. Seitz, Großschmett. Erde, v. 5, p. 322 (1912).

subsp. brasiliensis Feld. Pavonia eurylochus val. $b$., C. Felder, ut antea (1862). $P$. eurylacchus (!) var. b., $P$. eurylochus var. b., E. Deyrolle, Rov. Mag. Zool., ser. 2, v. 23, p. 19,64 (part.) (1872), - Caligo eurilochus b., Frulistorfer, Deutsch. Ent. Zeit. Iris, v. 16 (1903), p. 315,319 (1904). - H. Stichel, Gen. Ins., Fasc. 20, p. 38 (1904). - Id., Tierreich, Lief. 25, p. 193 (1909). - Fruhstorfer: A. Seitz, Großschmett. Erde, v. 5, p. 323 (1912). - C. $b$. b., W. Rothechild, Nov, Zool., v. 23, p. 313, t. 5, f. 9, 10 (larva, chrysal.) (1916) - C. eurylochus b., Ferreira d'Almeida, Mél. Lépid., p. 100 (Biol.) (1922). - C. e.-br., Zikán, Ent. Rundschau, v. 45, p. 11 (1928). C. eurilochus b., F. Hoffinann, Zeit. wiss. Ins.-Biol., v. 25, p. 95 (ovum, not. biol.) (1930). - Zikán, Ent. Rundschau, v. 47, p. 33 (not. theoret.) (1930).

C. ilioneus (non Papilio illioneus Cram.), H. A. Hagen, Proc. Boston Soc. Nat. Hist., v. 12, p. 163 (Monstros.) (1868).

C. e urylochus, W. F. Kirby, Cat. Diurn. Lep., p. 127 (part.) (1871). - H. A. Hagen, Ent. Zeit. Stettin, v. 33, p. 398 (Monstros.) (1872). - Pavonin e., Burmeister, Rev. Mag. Zool., ser. 3, v. 1, p. 36, t. 4 (Metanorpl..) (1873). - Capronnier, Ann. Soc. Ent. Belg., v. 17, p. $2 \mathrm{~s}$ (Phrenol.) (1874). - Caligo e., H. A. Hageu, Mem. comp. Zool. Harvard Coll., v. 2, Nr. 9, p. 9, t. 1, f. $1-4$ (Monstros. ut 1868 \& 72) (1876). - Burmeister, Descr. Phys. Argent., v. 5. II, p. 20, t. 16 (Metamorph.) (1879). - Capron-

\author{
Mittel-,
Süd-
Brasilien,
Argentinie \\ Mittel-,
Süd-
Brasilien,
Argentinie \\ Mittel-,
Süd-
Brasilien,
Argentinie \\ Mittel-,
Süd--
Brasilien,
Argentinien
}

bis 

nier, Ann. Soc. Ent. Belg., v. 25, p. man \& O. Salvin, Biol. Centr.-Amer., Lep.Rhop., v. 1, p. 131 (part. in eit) (1881). - G. W. Müller, Zool. Jahrb. Syst., v. 1, p. 597 (Motamorph.) (1886). - O. Staudinger (\& Schatr), Exot. Sehmett., v. 1, p. 215-16 (part.) (1887). - Weymer (\& Maassen), Lop. Reiso Stübel, p. 90 (1890). - Sommerfeld, Ins.-Börso, v. 13, p. 56 (not. biol.) (1596). - Bönninghausen, Verh. Ver. Naturw. Unterl. Hamburg, v. 9 (1894-95), p. 37 (1896). - C. eurilochus, Silva Relat. Contrib. Hist. Nat. Lep. Brasil (Congr. Scient. Lat.-Anıer., 1905, v. 3B), p. 91 (distr. part.), t. 16, f. 57 (fig. mala) (1907).

forma privata Truhst. C. e. forma $p$. 'Typ.:

Fruhstorfer: A. Seitz, Großschmett. Erde, v. Wispirito 5, p. 323.

C. eurilochus brasiliensis ab., Fruhstorfor, Deutsch. Ent. Zeit. Iris, v. 16 (1903), p. 315 (1904).

- subsp. morpheus Stich. C. eurilochus m., Zentral-,

II. Stichel, Ins.-Börse, v. 20, p. 389 (1903). Süd-

- Fruhstorfer, Deutsch. Ent. Zoit. Iris, v. Colombia

16 (1903), p. 315, 318 (1904). - H. Sti- bis Peru

chel, Gen. Ins., Fasc. 20, p. 38 (1904). -

Id., Tierreich, Liof. 25, p. 196 (1909). -

C. brasiliensis m., IV. Rothschild, Nov. Zool., v. 23, p. $313(1916)$. $-C$. $m$., Fassl, Ent. Rundschau, v. 35, p. 31 (1918).

O. e urylochus, (non Papilio eurilochus Cram.), Weymer (\& Marssen), Lep. Reise Stübel, p. 62 (sine cit.) (1890). - ? C. eurilochus, Dognin, Lép. Loja, p. 72 (1894).

? C. galba (non Pavonia g. Deyrolle), Fassl, Ent. Rundschau, v. 30, p .16; v. 35, p. 31 $(1913 \& 18)$.

subsp. galba (Deyr.) Pavonia g., E. Dey- Nordrolle, Rev. Mag. Zool,, ser. 3, v. 2, t. 6, 7 Colombia (deser. nulla) (1874). - Caligo g., W. F. Kirby, Cat. diurn. Lop., Suppl., p. 717 (1877). - C. eurilochus g., Fruhstorfer, Deutsch. Ent. Zeit. Lep., v. 16 (1903), p. 315 (1904). - II. Stichel, Gen. Ins., Fase. 20, p. 38 (1904). - Id., Tiorreich, Lief. 25, p. 195 (1909). - Fruhstorfor: A. Seitz, Großschmett. Erde, v. 5, p. 322 (1912). C. brasiliensis g., WV. Rothschild, Nov. Zool., v. 23, p. 313 (1916).

Pavonia eurylochus var. brasiliensis, E. Deyrolle, Rev. Mag. Zool, ser. 2, v. 23 , p. 19 (part.) (1872).

Caligo eurylochus, F. D. Godman \& O. Salvin, Biol. Centr.-Amer., Lep.-Rhop., v. 1, p. 131 (part. in cit.) (1881). - O. Staudinger (\& Schatz), Exot. Schmett., v. 1, p. 215 (part.) (1887). 
subsp. c a $s$ ia Stich. $C$. eurilochus cacsius, Venezuela

H. Stichel, Ins.-Börse, v. 20, p. 389 (1903).

- Fruhstorfer, Doutsch. Ent. Zcit. Iris, v. 16 (1903), p. 315 (1904). - C. e. caesia, H. Stichel, Gen. Ins., Fasc. 20, p. 38 (1904). Id., Tierreich, Lief. 25, p. 192 (1909). Fruhstorfer: A. Seitz, Großschmett. Erde, v. 5, p. 323 (1912). - C. brasiliensis c., TV. Rotlischild, Nov. Zool., v. 23, p. 313 (1916).

? C. eurylochus, Hahnel, Deutsch. Ent. Zeit. Lep. (Iris), v. 3, p. 145 (not. biol.) (1890).

? C. livius (non Staudinger), A. G. Weeks, Mllustr. Diurn. Lep., v. 2, p. XI (1911).

subsp. minor Kaye $C$. curylochus var. m., Trinidad W. J. Kaye, Truns. Fnt. Soc. London, p. 165 (1904). - C. m., C. brasiliensis m., W. Rothschild, Nov. Zool., v. 23, p. 312, 313 (1916).

C. teucer insulanus H. Stichel, Tierreich, Lief. 25, p. 167 (error in cit.) (1909). - W. J. Kaye, Trans. Ent. Soc. Iondon, p. 548 (1914).

$p h r y a \& u s$ Fruhst. C. eurilochus p., Fruhstorfer, Ent. Rundschau, v. 29, p. 15 (1912). - Id.: A. Seitz, GroBschmett. Erde, v. 5, p. 322 (1912). - W. T. Knye, Trans. Ent. Soc. London, p. 548 (1914).

subsp. 8 u $l$ a $n$ a Fruhst. C. eqrilochus sulanus, MittelFruhstorfer, Soc. Ent., v. 18, p. 145 (1904). Amerika - Id., Deutsch. Ent. Zeit. Iris, v. 16 (1903), p. 315,318 (1904). - H. Stichel, Gen. Ins., Fasc. 20, p. 38 (1904). - Id., Tierreich, Lief. 25, p. 193 (1909). - Fruhstorfer: $\Lambda$. Seitr, Großschmett. Erde, v. 5, p. 322, t. $63 \mathrm{c}(1912)$. - C. brasiliensis s., TV. Rothschild, Nov. Zool., v. 23, p. 313 (1916).

? Pavonia eurylochus, Boisduval, Consid. Lép. Guatemala, p. 56 (sine cit.) (1870). - Caligo e., A. G. Butler \& Herb. Druce, Proc. Zool. Soc. London, p. 339 (sine cit.) (1874). - F. D. Godman \& O. Salvin, Biol. Centr.-Amer., Lep.-Rhop., v. 1, p. 131 (part. in cit.) (1881). - O. Staudinger (\& Schatz), Exot. Schmett., v. 1, p. 216 (part.) (1887). - ? H. G. Dyar, Proc. U. S. Nat. Mus., v. 47, p. 144 (1914).

- idomeneus (L.) Papilio (Eques) i., Linne, Syst. SüdNat., al. 10 , p. 464 (1758). - Caligo i. Amerika (part.), H. Stichel, Ins.-Börse, v. 21, p. 204 (1904). - Id., Gen. Ins., Fasc. 20, p. 38 (1904). - Id., Tierreich, Lief. 25, p. 199 (1909). - Fruhstorfer: A. Seitz, Großschmett. Erde, r. 5, p. 324, 325 (1912).

subsp. idomen e $s$ (L.) - Blankaart, Guayana, Schon-Burg Rups., p. 55, t. 18, 19 (1690). — ? Pará, Merian, Metam. Ins. Surinam, t. 60 (imago Amazonas

solum, non larva: spec. dubia, conf. Bur- (part.) meister, Abh. Naturf. Ges. Halle, v. 2, 1854,

bis

Argentinien 
Sitzgsber., p. 64: Morpho i.). - Petiver, Gazophyl. Nat. Art., v. 1, t. 28, f. 1 (1709 \& 67). - Knorr, Deliciae Nat., t. 77 (C 1), f. 1 (sec. Aurivillius, 1882). - "Papitio Atlas", Seba, Thesaurus, v. 4, p. 30, t. 24, f. 7, 8 (1765). - Papilio (Eques) idomeneus, Linné, ut antea $(1758)$. $-P$. $i$., Mus. Ludov. Ulr., p. 213 (1761). - o. Clerck, Icon. Ins., t. 20, f. 1 (1764). - P. (Eques) i., Linné-Houttyn, Natuurl. Hist., v. 1. XI, p. 217 (1766-69). - Linné, Syst. Nat., ed. 12, v. 1. II, p. 753 (1767). - "Idomeneus". Merian-Buchoz, Hist. Ins. Surinam, ed. 3, v. 2 , p. 49; v .1, p. 60, t. 60 (1771). - Pap. (Achivus) idomeus (!), P. L. S. Müller, Naturs. Linn6, v. 5. I, p. 579 (1774). $-P$. idomeneus, J. C. Fabricius, Syst. Ent., p. 459 (cit. part.) (1775). - (Gmelin \&) Christman, Onomatol. Med., v. 6, p. 82 (1775). "Idomeneus", Papilio i., P. Cramer, Pap. Exot., v. 1, p. 82, t. 52, f. B; p. 153 (1775\& 76). - P. (Eques Achivrs) i., Groeze, Ent. Beytr., v. 3. I, p. 67 (1779). $P$. i., J. C. Fabricius, Spec. Ins., V. 2, p. 26 (cit. part.) (1781). - C. Stoll, Essai Syst. Lep. (P. Cuamer, Pap. Exot., v. 4, App.), p. 8, Nr. 18 (part.) (1782). - P. (Eques Achivus) i., [D. H. Schneider], Nomencl. Entom., p. 33 (1785). - P. i., J. C. Fabricius, Mant. Ins., v. 2, p. 13 (cit. part.) (1787). - (Jablonsky \&) Herbst, Naturs. Ins., Schmett., $\nabla .3$, p. 70 (cit. part.), t. 30 , f. 2 (non f. $1:=$ C. illioneus) (1788). Gmelin-Linné, Syst. Nat., ed. 13, v. 1. V, p. 2248 (cit. part.) (1790). - Jung, Alphab. Verz. Schmett., v. 1, p. 283 (1791). - J. C. Fabricius, Ent. syst., v. 3. I, p. 88 (cit. part.); Index alphab., p. 123 (1793 \& 96). "Idomeneus", P. i., G. Shaw, Natural. Miscell., v. 5, t. 164; Index (1794). - P. i., Fr. Weber, Nomencl. Entom., p. 101 (1795). - Latreille, Hist. Crust. Ins., v. 3, p. 392 (1802). - Thunberg, Mus. Natur. Ac. Upsal., pars 23, p. 8 (sec. Aurivillius, 1882) (1804). - P Palisot, Ins. Afr. Amér., p. 220 , Lep. t. 6, f. $1 \mathrm{a}, \mathrm{b}$ (an C. eurilochus) (1805). - Turton, Gen. Syst. Nat. Linné, v. 3. II, p. 89 (1806). - Oken, Lehrb. Naturg., v. 1 , p. 746 (1815). - Latreille: Cuvier, Règno Anim., v. 3, p. 545 (1817). Caligo idomenea, Jac. Hübncr, Verz. Schmett., p. 51 (1918). - Acrodes idomeneus, Billberg, E. 51 . Ins., p. 79 (1820). - Morpho i., Pavonia i., (Latreille \&) J. B. Godart, Enc. Méth., v. 9, p. 449 (part.); p. 807 (1824). - P. i., Lacoldaire, Ann. Soc. Ent. France, v. 2, p. 396 (1833). - Verloren, Cat. Ins. Lep. Cramer, v. 1, p. 33 (part.); v. 2, p. 202 (part.) (1837). - E. Doubleday, List 
Lep. Brit. Mus., v. 1, p. 107 (1844). Erichson: Schomburgk, Guiana, v. 3, p. 599 (1848). - Caligo i., (E. Doubleday \&) J. O. Westwood, Gen. Diurn. Lep., v. 2, p. 342 (cit. part.) (1851). - Chenu \& H. Lucas, Enc. Hist. Nat., Pap. Diurn., p. 168 (185153). - Mrénétriés, Enum. Coıp. Anim. Ac. Petropol., pars 1, p. 38 (1855). - J. O. Westwood, Trans, Ent. Soc. London, n. ser., v. 4, p. 165 (1857). - A. Fauvel, Bull. Soc. Linn. Normand., v. $6(1860-61)$, p. 140 (1862). - Prittwitz, Ent. Zeit. Stettin, v. 26, p. 309 (1865). - Herrich-Schäffer, Corr.B1. Zool.-Min. Ver. Regensb., v. 19, p. 65 (1865). - A. G. Butler, Cat. Diurn. Lop. Fabricius, p. 39 (1869). - W. F. Kirby, Trans. Ent. Soc. London, p. 137 (1870). Papilio i. (Caligo), id., Irans. Ent. Soc. London, p. 137 (identif. typ. Linnaei) (1870). - C. i., id., Oat. Diurn. Lep., p. 127, Suppl., p. 717 (1871). - Pavonia i., Burmeister, Rev. Mag. Zwol., ser. 3, v. 1, p. 41 (figurae Merianae incertae) (1873). - Caligo $i$., Möschler, Verh. Zool.-Bot. Ges. Wien, v. 26, Abh., p. 321 (1877). - ? Pavonia i., W. F. Kirby, Cat. Coll. Diurn. Lep. Hewitson, p. 110 (1879). - Caligo jdomenues (!), J. H.: G. Jüger, Handwörterb. Zool., v. 1, p. 501 (1880). - C. idomeners, Chr. Aurivillius, Svenska Ak. Handl., n. ser., จ. 19, Nr. 5, p. 33 (1882). - C. i., Pavonia i., L. Glaser, Cat. Etymol. Col. Lep., p. 278, 306 (1887). $C$. i., O. Staudinger (\& Schatz), Exot. Schmett., v. 1, p. 215 (1887). - ? Weymer (\& Maassen), Lep. Reise Stübel, p. 43 (1890). - E. M. Sharpe, Proc. Zool. Soc. London, p. 566 (1890). - E. Reuter, Acta Soc. Sci. Fenn., v. 22 , p. 113 (palpus) (1896). C. i. i., C. i., Fruhstorfer, Deutsch. Ent. Zeit. Iris, v. 16 (1903), p. 317, 318 (1904). - C. i. i., H. Stichel, Ins.-Börse, v. 21, p. 204 (1904). - Id., Gen. Ins., Fasc. 20, p. 38 (1904). - Fruhstorfer, Ent. Zeit. Stettin, v. 68, p. 128 (1907). - H. Stichel, Tierreich, Lief. 25, p. 199 (forma principalis) (cit. nonnull. except.) (1909). - Fruhstorfer: A. Seitz, Großschinett. Erde, v. 5, p. 324 (1912). - C. idomineus (!), W. Rothschild, Nov. Zool., v. 23, p. 314 (1916).

Papilia teucer (non Linné), Meerburgh, Albeeld. Zeldz. Gewass., t. 29 (1775).

subsp. rhoetus Stauding. C. $i$. var. r., O. OstStaudinger (\& Schatz), Exot. Schmett., v. Amazonas, 1, t. 74; p. 215 (1886 \& 87). - C. i. r., Mittel-, Fruhstorfer, Deutsch. Ent. Zeit. Iris, v. Süd6 (1903), p. 318 (1904). - H. Stichel, Brasilien Ins.-Börse, v. 21, p. 204 (1904). - Id., (part.), Gen. Ins., Fase. 20, p. 39 (1904). - Fruh- auch storfer, Ent. Zieit. Stettin, v. 68, p. 129 Mato Grosso 
(1907). - H. Stichel, Tierreich, Lief. 25, p. 201 (forma principalis) (1909). - Frubstorfer, Ent. Zeit., v. 24, p. 194 (1910).

Morphoidomeneus, Burmeister, Abh. Naturf. Ges. Halle, v. 2, Sitz.-Ber., p. 58 (1854). - Id., Rev. Mag. Zool., ser. 3, v. 1, p. 41 (1873). - ? id., Descr. Phys. Argent., v. 5. II, p. 20 , t. 16 (Metamorph.) (1879).

forma marsus Stich. C. i.r., forma m., H. Sti- Typ.: Pará chel, Ins.-Börse, v. 21, p. 204 (1904). - Id., Gen. Ins., Fasc. 20, p. 39 (1904). - C. i. r., forma mars, Fruhstorfer, Ent. Zeit. Stettin, v. 68, p. 129 (1907). - C. i. r. forma marsus, H. Stichel, Tierreich, Lief. 25, p. 201 (1909). - C. i. m., Fruhstorfer: A. Seitz, Großschmett. Erde, v. 5, p. 324 (Nebenform) (1912).

subsp. a pollonidas Frubst. C. i. a., Fruh- Amazonas storfer: A. Seitz, Großschmett. Erde, v. (Obidos) 5, p. 325 (1912).

C. i. a pollo + C. i. nidas (!), D. Sharp, Zool. Record, v. 50 (1913), pars 12, Ins., p. 335 (1914).

subsp. idomenides Fruhst. C. idomeneus Peru, idomenides, Fruhstorfer, Deutsch. Ent. Zeit. ? Ecuador Iris, $\nabla .16$ (1903), p. 317, 318 (1904) II. Stichel, Gen. Ins., Fase. 20, p. 39 (1904). - Id., Berlin. Ent. Zoit., v. 52 (1907), p. 177 (1908). - Id., Tierroich, Lief. 25, p. 202 (1909). - Fruhstorfer: A. Seitz, Großschmett. Erde, v. 5, p. 324 (1912).

? C. i d om e ne us, Herb. Druce, Proc. Zool., Soc. London, p. 217 (1876). - ? Dognin, Lép. Loja, p. 72 (1894).

subsp. praeca na Stich. C. i. superba forma Süd-Peru $p_{.}$H. Stichel, Berlin. Ent. Zoit., v. 52 (1907), p. 176, 177 (1908). - Id., Tierreich, Lief. 25, p. 202 (1909). - Frubstorfer: $\Lambda$. Seitz, Grobschmett. Erde, v. 5, p. 325 (1912). $-C$. (i.) p., W. Rothschild, Nov.

Zool., v. 23, p. 314 (1916).

subsp. hi p p o lo chus Fruhst. C. i. h., Fruh- Bolivia storfer: A. Seitz, Großschmett. Erde, v. 5, p. 324 (1913).

subsp. i Fruhstorfer, Ent. Zeit., v. 24, p. 194 (1910). Geraes - Id.: A. Seitz, Großschmett. Erde, v. 5, p. 325 (1912).

subsp. ariphron Fruhst. C. i. a., Fruh- Espirito storfer: Ent. Zeit., v. 24, p. 191 (1910). - Santo, Id.: A. Seitz, Großschmett. Erde, v. 5, p. ? Santa 325 (1912).

? C. idom e ne u s, Prittwitz, Ent. Zeit. Stettin, v. 26, p. 309 (sine cit.) (not. biol.) (1865).

- superba Stauding. C. idomeneus var. superbus, O. Peru, Staudinger (\& Schatz), Exot. Schmett., v. Ecuador 1 , p. 215 (1887).

Lepidopterorum Catalogus 51. 
C. $i d$ omeneus (non Papilio i. L.) (part.), H. Stichel, Gen. Ins., Fasc. 20, p. 38 (1904). - Id., Tiemeich, Lief. 25, p. 199 (1909). - Fruhstorfer: A. Seitz, Großschmett. Erde, v. 5, p. 324 (1912).

subsp. \& थ p e $r$ b a. Stauding. $C$. idomeneus var. Süd-Peru superbus, O. Staudinger (\& Schatz), ut antea (1887). - C. i. superba, H. Stichel, Ins.Börse, v. 21, p. 204 (1904). - Id., Gen. Ins., Fasc. 20, p. 39 (1904). - Id., Berlin. Ent. Zeit., v. 52 (1907), p. 177 (1905). Id., Tierreich, Lief. 25, p. 202 (part.) (1909). - Fruhstorfer: 4 . Seitz, Großschmett. Erde, v. 5, p. 324 (1912). - C. 8., IV. Rothschild, Nov. Zool., v. 23, p. 314 (1916).

C. idomeneus agamemnon (part. in cit.), Fruhstorfer, Deutsch. Ent. Zeit. Iris, v. $16,(1903)$, p. $317(1904)$.

subsp. a g a me m n on Woym. C.a., Weymer Ecuador (\& Maassen), Lep. Reise Stübel, p. 62, 111 , t. 1, f. 3 (1890). - C. idomeneus a., H. Stichel, Ins.-Börse, v. 21, p. 204 (1904). - Fruhstorfer, Deutsch. Ent. Zeit. Iris, V. 16 (1903), p. 317 (sine cit.) (1904). - H. Stichel, Gen. Ins., Fasc. 20, p. 39 (1904). - Id., Tierreich, Lief. 25, p. 203 (1909). - Fruhstorfer: A. Seitz, Großschmett. Erde, v. 5, p. 325 (1912).

euphorbus (Fold.) Pavonia e., O. \& R. Felder, Wien. Nördliches Int. Monatschr., v. 6, p. 123 (1862).

C. idomeneus (non Papilio i. L.) (part.), H. Stichel, Gen. Ins., Fasc. 20, p. 38 (1904). - Id., Tierneich, Lief. 25, p. 196 (1909). - C. i. (part.) + C. menoefius, Fruhstorfer: A. Seitz, Großschmett. Eivle, v. 5, p. 324, 325 (1912).

subsp. cuphorbus (Feld.) - ? Aubenton, Guayana, Planches enlum. Hist. Nat., t. 96, f. 1, 2 Amazonas, (sec. Aurivillius, 1882) (1765). - Pavonia Oste., C. \& R. Felder, ut antea (1862). - Colombia, Caligo e., Herrich-Schäffer, Corr.-Bl. Zool.- Peru Min. Ver. Regensb., v. 19, p. 65 (1865). W. F. Kirby, Cat. Diurn. Lep., p. 127 (1871). - Pavonia e., E. Deyrolle, Rov. Mag. Zool., ser. 2, v. 23 , p. 64, t. 9, 11 (1872). Caligo e., Herb. Druce, Proc. Zool. Soc. London, p. 217 (1876). - Chr. Aurivillius Svenska Ak. Handl., n. ser., v. 19, Nr. 5, p. 33 (1882). - O. Staudinger (\& Schatz), Exot. Schmett., v. 1, p. 215 (1887). - Fruhstorfer, Doutscl. Ent. Zeit., Iris, v. 16 (1903), p. 318 (1904). - C. idomeneus $i$. forma e., H. Stichel, Ins.-Börse, v. 21, p. 204 (1904). - Id., Gen. Ins., Fasc. 20, p. 39 (cit. part.) (1904). - C. i. e., Fruhstorfer, Ent. Zeit. Stettin, v. 68, p. 128 (1907). - C. $i$. forma $e_{.}$H. Stichel, Berlin. Int. Zeit., v. 52 (1907), p. 177 (1908). - C. i. i. forma e., id., Tierreich, Lief. 25, 
p. 201 (1909). - C. i. e., Fruhstorfer: A. Seitz, Großschmett. Erde, p. 324 (1912). - ? C. e., Fassl, Ent. Rundschau, v. 35, p. 31 (1918).

"I llomeneus", Papilio i. var. O, C. Stoll: P. Cramer, Pap. Exot., v. 4, p. 20s, t. 390, f. A, B; p. 250 (1782). - Id., Essai Syst. Lep. (P. Cramer, Pap. Exot., v. 4, App.), p. 8, Nr. 18 (1782). - P. i., J. O. Fabricius, Mant. Ins., v. 2, p. 13 (part. in cit.) (1787). - P. i. 우 var. (Jablonslsy \&) Herbst, Naturs. Ins. Schnett., v. 3, p. 73 (1788). - P. i., J. O. Fabricius, Ent. syst., v. 3. I, p. 88 (part. in eit.) (1793). - Pavonia i. ㅇ var., Verloren, Cat. Ins. Lep. Cramer, v. 1, p. 33, 145; v. 2, p. 202 (1837).

strat onides Fruhst. C. i. s., Fruhstorfer: A. Seitz, Großschmett. Ende, v. 5, p. 324 (1912).

forma me noetius Stauding. C. m., O. Stau- Typ.: dinger (\& Schatz), Exot. Schmett., v. 1, p. 216 (1887). - C. idomeneus i. forma m., H. Stichel, Tierreich, Lief. 25, p. 201 (1909). - C. m., Fruhstorfer: A. Seitz, Großschmett. Erde, v. 5, p. 325 (cit. corrig.) (1912). - W. Rothschild, Nov. Zool., v. 23, p. 314 (1916).

subsp. aristophanes Fruhst. C. menoe- Bolivia tius a., Fruhstorfer: A. Seitz, Großschmett. Erde, v. 5, p. 326 (1912) (subsp. dubia).

\section{b. Cohors Arisbiformes}

H. Stichel, Gen. Ins., Fasc. 20, p. 39 (1904). - Id., Tierreich, Lief. 25, p. 204 (1909).

arisbc Hübn. C. a., Jac. Hübner, Samml. Exot. Mittel- u. Schmett., จ. 2, t. $72(1820-26)$. Süd-

Brasilien

subsp. $a r i s$ be Hübn. C. a., Jac. Hübner, ut Espirito antea (1820-26). - Potamis a., id., Cat. Lép. Santo Franck, p. 79 (1826). - Caligo a., (E. Doubleday \&) J. O. Westwood, Gen. Diurn. Lep., v. 2, p. 312 (1851). - Ménétriés, Enum. Corp. Anim. Ac. Potropol., Lep., pars 1, p. 38 (1855). - Herrich-Schäffer, Corr.-Bl. Zvol.-Mfin. Ver. Regensb., v. 19, p. 65 (1865). - W. F. Kirby, Cat. Diurn. Lep., p. 128 (1871). - Pavonia a., id., Cat. Coll. Diurn. Lep. Hewitson, p. 110 (1879). - Caligo a., id., Samml. Exot. Schmett. Hübner \& Geyer, Neue Ausg., v. 2, t. 285 (72) (1898). Fruhstorfer, Deutsch. Ent. Zeit. Tris, v. 16 (1903), p. 320 (1904). - H. Stichel, Gen. Ins., Fasc. 20, p. 39 (part.) (1901). - Id., Tierreich, Lief. 25, p. 204 (part.) (1909). - Fruhstorfer: A. Seitz, Großschmett. Erde, v. 5, p. 327, t. 65a, (part.) (1912). - C. a. 
a., W. Rothschild, Nov. Zool., v. 23, p. 314 (1916).

ta r a me la God. Morpho t., Pavonia t., (Latreille \&) J. B. Godart, Enc. Méth., v. 9, p. 450; 807 (1824). - Caligo t., (E. Doubleday \&) J. O. Westwood, Gen. Diurn. Lep., v. 2, p. 342 (1851). - Herrich-Schäffer, Corr.-Bl. Zool.-Min. Ver. Regensb., v. 19, p. 65 (1865). - W. F. Kirby, Cat. Diurn. Lep., p. 128 (1871). - O. Staudinger (\& Schatz), Exot. Schmett., v. 1, p. 217 (1887).

C. memnon (non Pavonia m. Feld.), F. D. Godinan \& O. Salvin, Biol. Centr.-Amer., Lep.-Rhop., v. 1, p. 133 (in cit.) (1881). subsp. fulgens Rothscl. C. $\alpha$. f., W. Roth- Rio de schild, Nov. Zool., v. 23, p. 314 (1916). Janeiro,

$C$. $a r i s b e$ (non Hübner), O. Staudinger (\& Sao Paulo Schatz), Exot. Schmett., v. 1, p. 217 (1887). - Bönninghauson, Verh. Ver. Naturw. Unterh. Hamburg, v. 9 (1894-95), p. 38 (1896). - W. F. Kirby, Samml. Exot. Schmett. Hübner \& Geyer, Neue Ausg., v. 3, p. 53 (non v. 2, t. 285) (1901). - H. Stichel, Gen. Ins. et Tierreich, ut antea (part.) (1904 \& 09). - Fruhstorfer, ut antea (part.) (1912). - Zikín, Ent. Ruudschau, v. 45, p. 11 (1928).

Oberthiirii (Deyr.) Pavonia oberthurii, E. Deyrolle, Colombia Rev. Mag. Zool., ser. 2, v. 23, p. 20 (1872). -Peru

subsp. Oberthürii (Deyr.) Pavonix ober- Colombia, thurii, E. Deyrolle, ut antea, t. 1 (1872). Ecuador - Caligo o., W. F. Kirby, Cat. Diurn. Lep., Suppl., p. 717 (1877). - O. Staudinger (\& Schatz), Exot. Schmetto, v. 1, p. 217 (part.) (1887). - Dognin, Lép. Loja, p. 34 (1891). - C. oberthürii, H. Stichel, Gen. Ins., Fasc. 20 , p. 39 (part.) (1904). - Id., Tierreich, Lief. 25 , p. 206, f. 42 (part.) (1909). C. oberthïri, Fassl., Soc. Ent., v. 24, p. 115 (ovum, larva, not. biol.) (1909). - C. oberlhuri, Fruhstorfer: A. Seitz, Großschmett. Erde, v. 5, p. 327 (1912). - C. oberthüri, Fassl, Ent. Rundschau, v. 31, p. 44 (1914). - Id., 1. c., v. 33, p. 26 (1916). - C. oberthuri o., W. Rothschild, Nov. Zool., v. 23, p. 314, t. 5 , f. 3 (larva) (1916). - C. oberthüri, Fassl, Ent. Rundschau, v. 35, p. 31 (1918). - M. Hering \& W. Hopp, Deutsch. Ent. Zeit. Iris, v. 39, p. 193 (1925).

C. euphorbus (non Pavonia e. Felder), Weymer (\& Marssen), Lep. Reise Stübel, p. 33 (1890) (sec. Original in Mus. Berlin, Nr. 3248).

subsp. phokilides Fruhst. C. oberthuri p., Peru Fruhstorfer: A. Seitz, Großschmett. Erde, $\nabla .5$, p. 327 (1912). 
C. oberthurii, (part.), O. Staudinger (\& Schatz), Exot. Schmett., v. 1, p. 217 (part.) (1887). - C. oberthürii (part.), H. Stichel, Gen. Ins., Fisc. 20 et Tierreich, Lief. 25, ut antea (1901 \& 09).

- martia (God.) Morpho m., Pavonix m., (Latreille Santa

\&) J. B. Godart, Ene. Méth., v. 9, p. 450, Catharina, 807 (1824). - Pavonia m., E. Doubleday, Rio Grande List Lep. Brit. Mus., v. 1, p. 107 (1844). - do Sul Caligo m., (E. Doubleday \&) J. O. Westwood, Gen. Diurn. Lep., v. 2, p. 342 (1851). - Herrich-Schäffer, Corr.-Bl. Zool.-Min. Ver. Regensb., v. 19, p. 65 (1865). - W. F. Kirby, Cat. Diurn. Lep., p. 127 (1871). - Pavonia m., id., Cat. Coll. Diurn. Lep. Hewitson, p. 110 (1879). - Caligo m., O. Staudinger (\& Schatz), Exot. Schmett., v. 1, t. 73, p. 217 (1886 \& 87). - Röber, 1. c., จ. 2, p. 194 (1889). - Weymer, Ent. Zeit. Stettin, V. 55 (1894), p. 322 (1895). - Fruhstorfer, Doutsch. Ent. Zeit. Iris, v. 16 (1903), p. 320 (1901). - II. Stichel, Gen. Ins., Fasc. 20, p. 40 (1901). - Id., Tierreich, Lief. 25, p. 208 (1909). - Fruhstorfer: A. Seitz, Großschmett. Erde, v. 5, p. 327 , t. 65 b (1912). - Giacomelli, Rev. Chil. Hist. Nat,, v. 27, p. 16 (1921). - F. Hoffmann, Zeit. wiss. Ins.-Biol., v. 25, p. 95, 96 (ovum, not. biol.) (1930).

\section{c. Cohors Atreiformes}

H. Stichel, Gen. Ins., Fasc. 20, p. 40 (1904). - Id., Tierreich, Lief. 25, p. 210 (1909).

atreus (Koll.) Morpho a., Kollar, Denkschr. Akad. Panama,

Wiss. Wien. Mathem. Naturw. Kí., v. Venezuela

1, p. 356 (1849). - Caligo a., H. Stichel, -Peru

Gen. Ins., Fasc. 20, p. 40 (1904). - Id.,

Tierreich, Lief. 25, p. 210 (1909). - Fruhstorfer: A. Seitz, Großschmett. Ende, v. 5, p. 326 (part.) (1912).

- subsp. a treus (Kioll.) Morpho a., Kollar, ut Nord-, antea, t. 44, f. $12(1849)$. - Caligo a., W. Zentral-, F. Kirby, Cat. Diurn. Lop., p. 128 (part.) Ost(1871). - Id., Entomologist, v. 11, p. 27 Colombia (1878). - F. D. Godman \& O. Sulvin, Biol. Centr.-Amer., Lep.-Rhop., v. 1, p. 135 (part.) (1881). - O. Staudinger (\& Schatz), Exot. Schmett., v. 1, t. 75 ; p. 215 (distrib. part.) $(1886$ \& 87$)$. - L. Glaser, Cat. Etymol. Col. Lep., p. 357 (1887). - Röber: O. Staudinger (\& Schatz), Exot. Schmett., v. 2, t. 32 (Morphol.); p. 193 (1888 \& 89). - Truhstorfer, Deutsch. Ent. Zeit. Iris, v. 16 (1903), p. 318 (distr. part.) (1904). - C. a. a., H. Stichel, Gen. Ins., Fase. 20, p. 40 (part.) (1904). Id., Tierreich, Lief. 25, p. 212 (part.) (1909). - Frubstorfer: A. Seitz, Großschmett. Erde, v. 5, p. 326 (1912). - 
C. a., Fassl, Ent. Rundschau, v. 30, p. 14, 15 (1913).

C. a j a $x$, Herrich-Schäffer, Corr.-Bl. Zool.Min. Ver. Regensb., v. 19, p. 65 (part. in cit.) (1865). - Pavonia a., W. F. Kirby, Cat. Coll. Diurn. Lep. Hewitson, p. 110 (1879).

Pan'onia iris (Peale MIS), W.F. Kirby, Papilio, v. 4.p. 104 (1884). - Barnes \& Benjamin, Insecut. Inscit. Menstr., v. 15, 1926, p. 158 (1927).

subsp. dionysos Fruhst. C. a. d., Fruhstor- Panama, fer: A. Soitz, Großschmett. Erde, v. 5, p. (Chiriqui), 326 (1912). (Subsp, vix conserv.!)

$P a v o$ nia a jax (non Doubleday), H. W. Bates, Proc. Zool. Soc. London, p. 248 (1863). - Boisduval, Consid. Lép. Guatemala, p. 57 (distrib. part.) (1870).

Caligo atreus, F. D. Godman \& O. Salvin, ut antea (part. in cit.) (1881). - O. Staudinger, ut antea (part.) (1887). - Fruhstorfer, ut antea (part.) (1904). - H. Stichel, ut antea (part.) (1904 \& 1909).

subsp. a jax (Doubl.) Pavonia a., E. Doub- Venezuela leday (\& Westwood), Gen. Diurn. Lop., t. 56, f. 2 (1849); Caligo a., J. O. Westwood, v. 2, p. 342 (1851). - Caligo a., HerrichSchäffer, Corr. Bl. Zool.-Min. Ver. Regensb., v. 19 , p. 65 (sine syn.) (1865). - C. atreus ajax, H. Stichel, Gen. Ins., Fasc. 20, p. 40 (distrib. part.) (1904). - Id., Tierreich, Lief. 25, p. 213 (distrib. part.) (1909). Fruhstorfer: A. Seitz, Großschmett. Inde, v. 5, p. 326 (1912).

C. atreus (part. in cit.), W. F. Kirby, Cat. Diurn. Lep., p. 128 (1871). - F. D. Godman \& O. Salvin, Biol. Centr.-Amer, Lep.Rhop., v. 1, p. 135 (1881). - 0 . Staudinger (\& Schatz), Exot. Sehmett., v. 1, p. 215 (1887). - C. atreus, Hahnel, Deutsch. Ent. Zeit. Lep. Iris, v. 3, p. 149 (1890).

subsp. dentina Druce C. d., Herb. Druce, Peru, Trans. Ent. Soc. London, p. 155 (1871). - ?Ecuador, Id., Proc. Zool. Soc. London, p. 267 (1876). West- W. F. Kirby, Cat. Diurn. Lep., Suppl., Colombia p. 717 (1877). - F. D. Godman \& O. Salvin, Biol. Centr.-Amer., Lep.-Rhop., v. 1, p. 135 (? local form to atreus) (1881). - Iid.: Whymper, Travels Great Andes, App. Extr., p. 99 (1892). - ? C. atreus d., Fruhstorfer, Deutsch. Ent. Zeit. Iris, v. 16 (1903), p. 318 (patria?) (1904). - H. Stichel, Gen. Ins., Fase. 20, p. 40 (1904). - Id., Tierreich, Lief. 25, p. 213 , f. 43 (1909). Fruhstorfer: A. Seitz, Großschmett. Inde, v. 5, p. 326 (1912). —? C. d., Campos, Rev. Col. Nacion. Vicente Rocafuerte, Nr. 4, p. 30 (1921).

? Pavonia a jax (non Doubleday), Boisduval, Consid. Lép. Guatemala, p. 57 (part.) (1870). 
- subsp. a gesil a u s Druce C. a., Herb. Druce, Ecuador, Ann. Mag. Nat. Hist., sol: 7, v. 9, p. 321 West-

(1902). - ? C. atreus agesilarus, Fruhstorfer, Colombia Deutsch. Ent. Zeit. Iris, v. 16 (1903), p. 318 (patria?) (1904). - H. Stichel, Gen. Ins., Fasc. 20, p. 40 (1904). - Id., Tierreich, Lief. 25, p. 215 (1909). - Fruhstorfer: A. Seitz, GroBschmett. Erde, v. 5, p. 326 (1912). (Subsp. dubia, forma praeced.)

uranns (Herr.-Schäff.) Pavonia u., Caligo u., Herrich-Schäffer, Samml. außereur. Schmett., Lief. 1 , Index; t. $[1,2]$, f. 1,$2 ;$ p. 55 (1850 \& 58) (conf. Dalla Torre, Ent. Nachricht. Bl. Troppau, v. 1, 1927, p. 4, 75). C. u., (E. Doubleday \&) J. O. Westwood, Gen. Diurn. Lep., v. 2, p. 342 (1851). Herrich-Schäffer, Corr.-Bl. Zool.-Min. Ver. Regensb., v. 19 , p. 65 (1865). - G. Koch, Indo-Austral. Lep.-Fauna, ed. 1, 2, p. 116 (1865\& 73). - Pavonia u., Roisduval, Consid. Lép. Guatemala, p. 57 (1870). - Caligo u., W. F. Kirby, Cat. Diurn. Lep., p. 128 (1871). - Pavonia u., id., Cat. Coll. Diurn. Lep. Hewitson, p. 110 (1879). - Caligo u., F. D. Godman \& O. Salvin, Biol. Centr.Amer., Lep.-Rhop., v. 1, p. 136 (1881). O. Staudinger (\& Schatz), Exot. Schmett., v. 1, p. 215 (1887). - Fruhstorfer, Deutsch. Ent. Zeit. Iris, v. 16 (1903), p. 315 (1904). - H. Stichel, Gen. Ins., Frsc. 20, p. 40 (1904). - Id., Tierreich, Lief. 25, p. 216 (1909). - C. atreus u., Fruhstorfer: A. Seitz, Großschmott. Ende, v. 5, p. 326 (1912). - C. uranius (!), Gibbs, Proc. Ent. Soc. London, p. XLVT (1912).

tele machrs (How.) Pavonia t., Hewitson, Zoologist, v. 8, p. 2976 (XII. 1850).

\section{Sectio Anagraphi}

H. Stichel, Gen. Ins., Fasc. 20, p. 41 (1904). - Id., Tierreich, Lief. 25, p .217 (1909). - Fruhstorfer: A. Seitz, Großschmett. Erde, v. 5, p. 314 (1912).

H. Stichel, ut antea (1904 \& 09).

\section{a. Cohors Oileiformes}

oedipus Stich. C. o., H. Stichel, Ins.-Börse, v. 20, p. Colombia389 (1903). - Id., Gen. Ins., Fasc. 20, p. Honduras, 41 (1904). - Id., Tierroich, Lief. 25, p. PSurinam 217 (1909). - Fruhstorfer: A. Soitz, Großschmett. Erde, v. 5, p. 315 (1912).

subsp. o edipus Stich. C. o. (Pavonia o. Colombia Maassen MS), H. Stichel, ut antea (1903).

- C. o. 0., id., Gen. Ins., Fasc. '20, p. 41 (1904). - C. o., Fruhstorfer, Deutsch. Ent. Zeit. Iris, v. 16 (1903), p. $319(1904)$. - C.o. o., H. Stichel, Tierreich, Lief. 25, p. 219 (forma principalis) (1909). - Fruhstorfer: A. Seitz, Großschmett. Erde, v. 5, p. 315 (1912). 
C. teucer (non Papilio $t$. Linné), Weymer (\& Maassen), Lep. Reise Stübel, p. 9 (1890). forma noct ur na Stich. C. o. ab. nocturnus, H. Stichel, Ins.-Börso, จ. 20, p. 389 (1903). - C. o. o. forma n., id., Gen. Ins., Fase. 20, p. 41 (1904). - C. o. o. forma nocturna, id., Tierreicli, Lief. 25, p. 220 (1909). - C. o. n., Fruhstorfer: A. Seitz, Großschmett. Erde, v. 5, p. 315 (Nebenform) (1912).

subsp. Fruhstorferi Stich. C. f., H. Sti- Honduras, chel, Ins.-Börse, v. 21, p. 21 (1904). Fruhstorfer, Deutsch. Ent. Zeit. Iris, v. 16 (1903), p. 320 (1904). - C. ocdipus f., H. Stichel, Gen. Ins., Fasc. 20, p. 41 (1904). Id,, Tierreich, Lief. 25, p. 220 (1909). Fruhstorfer: A. Seitz, Großschmett. Erde, v. 5, t. $64 \mathrm{c}$, p. 315 (1911 \& 12). - Strand, Soc. Ent., v. 33, p. 19 (1918).

? Pavonia teucer (non Papilio t. Linné), Boisduval, Consıd. Lép. Guatemala, p. 56 (1870). oileus (Feld.) Pavonia o., C. \& R. Felder, Wien. Ent. Monatschr., v. 5, p. 111 (1861).

Typ.:

Colombia

(?Muzo)

Costa Rica

?Surinam

Mittel-

Amerika-

Bolivia,

? Argen-

tinien

subsp. oileus (Feld.) Pavonia o., C. \& Venezuela, R. Felder, ut antea, p. 111 (part.) (1861). H. W. Bates, Proc. Zool. Soc. Landon, p. 248 (1863). - C. \& R. Felder, Reise Novara, v. 2. IT, p. 454 (part.), t. 65, f. 2 (1866). - Caligo o., Iferrich-Schäffer, Corr.-B1. Zool.-Min. Ver. Regensb., v. 19, p. 65 (1865). - IV. F. Kirby, Cat. Diurn. Lop., p. 128 (1871). - F. D. Gorman \& O. Salvin, Biol. Centr.-Amer. Lep.-Rhop., v. 1, p. 132 (cit. part.) (1881). - O. Staudinger (\& Schatz), Exot. Schmett., v. 1, p. 216 (part.) (1887). - C. o. o., Fruhstorfer, Deutsch. Ent. Zeit. Iris, v. 16 (1903), p. 316, 319 (1904). H. Stichel, Ins.-Börse, v. 21, p. 203 (1904). - Id., Gen. Ins., Fasc. 20, p. 41 (cit. part.) (1904). - Td., Tierreich, Lief. 25, p. 223 (cit. nonnull. except.), f. 44 (1909). - ? C. o., Fassl, Faun. Exot., v. 1, p. 26 (1911). c. o. o., Fruhstorfer: A. Seitz, Großsclimett. Erde, v. 5, p. 315 (1912). - C. o., Fassl, Ent. Rundschau, v. 35, p. 31 (1918).

Pavonia scamander, Boisduval, Consid. Lép. Guatomala, p. 57 (part.) (1870).

subsp. hyposchesis Dyar C. o. h., Dyar, Panama Proc. U. S. Nat. Museum, v. 47, p. 144 (1914). (Subsp. dubia).

C. o ile u \&, F. D. Godman \& O. Salvin, Biol. Centr.-A mer., Lep.-Rhop., v. 1, p 132 (part.) (1881). subsp. scamander (Boisd.) Pavonia s., Guatemala, Boisduval, Consid. Lép., p. 57 (part.) (1870). Nicaragua, - Caligo s., W. F. Kirby, Cat. Diurn. Lep., Costa p. 646 (1871). - C. oileus s., H. Stichel, Rica, 
Ins.-Börse, v. 20, p. 42 (1904). - Id., Gen. Mexiko

Ins., Fasc. 20 , p. 42 (cit. part.) (1904). Id., Tierreich, Lief. 25, p. 224 (cit. part.) (1909). - Fruhstorfer: A. Seitz, Großschmett. Erde, v. 5, p. 315 (1912).

C. o i l e u $s$, A. G. Butler \& Herb. Druce, Proc. Zool. Soc. London, p. 338 (1874). - F. D. Godman \& O. Salvin, ut antea (part.) (18s1). - O. Staudinger (\& Schatz), Exot. Schmett., v. 1, ut antea (part.) (1887).

subsp. $p h i l a d e m u s$ Struding. C. o. var. p., West-

O. Staudinger (\& Schatz), Exot. Schmett., v. 1 , p. $216(1887)$. - C. o. p.s Fruhstorfer, Deutsch. Ent. Zeit. Tris, v. 16 (1903), p. 316, 319 (sine synon.) (1904). - Id.: A. Seitz, Großschmett. Ende, v. 5, p. 315 (1912). - ? C. p., Fassl, Ent. Rundschau, v. 35, p. 31 (1918).

C. o il evs, F. D. Godman \& O. Salvin, ut antea (part.) (18s1).

C. o. seamander, H. Stichel, Gen. Ins., Fasc. 20, p. 42 et Tierreich, Lief. 25, p. 224 (part. in cit.) (1904\& 09).

subsp. ph or $b$ a $s$ Röb. C. p., Röber, Soc. Ent., Ecuador จ. 18, p. 146 (1904). - Id., I. c., จ. 21, p. 28 (1906). - C. oileus p., H. Stichel, Soc. Ent., v. 22, p. 100 (1907). - Id., Tierreich, Lief. 25, p. 225 (1909). - Frulustorfer: A. Seitz, Großschmett. Ende, v. 5, p. 315 (1912).

Pavonia oileus, W. F. Kirby, Cat. Coll. Diurn. Lep. Hewitson, p. 110 (1879). - Caligo o., F. D. Godman \& O. Salvin, ut antea (part.) (1881). - O. Staulinger (\& Schatz), ut antea (part.) (1887). - ? Weymer (\& Maassen), Lep. Reise Stübel, p. 62 (sine cit.) (1890). - ? F. D. Godman \& O. Salvin: Whymper, Travels Great Andes, App., Extr., p. 99 (1892). - ? C. oileos (!), Campos, Rev. Col. Nacion. Vicente Rocafuerte, Nr. 4, p. 30 (1921).

C. s camander, Dogniu, Lép. Loja, p. 34 (1891). - C. oileus s., H. Stichel, Gen. Ins., Fasc. 20 , p. 42 (in cit.); Fasc. 31 , p. 16 , Addenda syn. C. phorbas (1904\& 05).

C. o. philademus, Fruhstorfer, Deutsch. Ent. Zeit. Iris, v. 16 (1903), p. 319 (in cit.) (1904).

C. prometheus epimetheus (non Pavonia e. Felder), H. Stichel, Gen. Ins., Fasc. 20 , p. 30 (lapsus, corr. Fasc. 31 , p. 16) (1904 \& 05).

subsp. umbratilis Stich. C. o. u., II. Sti- Peru chel, Ins.-Börse, v. 20, p. 389 (1903). - Id., I. c., v. 21, p. 204 (1904). - Id., Gen. Ins., Fasc. 20, p. 41 (1904). - Fruhstorfer;, Deutsch. Ent. Zeit. Iris, v. 16 (1903), p. 316, 319 (1904). - H. Stichel, Tierreich, Lief. 25, p. 225 (1909). - Fruhstorfer: A. Seitz, Großschmett. Erde, v. 5, p. 316 (1912).

Colombia

(Antioquia) 
C. oileus (part.), C. \& R. Felder, Wien. Ent. Monatschr., v. 5, p. 111 (1861). - O. Staudinger (\& Schatz), Exot. Schmett., v. 1 , p. 216 (1887).

- subsp. $p h i l i n o s$ Fruhst. C. o. p., Fruhstor- Bolivia, fer, Ins.-Börse, v. 20 , p. 413 (patria vera?) ? Peru (1903). - C. p. o. (philoxenus Stauding. i. 1.), id., Deutsch. Ent. Zeit. Iris, v. 16 (1903), p. 316, 317 (patria vera ?) (1904). - H. Stichel, Ins.-Börse, v. 21, p. 204 (1904). Id., Gen. Ins., Fasc. 20, p. 42 (1904). Id., Tierreich, Lief. 25, p. 225 (1909). Fruhstorfer: A. Seitz, Großschmett. Erde, v. 5, p. 316, t. 65 a (1912). - ? C. philinus (!), P. Köhler, Fauna Argent., pars 1 (Zeit. wiss. Ins.-Biol., v. 18, Beiheft), p. 22 (1923).

- zeuxipjus Druce C. z., Herb. Druce, Ann. Mag. Ecuador Nat. Hist., ser. 7, v. 9, p. 321 (1902). Fruhstorfer, Deutsch. Ent. Zoit. Iris, $\checkmark$. 16 (1903), p. 319 (1904). - H. Stichel, Gen. Ins., Fasc. 20, p. 42 (1904). - Id., Tierreich, Lief. 25 , p. 226 , f. 45 (1909). Fruhstorfer: A. Seitz, Großschmett. Erde, v. 5 , p. 316 , t. $65 \mathrm{~b}$ (1912).

C. o il cus (non Pavonia o. Felder), Weymer (\& Maassen), Lep. Reise Stübel, p. 62 (1890). placidinnus Stauding. C. p., O. Staudinger (\& Schatz), West-

Exot. Schmett., v. 1, p. 216 (1887). - O. Amazonas, Michael, Deutsch. Ent. Zeit. Lep. (Tris), v. Ecuador 7, p. 232 (1895). - Fruhstorfer, Deutsch. Ent. Zeit. Iris, v. 16 (1903), p. 319 (1904). - H. Stichel, Gen. Ins., Fasc. 20, p. 42 (1904). - Id., Tierreich, Lief. 25, p. 228 f. 46 (ㅇ) (1909). - Fruhstorfer: A. Seitz, Großschmett. Ende, v. 5, p. 228 (1912).

forma m ic ans Röb. C. p. var. m., Röber, Typ.:

Soc. Ent., v. 18, p. 146 (1904). - Id., 1. c., Ecuador v. 21 , p. $28(1906)$. - C. p. m., Fruhstorfer, Ent. Zeit. Stettin, v. 6S, p. 133 (1907). Id., A. Seitz, Großschmett. Erde, v. 5, p. 316 (1912). (Nóm. vix conserv.)

C. placidianus (part. in eit.), H. Stichel, Gen. Ins., Fasc. 20 et Tiemeich, Lief. 25 , ut antea (1904 \& 09).

\section{b. Cohors Belfraoformes}

H. Stichel, Gen. Ins., Fasc. 20, p. 42 (1904). - Id., Tierreich, Lief. 25, p. 231 (1909).

- Beltrao (IIl.) Papilio (Heros) b., Illiger, Mag. Mittel- und Insektenk., v. 1, p. 199 (1801). - Caligo b., Süd(Jac. Hübner \&) C. Geyer, Samml. Exot. Brasilien Schmett., v. 3 , t. 15 ; t. 15 . IT $(1826-41)$. (E. Doubleday \&) J. O. Westwood, Gen. Diurn. Lep., v. 2, p. 342 (1851). - Herrich-Schäffer, Corr.-Bl. Zool.-Min. Ver. Regensb., v. 19, p. 65 (1865). - G. Koch, IndoAustral. Lep.-Fauna, ed. 1, 2, p. $116(1865$ \& 73). - W. F. Kirby, Cat. Diurn. Lep.,

(Espirito

Santo

bis Rio

Grande do

Sul),

Nond-

Argentinien 
p. 128; Suppl., p. 717 (1871 \& 77). - Id., Entomologist, v. 11, p. 27 (1878). - G. W. Müller, Zool. Jahrb. Syst., v. 1, p. 597 (Biol.) (1886). - O. Staudinger (\& Schatz), Exot. Schmett., v. 1, t. 75 (1886). - Glaser, Cat. Istymol. Col. Iep., p. 358 (1887). Röber: O. Staudinger \& Schatz, Exot. Schmett., $\nabla .2$, p. $19 \pm$ (1889). - W. Reuter, Acta Soc. Sci. Fenn., v. 22, p. 113 (palpus) (1896). - W. F. Kirby, Samml. Exot. Schmett. Hübner \& Geyer, Neue Ausg., v. 3 , t. $453(15), 454(16)(1902)$. - H. Sticliel, Gen. Ins., Fasc. 20, p. 42 (1904). Silva, Relat. Contrib. Hist. Nat. Lep. Brasil (Congr. Scient. Lat.-Amer., 1905, v. 3B), p. 92 , t. 17 , f. 58 (1907). - H. Stichel, Tierreich, Lief. 25, p. 231 (1909). - Fruhstorfer: A. Seitz, GroBschmett. Erde, $\nabla$. 5, p. 316, t. 65 b (1912). - Forreira d'Almeida, Mélang. Lépid., p. 108 (Biol.) (1922). - Zikán, Ent. Rundschau, v. 45, p. 11 (1928). - A. Seitz, l. c., p. 31 (ovum) (1928). - F. IIoffmann, Zeit. wiss. Ins.Biol., v. 25, p. 95,96 (not. biol.) (1930). demosthenes (Perry) Papilio d., Perry, Arcana, v. 2, t. 31 (1811). - Caligo d., (1:. Doubleday \&) J. O. Wostwood, Gen. Diurn. Iep., v. 2, p. 342 (1851). - Herrich-Schäffer, Corr.-Bl. Zool.-Min. Ter, Regensb., v. 19 , p. 65 (1865). - IV. F. Kirby, Cat. Diurn. Lep., p. 128 (1871). - L. Glaser, Cat. Etymol. Col. Lep., p. 366 (1887). Bönninghausen, Vorh. Ver. Naturw. Unterh. Hamburg, v. 9 (1894-95), p. 37 (1896). O. Staudinger (\& Schatz), Exot. Schmett., v. 1, p. 215 (1887). - W. F. Kirby, Samml. Exot. Sclimett. Hübner \& Geyer, Neue Ausg., v. 3, p. 53 (1901). - Fruhstorfer, Deutsch. Ent. Zeit. Iris, v. 16 (1903), p. 318 (not. biol.) (1904).

Reinwardtianu\& (Drap.) Morpho r.,

Drapier, Ann. Gén. Sci. Phys. Bruxelles, v. 7 , p. 278 , t. $109(1820)$.

in achis (God.) Morpho i., Pavonia i., (Iatreille \&) J. B. Godart, Ene. Méth., v. 9, p. 449, 807 (1824). - M. i., H. Lucas, Hist. Lép. Exot., p. 138, t. 74 (1835). - Pavonia i., I. Doubleday, List Lep. Brit. Mus., v. 1, p. 107 (1844). - Caligo i., (E. Doubloday \&) J. O. Westwood, Gen. Diurn. Lep., v. 2, p. 342 (1851). - Ménétriés, Enum. Corp. Anim. Ac. Petropol., Lep., pars 1, p. 38 (1855). - Carus-Gerstäckel, IIandb. Zool., v. 2, p. 231 (1863). - Prittwitz, Ent. Zeit. Stettin, v. 26, p. 309 (not. biol.) (1865). Herrich-Schäffer, Corr:-Bl. Zool.-Min. Ver. Regensb., v. 19, p. 65 (1865). - Burmeister, Rev. Mag. Zool., ser. 3, v. 1, p. 31, t. 1, f. 1 (larvae caput); t. 5 (Metamorph.) (1873). 
- W. F. Kirby, Cat. Diurn. Lep., Suppl., p. 717 (1877). - Schmarda, Zool., ed. 2, v. 2, p. 158 (1878). - Burmeister, Descr. Phys. Argent., v. 5. II, p. 20, t. 16 (1879). - Pavonia i., W. F. Kir.by, Cat. Coll. Diurn. 20

Lep. Hewitson, p. 110 (1879).

12 genera

81 specics

\section{Addenda et Corrigenda}

Pag. 1, lin. $1 \mathrm{ab}$ imo adde: „Brassolinae, (Dukinfield Jones \&) F. Moore, Proc. Liter. Philos. Soc. Liverpool, sessio 71, Nr. 36, p. 331, 337 (1882).“

Pag. 14, subsp. cyllastros, lin. 9 ab imo pro, ,t. 62 d-1911, 12 )" lege: t. 62 d (cyllastrus, $0^{7}$, \&); p. 328, corr. p. 1140 (1911, $12 \& 24)$.

Pag. 15, eyllarus, lin. 12 ab imo adde "Fruhstorfer: A. Seitz, Großschmett. Evide, v. 5, p. 328, t. 62 d (1912)“.

Pag. 17, Caligonilli, adde: „Brassolidi (part.), E. Reuter, Acta Soc. Sci. Fenn., v. 22, p. 553 (1896).

Pag. 23, lin. 9 addo "Dassiophthalma (!), G. Koch, Indo-Austral, Lep.-Tauna, ed. 2, p. 116 (1873)."

Pag. 25, subsp. creus a, lin. 25 addc; ,D. creusa, E. Reuter, Acta Soc. Sci. Fenn., v. 22, p. $113(1896)^{\prime \prime}$.

Pag. 33, subsp. c a s sia e, lin. 34 adde: O.c., Dcwitz, Arch. Naturg., v. 44, p. 6 (Metam.) (1878)".

Pag. 35, O. gl y c erie, lin. 36 adde; „? Distant, Proc. Ent. Soc. London, p. XIII (1876) (vide quoque 0 . quiteria quirinus)".

Pag. 45, subsp. cuspidatu 8, adde: „O. invirae (non Pot. $i$. Hübner), A. G. Butler \& Herb. Druce, Proc. Zool. Soc. London, p. 339 (cit. part.) (1881).

Pag. 47, subsp. Me riana e, lin. 16 adde: "? - Merian, Ins. Surinam, t. 8 (larva solum, non imago: = Peridromia amphinome L.) (1705) (conf. Burmeister, Abh. Naturf. Ges. Halle, v. 2, 185t, Sitzgsber. p. 61: Pavonia cassiae aut $P$. xanthus) (vide quoque J. O. Westwood, Trans. Ent. Soc. London, 1856, n. ser., v. 4, p. 115: Opsiphanes cassiae) (non Pap. c. L.) “"

Pag. 47, O. cas s i a e, lin. 24 ab imo, adde: „F. D. Godman \& O. Salvin, Biol. Centr.-Amer., Lep.-Rhop., v. 1, p. 127 (part. in cit.) (1881)".

Pag. 49, subsp. $n u m$ a ti $u s$, addo: O. cassice (non Pap. c. L.), F. D. Godman \& O. Salvin, Biol. Centr.-Amer., Lep.-Rhop., v. 1, p. 127 (part.) (1881).

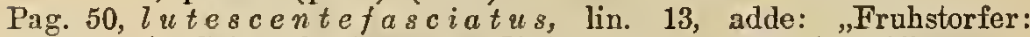
A. Seitz, Großschmett. Erde, v. 5, p. 310 (1912)".

Pag. 50, Opsiphanes, lin. 8 del. ,pars 2, p. 38 (1857)".

Pag. 51, lin. 4 adde: „E. Reuter, Acta Soc. Sci. Fenn., v. 22, p. 112 (part.) (1896)."

Pag. 56, subsp. $x$ a $n t h$ us, al. 17 ab imo adde: „? - Merian, Ins. Surinam etc, ut antea pag. 47.

Pag. 62, subsp. berceynthia, lin. 6, ab imo adde: „O. berecynthus, J. O. Westwood, Trans. Ent. Soc. London, n. ser., v. 4, p. 165 (larva sec. Stoll) (1857)."

Pag. 68, lin. 3 ab imo adde „L. Glaser, Cat. Etymol. Col. Lep., p.288 (1887).“

Pa,g. 69, Moera, lin. 14, adde: „S. H. Scudder, Hist. Sketch: Proc. Amer. Ac. So., v. 10, p. 220 (nom. praeocc.) (1875)“. 


\section{Index.}

Synonyme und ungiltige Namen sind kursiv gedruckt. - Namen, die sich auf derselben Seite wiederholen, sind nur einmal aufgeführt.

\section{Familia, Tribus, Genera, Sectiones, Cohorfes.}

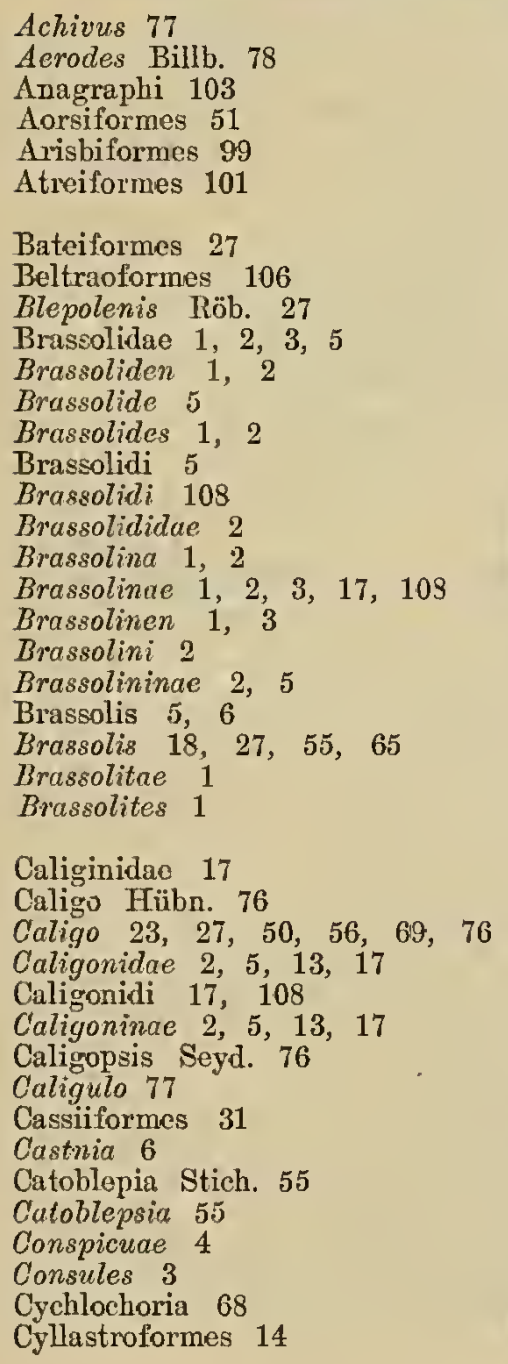

Achivus 77

Acrodes Billb. 78

Anagraphi 103

Aorsiformes 51

Arisbiformes 99

Atreiformes 101

Bateiformes 27

Beltraoformes 106

Blepolenis Röb. 27

Brassolidae 1, 2, 3, 5

Brassoliden 1, 2

Brassolide 5

Brassolides 1, 2

Brassolidi 5

Brassolidi 108

Brassolididae 2

Brassolina 1, 2

Brassolinae 1, 2, 3, 17, 103

Brassolinen 1, 3

Brassolini 2

Brassolininae 2, 5

Brassolis 5, 6

Brassoli: 18, 27, 55, 65

Brassolitae 1

Brassolites 1

Caliginidae 17

Caligo Hüibn. 76

Caligo $23,27,50,56,69,76$

Caligonidae 2, 5, 13, 17

Caligonidi 17, 108

Caligoninae 2, 5, 13, 17

Caligopsis Seyd. 76

Caligulo 77

Cassiiformes 31

Castria 6

Catoblepia Stich. 55

Catoblepsia 55

Conspicuae 4

Consules 3

Cychlochoria 68

Cyllastroformes 14

Danai festiui 3

Danai Festivi 3

Danai festivi 3

Danaus 6, 18, 27, 55

Danaus (Festivres) 6,18, 27, 55

Dasiophtalma 23

Dassiophthalma 108 (addenda)

Dasyophthalma Westw. 23

Dasyopthalma 23

Desmidocosmeti 51

Dinastor 17

Dynaror 22

Dynastor Doubl. 17, 18

Dyophthalmi 56

Eques 69, 77, 78

Eques (Achivus) 69, 78

Equites 3

Equites Achiui 3

Equites Archiui 3

Equites Archivi 3

Equites Troes 3

Eryphane 68

Eryphanes 68, 69,

Eryphanis Boisd. 68

Eryphanis 51, 76, 79

Festivi 3

Graphiophori 79

IIamadryas 78

Heros Illig. 78

Megastes Boisd. 6, 18

Meropis Boisd. 26, 31

Moera IIübn. 69, 108

Morphida 4

Morphidae 1, 4

Morphina 4

Morphinae 4

Morphitae 1, 4

Morphites 1, 4 
Morpho 18, 23, 27, 50, 55, 65, $\mid$ Papiliones Nymphales 4

78

Morphoides 4

Morphonides 4

Narope Doubl. 13, 14

Naropidi 13

Naropina Röb. 14, 17

Nephrocoria 66

Nesopiformes 16

Nymphales 3

Nympholes Gemmati 3

Nymphales gemmati 3

Nymphalidae 3, 4

Nymphaliden 4

Nymphalides 3

Nymphalididae 4

Nympholiens 3, 4

Nymphalinue 4

Nymphalis 6, 65

N ymplatis Gemmati 3

Nymphatis (Gemmatus) 27, 65

Oileiformes 103

Opoptera Auriv. 50

Opsifanes 26

Opsiplanes Doubl. 26

Opsiphanes $23,50,51,55,56$, $65,69,78$

Papilionides 4

Papilionina 4
Pavonia 18, 23, 27, 50, 55, 65, $69,76,78$

Pavonie 50, 78

Pavonidoe 2

Pavonides 1, 5

Penetes Doubl. 12, 13

Peracurii 17

Peragnosti 55

Perlota 4

Pilopoceili 14

Potamides Conspicuae 4

Potamides Superbae 4

Potamis Hübn. 23, 69

Potamis Conspicua 78

Potomis conspicua Hübn. 78

Potamis Superba 18, 55

Potamis superba Hübn. 18, 23 , 27,55

Psilocraspeda 69

Satyridae 5

Satyre 6, 78

Satyrinae 5

Satyromorpha 5

Satyromorphen 5

Satyromorphi 5

Selenophanes Staudgr. 65

Symiformes 53

Teucriformes 79

Tricothamnodes 73

\section{Species, Subspecies, Formae.}

acadina God. 54

adjecta Stich. 64

odjecta 65

aequatorialis Sticl. 49

aesacus Herr.-Schäff. 73

aethon 63

agamemnon Weym. 98

ogamemnon 98

agasthenes Fruhst. 45, 46

agesilaus Druce 102

ajax Doubl. 102

ojax 102

albopunctum Stich. 17

alcimedon Dalm. 54

amphimedon Feld. 71

amplimedon 71

amphinome L. 108

amphirhoe Hübn. 59

amphirhoe ? 57,59

amphitoe 60

amplificatus Stich. 46, 47

amplificatus 46

amplior Stich 67 anartes Hew. 15, 16

anartes 15,16

anoxandra God. 25

andromeda Stich. 67

androsthenes 45

Anaxareta 20

Anaxarete 19

anaxareto Cr. 19,20

anascarete 21

anaxarethus 20

anaximandrus Fruhst. 87

andicolens Stich. 91

angusta Strand 64

aorsa God. 51

apollo 97

apollonidas Fruhst. 97

ardens Stich. 9

ariphron Fruhst. 97

arisbe Hübn. 99

arisbe 100

aristophanes Fruhst. 99

arsippe Hopff. 52

aspherus Fruhst. 28 
astyalus Burm. 11

astyra God. 10, 11

Atlas, Papilio Seba 95

atlas Röb. 86

atlas 86

atreus Koll. 101

atreus 102

augeias Fruhst. 40,41

Aurivillii Röb. 39

automedaena Hübn. 71

automedon Cram. et al. 70, 71,

72,75

automedon var. 74

badius Stich. 42,43

badius 43

baronesa Stich. 25

bassus Feld. 31

batea Hübn. 25

batea 29

beata Fruhst. 29

beata? 30

belisar Stich. 61

belleropbon Stich. 89

beltrao IIl. 106

Berchmansi Stich. 31

Berecynthia 62

berecynthia $\mathrm{Cr} .62,63,108$

berecynthia $61,62,65$

berecynthina Hopff. 64

berecynthius 62

berecynthus 62, 63, 65, 108

berecyntia 65

berecyntius 63

bogotanus Dist. 38, 39

Boisduvalii Doubl. 31, 32

boliviana Rothsch. 12

bolivianus Stich. 42

bracteolata Stich. 52

brasiliensis Feld. 92

brasiliensis 93

brasiliensis ? 91

brasiliensis ab. 93

brensa 25

bubocula 74

buboculus Butl. 74

buenavista Rothsch. 65

cachi Joic. \& K. 88,89

caesia Stich. 91, 94

caesius

camena Staudgr. 43

caryatis God. 67

Cassiae 32,48

cassiae I. $32,33,108$

cassiae $34,35,36,40,47,48$, 49

cassiae ? $42,48,108$

cassiae var. 49 cassiculus Stich. 34

cassid 32

cassina Feld. 47

cassina 49

cassione 66

cassiope Cram. 66

cassiopeia Stauding. 67

cassiopeia 67

cassiopus 66

castaneus Stich. 36

catharinae Stich. 30

catharinae? 29

catherinae 30

cauca Röb. 43

ceutcria 39

cheiremon Fruhst. 72

cherocles Fruhst. 37

chiriquensis Stich. 49

colombiana Rothsch. 52

colombicola Rothsch. 54

corrosus Stich. 37, 38

costaricensis Strand 73

Crameri Feld. 34

Crameri 36

Crameri ? 49

creusa Hübn. 24, 25, 109

creusa 26

cuspidatus Stich. 45, 108

cuspidatus 45

cyllabarus Westw. 16

cyllarus Westw. 15, 108

cyllastros Doubl. 14, 108

cyllastros 15

cyllastrus 14, 108

cyllene Feld. 14, 15

cyparissa Fruhst. 61

cyparissa ? 60

dardanus Boisd. et al. 86, 87

darius F. 18,19

darius 20,21

decentius Fruhst. 34, 35

delanira Hew. 24

delcetans Joic. \& K. 91

demosthenes Perry 107

denticulata Talb. 15

denticulatus 15

dentina Druce 102

didymaon Feld. 29

didymaon 29, 30

diluta Stich. 88

dimidiata Fruhst. 11

dionysos Fruhst. 102

disyllus Fruhst. 16

ditatus Thieme 67

Dohrni Stich. 58

Donckieri Joic. \& Talb. 24

Dondoni Fassl 76

dubia Röb. 30 
excisus Rothsch. 68

excultus Stich. 68

ecuadora Joic. \& K. 82

epimetheus Feld. $86 \quad 7$

epimetheus 87, 105

crebus Röb. 42

euchlaena Fruhst. 30

euphorbus Feld. 98

euphorbus 100

euriloche Hübn. 90

eurilochus Cram. 90, 95

curilochus 84, 91, 92, 93

eurylaechus 92

eurylochus 90, 92, 93, 94

eurylochus-brasiliensis Zik. 92

eurylochus var. 91

Fabricii Boisd. 49

Fabricii 47,48

Fabricii? 50

faenius Frulst. 20

farrago Stich. 44

Flemmingi Rothsch. 59

Fruhstorferi Röb. (Opsiphan.) 53

Fruhstorferi Stich. (Caligo) 104

fulgens Rothsch. 100

fumosa Stich. 54

fuscata Sitich. 52

gainas Fruhst. 30

gaivas 30

galba Deyr. 93

galba 93

gehrardi 74

genelosa Stich. 65

geraensis Reb. 24

Gerhardi Weeks 74

glaukias Fruhst. 28

glycerie Fabr. 33, 34

glycerie 35, 36, 108

glycerie? 41

granadensis Stich. 12

Haenschi Stich. (Brassolis) 10

haenschi 12

Hänschi Röb. (Caligo) 86

hannibal Oberth. 22

hemichroa Butl. 75

hilara Stich. 52

hippolocluus Fruhst. 97

hycerie 33,34

hyposchesis Dyar 104

iapetus 82

ibycus Fruhst. 97

icterica Stich. 19

ictericus 19

idomenea Hübn. 95

idomeneus L. 94 idomencus 81, 83, 91, 97, 98, 99

idomenides Fruhst. 97

idomeus 95

idomineus 96

ilioneus 79, 83, 84, 85, 92

illionea 83,85

illioneus $\mathrm{Or}$. 81, 82, 95

inachis God. 107

incolumis Stich. 39

insulana Stich. 81

insulanus 81, 94

intcrmedia 45

intermodius Stich. 45

intermedius 45

invirae Hübn. 44

invirae 39, 45, 47, 61, 108

invirae? 45,46

isagoras Fruhst. 46

isthmia Bat. 11, 12

japetus Stich. 82

jomeneus 96

joasa Joic. \& $K .81$

josephus Goxlm. \& Salv. 68

juruanus Fruhst. 74

kleisthenes Fruhst. 37

Langsdorfi Mén. 11

latifascia Rothsch. 39

latitaenia Fruhst. 63

Laurentii Stich. 9

ledon Fruhst. 45

litura Fruhst. 52

livius Stauding. 91

livius 92,94

livoris Stauding. 88

lucullus Fruhst. 35

lurida Stich. 9

luteipennis Butl. 31

lutescentefasciatus 50, 108

lutescente-fasciatus 50

luxuriosa Stich. 64

luxuriosus 64

lycaon Lucas 24

lycomedon Feld. 72

lycomedon 73

macasana Strand 64

macnosiris Doubl. 21

maculata Niep. 22

maculatus 22

magnalis Stich. 59

mardonius Fruhst. 20

mardonus 20

maritima Stich. 10

marmorata Schaus 16

mars 97

marsus Stich. 97 
martia God. 101

memnou Feld. 87

memnon 85, 87, 89, 100

menes Fruhst. 88

menes 88,89

menus 88

menoetius Standing. 99

menoetius 98

Merianae Stich. 32, 47, 49

meridionalis Staudgr. 42

meridionalis $42,46,47$

mesomerista stich. 37

micans Röb. 106

midas Stich. 61

minor Kaye 94

morpbeus Stich. 93

mutatus Stich. 43, 44

mylasa Fruhst. 41

napoleo 22

Napoleon Doubl. 22

nesope Hew. 16

nicandrus Frulst. 44

nidas 97

nocturna Stich. 102

notanda 49

notandus Stich. 49

novicia Stich. 72

nubila Fruhst. 81

nubilus 81

numatius Fruhst. 49, 108

obidona Fruhst. (Caligo) 81

oberon Butl. 84

oberon $\$ 4$

oberthuri 100

oberthuri: 100,101

oberthiori 100

oberthurii 101

Oberthiirii Deyr. 100

obidonius 40

obidonus Fruhst. (Opsiphan.) 40

obidonus (Caligo) 81

oculata Stich. 31

oedipus Stich. 103

oethon Fabr. 63

oileus Feld. 104

oileus 104, 105, 106

opimus Stauding. 75

opimus 75

oresbios Fruhst. 42

orgetorix Hew. 58, 59

orgetory $x$ 58, 59

ornamentalis Stich. 12

pallida Fruhst. 92

pallidus 92

pampeiro Fruhst. 85

pamphanis Doubl. 13

Lepidopterorum Catalogus 51 . panniculus Stich. 16, 17

panormus Röb. 29

pavo Röb. 88,89

pavonides Fruhst. 89

pelcus Stich. 88

periphetes Fruhst. 49

periphetes trans. 47

peruanus Rothsch. 39

phamacer 22

phamaces Stich. 22

pheidriades Fruhst. 85

philademus Stauding. 105

philademus 105

philinos Fruhst. 106

philinus 106

philocala Stich. 11

philomela Stich. 9

philon Fruhst. 42

philoxenus Stauding. 106

phokilides Fruhst. 100

phorbas Röb. 105

phorbas 86,87

phorkys Fruhst. 82

phoroueus Fruhst. 82

phrataphernes Fruhst. 39

phryasus Fruhst. 94

phylas Fruhst. 40

piccata Stich. 17

placentia Fruhst. 67

placidianus Stauding. 106

placidianus 106

placita Stich. 60

polyxena Meerb. (Eryphan.) 69, 70

polyxena 71

polyrenus Stich. (Caligo) 84

polyxenus 84

populus Röb. 20

praecana Stich. 97

praegrandis Fruhst. 30

prataphernes 39

praxsiodus Fruhst. 85

principesa Stich. 24

privata Fruhst. 93

prometheus Koll. 85

prometheus 87,88

pseudocassiae Fruhst. 34

pseudophilon Fruhst. 46

pseudospadis Strand 38

pudicus Fruhst. 34

pusilla Röb. 17

pusillus Stich. 74, 75

quaestor Stich. 41

quaestor 41

quinteria 42

quirinalis Staudgr. 41

quirinus Godm. \& Salv. 41, 108 
quirinus 41

quirinus ? 42

Quiteria 39

quiteria Stoll 39,40

quiteria 34, 40,41, 42, 57

quiteria ? 40,41

quiterie 40

quiterius 39

rectifasciata Fruhst. 46 reevesi 75

Reovesii Doubl. 74

Reinwarltinnus Drap. 107

relucens Fruhst. 45

remoliata 46

remoliatus Fruhst. 42, 46

remuliata 46

reveesi 75

revesii 74

rhootus Stauding. 96

rivalis Niep. 58

rivesii 74

rubigatus Stich. 35

rufescens Rothsch. 10

rufescente-fuscus Groze 9

rusina God. 23, 24

Sallei Doubl. 43

saltus Kaye 84

Saphorac 8

sarastro Staudgr. 16

saronia God. 28

scrmandor Boisd. 104

scamander 104, 105

selectus Staudgr. 58

seleucida Hew. 76

semicarulca Joic. \& K. 82

sikvon Fruhst. 37

singularis WVeym. 60

sodalis Röb. 53

sophora 8

Sophorae 7

sophorae I. $6,7,8,9$

sophorcte $9,10,25$

sophord 7

soranus IVestw. 58

soranus? 58

sosigenes Fruhst. 61

sosius Staudgr. 53

spadix Stich. 38

spintasus Fruhst. 60

spintharus Fruhst. 72,73

Staudingeri Godm. \& Salv. 55

Sticheli Röb. 45

stratonides Fruhst. 99

strix Bat. 21

strix 22

strophios Fruhst. 35

strophios ? 38 superba Stauding. 98

superbus 98

stygianus Bti. 20, 21

stygius Staudgr. 15

subsericea 29,30

subsericeus Funbt. 29

sulana Fruhst. 94

sulanus 94

sulcius Staudgr. 53

sulcius 53

superba Stauding. 97

superbus 97

supremus Stich. 67

sutor Stich. 17

suzanna Deyr. 81

suzanna 82

syllabus Staudgr. 15

syme Hiibn. 53, 54

syme 54

taenius 20

tamarinde 36

tamarindi Feld. 36, 37

tamarindi 37,38

tamarindi ? 38

taramela God. 87, 100

telemachus Hew. 103

telamonius Feld. 88

telamonitus $82,87,88,89$

toncer 80

tenuifasciata Neust. 68

terenzius Fruhst. 35, 37, 38

testacea Godm. \& Salv. 15

teucer I. 79

teucer 82, 83, 84, 87, 90, 91, 196, 103, 104

teuces 79

teucra 80

theogenis Fruhst. 67

tristis Stauding. 72

umbratilis Stich. 105

unditaenia Fruhst. 65

uranius 103

uranus Herr.-Schäff. 103

velata Stich. 63

velatus 63

vercingetoryx Staudgr. 64

versitincta Stich. 58

vertebralis Butl. 26

vicenciona Fruhst. 64

vivalis 58

vulpeculus Stich. 9, 10

wardi 71

Wardii Boisd. 71

Wardii 73

Wilhelminae Röb. 30 
xanthicles Godm. \& Salv. 60 xanthis 57

xanthus L. 56, 57, 108

xanthus 9, 34, 40, 58, 59, 60, 61 xanthus ? $58,60,61,108$

xiphios 37

xiphos Fruhst. 37 zelotes Hew. 36 zelus Stich. 36 zelys 36

zeuxippus Druce 106

zolvizora Hew. 75

zuzanna 82 



\section{W. J U N K, Verlag und Antiquariat für Entomologie Berlin W. 15}

Pars 35, 37, 39: F. Bryk, Papilionidate. 1929-1930. 675 p.

36: II. Neustetter, Heliconiidae. 1929. 136 p.

38, 40, 41, 44: H. Stichel, Riodinidae 1930-1931. 795 p.

42: F. Bryk, Dioptidac. 1930. 65 p.

43, 46, 48: М. (4aede, Sutylidae. 1931. 759 p.

45: F. Bryk, Pericopinac. 1931. 57 p.

47: H. II. Sheparl, Herperidae: Subfam. Psoginac I. 1931, 144 p.

49: M. Gaede, Drepanidace 1931. $60 \mathrm{p}$.

50: II. Gaede, Jimallonidac. 1931. 21 p.

Im Druck:

52: M. Gaede, Aganaidae.

In Vorbereitung:

F. Bryk, Lymantriidae, Zygaenidae (excl. Zygaena speeies palaearet.).

A. Busck, Stenomiclac.

A. Corti, Agrotinae palacareticac.

J. Draescke, Isyenenidne.

M. Gaede, Notodontidac, „Tincoidea":
1. Incurvariiklte
5. Opostegidae
9. Momphidate
2. Tischeriidac
6. Tineidae
10. Elachistidae
3. Heliozelidae
7. Gelechiidate
11. Cosmopterygidae
4. Nepticulidae
8. Colcophoridae
12. Lyonetiidate
13. Cemiostomidae.

J. Kíremky, Dauaididae, Pyralididae.

I. 13. Pront, Sterrhinae.

II. Schuissler, Satnmiidae, Enpterotidae.

II. II. Shepard, Hesperiidac II et III.

II. Stichel, Discophoridae, Amathisiidae, Iyantidae, Nymphalinae, A paturinae.

E. Strand, Noctuidae.

G. Talbot, Pierididae.

Druck von Gustav Feller, Neubrandenburg. 